\title{
Deoxofluorination of Aliphatic Carboxylic Acids: a Route to Trifluoromethyl-Substituted Derivatives
}

\author{
Maksym Bugera, ${ }^{\dagger, \dagger \dagger}$ Serhii Trofymchuk, ${ }^{\dagger, \ddagger}$ Karen Tarasenko, ${ }^{\dagger \dagger}$ Olga Zaporozhets, ${ }^{\S}$ Yurii \\ Pustovit, ${ }^{\dagger \dagger}$ and Pavel K. Mykhailiuk ${ }^{\dagger, \S_{*}}$ \\ †Enamine Ltd.; Chervonotkatska 78, 02094 Kyiv (Ukraine), www.enamine.net; www.mykhailiukchem.org, \\ pavel.mykhailiuk@gmail.com \\ ${ }^{\dagger}$ Institute of Bioorganic Chemistry and Petrochemistry NAS of Ukraine; Murmanskaya 5, 02094 Kyiv (Ukraine) \\ Institute of Organic Chemistry NAS of Ukraine, Murmanskaya 1, 02094 Kyiv (Ukraine) \\ ${ }^{\S}$ Taras Shevchenko National University of Kyiv; Chemistry Department; Volodymyrska 64, 01601 Kyiv (Ukraine).
}


Photos.

Compound 2a . S7

Compound $3 \mathrm{a}$. $\mathrm{S} 10$

Compound $4 \mathrm{a}$. $\mathrm{S} 13$

Compound $5 \mathrm{a}$. S16

Compound $6 \mathrm{a}$ S19

Compound $7 \mathrm{a}$ S22

Compound 8a S25

Compound 9a S28

Compound 10a S31

Compound 11a S34

Compound $12 \mathrm{a}$ S37

Compound 13a $\mathrm{S} 40$

Compound 14a $\mathrm{S} 43$

Compound 15a S46

Compound $16 \mathrm{a}$ S49

Compound 17a S52

Compound $18 \mathrm{a}$ S55

Compound 19a S58

Compound 20a S61

Compound 21a S64

Compound 22a S67

Compound 23a $\mathrm{S} 70$

Compound 24a S73

Compound 25a S76

Compound 26a S79

Compound 27a S82

Compound 28a S85

Compound 29a S88

Compound 30a S91

Compound 31a S94

Compound 32a S97

Compound 33a S100

Compound 34a S103

Compound 35a S106

Compound $36 \mathrm{a}$ S109

Compound $37 \mathrm{a}$ 
Compound 38a S115

Compound 39a S117

Compound 40a S120

Compound 41a S123

Compound 42a S126

Compound 43a S129

Compound $2 \mathrm{~b}$ S132

Compound $6 \mathrm{~b}$ S135

Compound $7 \mathrm{~b}$ S138

Compound $8 \mathrm{~b}$ S141

Compound $9 \mathrm{~b}$ S144

Compound $10 \mathrm{~b}$ S147

Compound $11 \mathrm{~b}$ S150

Compound 21b S153

Compound 29b S157

Compound $30 \mathrm{~b}$ S160

Compound $31 \mathrm{~b}$. S163

Compound 39b S166

Compound 40b S169

Determination of the optical purity of compound (S)-21b S172

Crystallographic data (X-Ray) S174 


\section{Graphical reaction set-up and work-up}

Synthesis of 39a

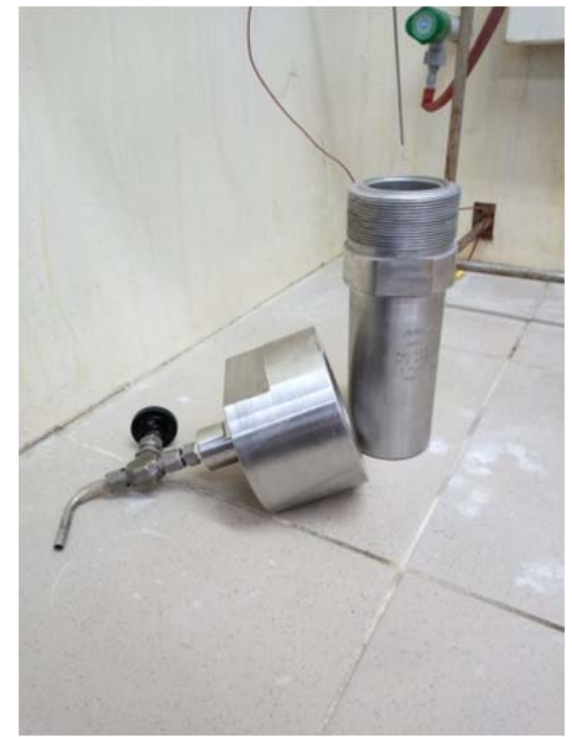

A high pressure reactor.

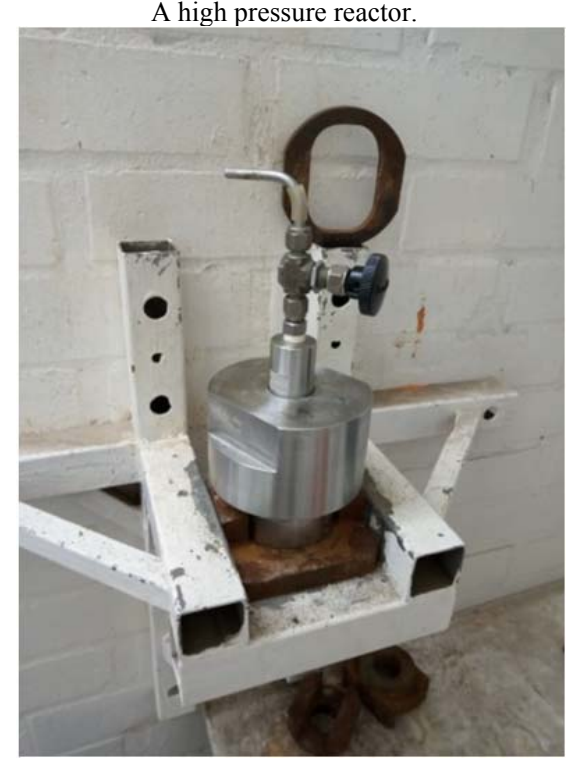

Fixing the high pressure reactor on the holder

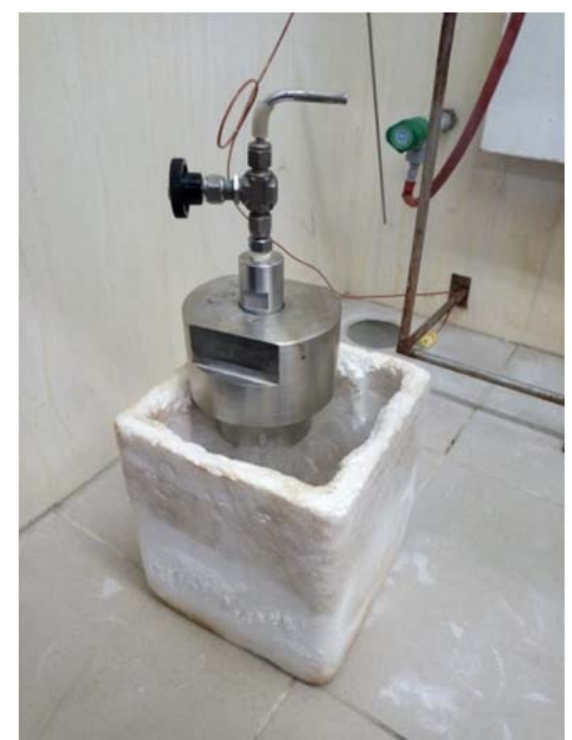

Cooling down the reaction mixture.

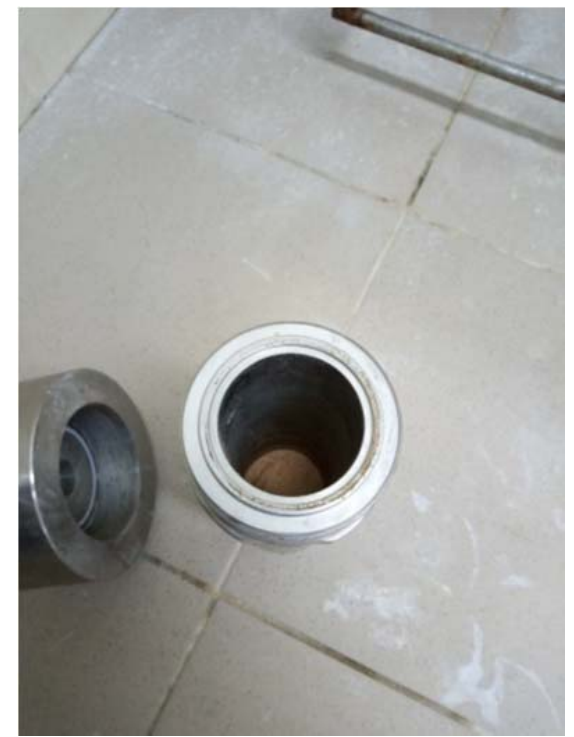

A high pressure reactor.

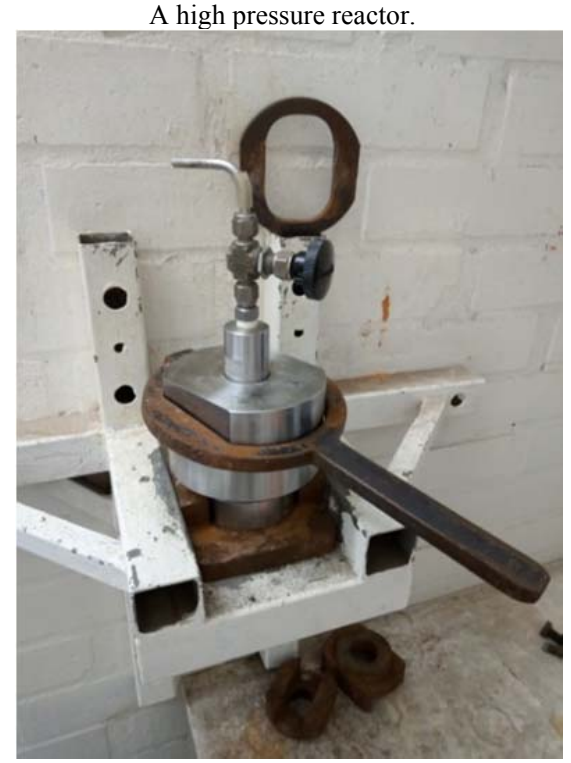

Installation of the high pressure reactor.

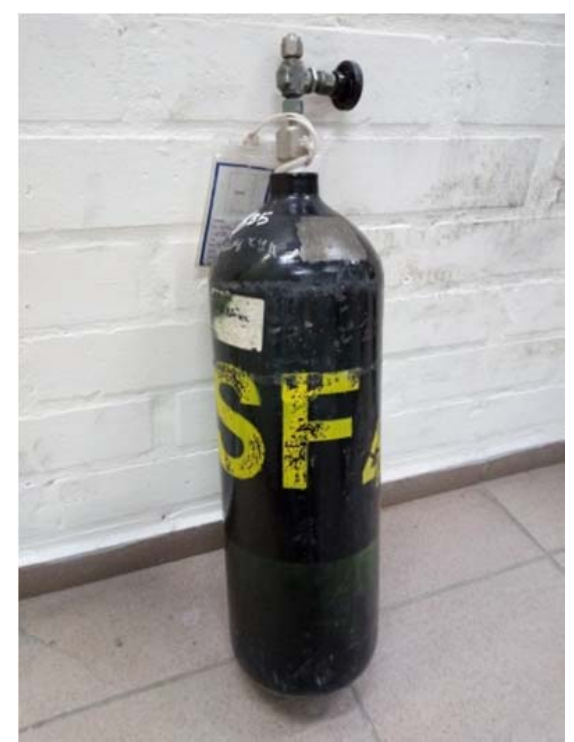

$\mathrm{SF}_{4}$
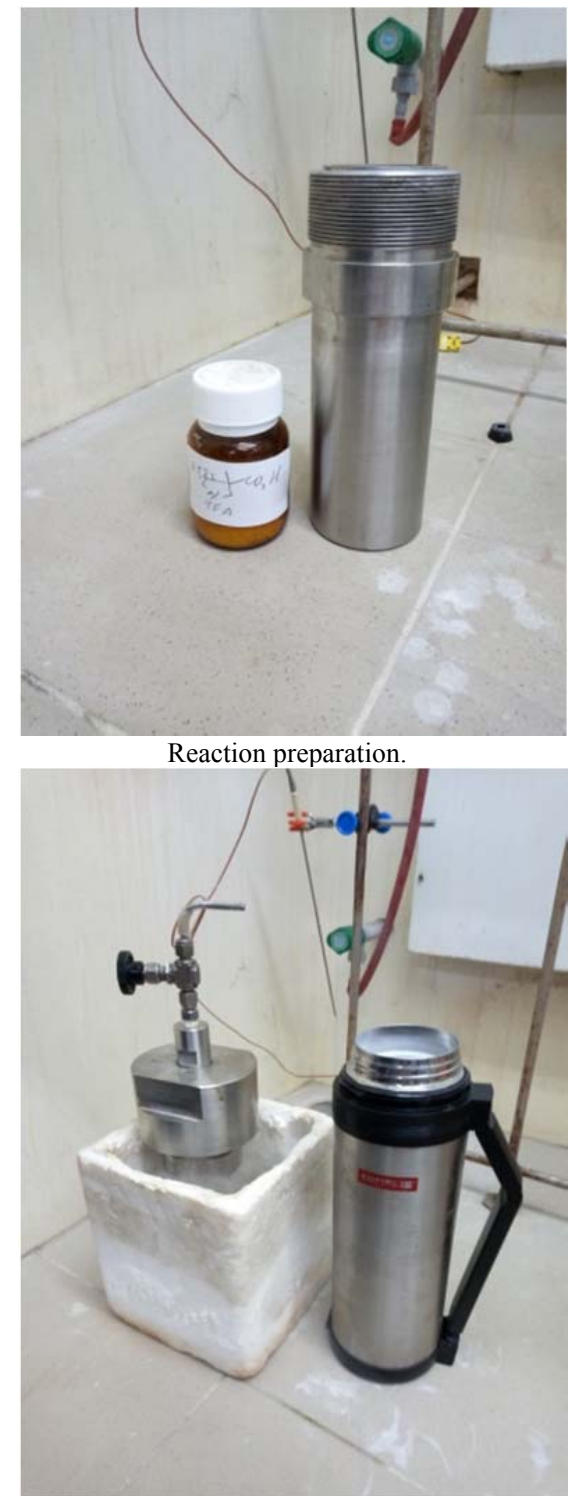

Freezing the reaction mixture with liquid

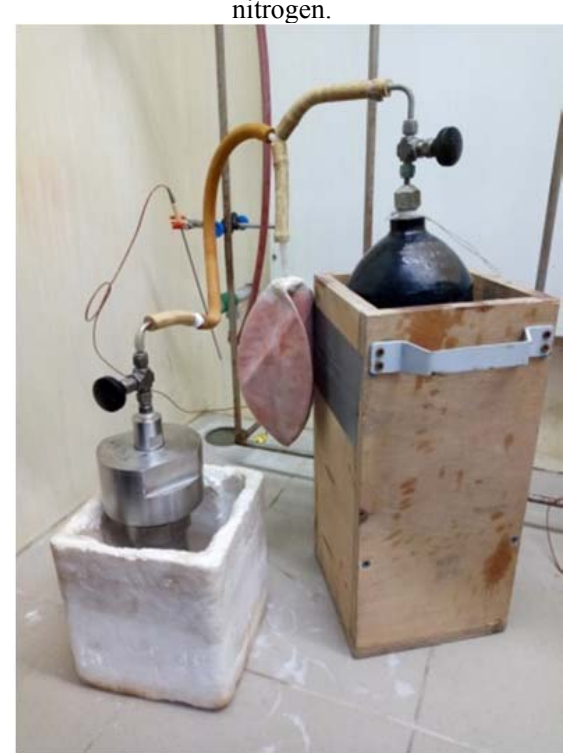

System for gas injection. 


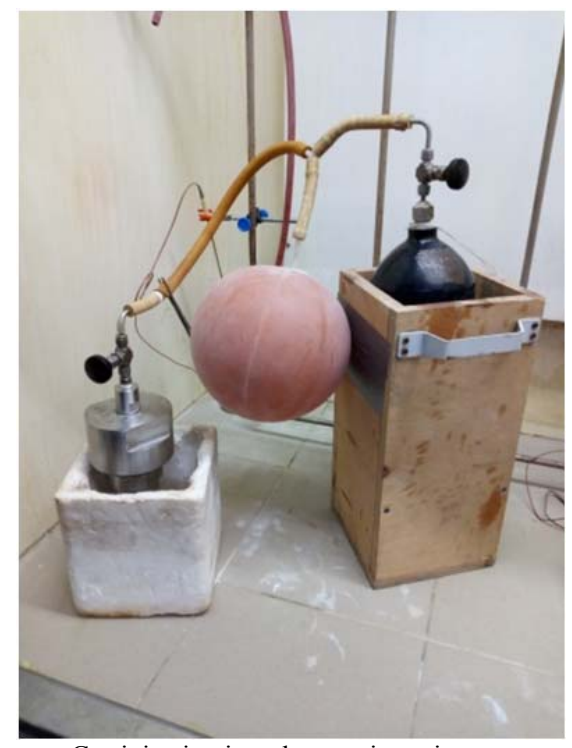

Gas injection into the reaction mixture
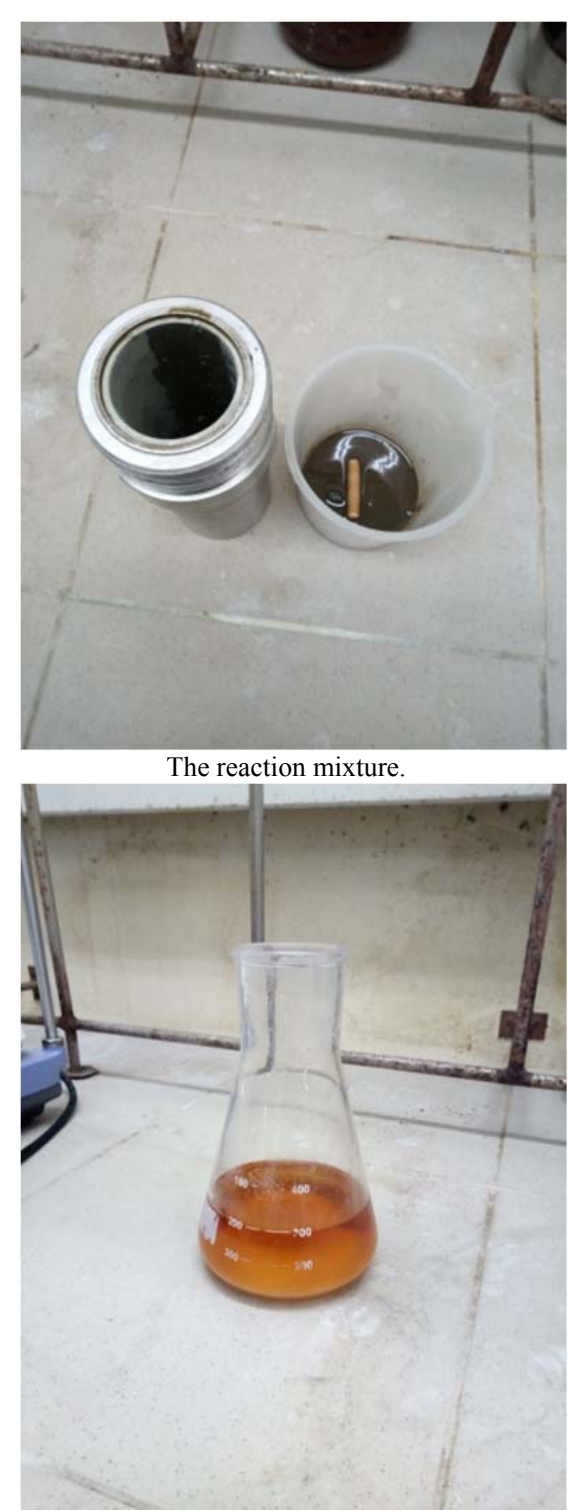

The combined organic layers.

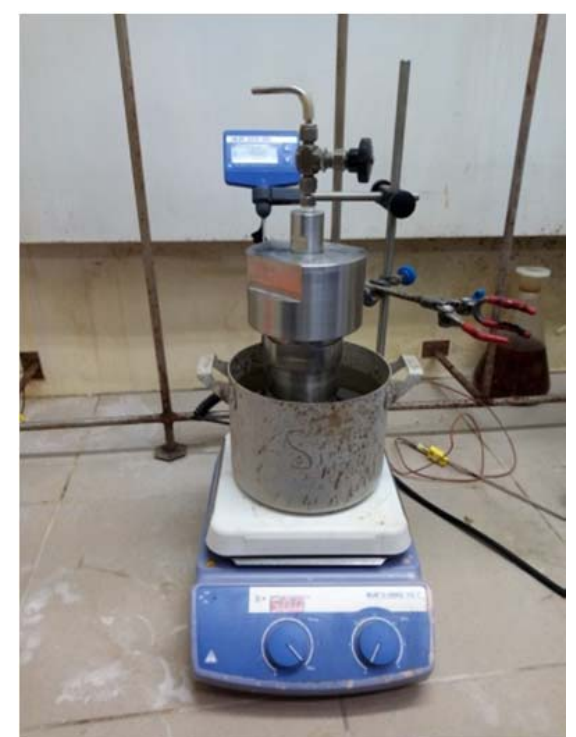

The high pressure reactor is placed in an oil bath, and heated for $24 \mathrm{~h}$ at $55^{\circ} \mathrm{C}$.

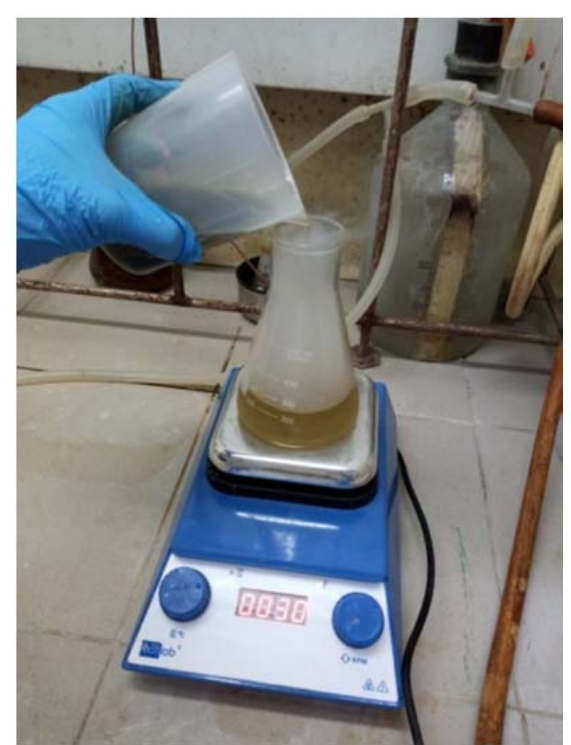

Mixing with $\mathrm{CH}_{2} \mathrm{Cl}_{2}$

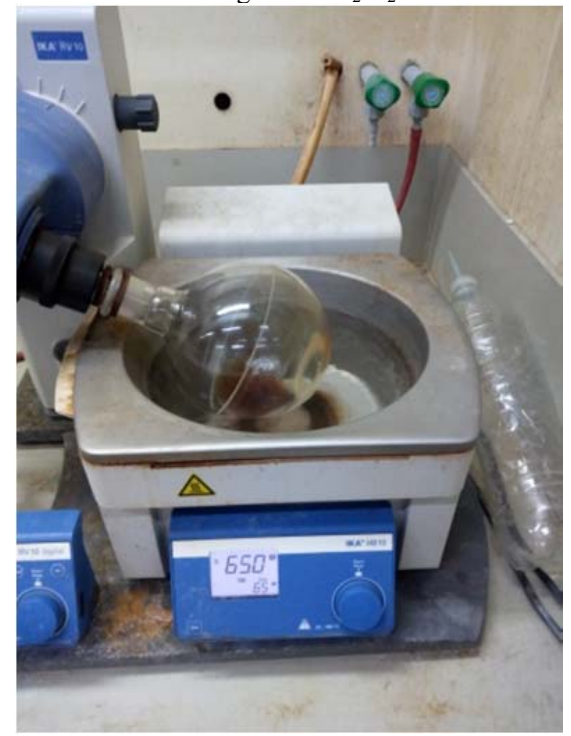

Evaporation in vacuo.

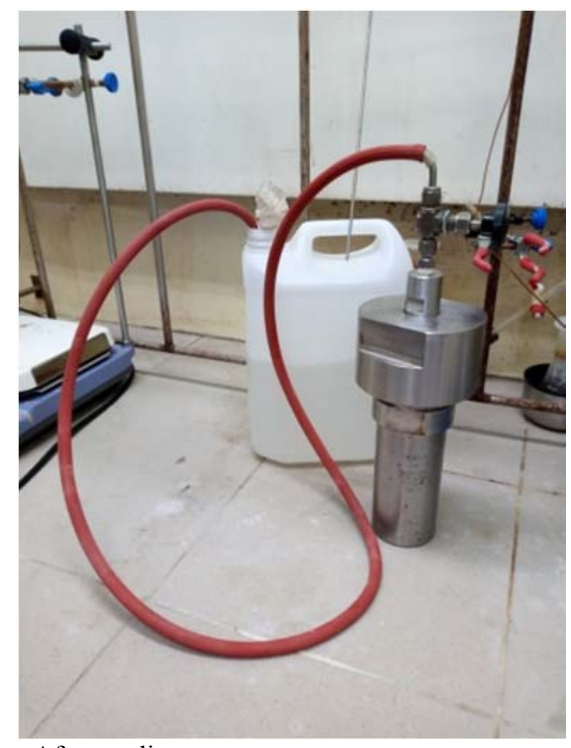

After cooling to a room temperature, gaseous products are vented of into an aqueous solution of $\mathrm{NaOH}$.

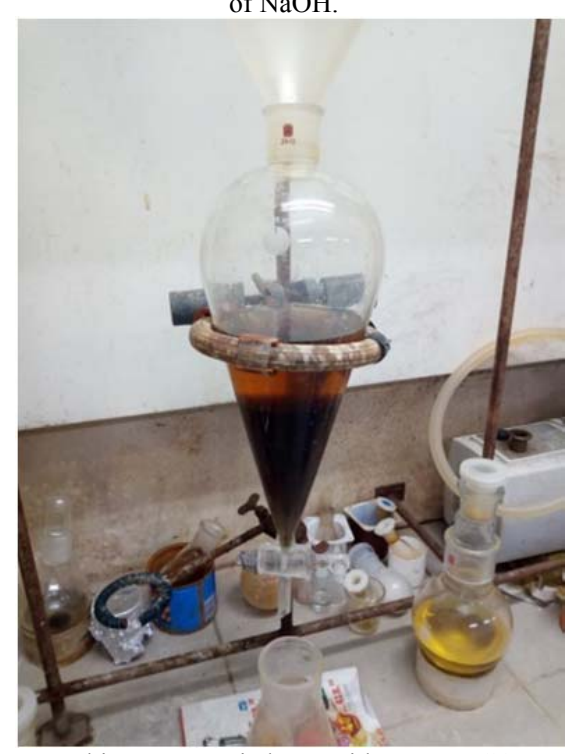

Washing an organic layer with aq. $\mathrm{NaHCO}_{3}$.

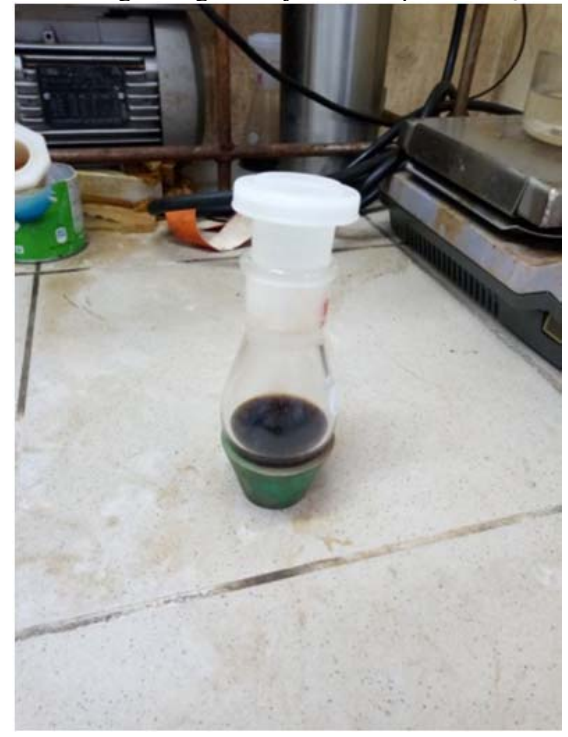

Crude product. 


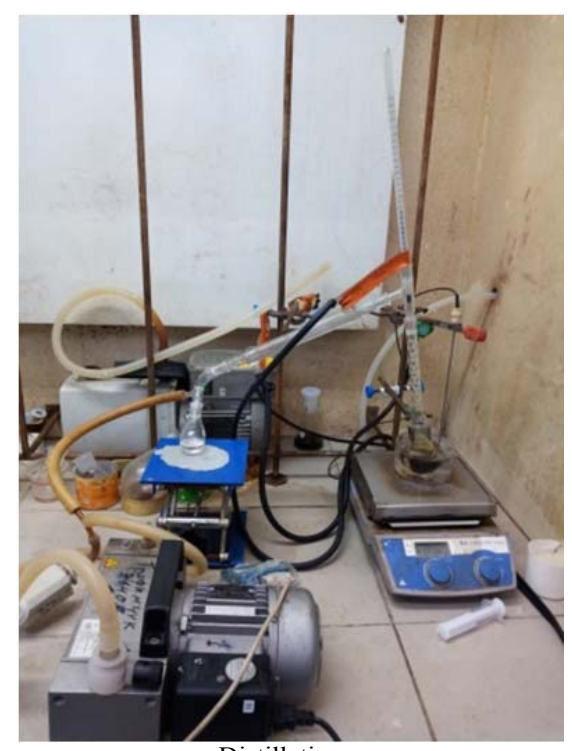

Distillation.

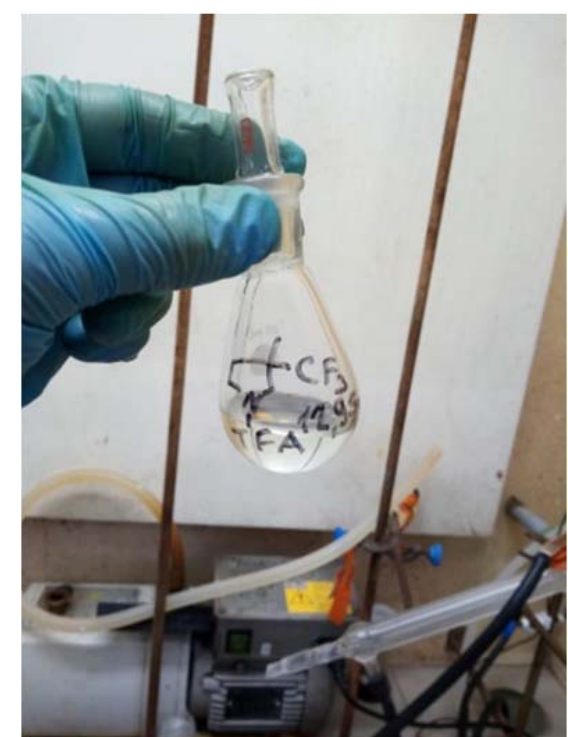

The pure final product. 


\section{Compound 2a}

tp11007

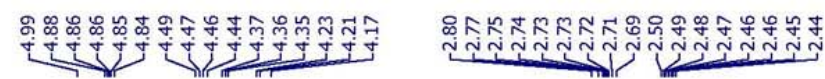
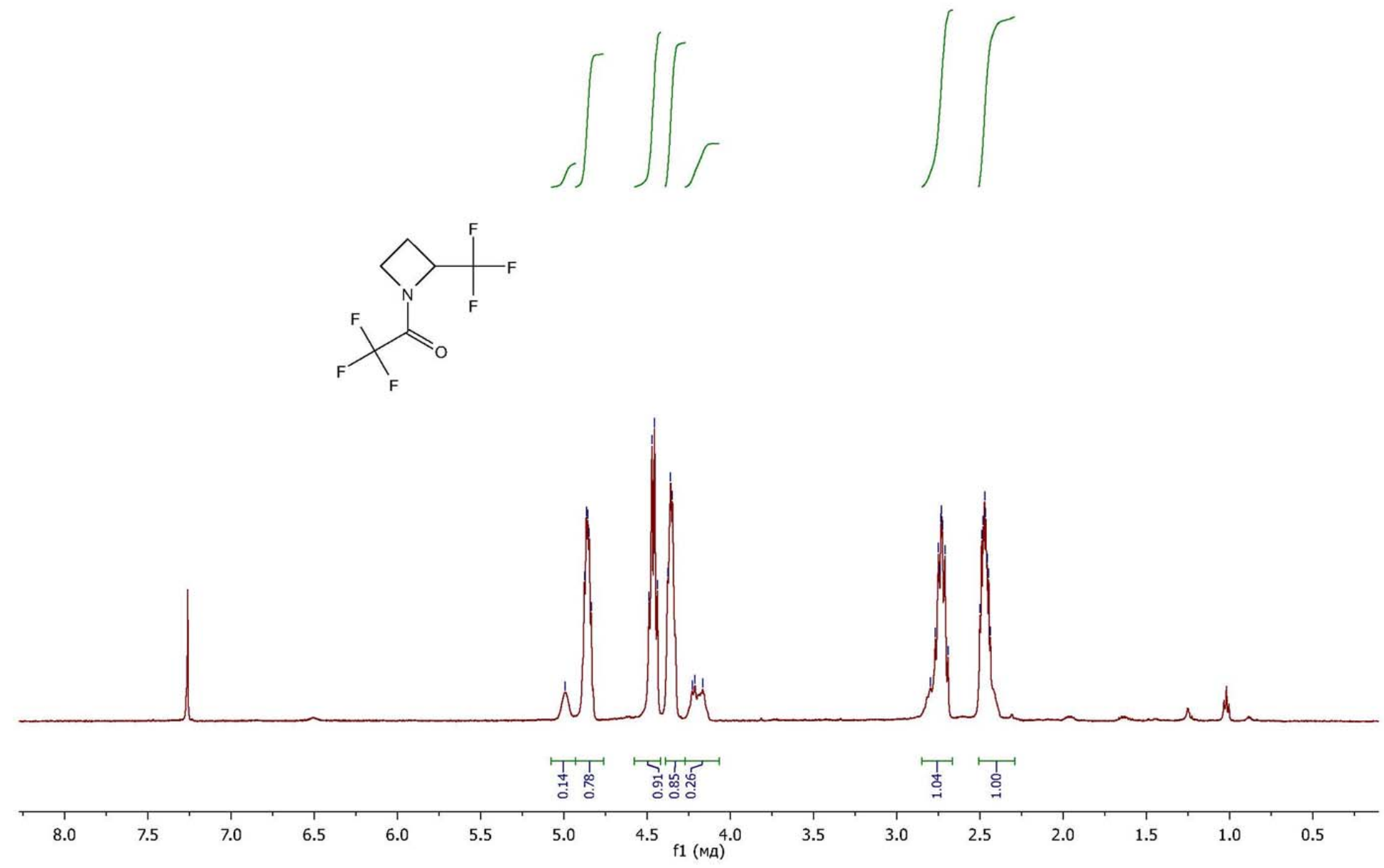
tp11007a_C13APT

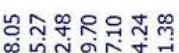

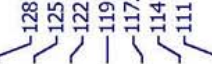

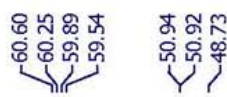

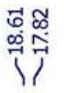

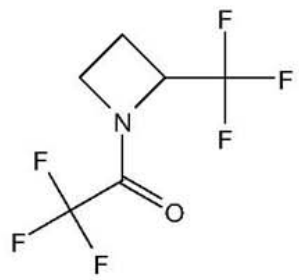

(2)

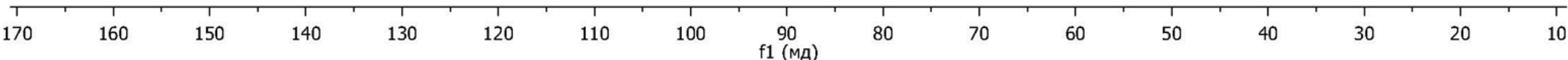


tp11007_F19\{H\}

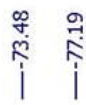

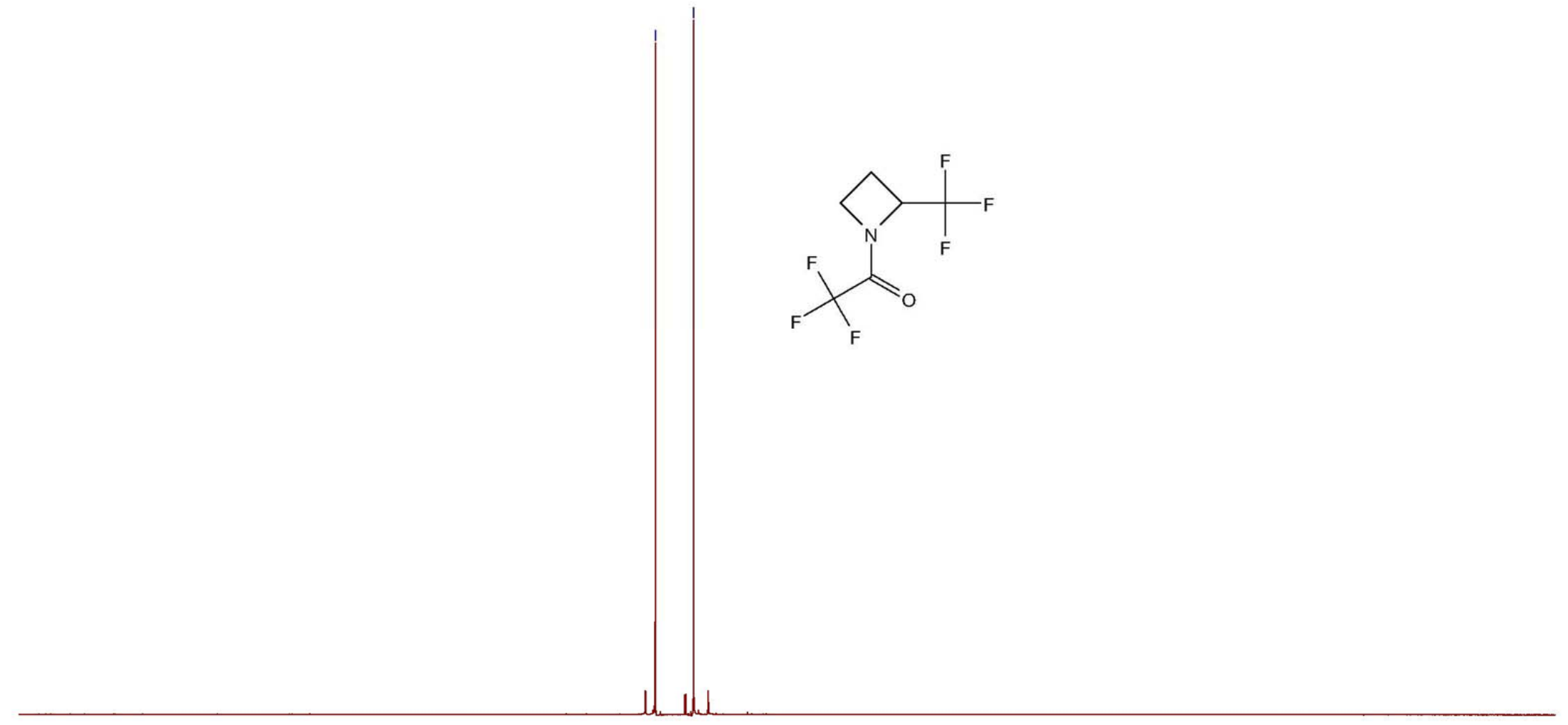

\begin{tabular}{lllllllllllllllllllllllllllllllllllllll}
\hline-15 & -20 & -25 & -30 & -35 & -40 & -45 & -50 & -55 & -60 & -65 & -70 & -75 & -80 & -85 & -90 & -95 & -105 & -115 & -125 & -135 & -145 & -155 &
\end{tabular}




\section{Compound 3a}

R979003

$\mathrm{Br} \sim \mathrm{CF}_{3}$

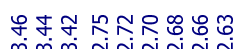

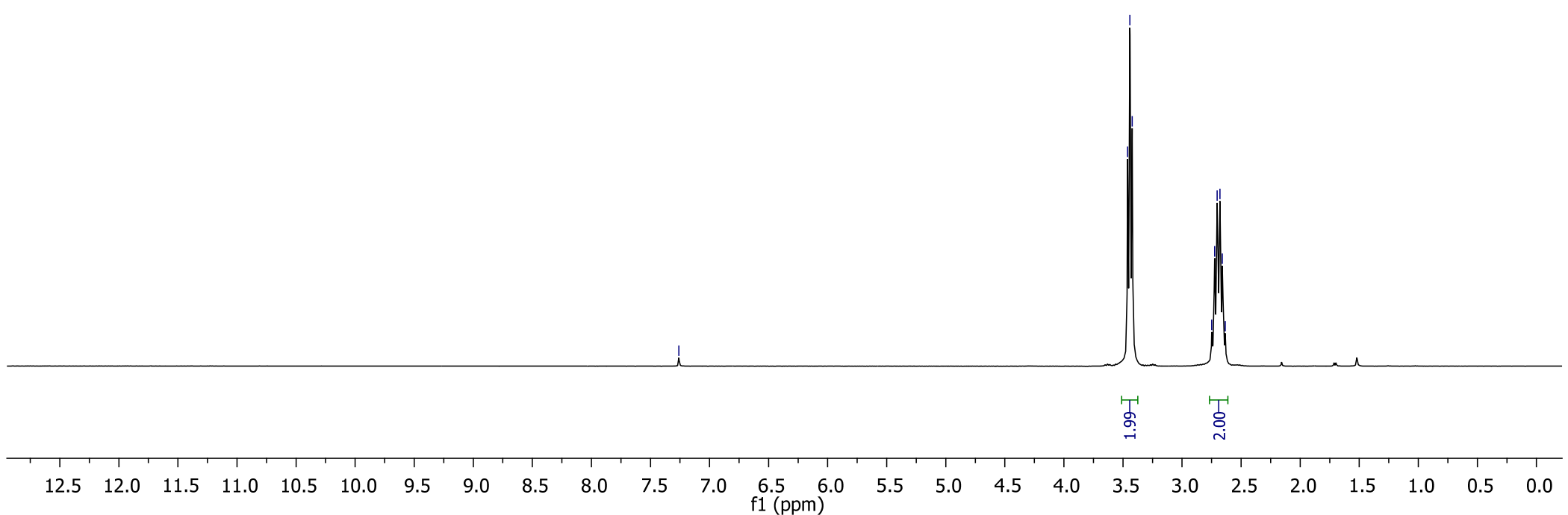


$\mathrm{Br} \sim \mathrm{CF}_{3}$

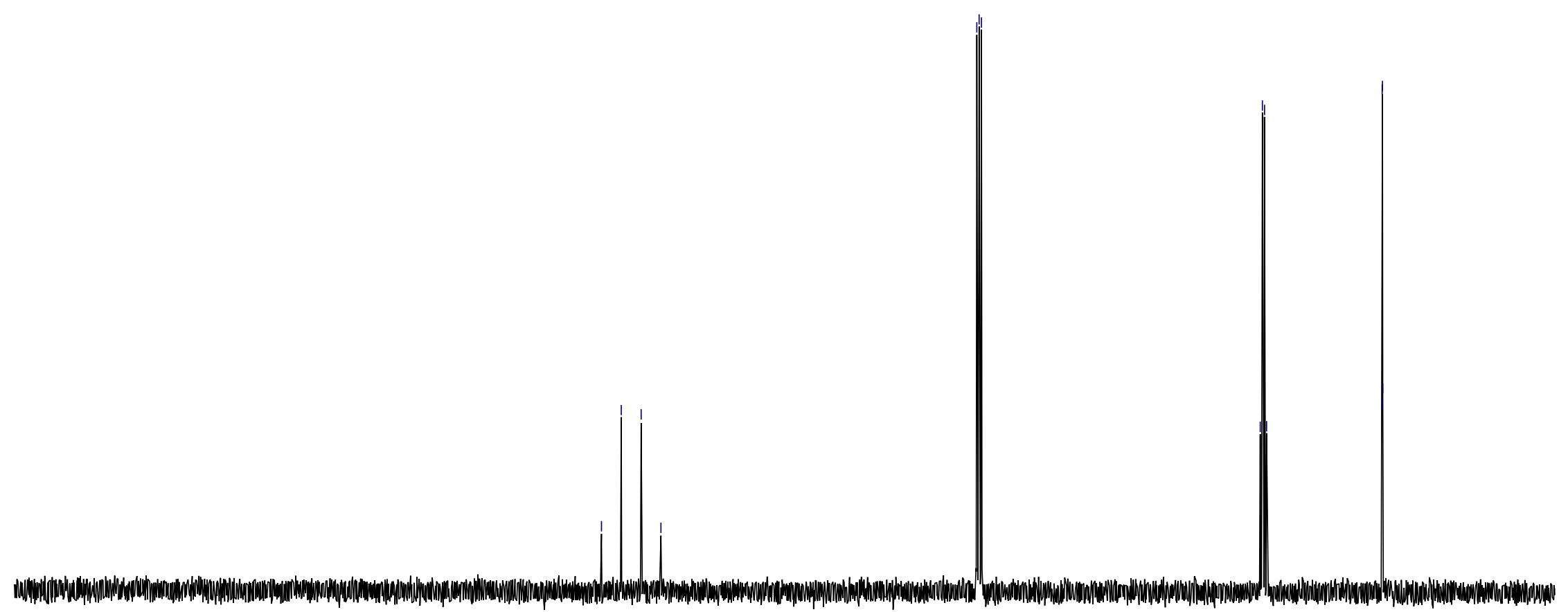


R979003_F19 $\{H\}$

$\mathrm{Br} \sim \mathrm{CF}_{3}$

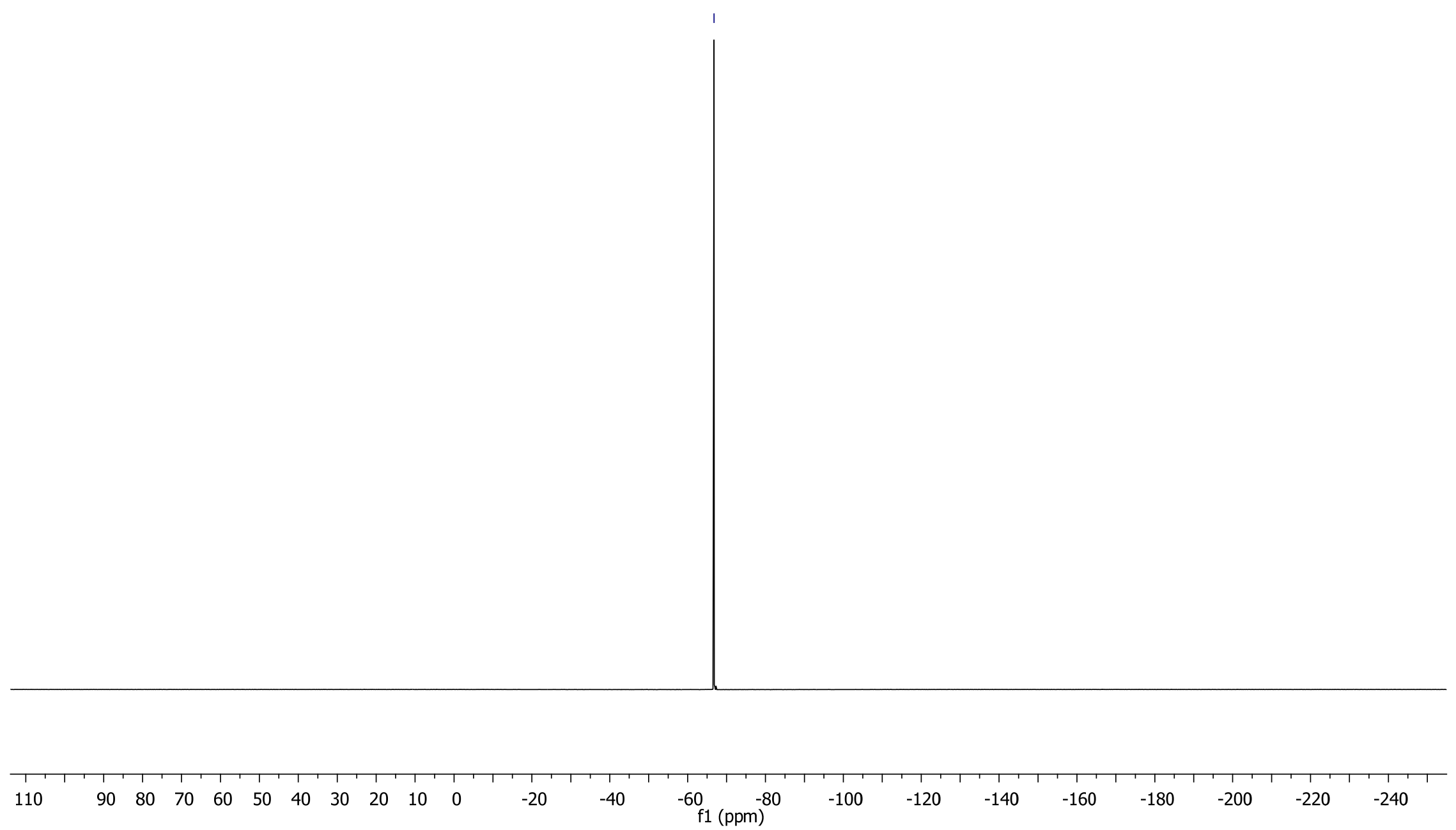




\section{Compound 4a}

R1135235

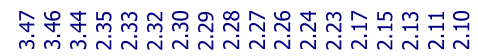

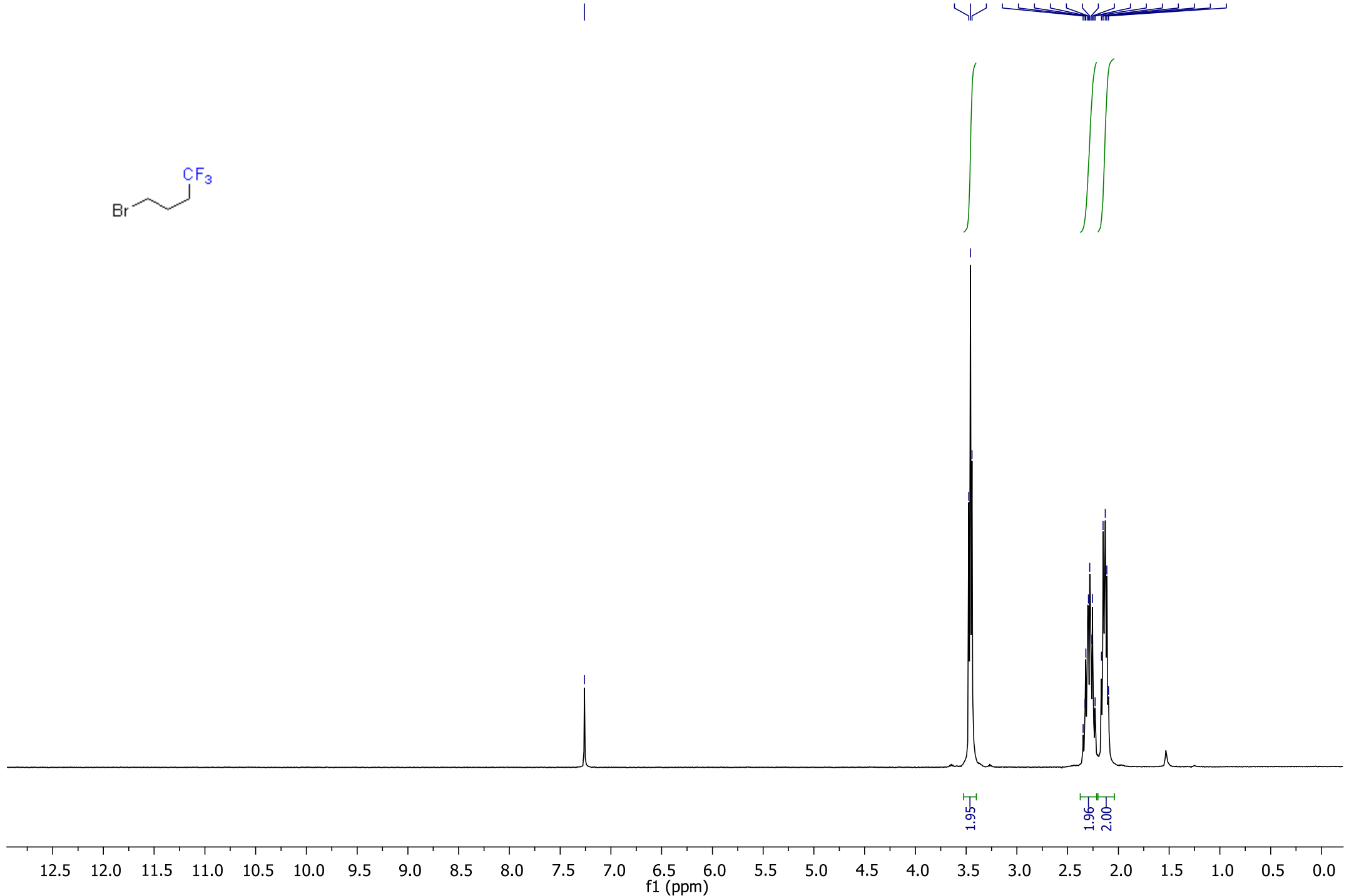


R1135235_C13

$\sim_{\mathrm{Br}}^{\mathrm{CF}_{3}}$

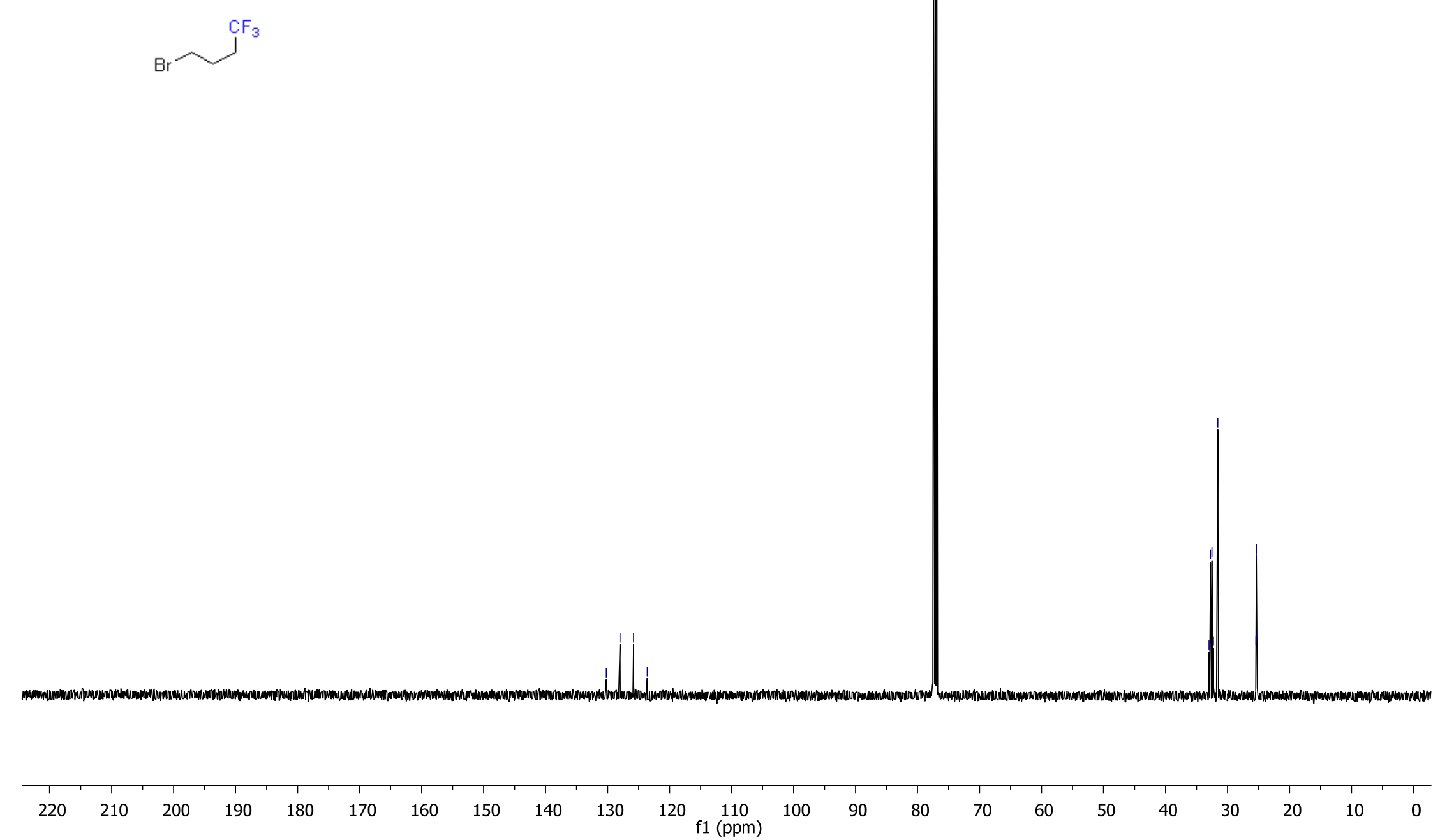


R1135235_F19 $\{H\}$

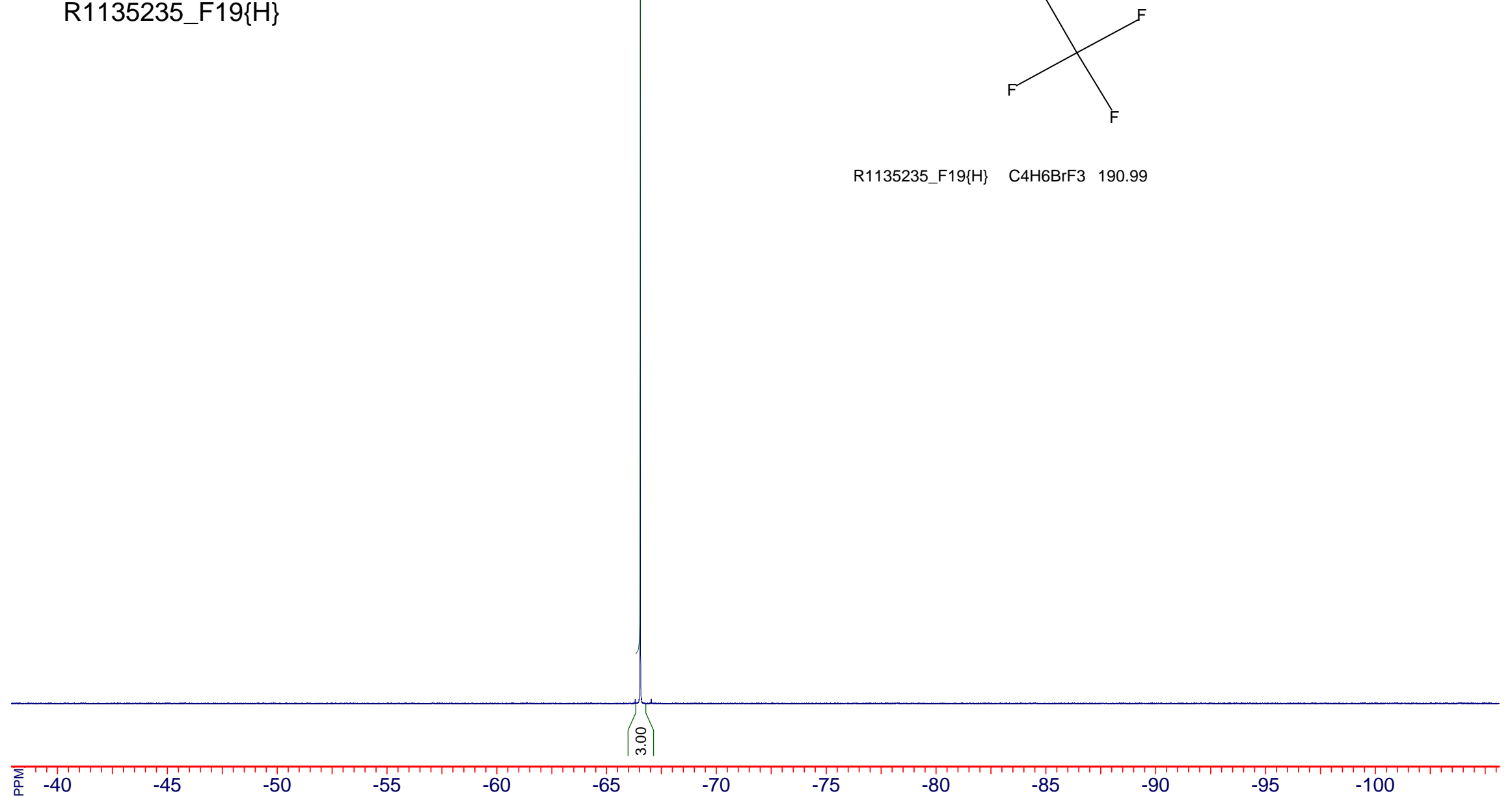




\section{Compound 5a}

R417038

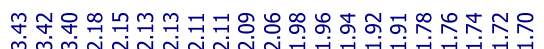

$\mathrm{Br} \mathrm{j}^{\mathrm{CF} \mathrm{F}_{3}}$
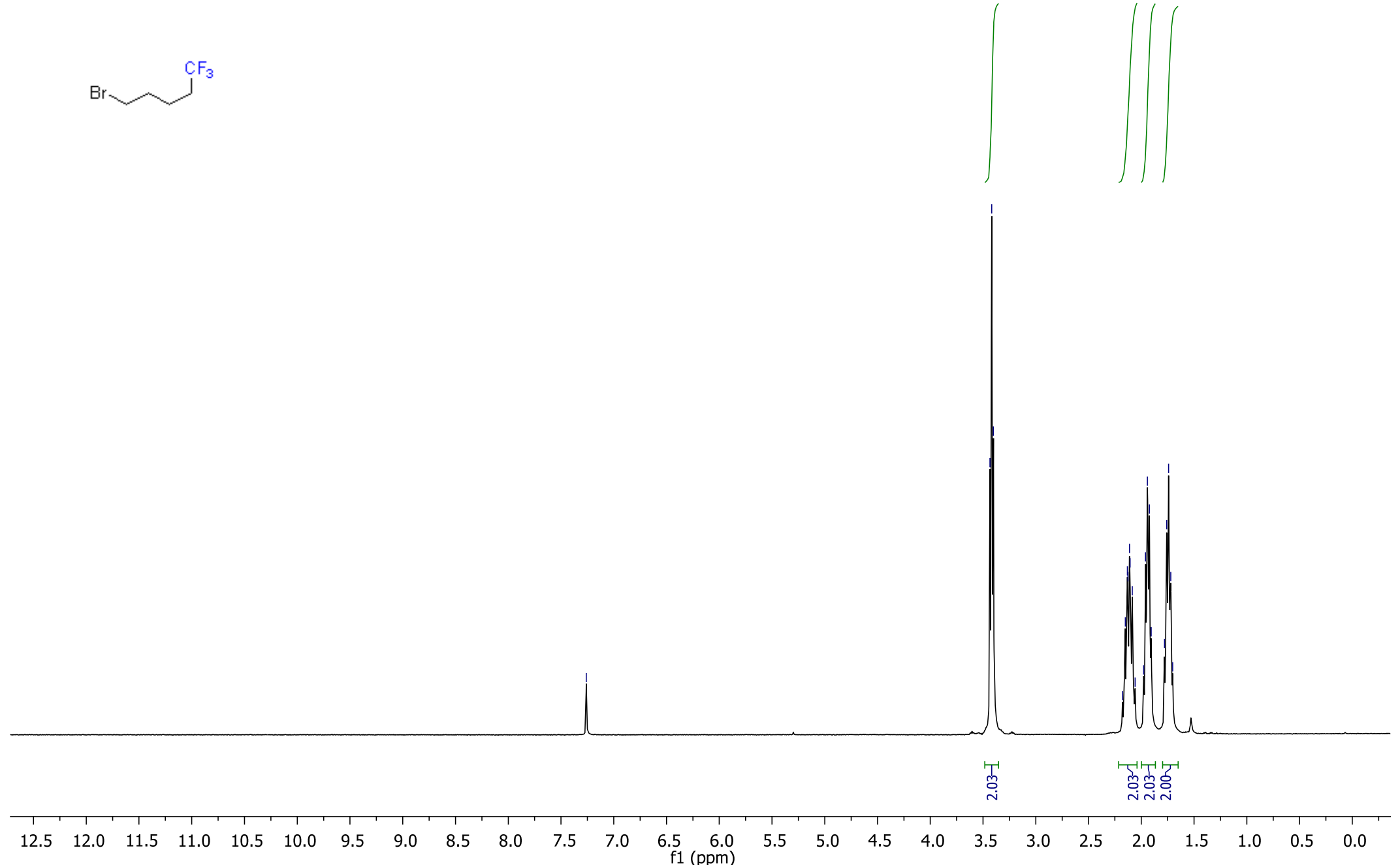
R417038_C1

$\mathrm{Br} \mathrm{CF}^{\mathrm{CF}_{3}}$

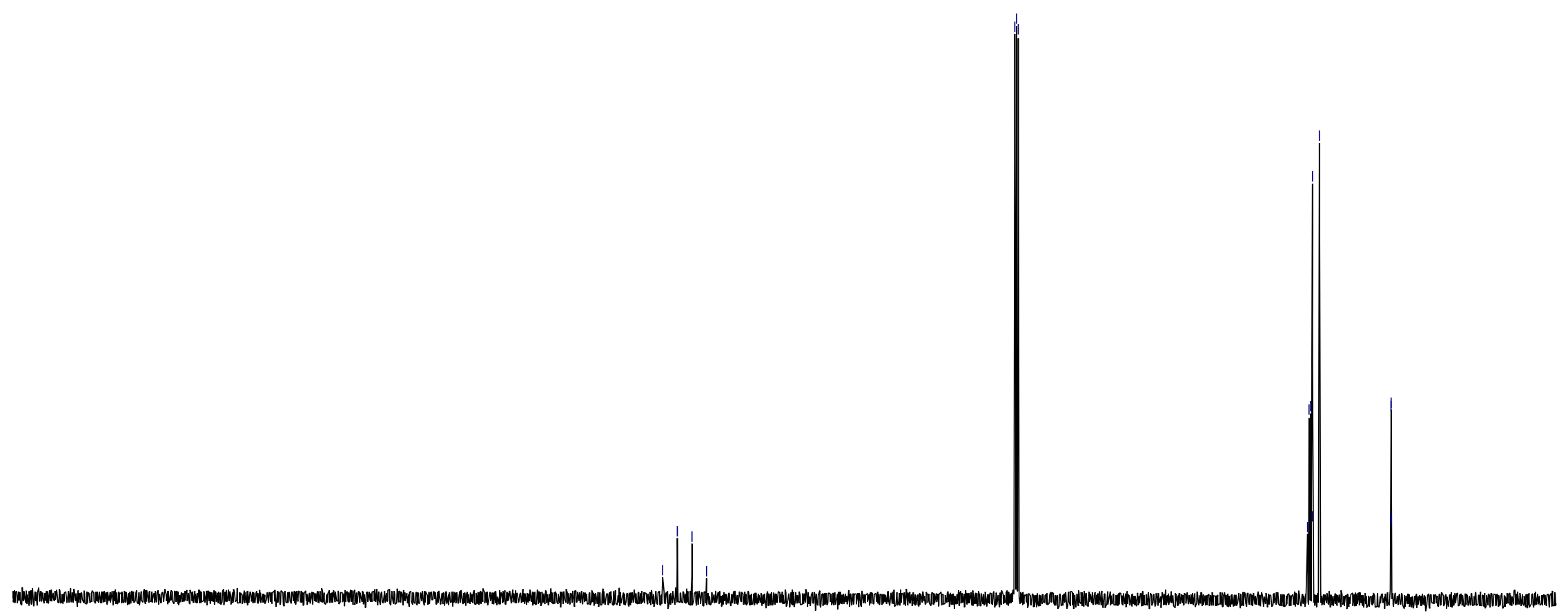

220 
R417038 F19 $\{\mathrm{H}\}$

$19 F-\{1 \mathrm{H}\}$

$\mathrm{Br} \sim^{C F_{3}}$

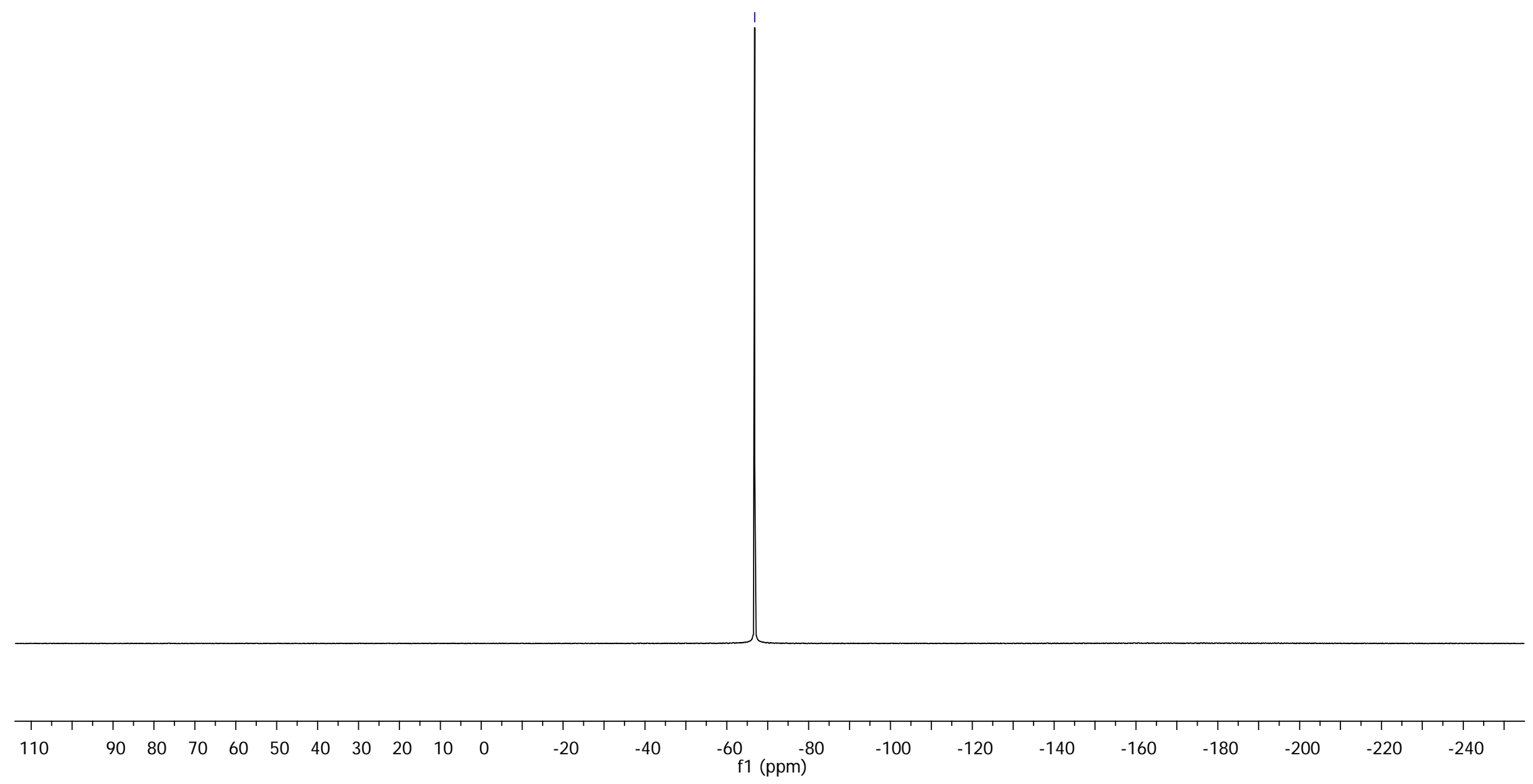




\section{Compound 6a}

mom-CF3CH2a

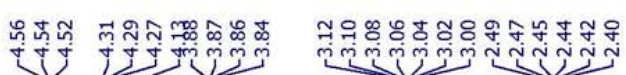

$\iiint \int 5$

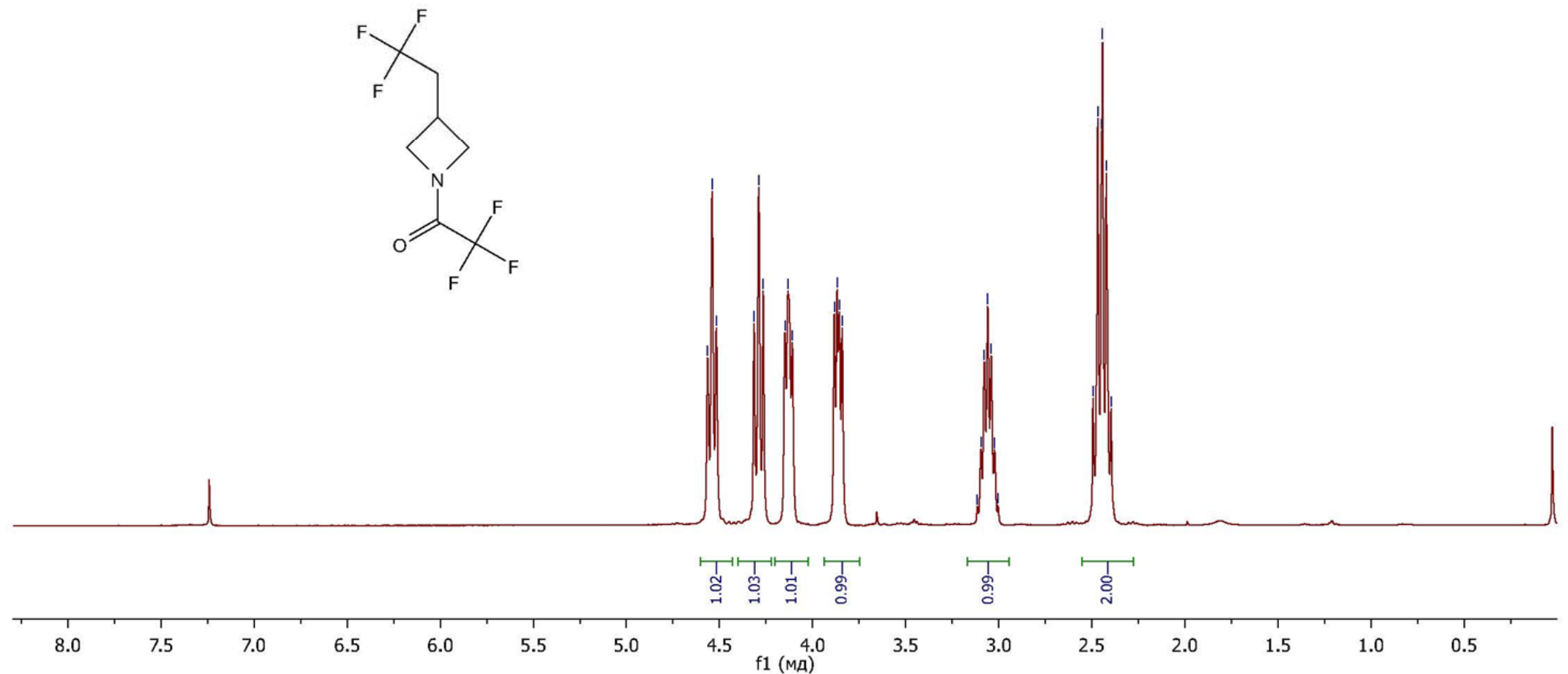



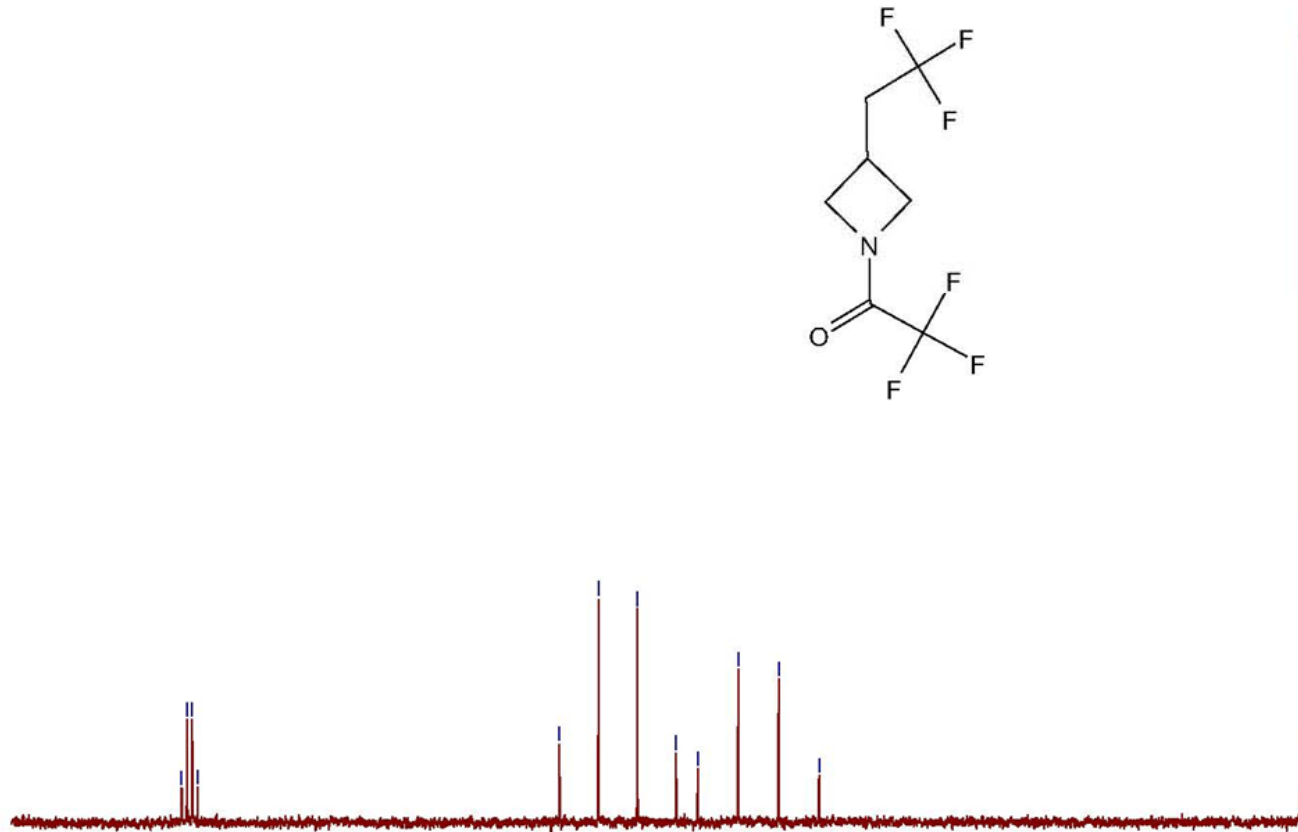
mom-CF3CH2a-F19
$19 \mathrm{~F}$
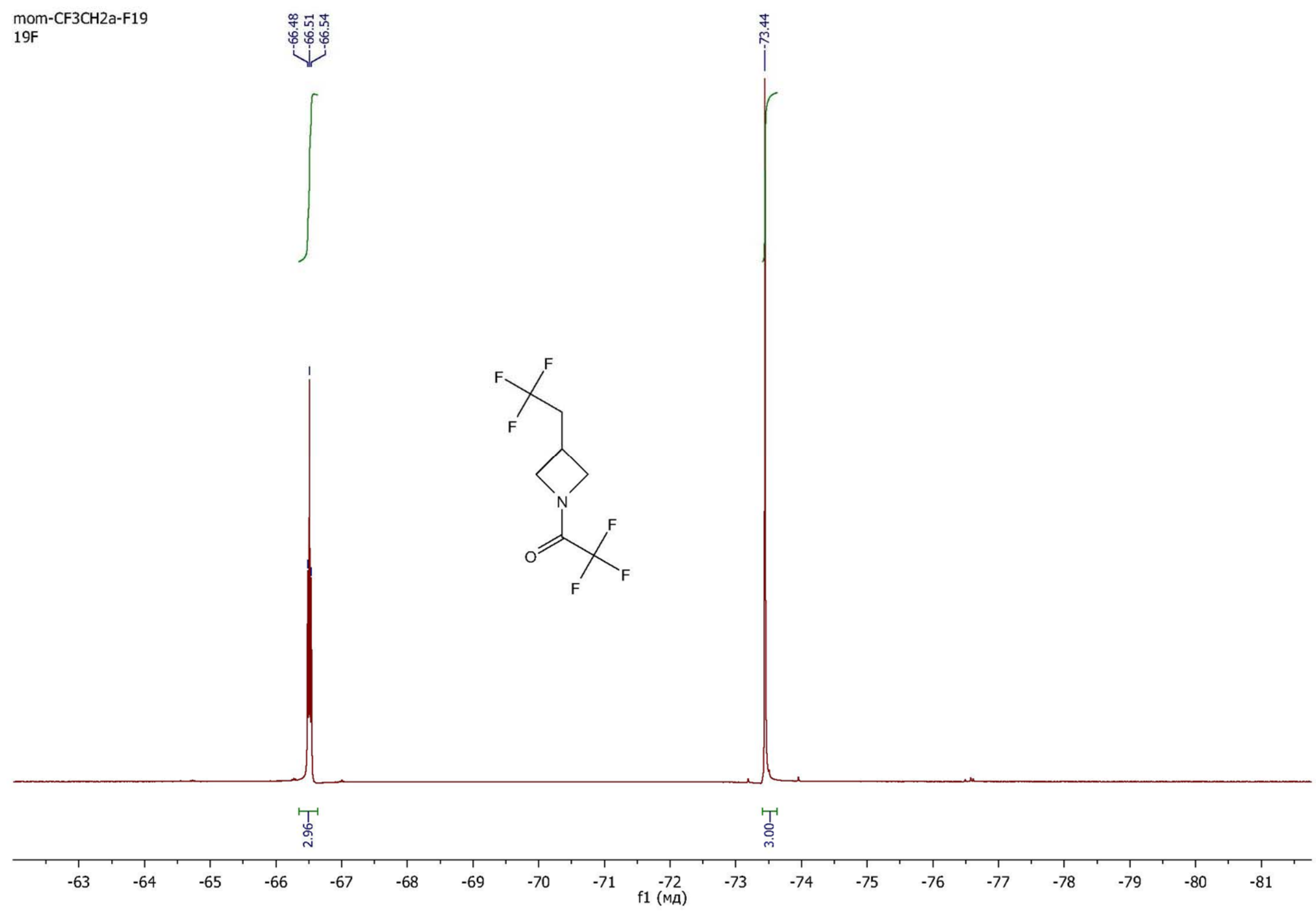

S21 


\section{Compound 7a}

tp1524؟

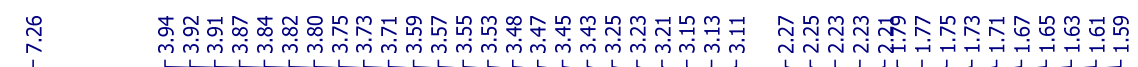
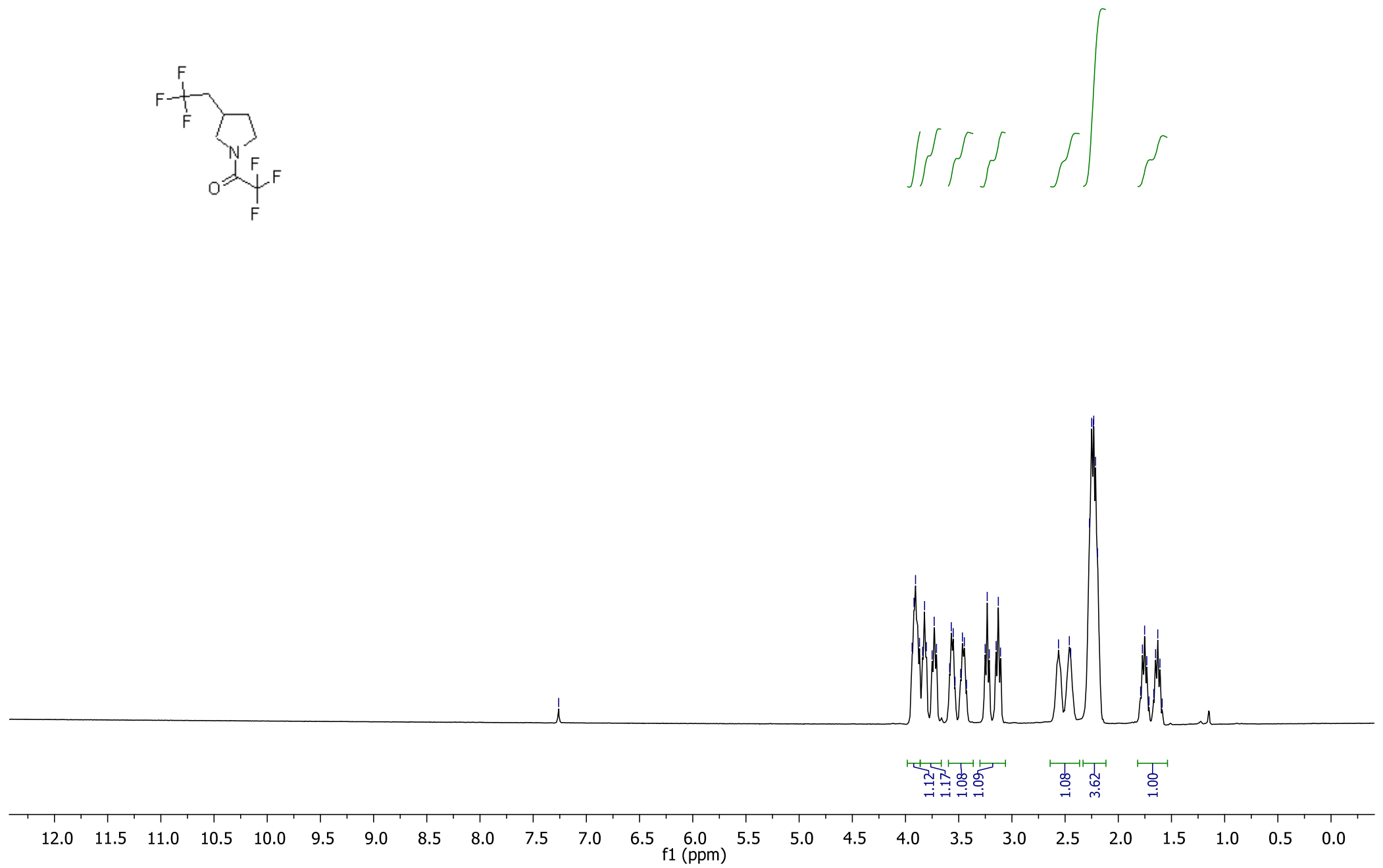

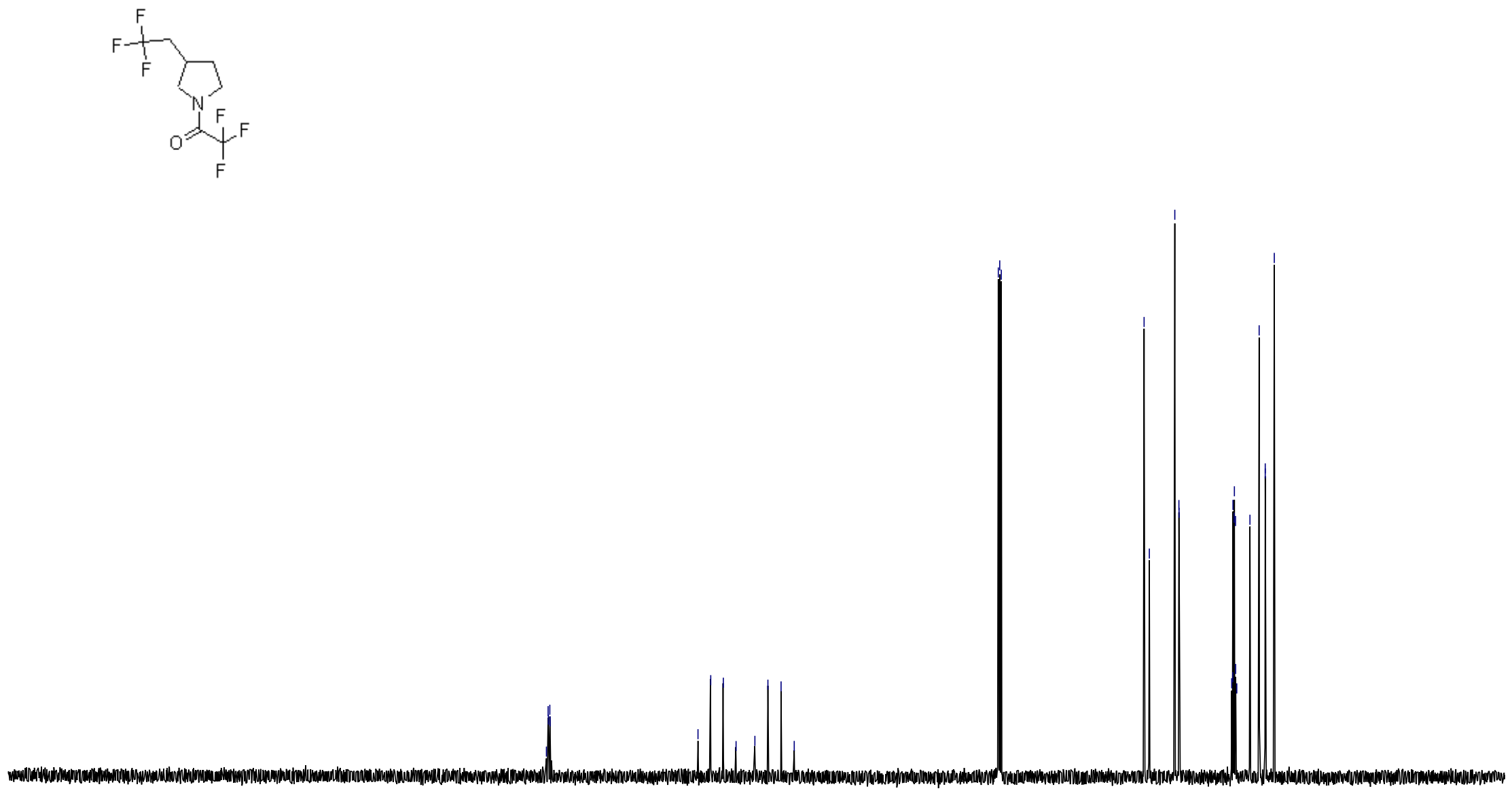

$240 \quad 230$ 


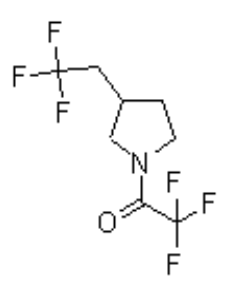




\section{Compound 8a}

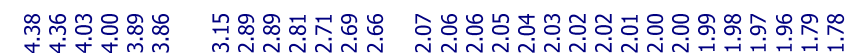

$\checkmark V H^{m i n i n}$

促
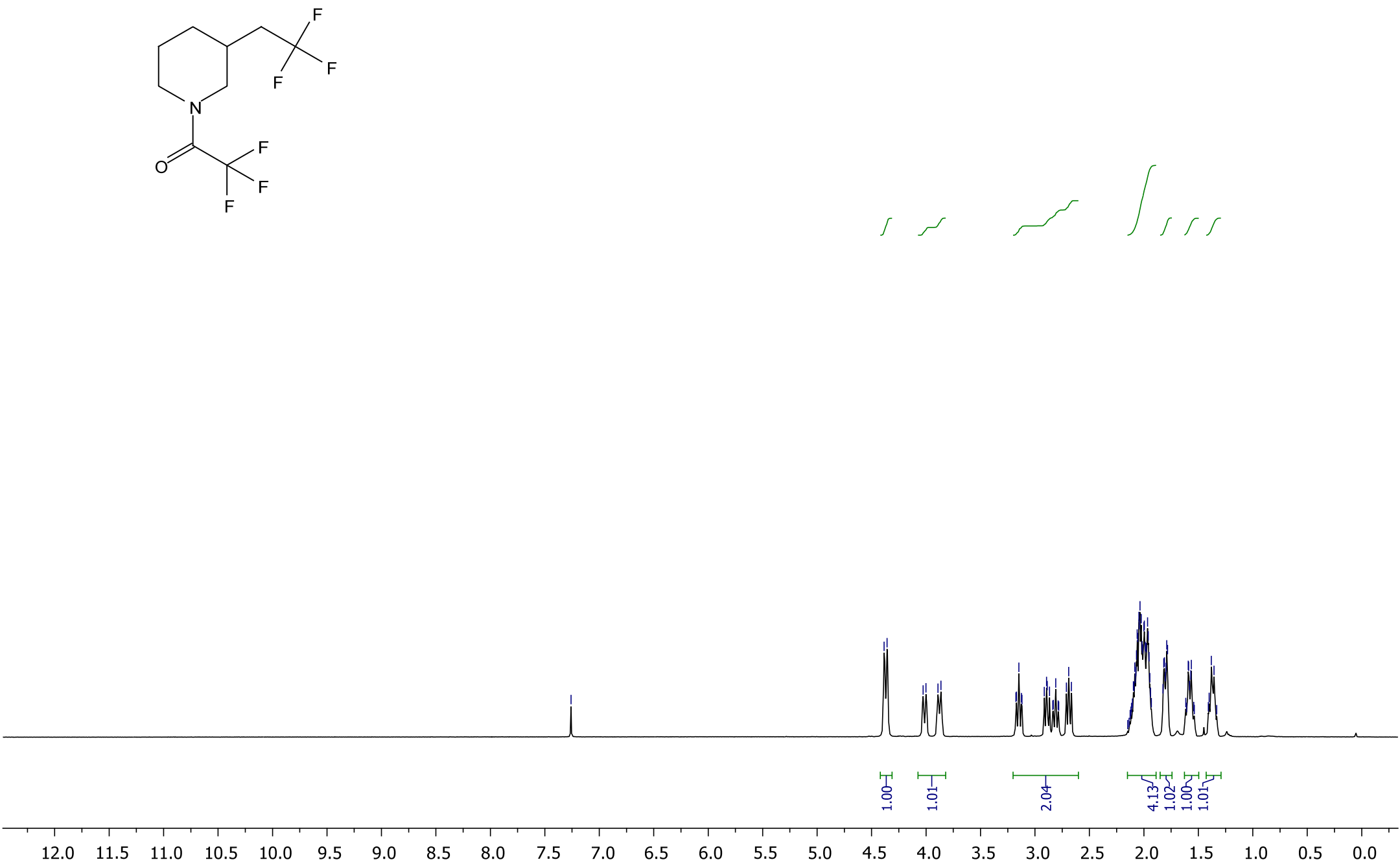


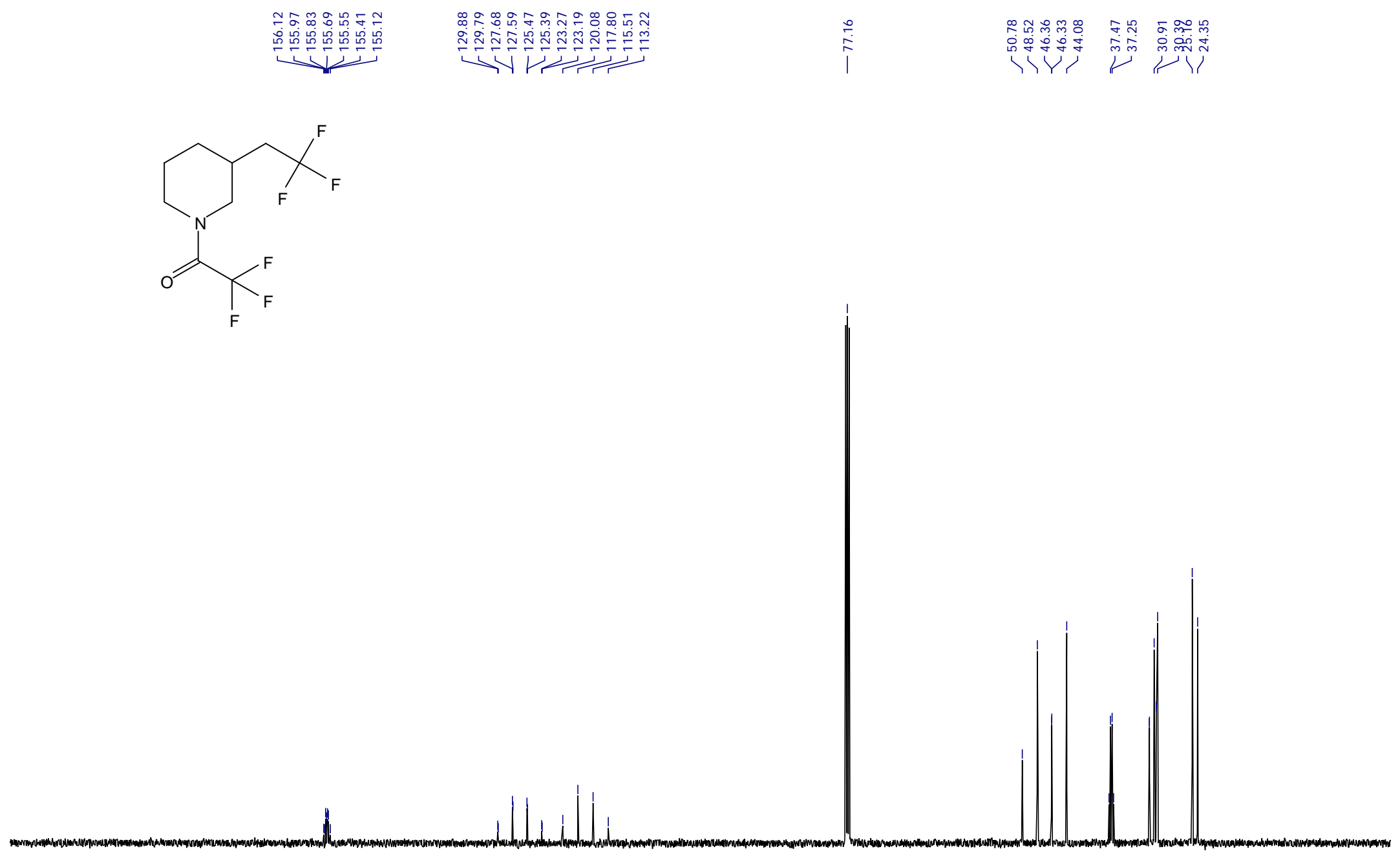




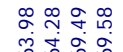

نิ
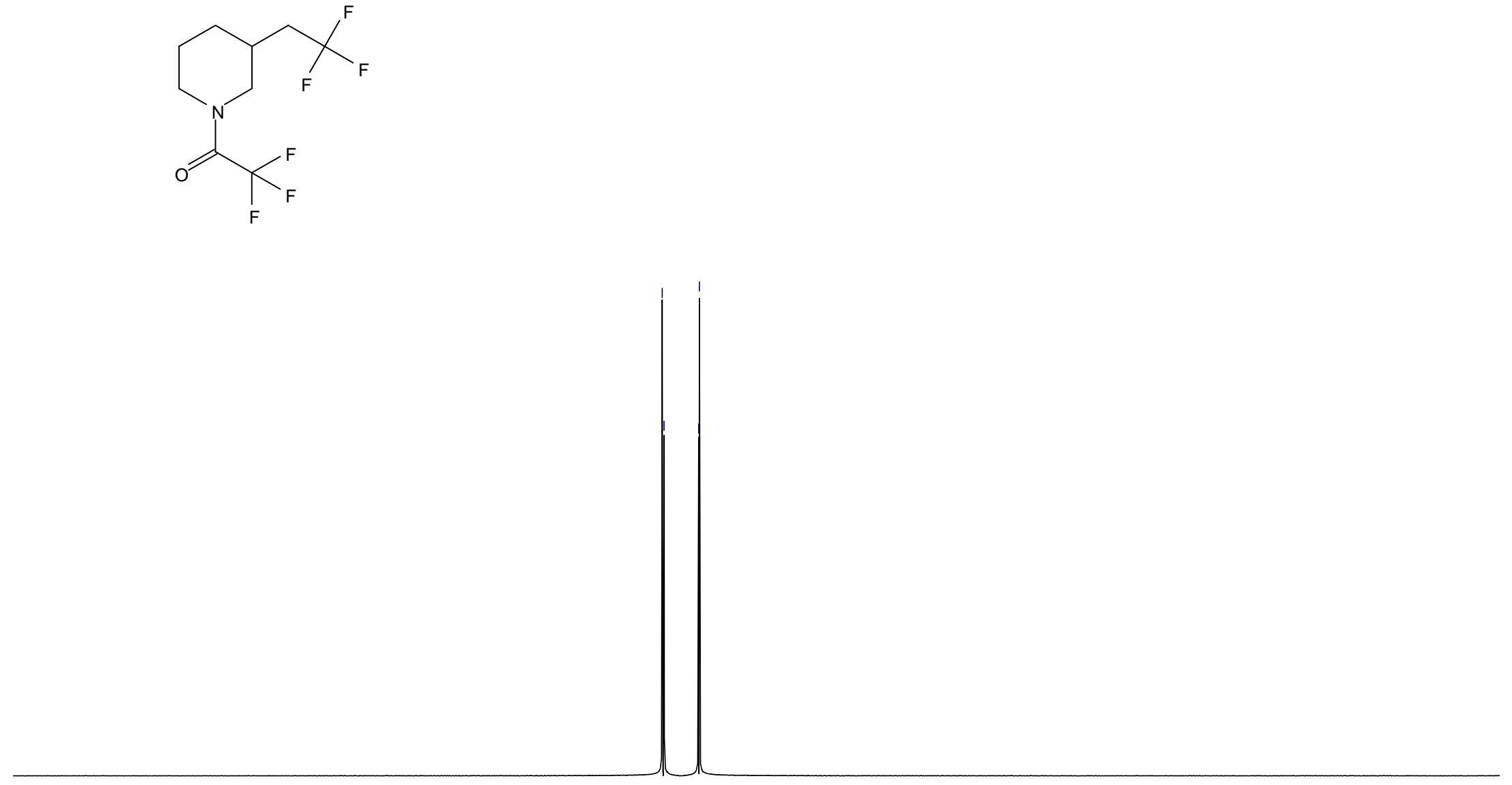

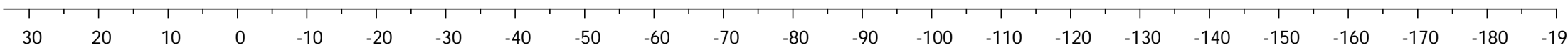




\section{Compound 9a}

tp1177i

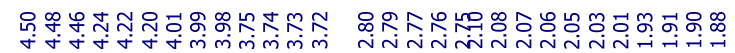
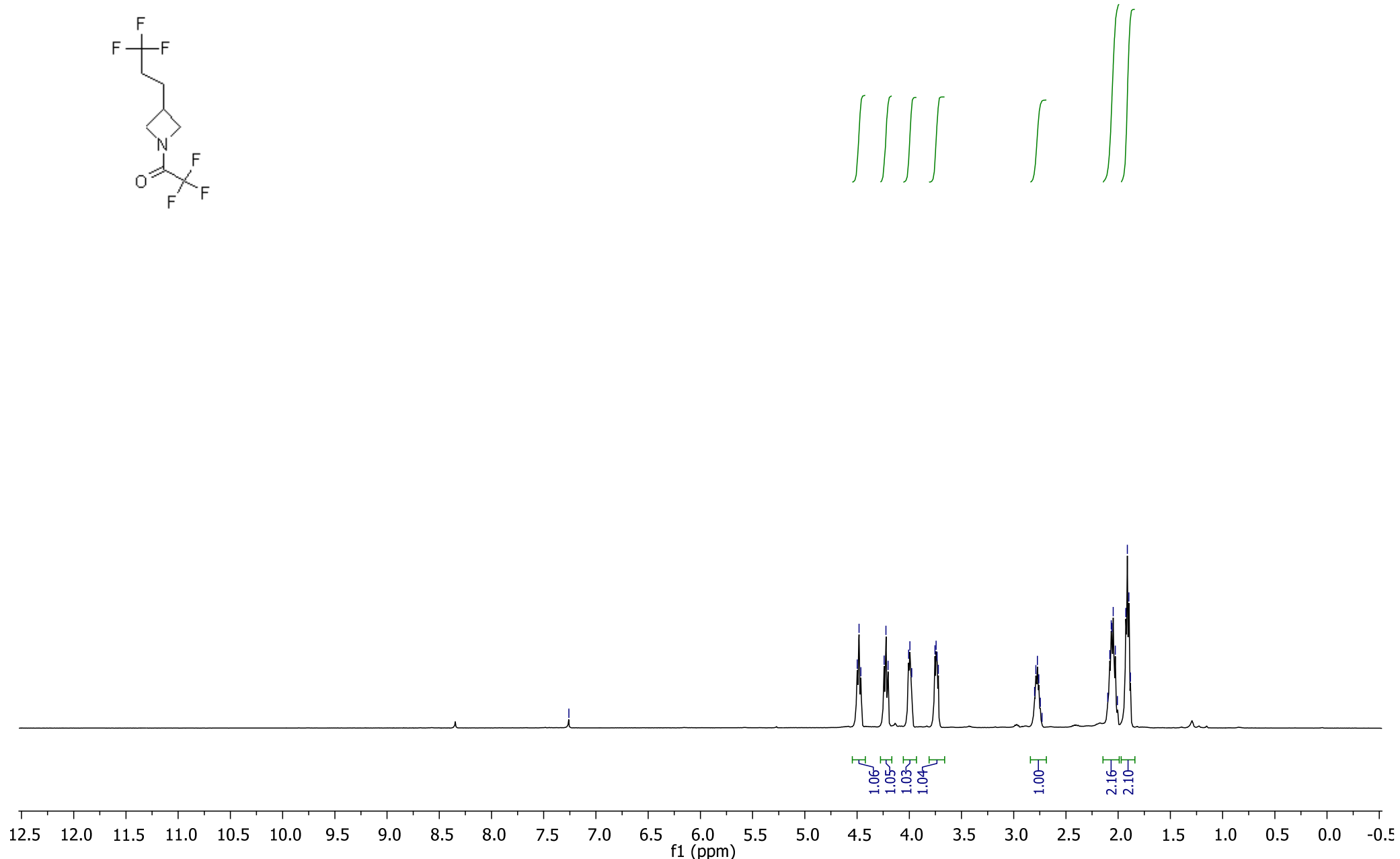
tp11777_C13

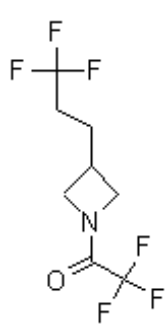

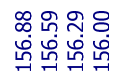

正

कू⿻日禸

11111111

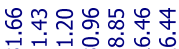

$\sqrt{1 / 1 i \pi}$

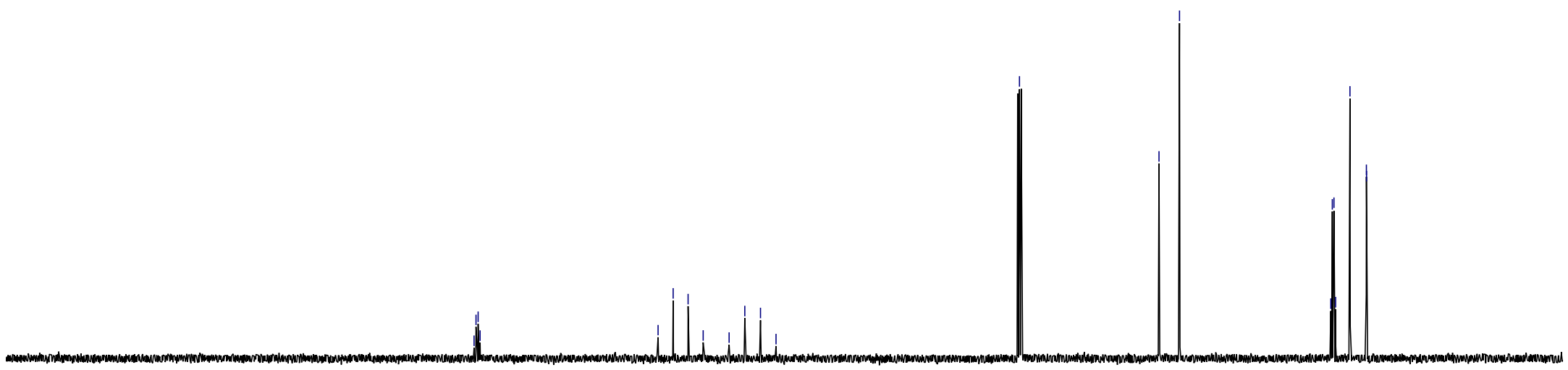

$\begin{array}{llllllllllll}220 & 210 & 200 & 190 & 180 & 170 & 160 & 150 & 140 & 130 & 120 & 110\end{array}$ f1 (ppm) 
tp11777_F19 $\{\mathrm{H}\}$

$19 \mathrm{~F}-\{1 \mathrm{H}\}$
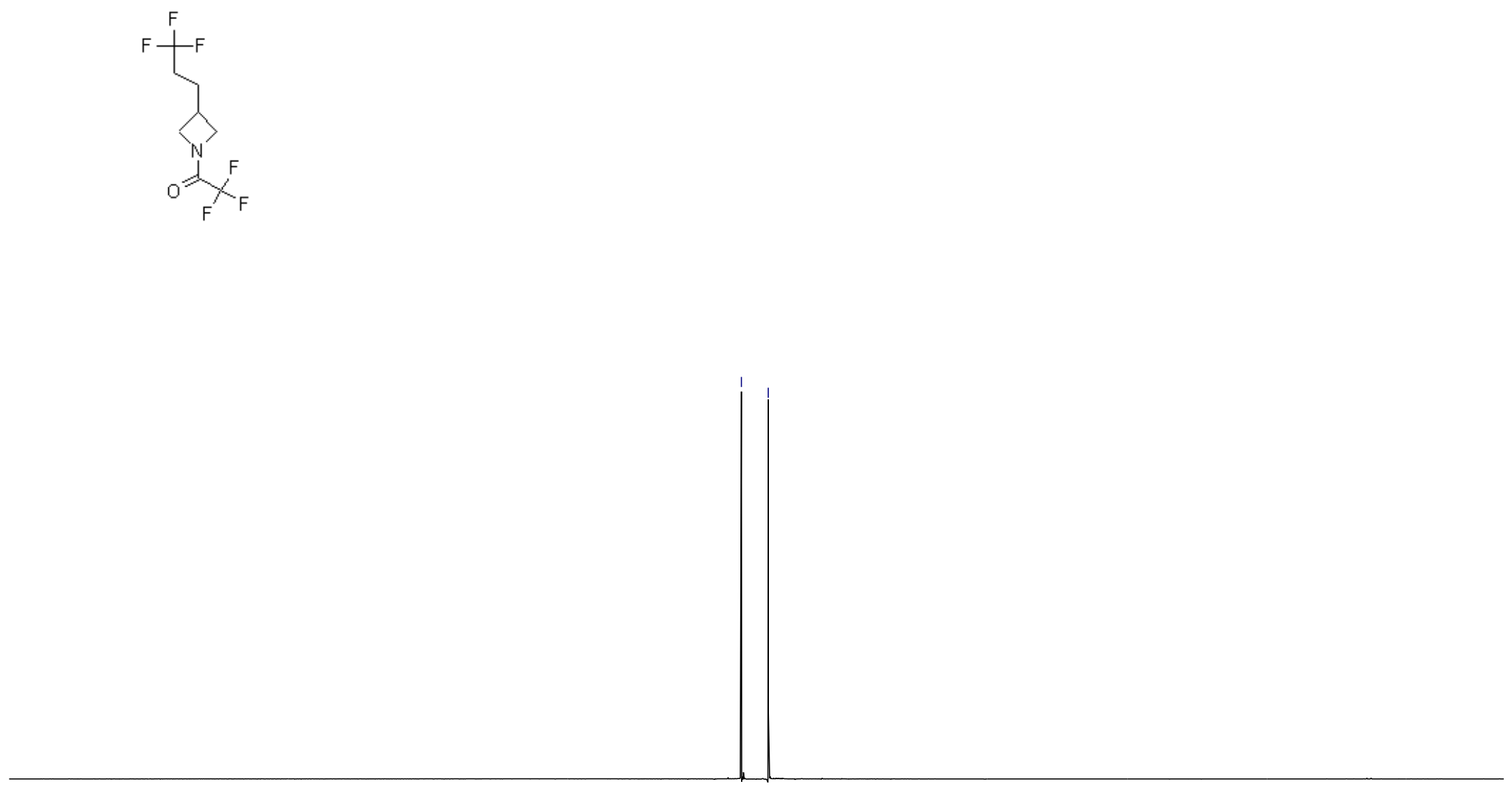

$\begin{array}{llllllllll}110 & 90 & 80 & 70 & 60 & 50 & 40 & 30 & 20 & 10\end{array}$ 


\section{Compound 10a}

mom-5792

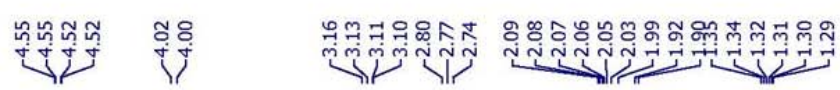

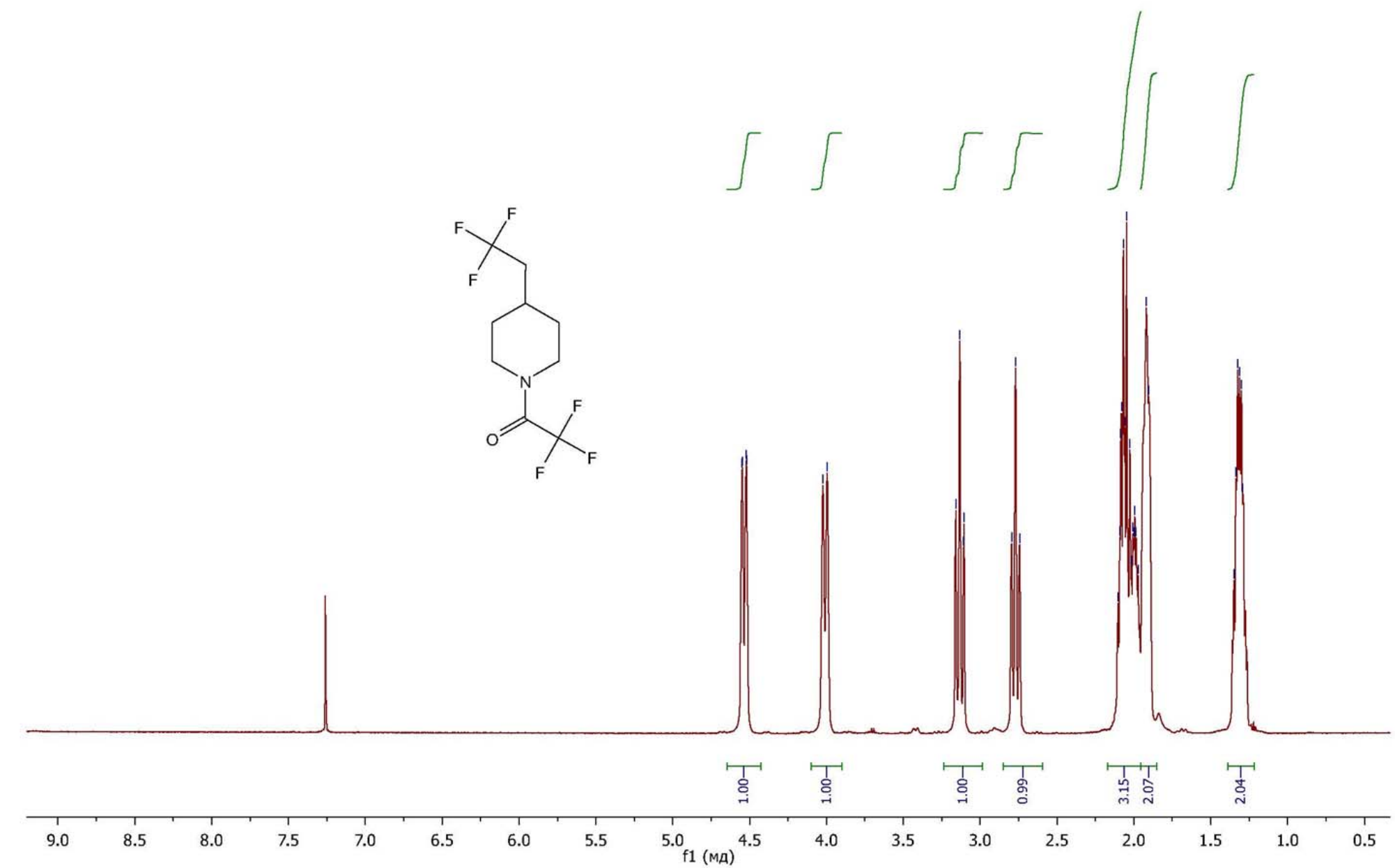




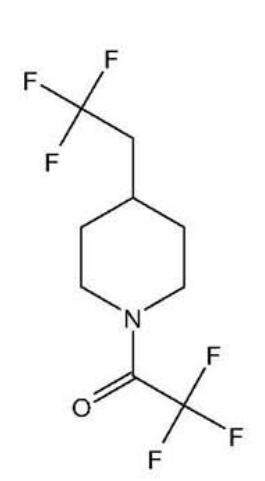

(n) 
mom-5792_F19\{H\}
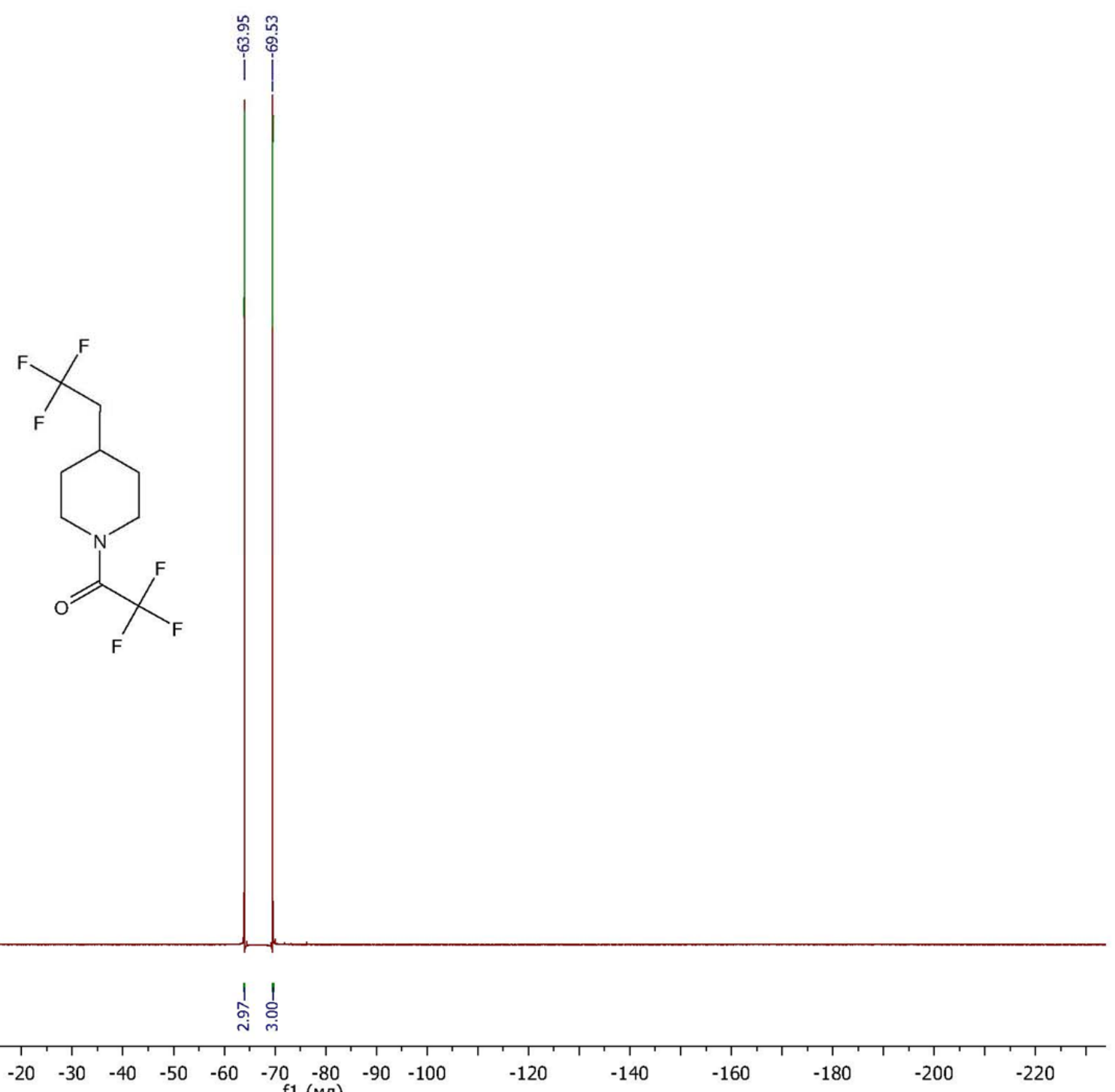


\section{Compound 11a}

tp11777

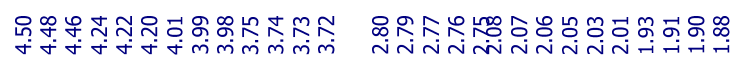

U
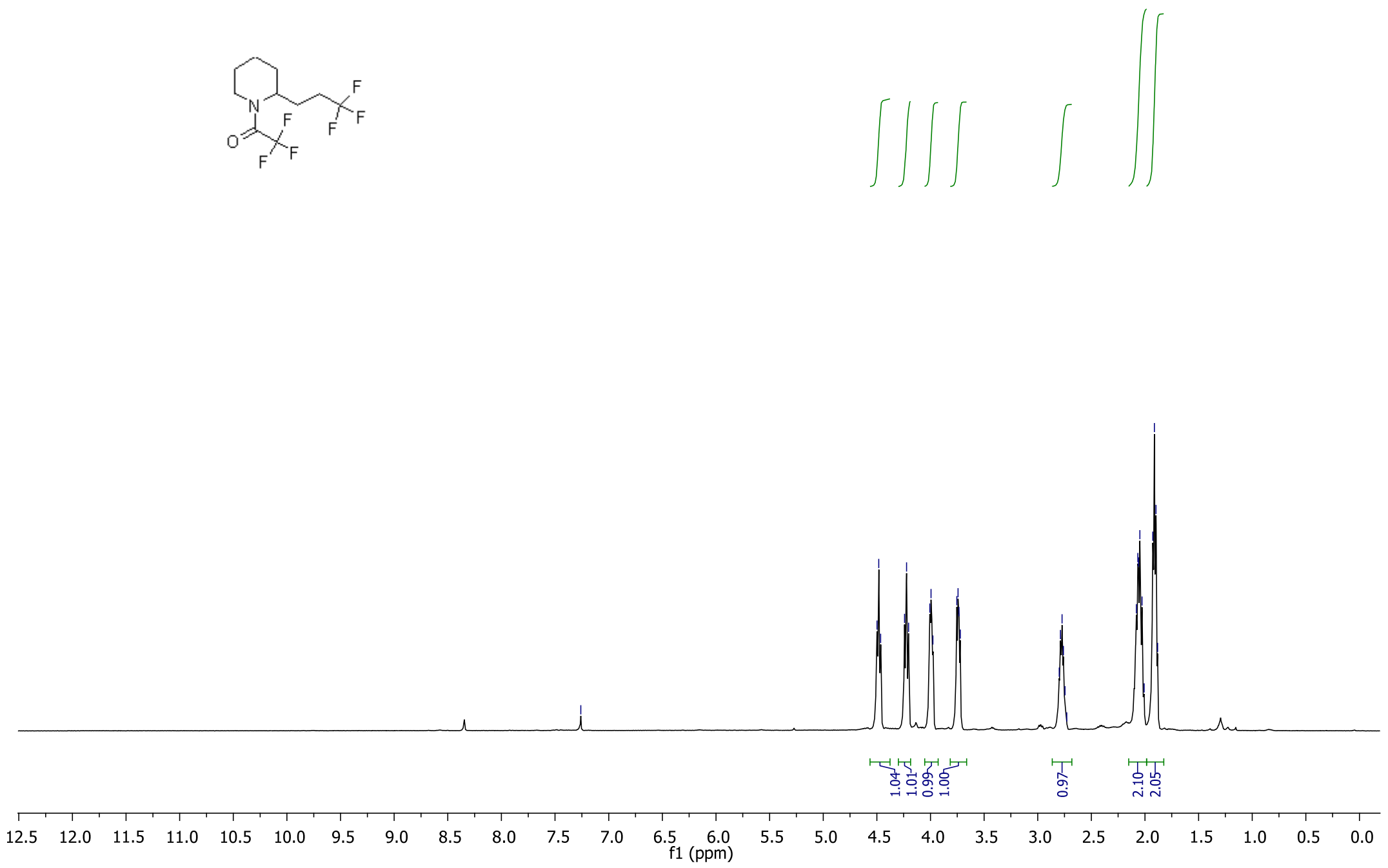


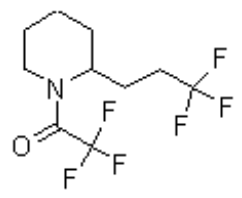

w. W.

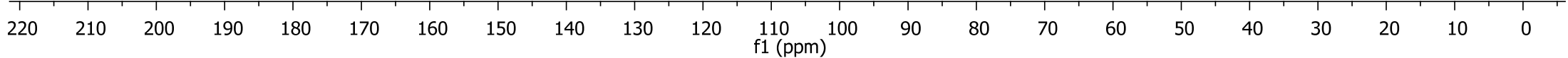


tp11777_F19 $\{\mathrm{H}\}$

$19 \mathrm{~F}-\{1 \mathrm{H}\}$

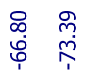

${ }_{{ }_{F}}{ }_{F}{ }_{F}^{F}$

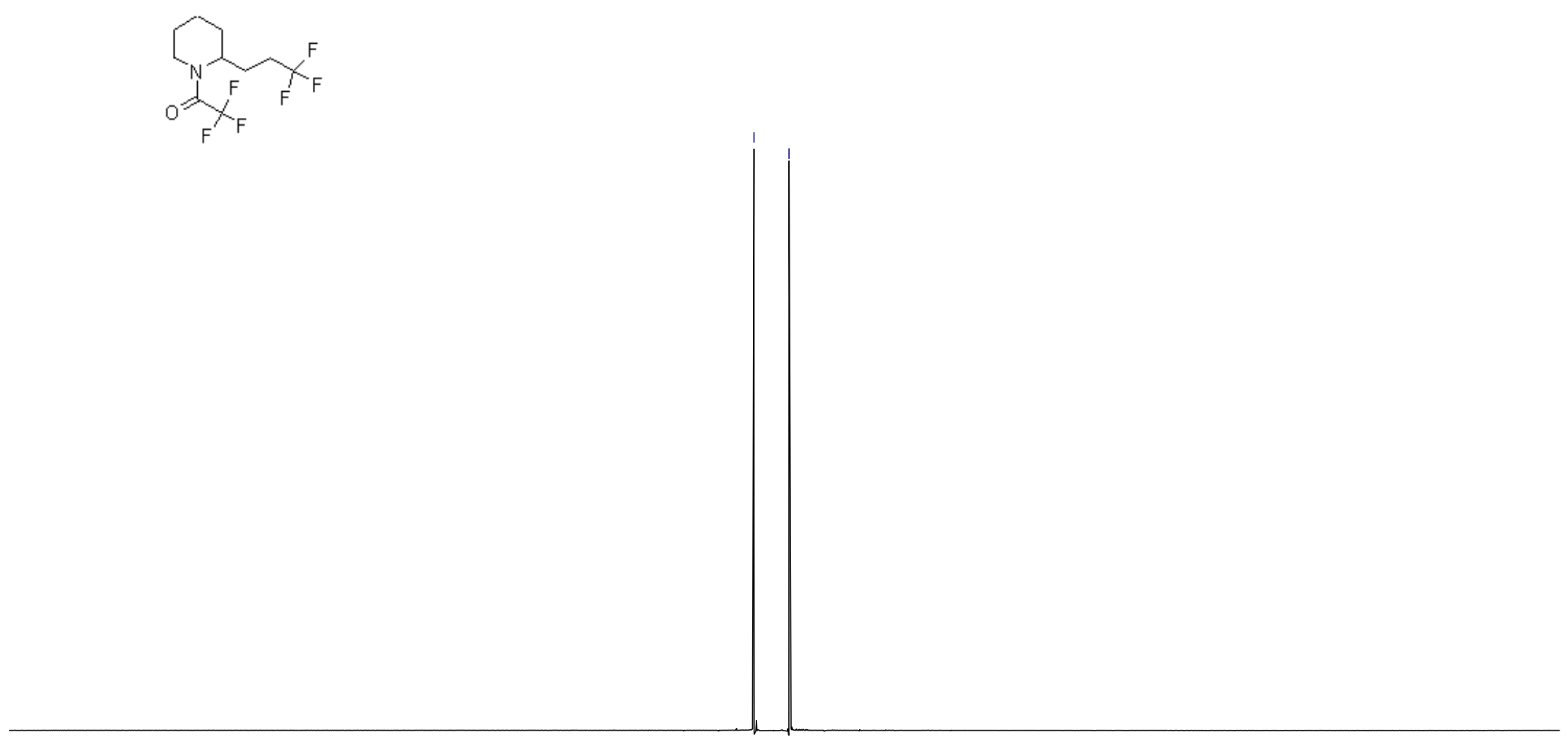

$\begin{array}{llllllllllllllllll}70 & 60 & 50 & 40 & 30 & 20 & 10 & 0 & -10 & -20 & -30 & -40 & -50 & -60 & -70 & -80 & -90 & -100\end{array}$ 


\section{Compound 12a}

R519634

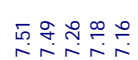

$\underbrace{\stackrel{m}{m} m^{m}}_{m}$

रîn

$\mathrm{CF}_{3}$
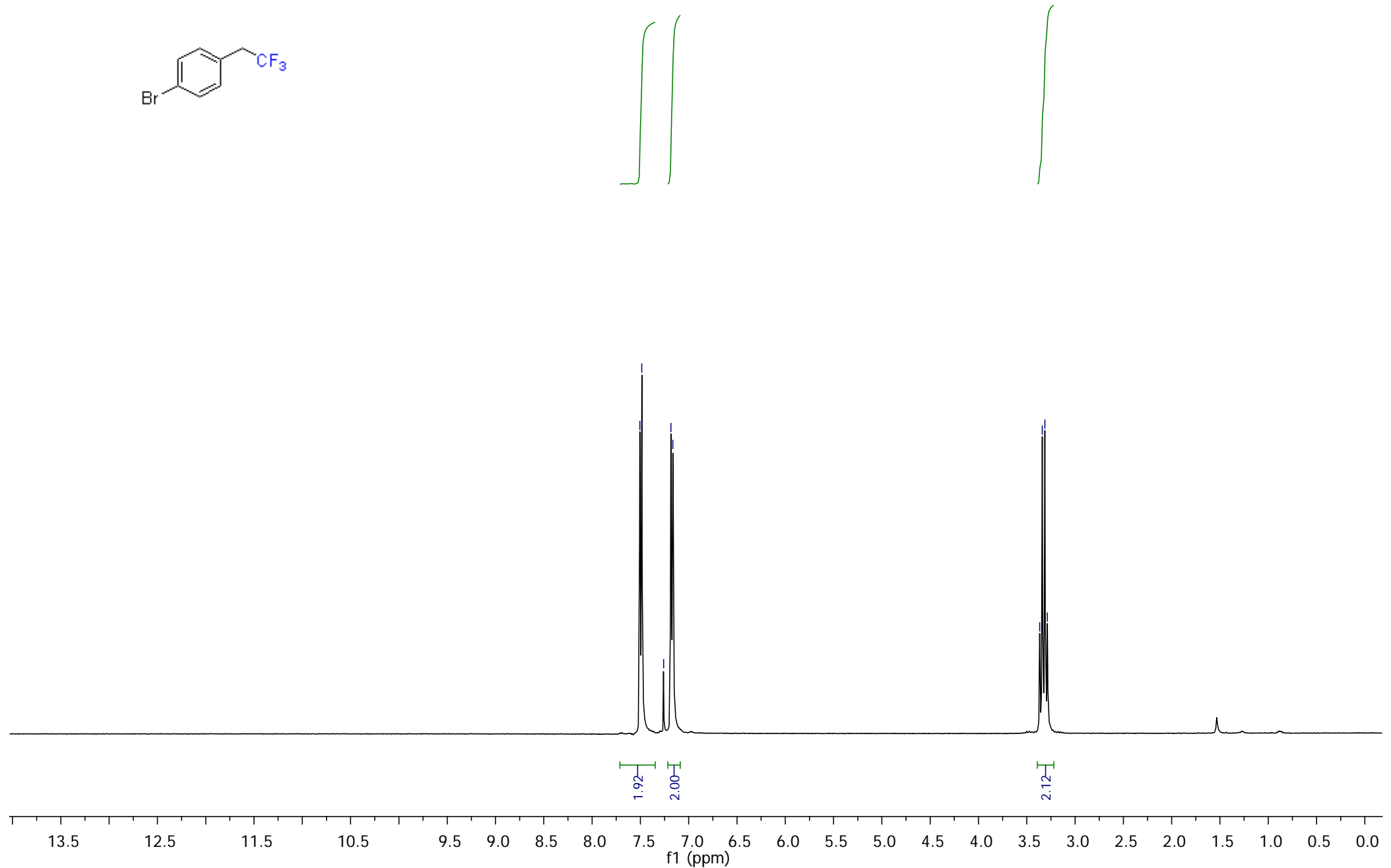
R519634_C13

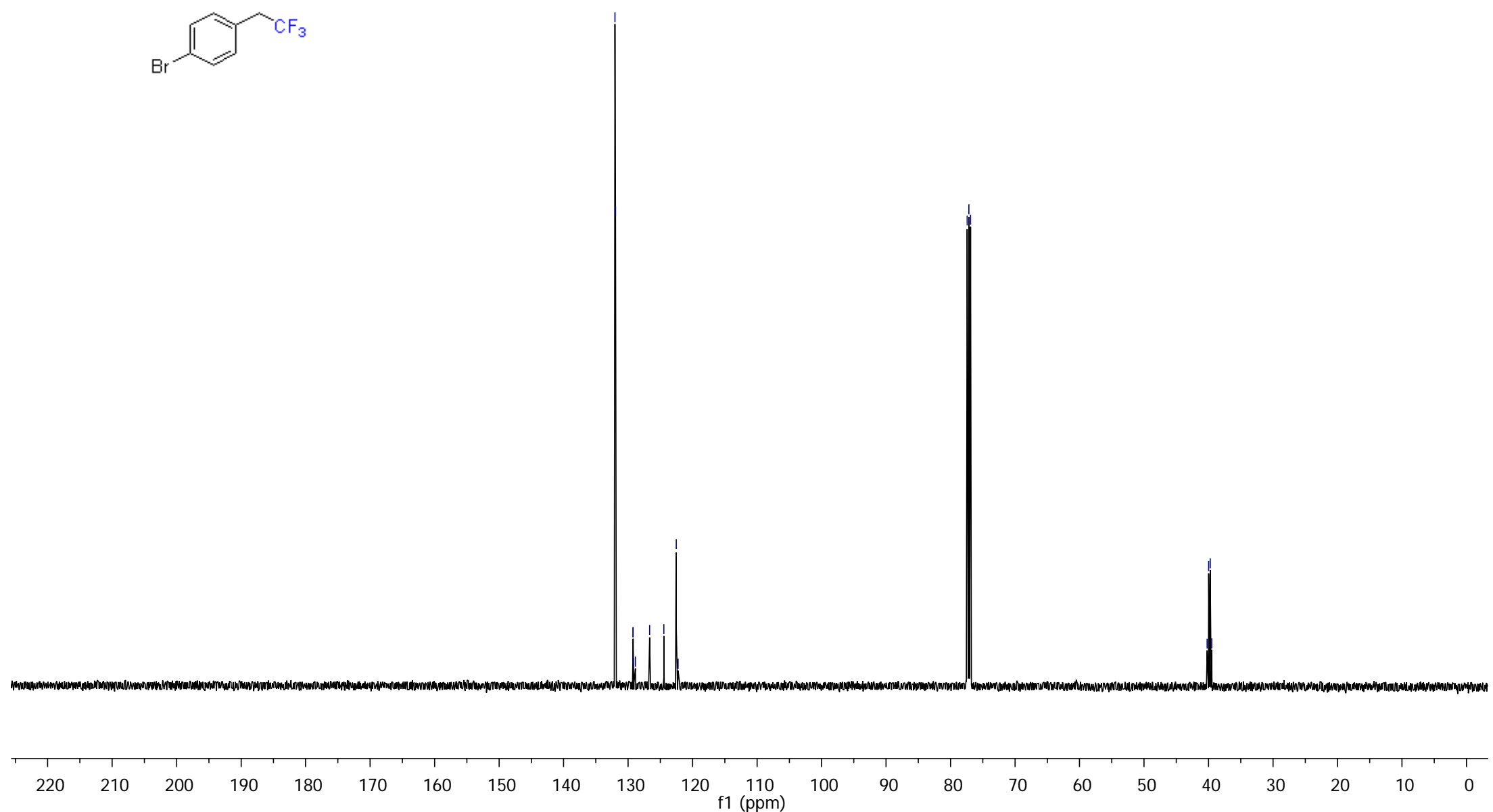


R519634_F19 $\{\mathrm{H}\}$ $19 F-\{1 \mathrm{H}\}$

$\mathrm{CF}_{3}$

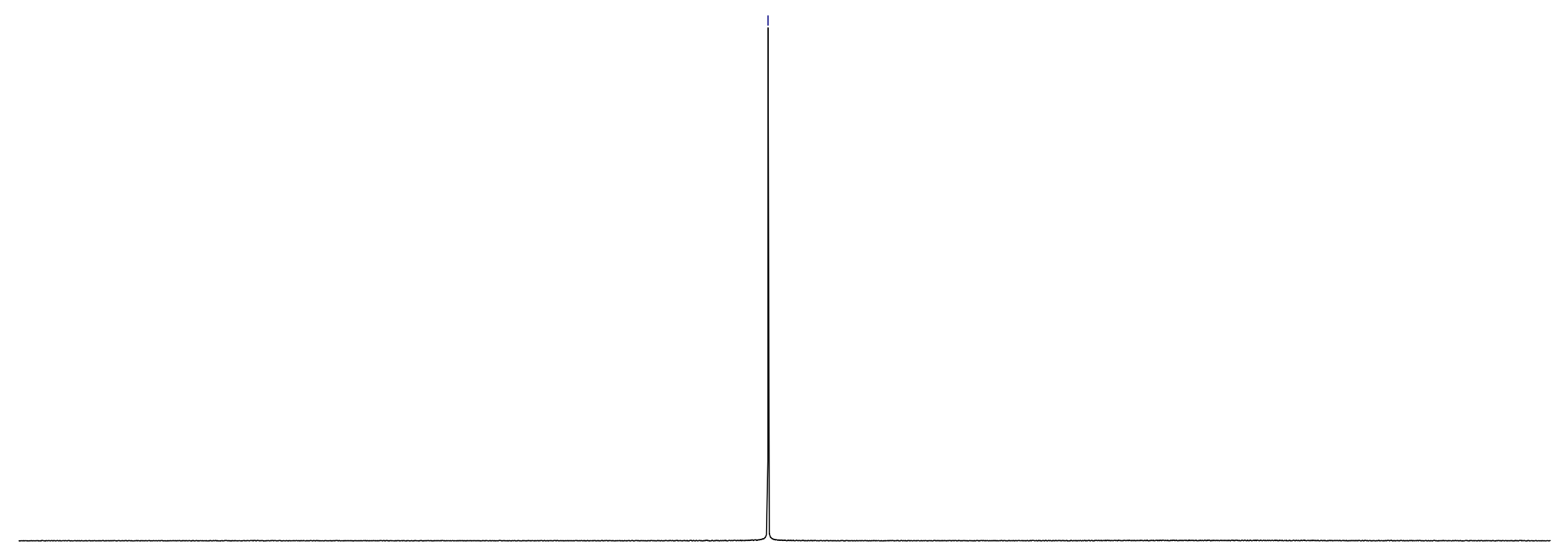




\section{Compound 13a}

R519652

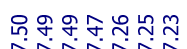

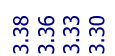

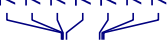

$\underbrace{m i n j m}$

$\overbrace{\mathrm{Br}} \mathrm{CF}_{3}$
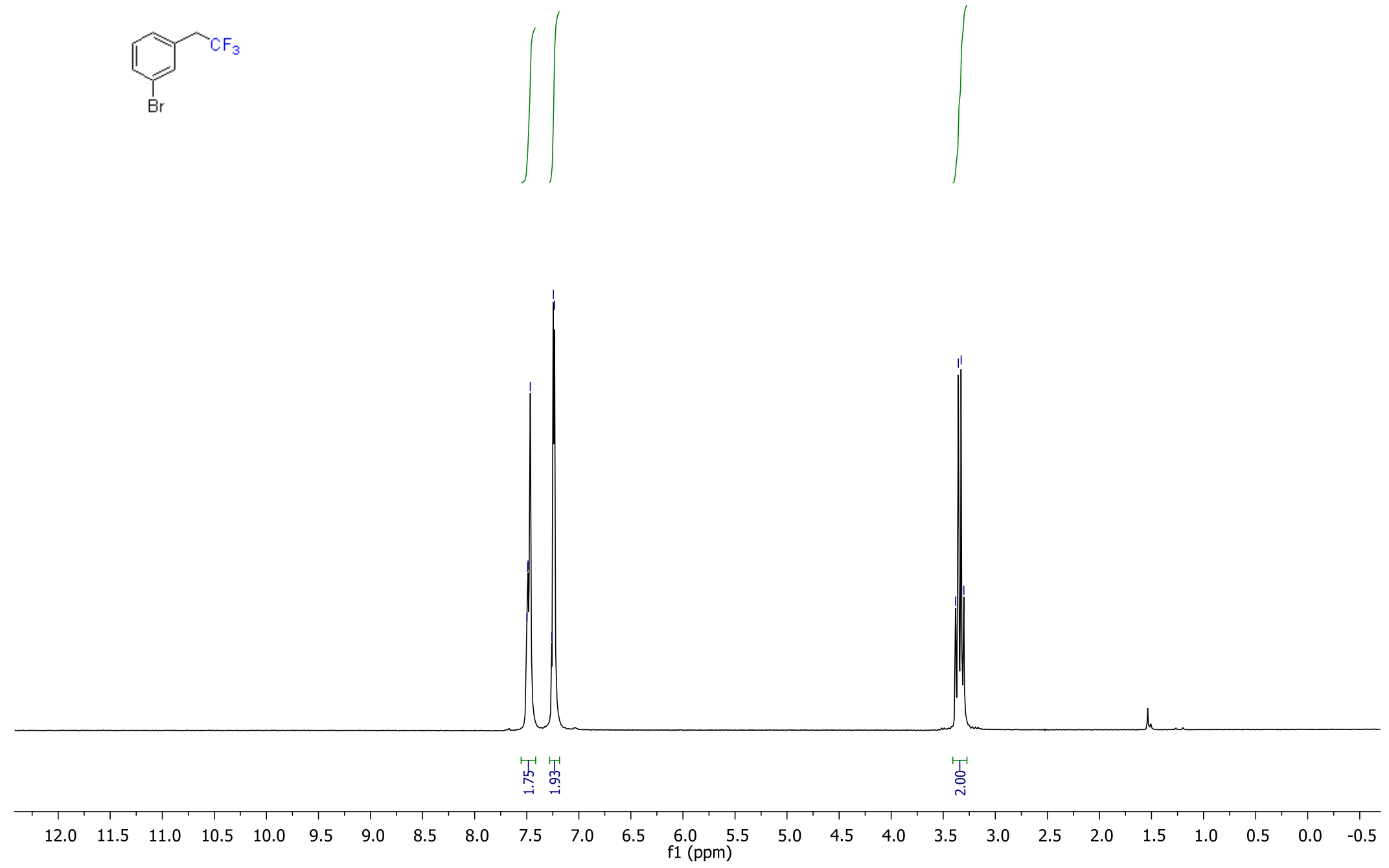

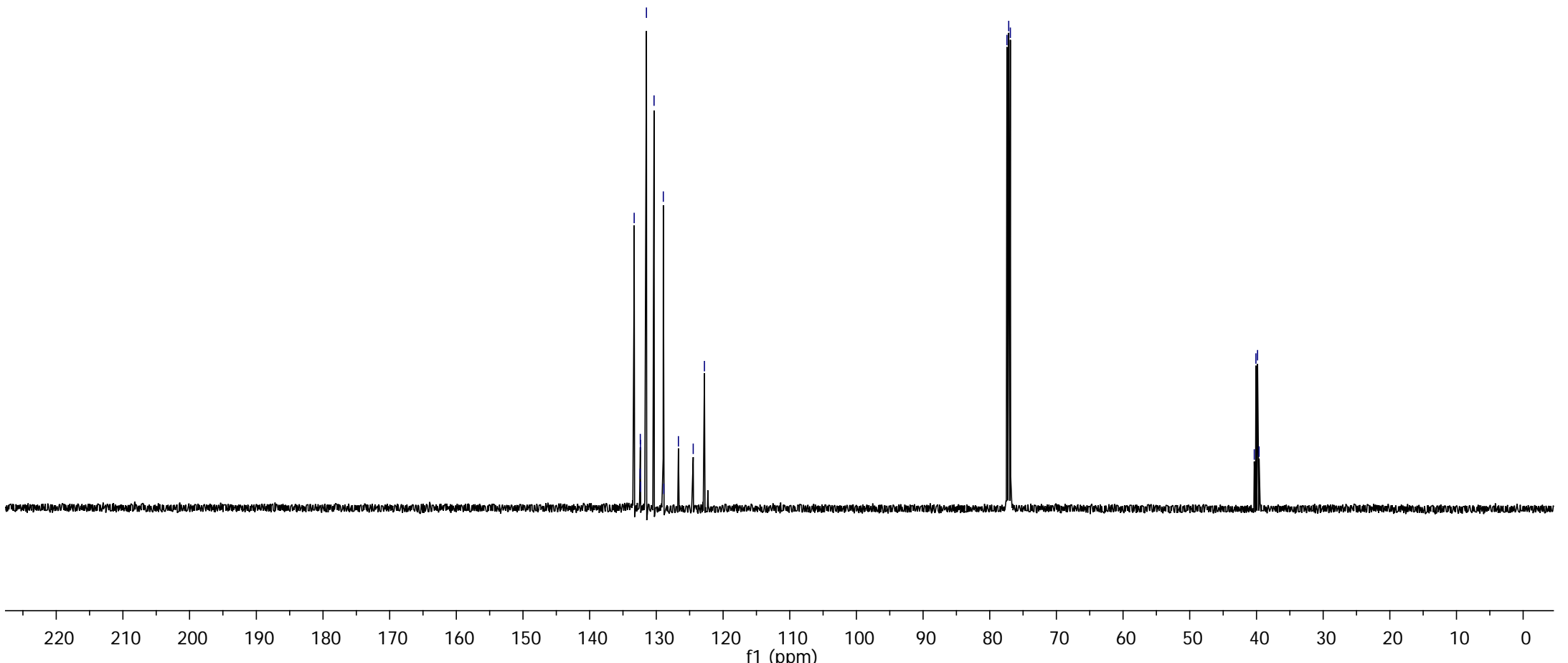
R519652_F19 $\{\mathrm{H}\}$

$19 \mathrm{~F}-\{1 \mathrm{H}\}$

$\overbrace{\mathrm{Br}} \mathrm{CF}_{3}$

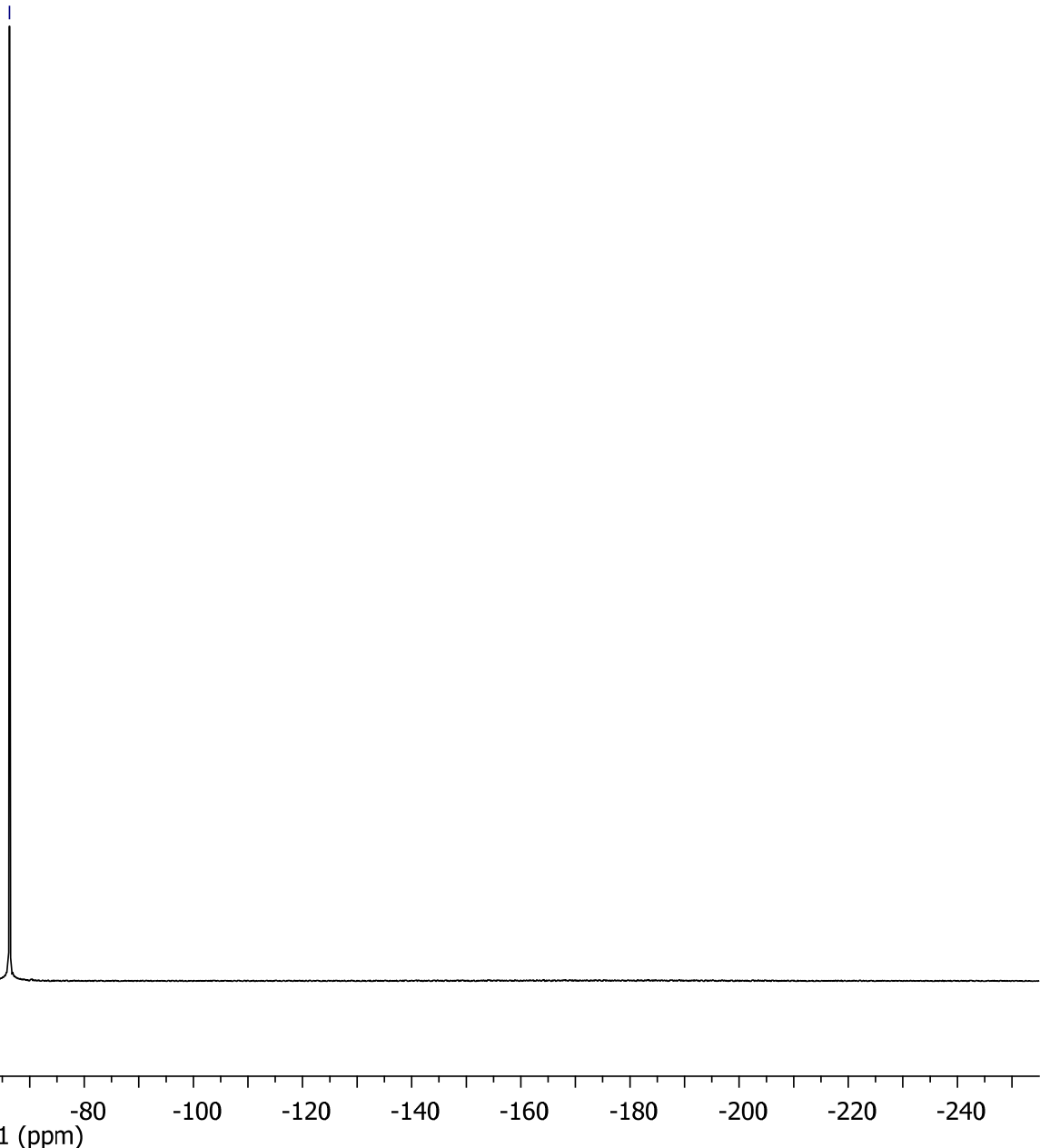




\section{Compound 14a}

R519636

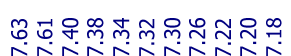

닌.

电皆
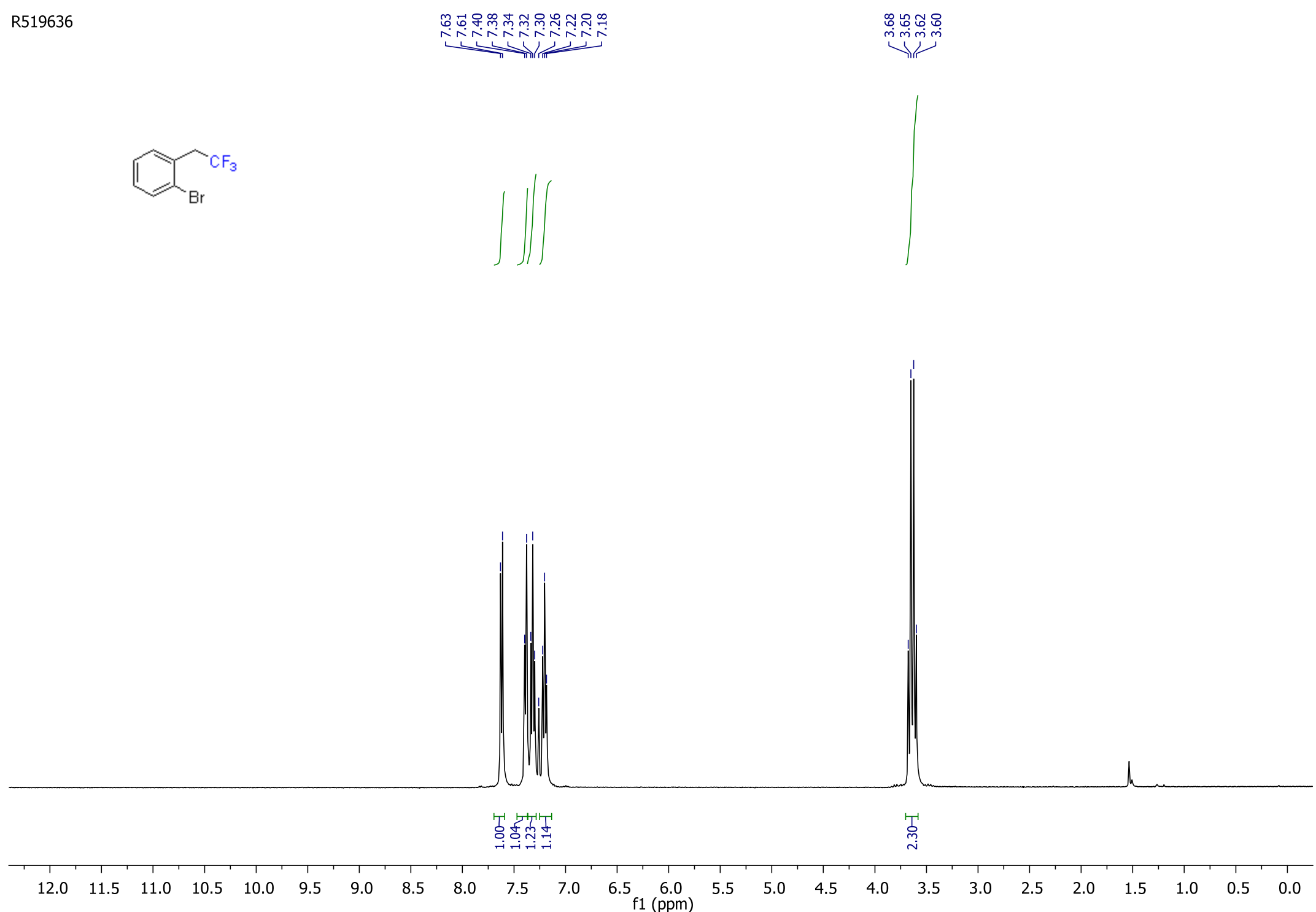
$1 \mathrm{Br}_{\mathrm{Br}} \mathrm{CF}_{3}$

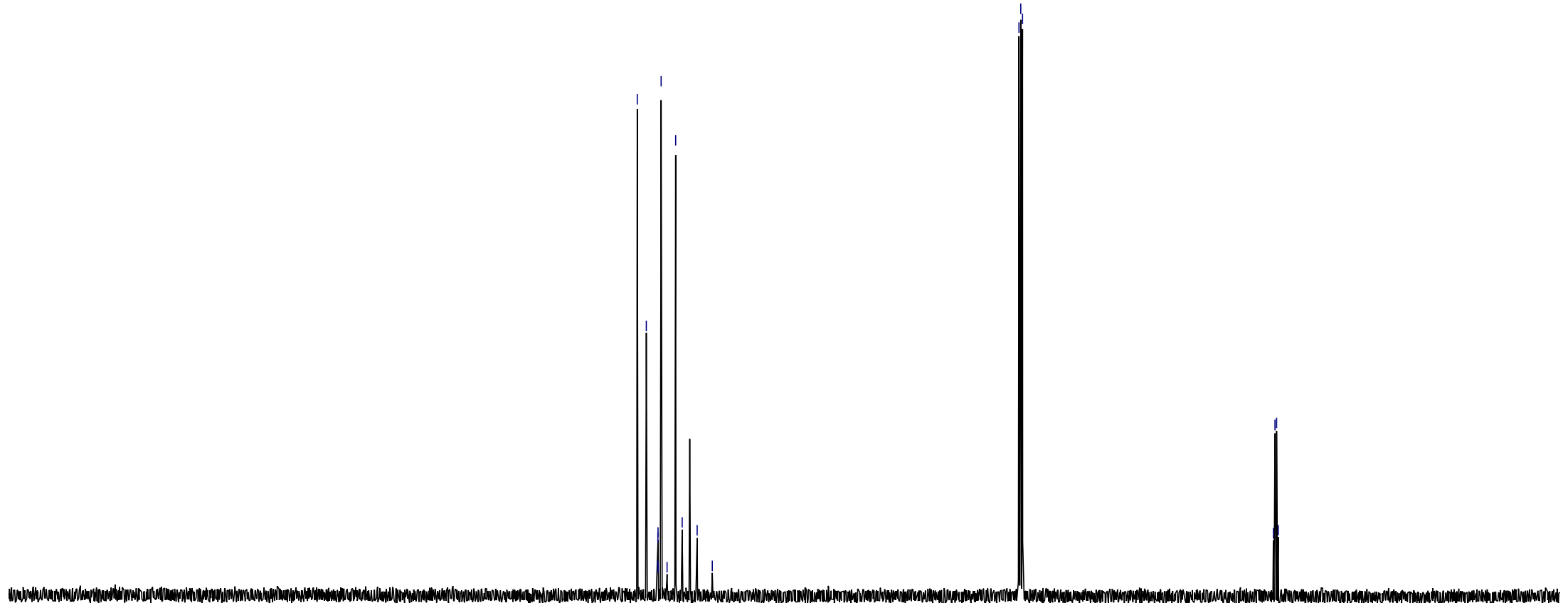

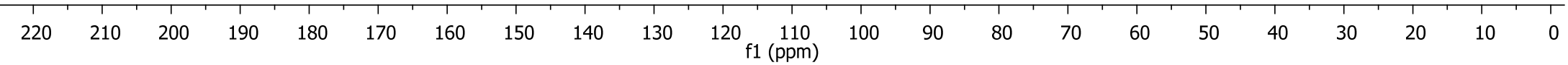


R519636_F19 $\{\mathrm{H}\}$

$\overbrace{\mathrm{Br}} \mathrm{CF}_{3}$
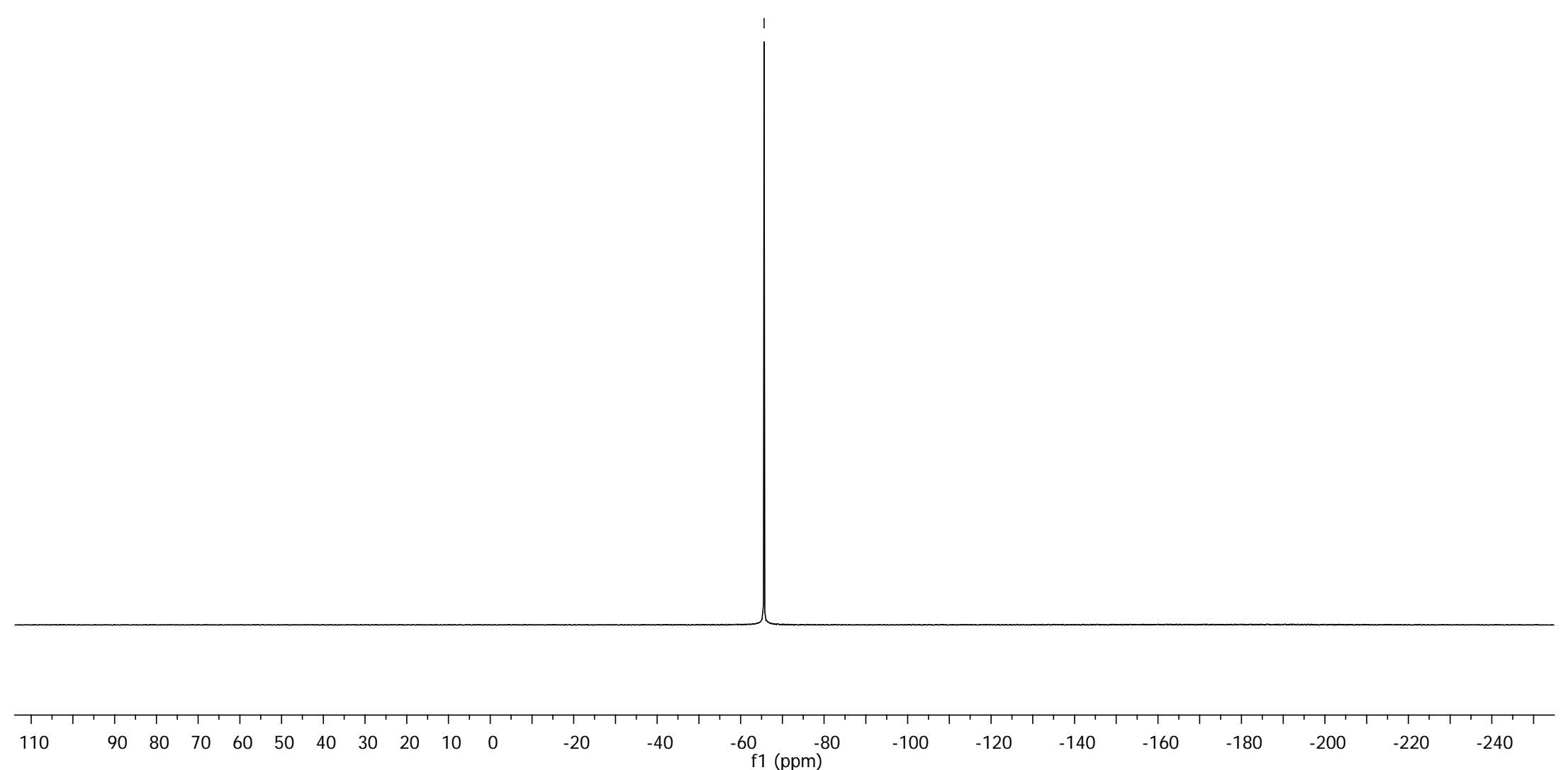
Compound 15a

촐

UOtp-1912

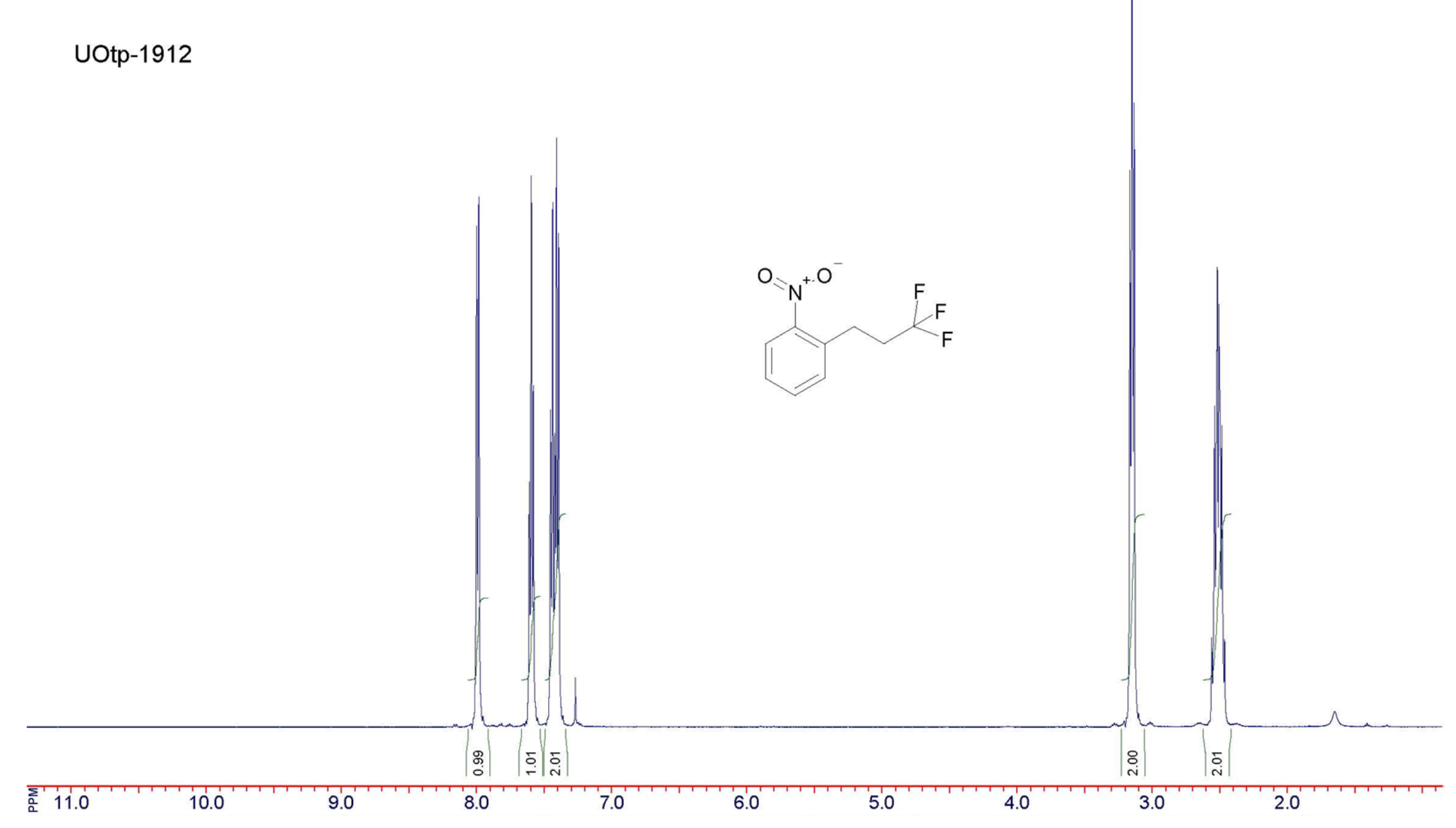

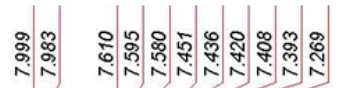

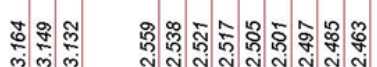

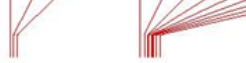




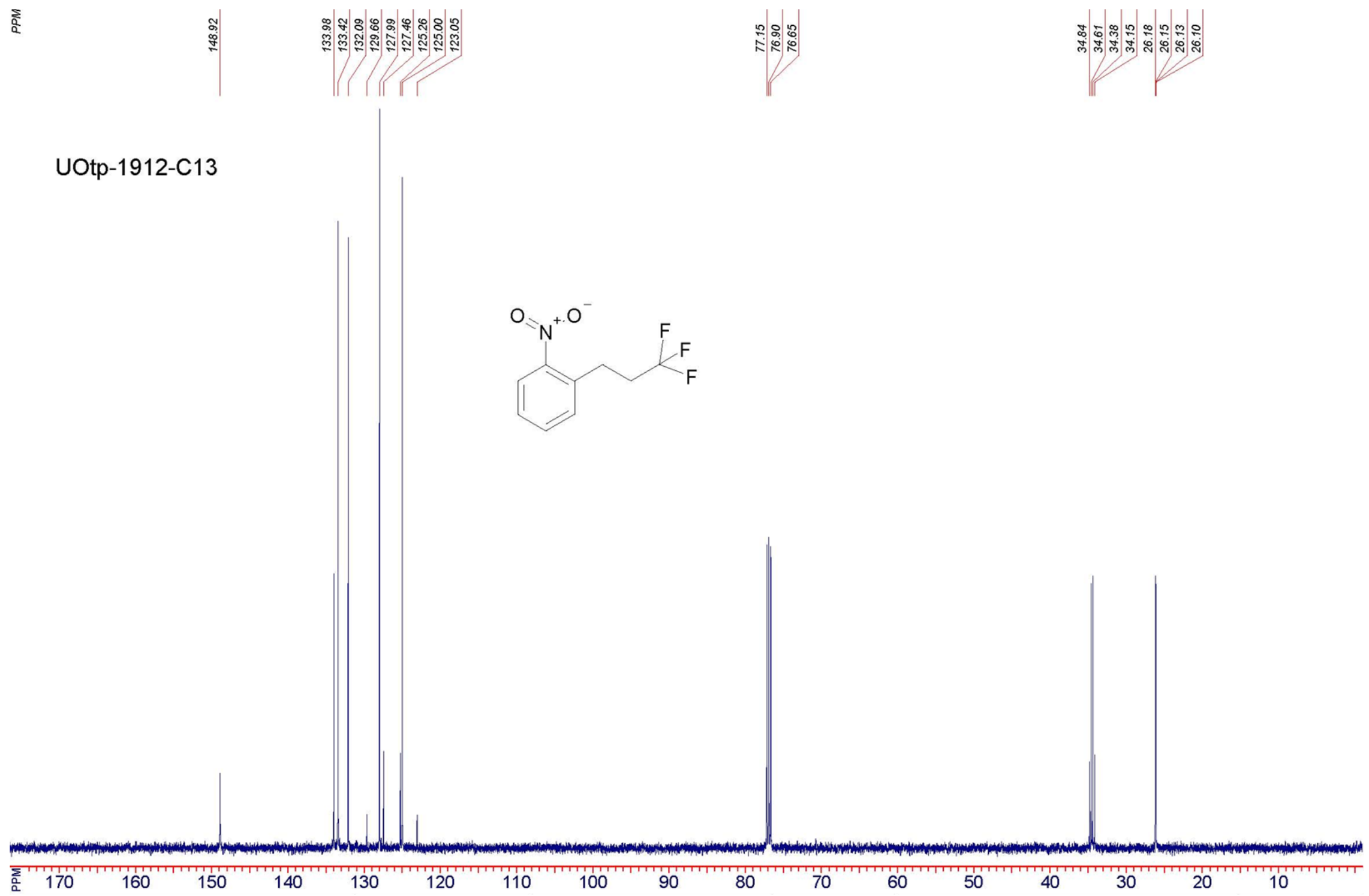




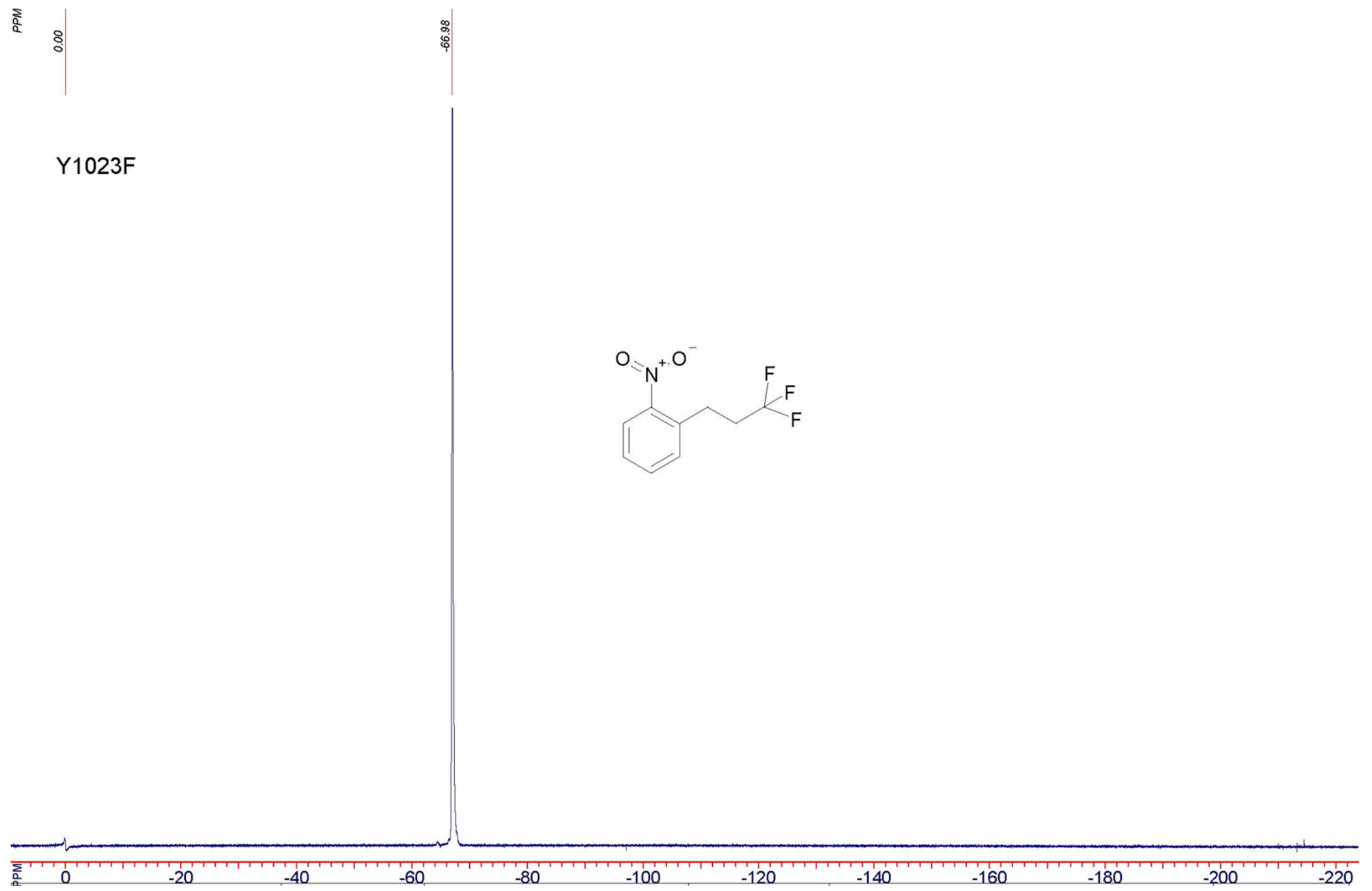




\section{Compound 16a}

UOtp-1916

웅

$\underbrace{2}$

我垗

$\mathrm{CF}_{3}$
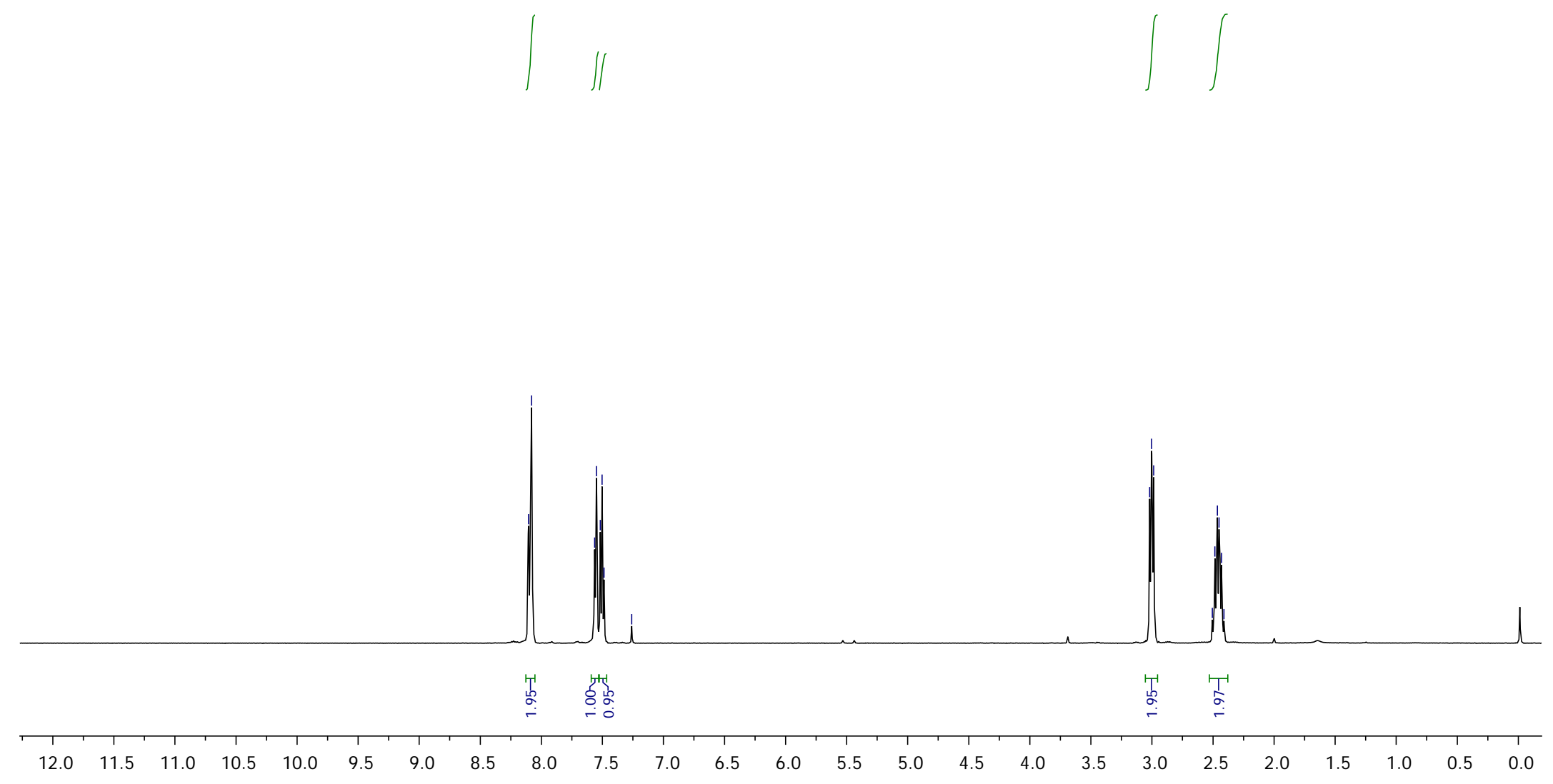
UOtp-1916-C13

กู่

OC

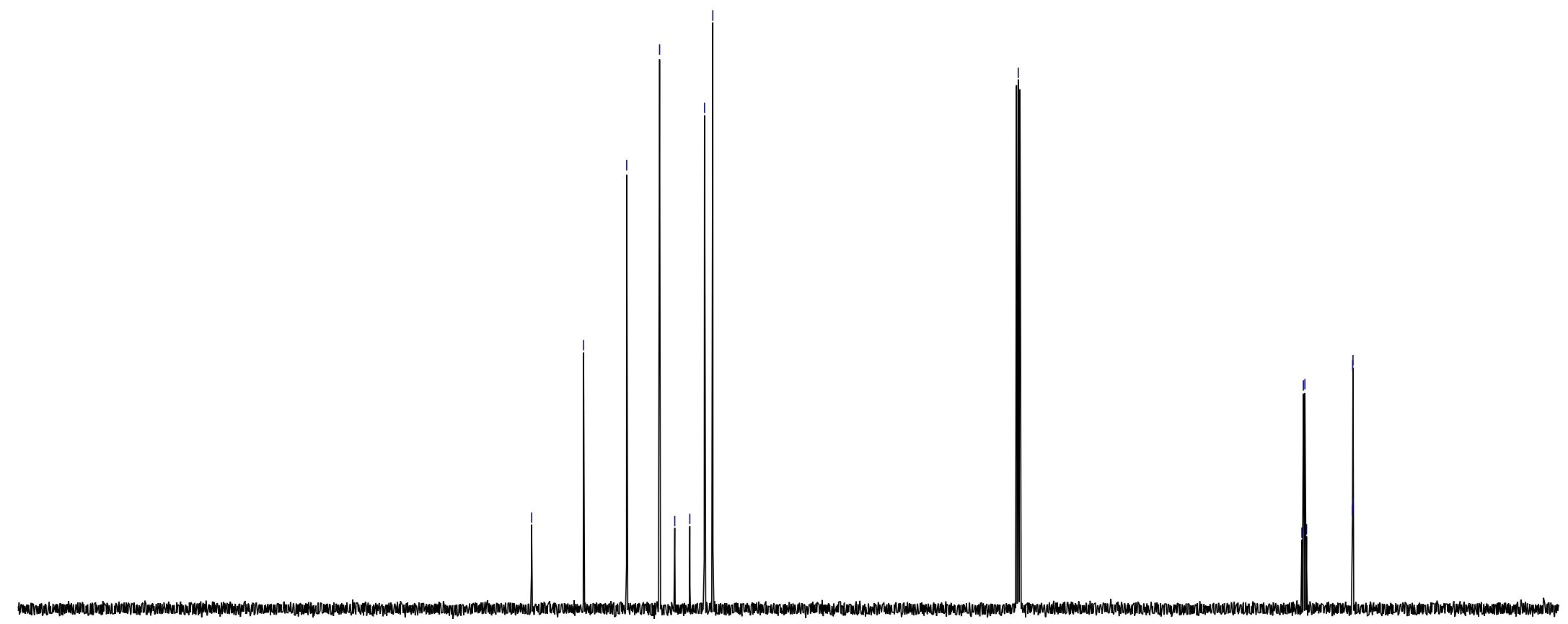

$\begin{array}{lllllllllllll}220 & 210 & 200 & 190 & 180 & 170 & 160 & 150 & 140 & 130 & 120 & 110 & 100\end{array}$

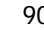

80

60

40

30

20 $10 \quad 0$ 


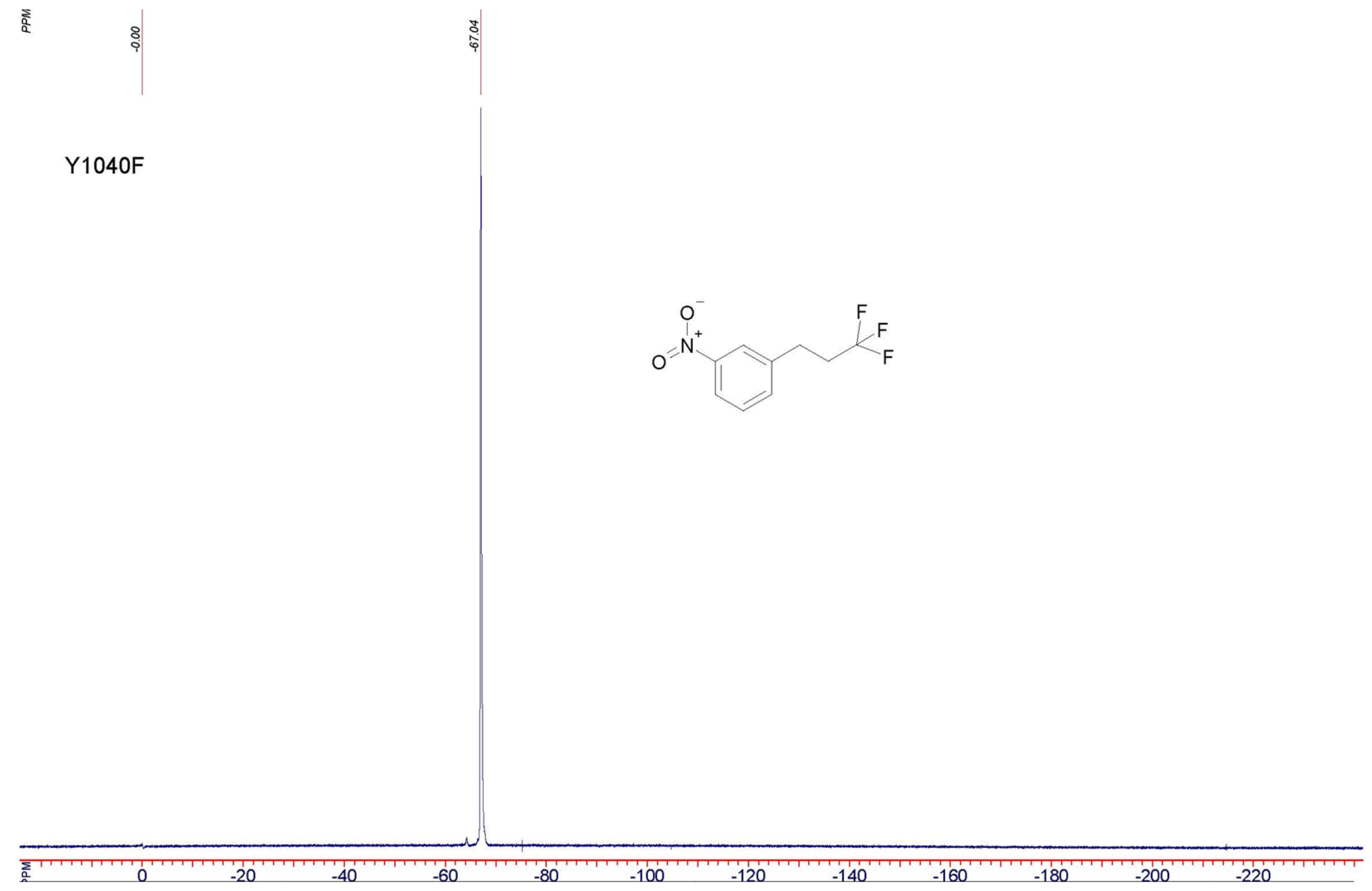




\section{Compound 17a}

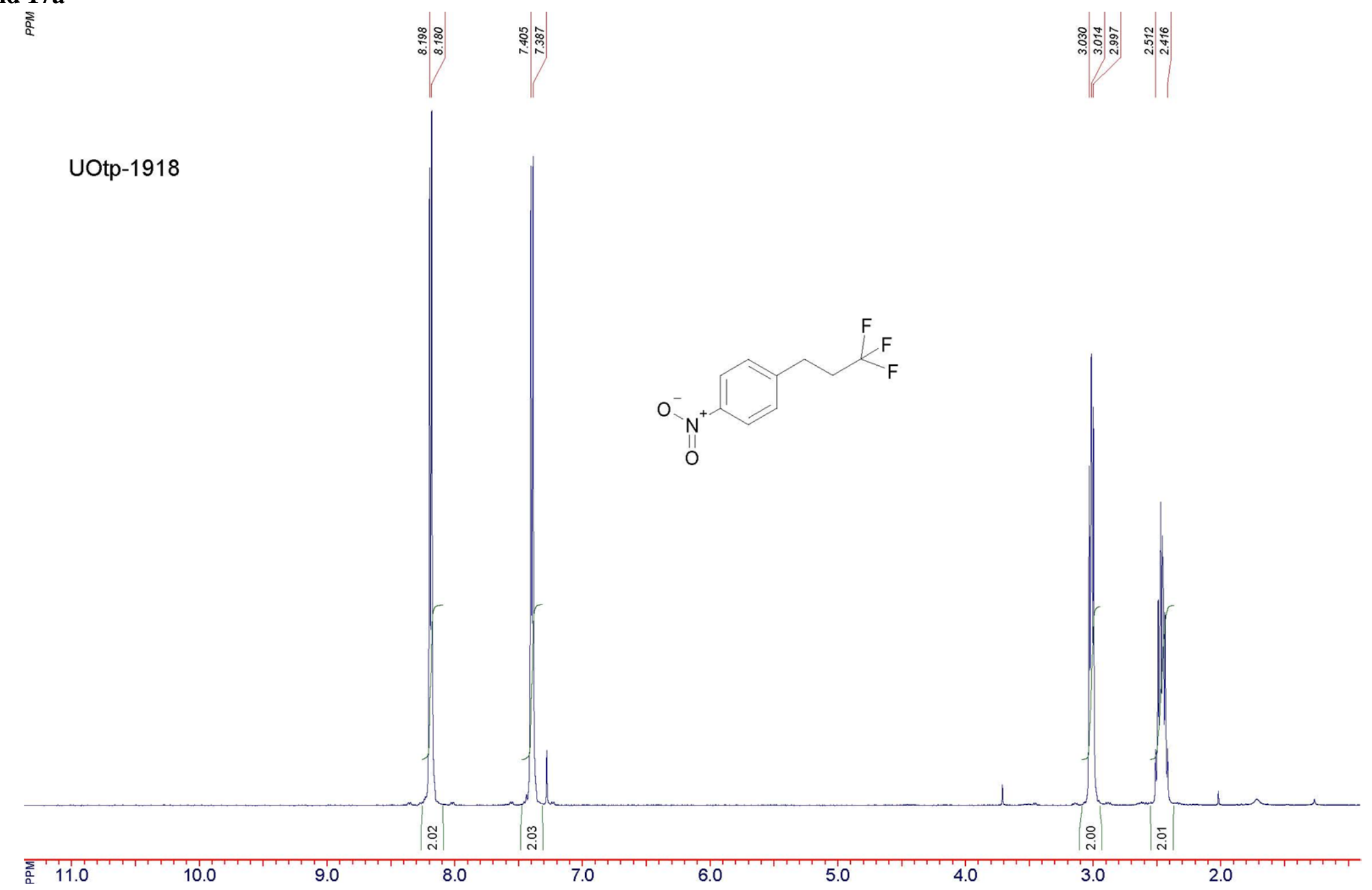




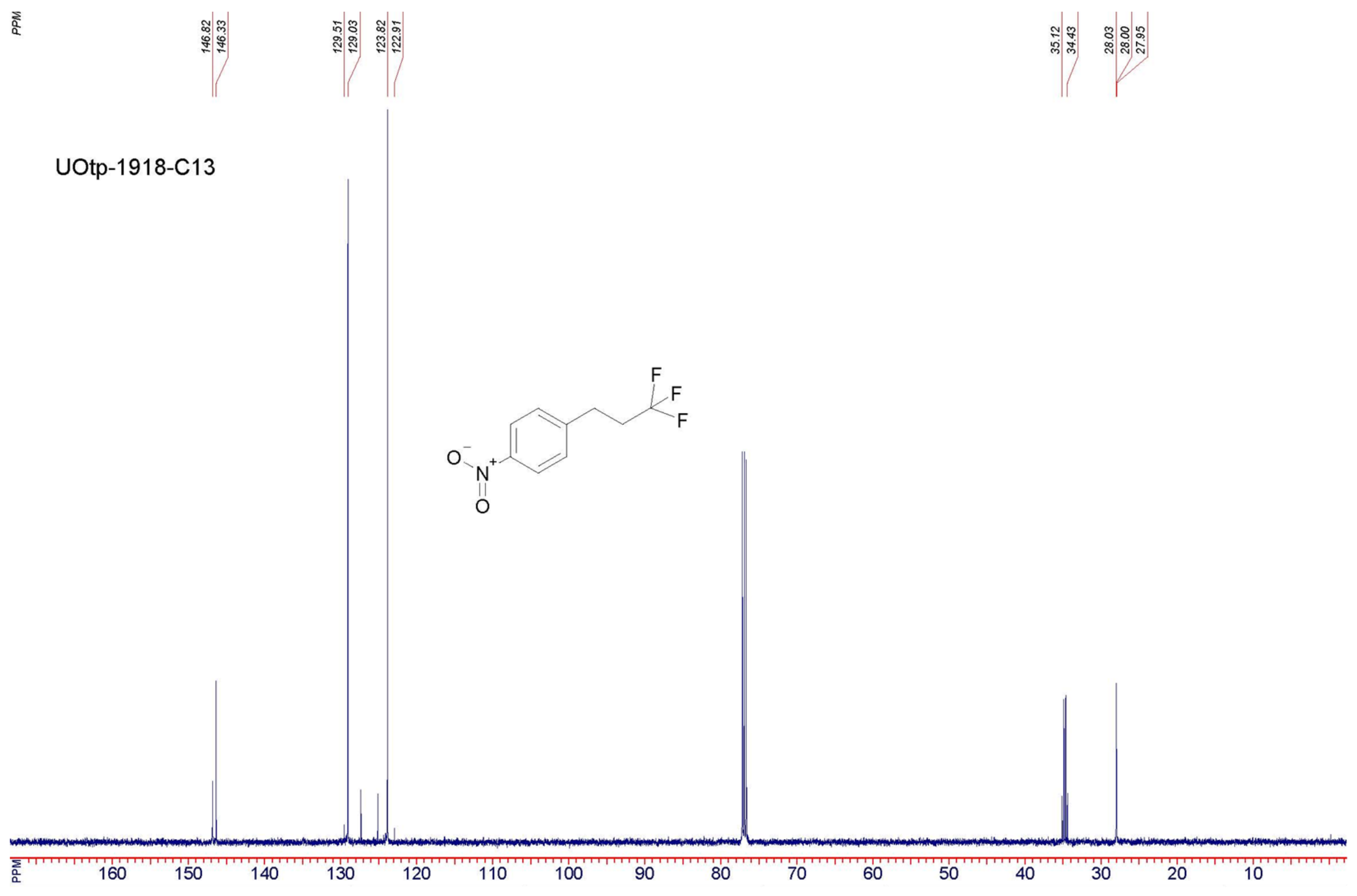




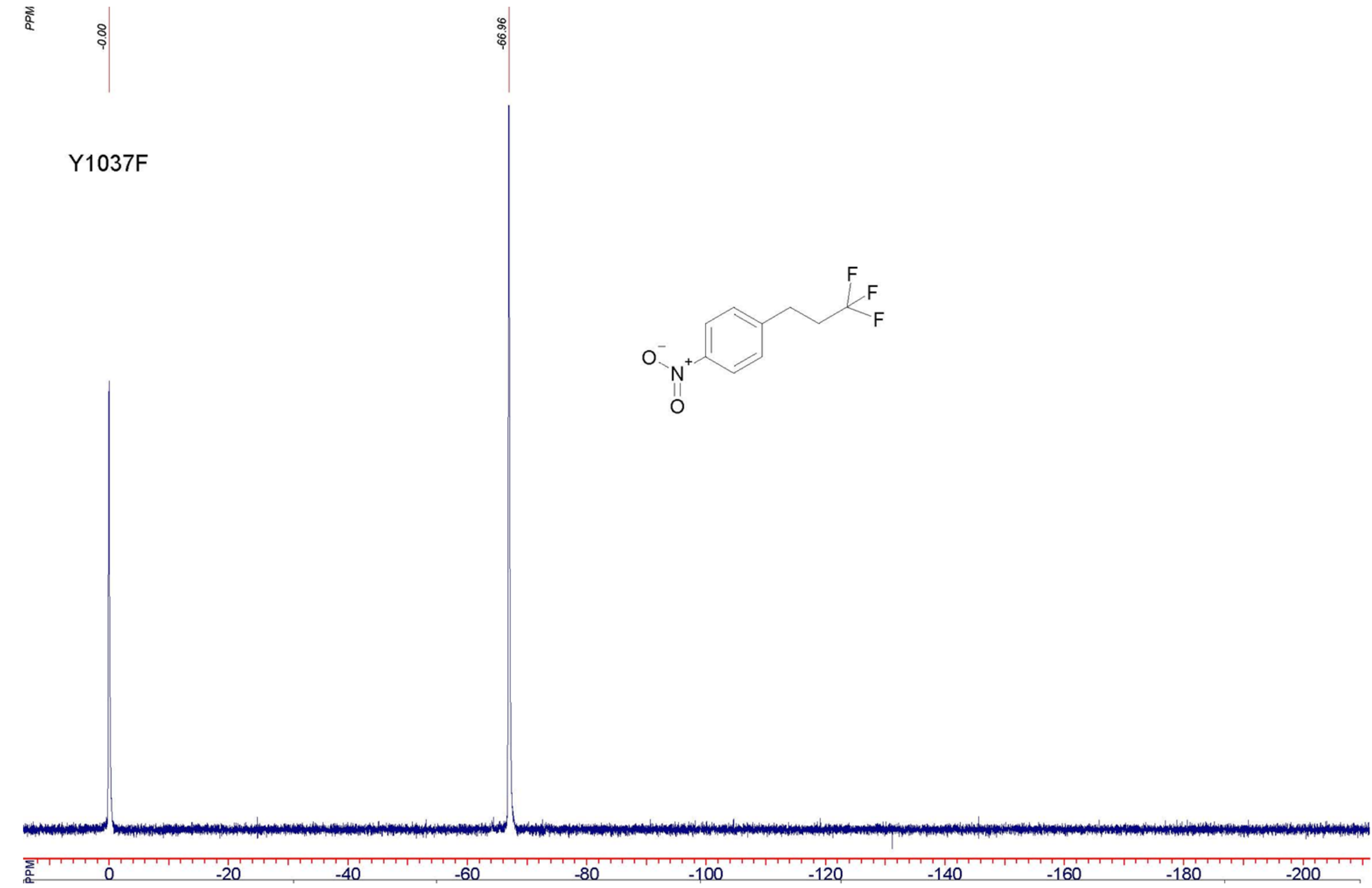




\section{Compound 18a}

R793286

$\mathrm{Br} \curvearrowright \mathrm{CF}_{3}$

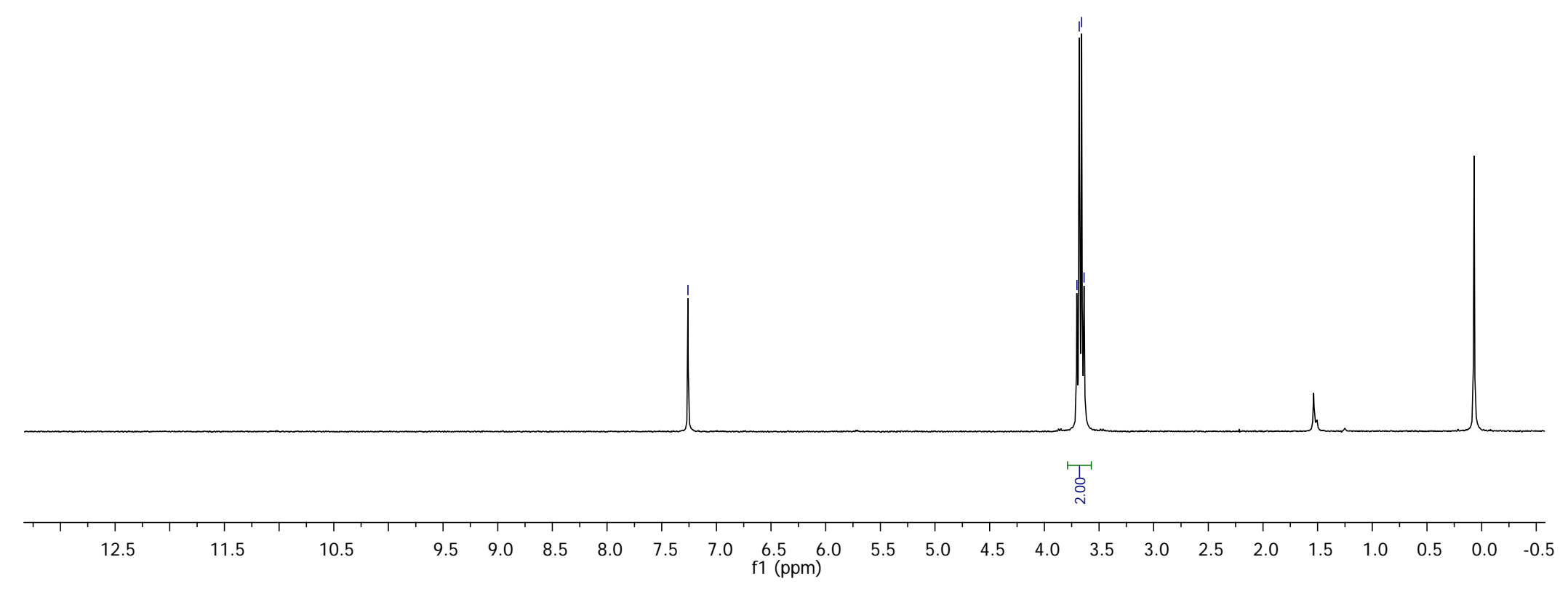


$\mathrm{Br} \curvearrowright \mathrm{CF}_{3}$

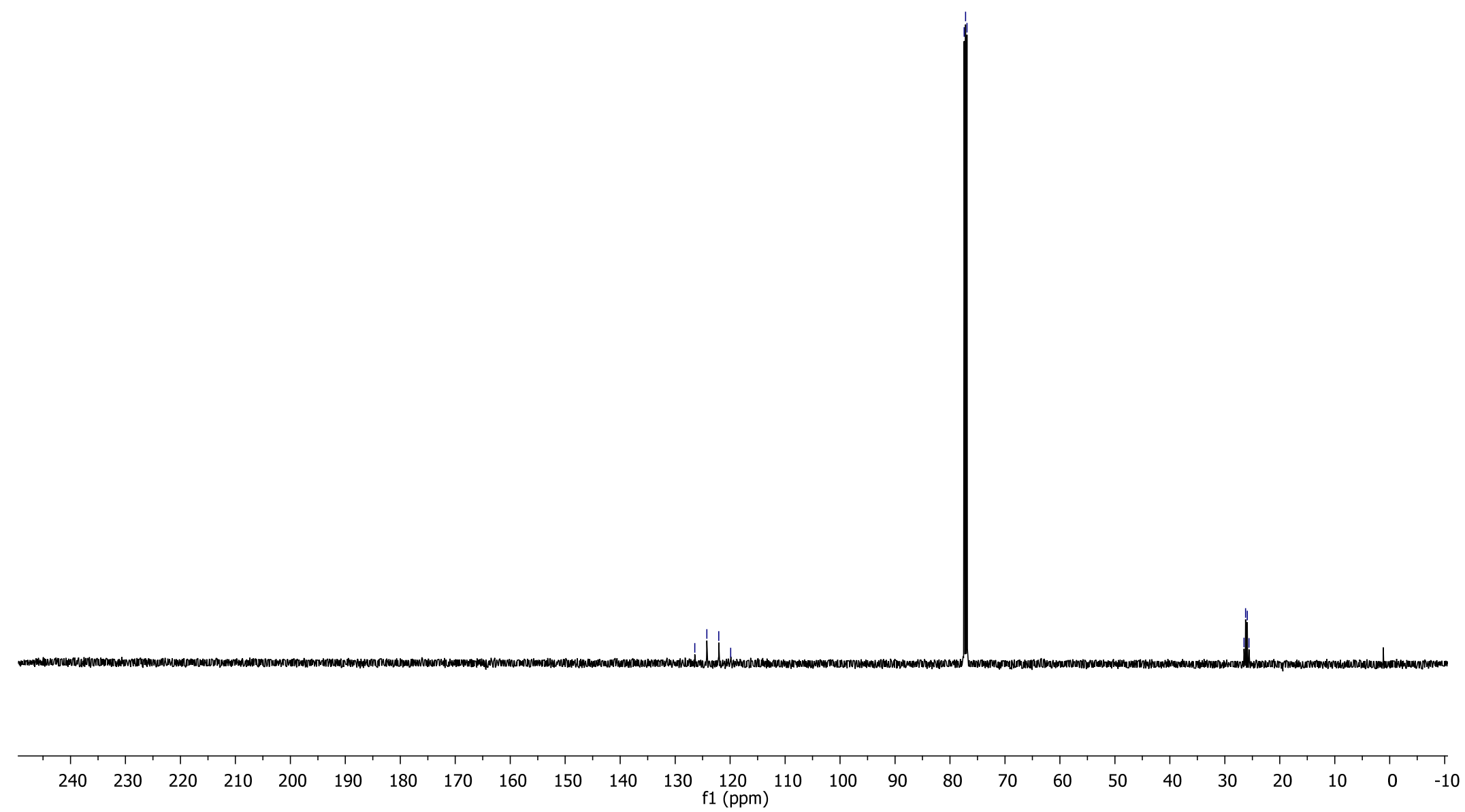


R791974_F19\{H\}

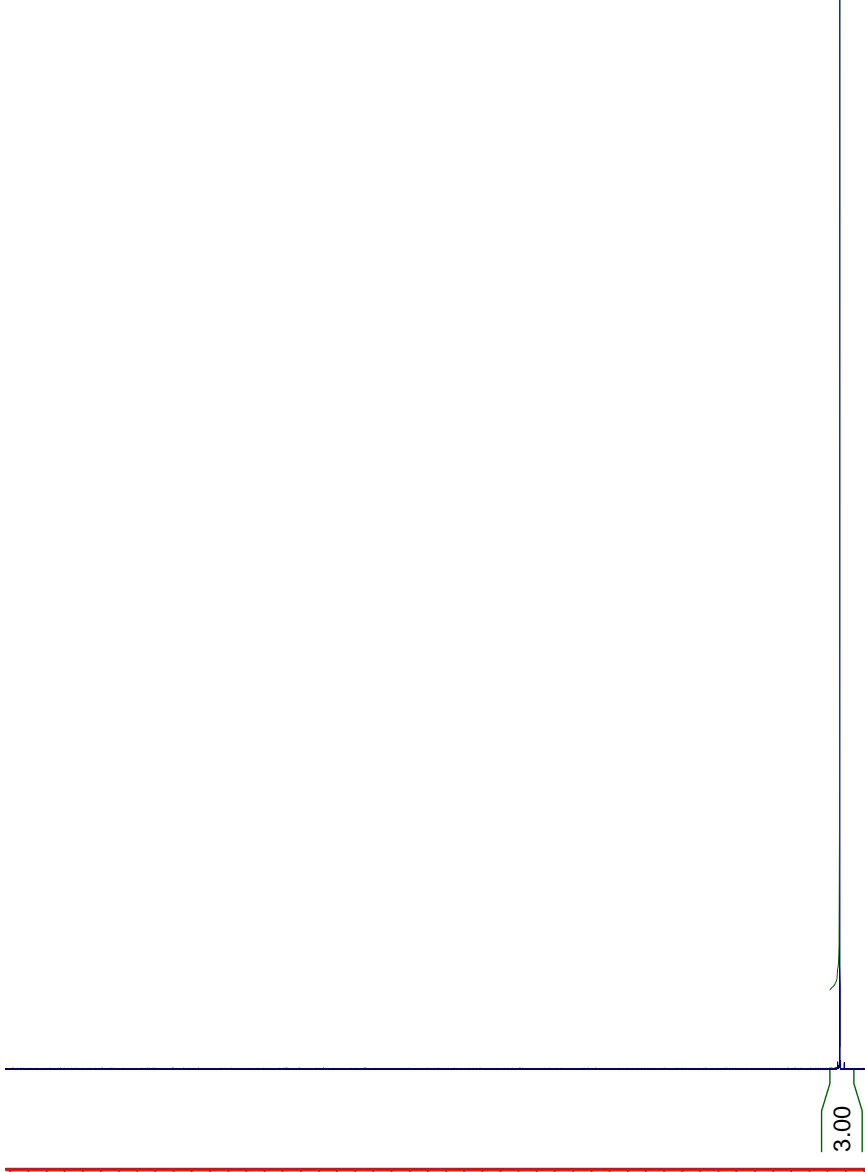

a)

品

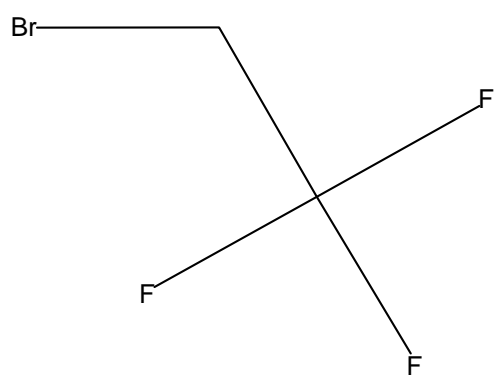

R791974_F19\{H\} C2H2BrF3 162.94

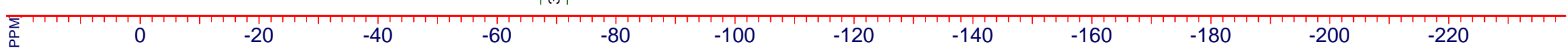




\section{Compound 19a}

R1123829

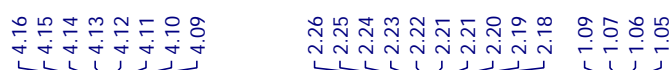

$\overbrace{\mathrm{Br}}$

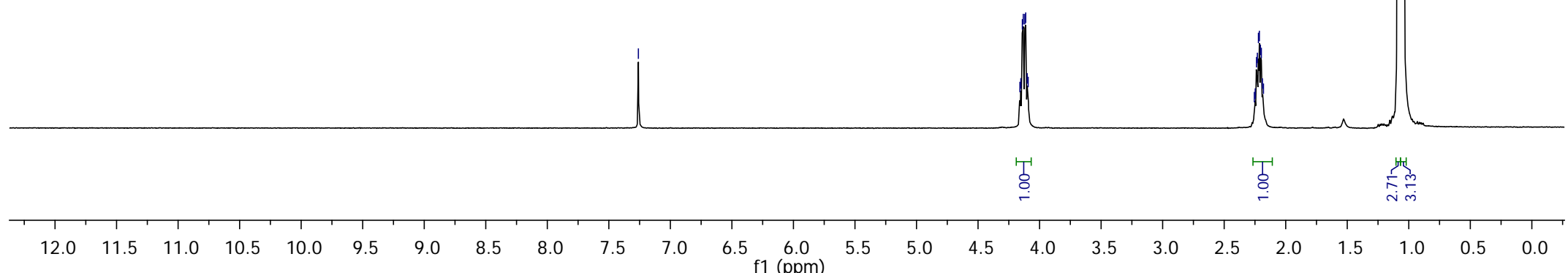




$$
{ }_{\mathrm{Br}} \mathrm{C}_{\mathrm{CF}_{3}}
$$

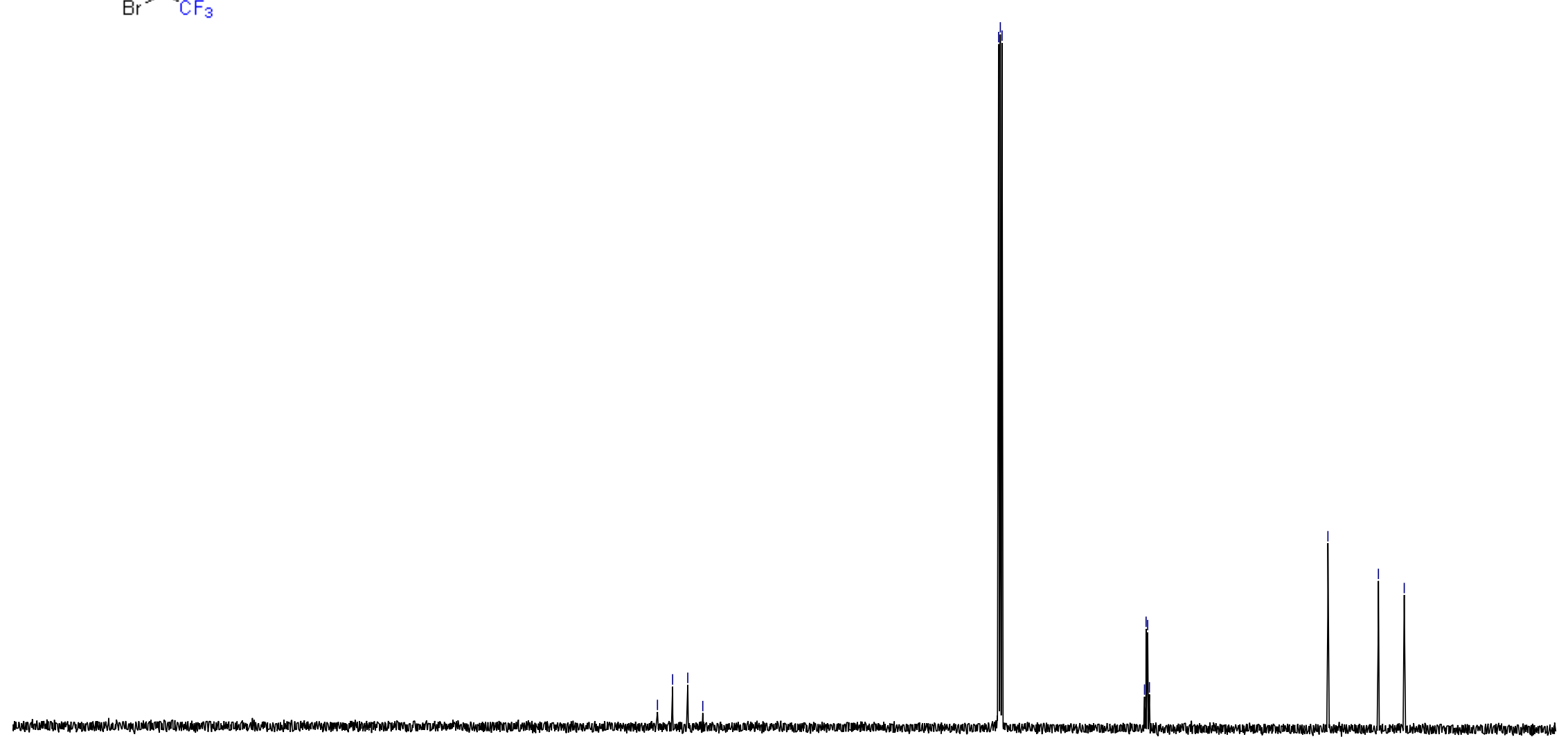



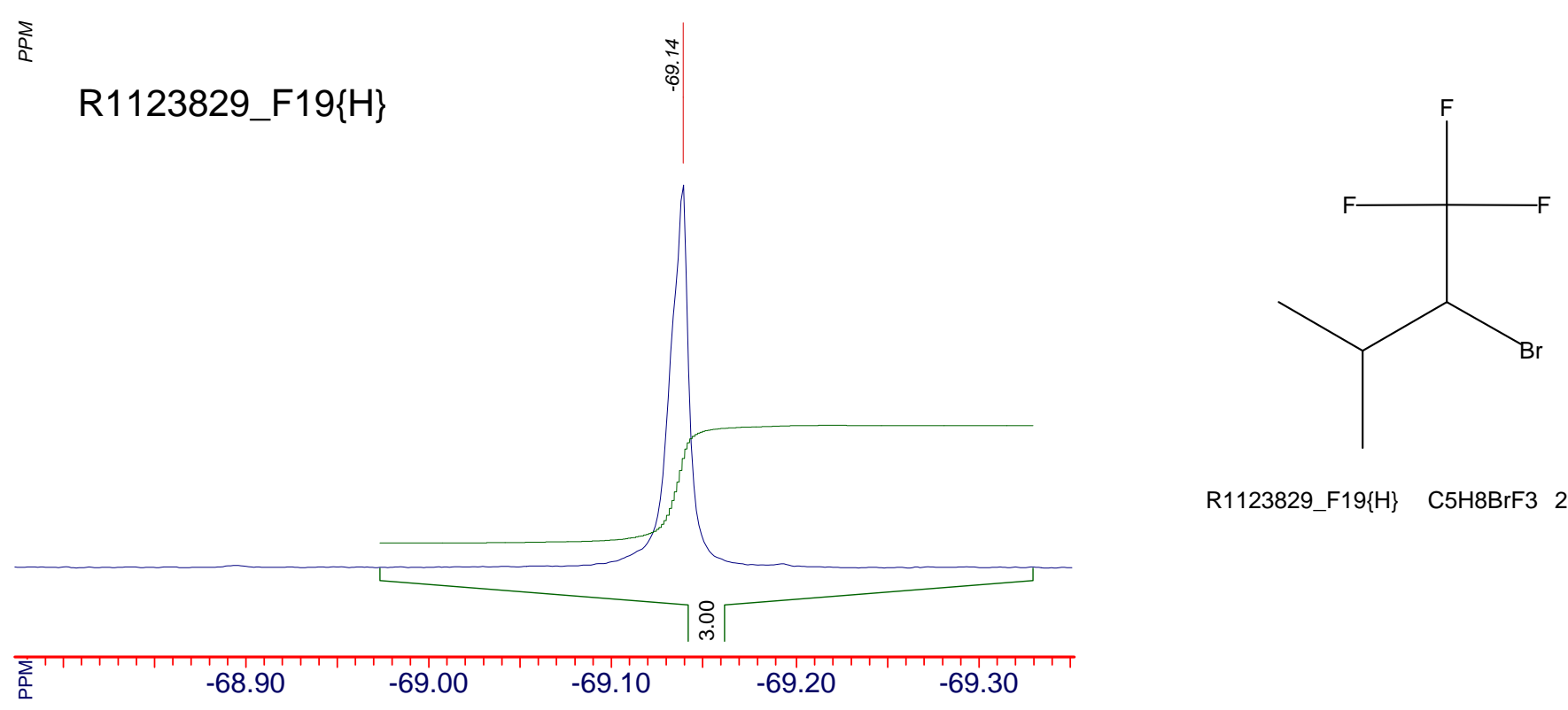

R1123829_F19\{H\} C5H8BrF3 205.02

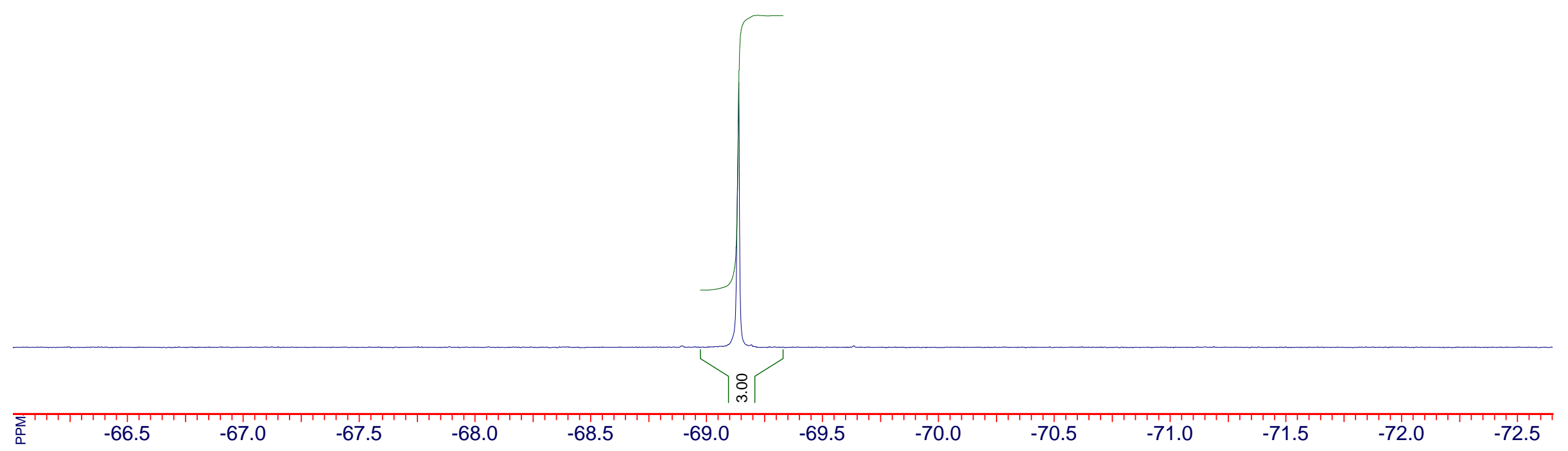




\section{Compound 20a}

R1207872

$\mathcal{B r}_{\mathrm{CF}_{3}}^{/ 2}$

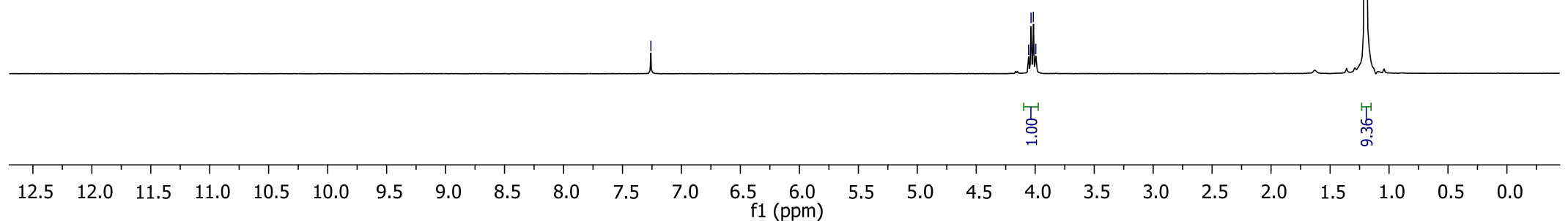


${ }_{\mathrm{Br}} \mathrm{C}_{\mathrm{CF}_{3}}$

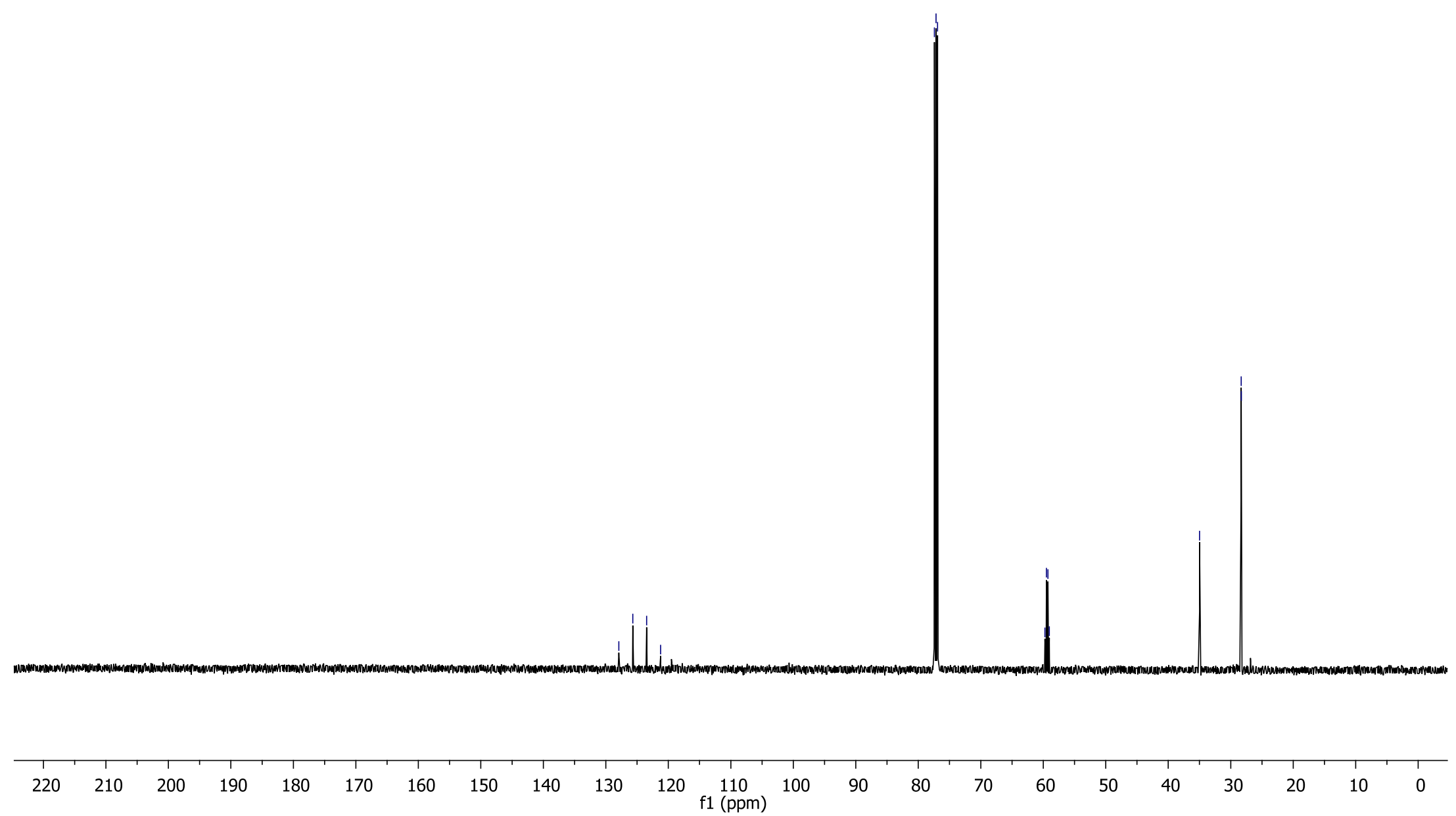


R1207872_F19 $\{H\}$

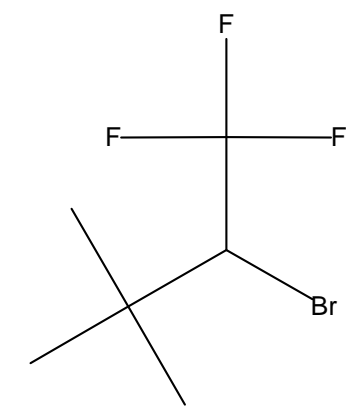

R1207872_F19\{H\} C6H10BrF3 219.04 


\section{Compound 21a}

igrk6993
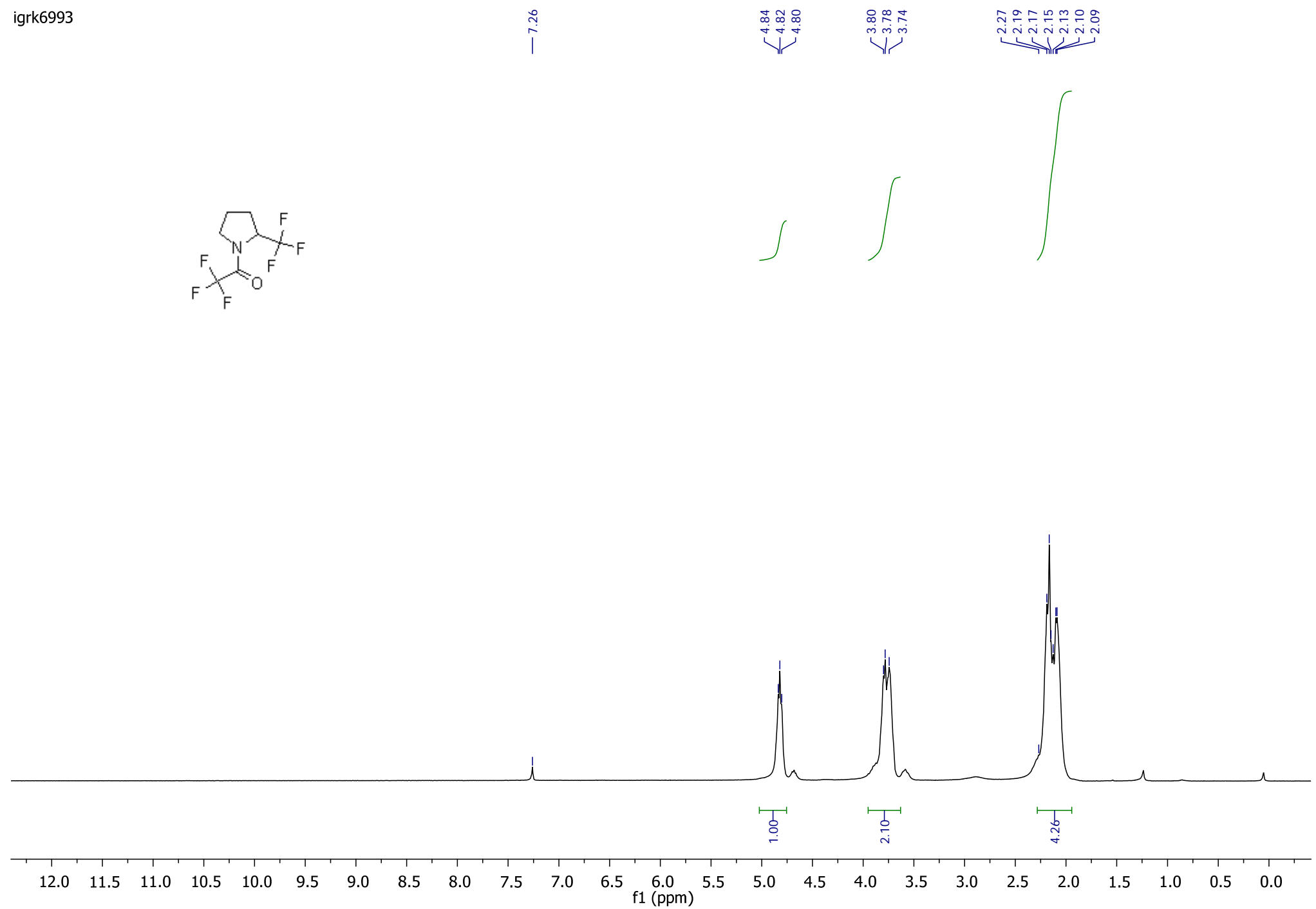
igrk6993_C13

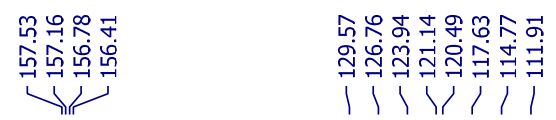

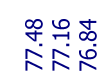

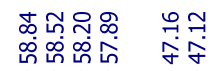

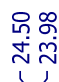

${ }_{F} \sum_{F}^{F}$

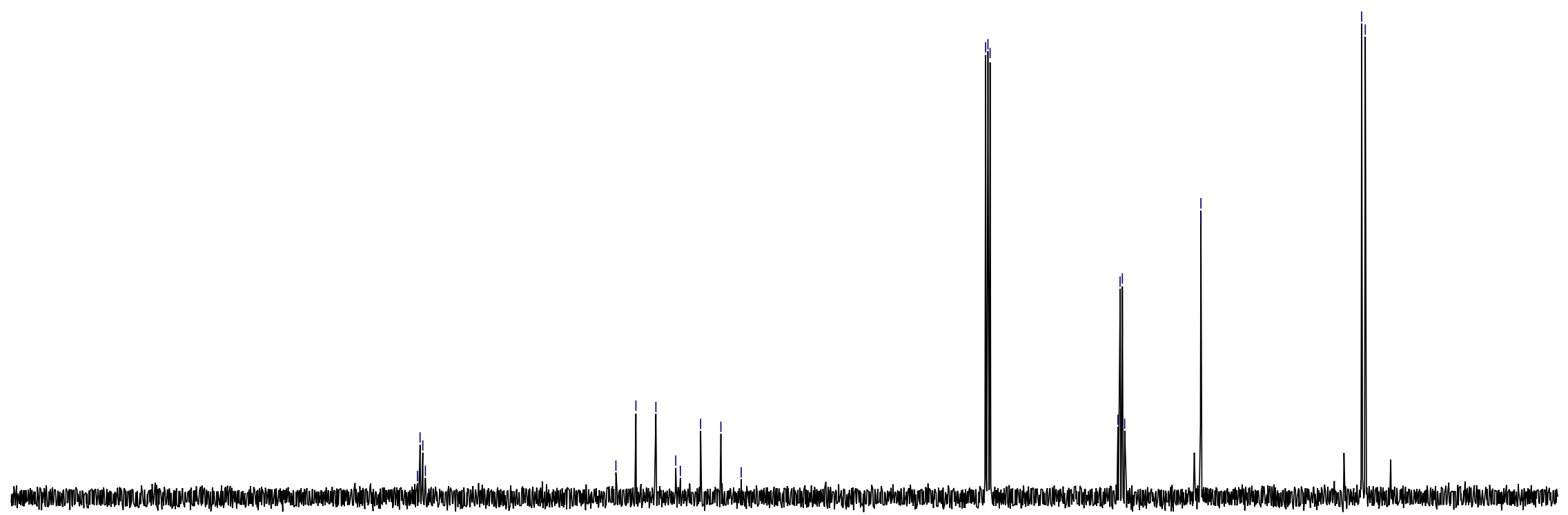

$210 \quad 200$

$190 \quad 180$

170

150

130

120

$110 \quad 100$

90

80

70

60

50

$40 \quad 30$

20 0 
igrk6993_F19 $\{\mathrm{H}\}$

$19 F\{1 \mathrm{H}\}$
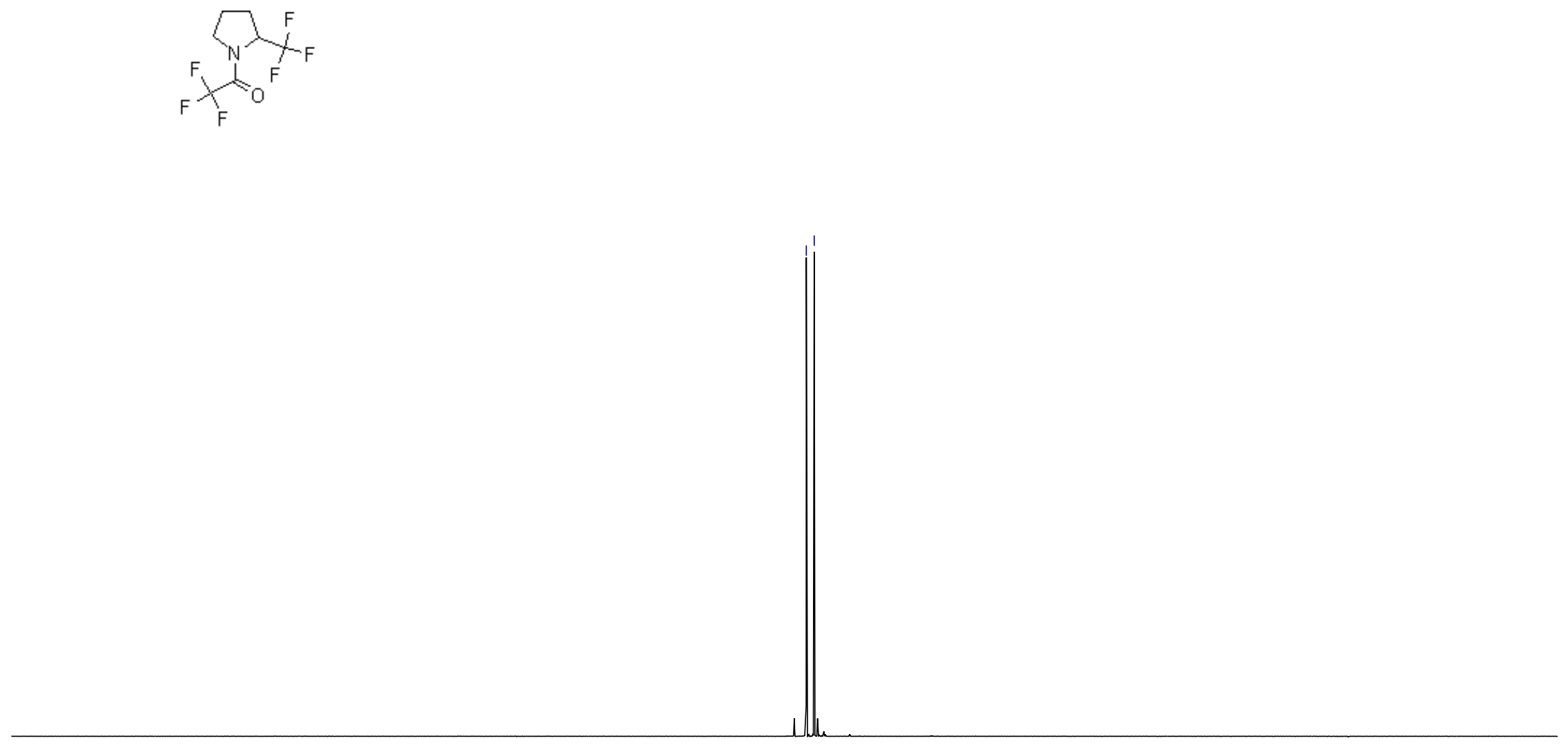

\begin{tabular}{llllllllllllllllllllllll}
\hline & 80 & 70 & 60 & 50 & 40 & 30 & 20 & 10 & 0 & -10 & -20 & -30 & -40 & -50 & -60 & -70 & -80 & -90 & -100
\end{tabular} 


\section{Compound 22a}

R674283

$\mathrm{O} \underset{\mathrm{H}}{\mathrm{CF}_{3}}$

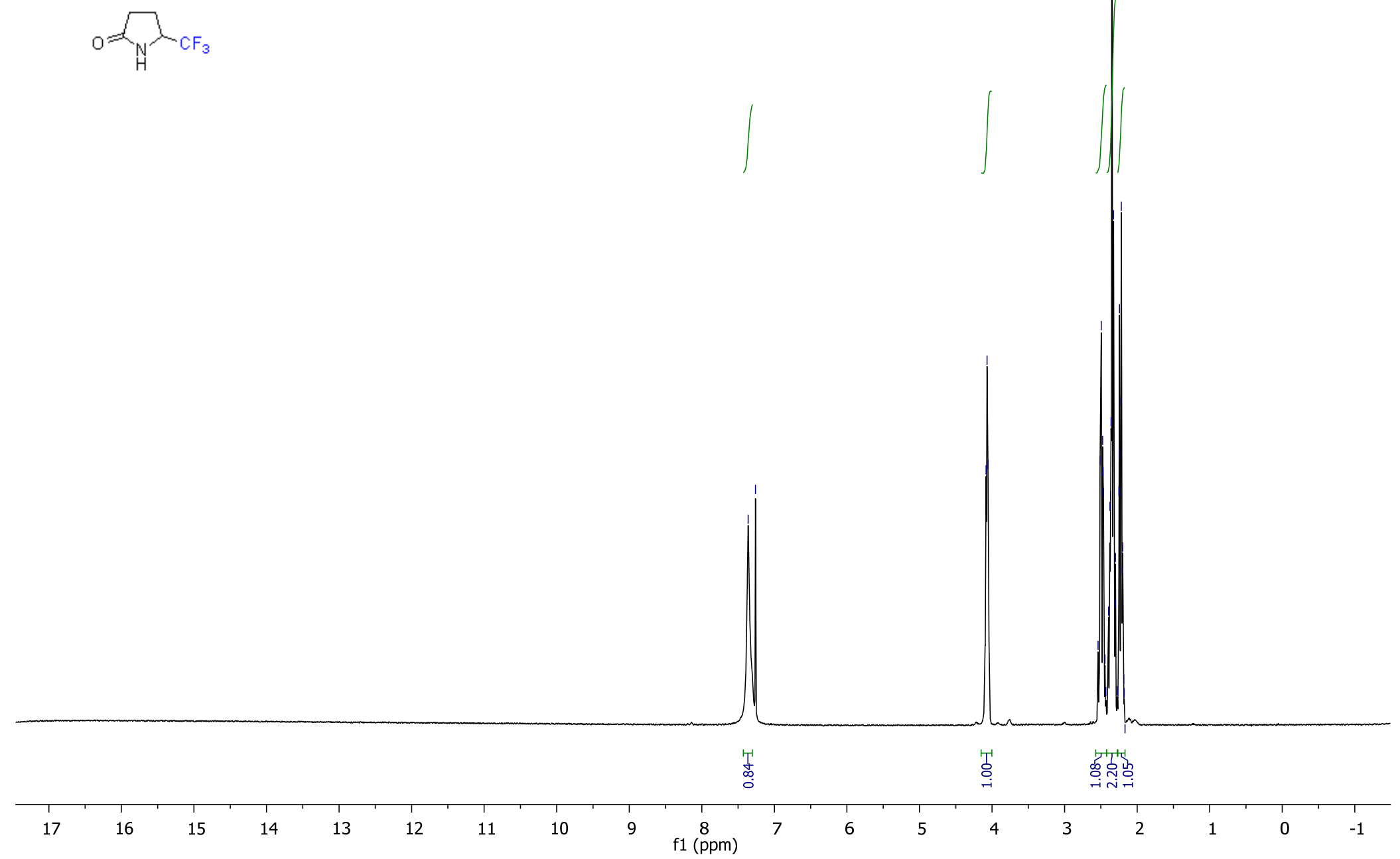


R674283_C13

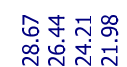

१iा
굿영

战

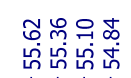

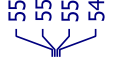

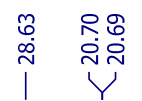<smiles>O=C1CCC(C(F)(F)F)N1</smiles>

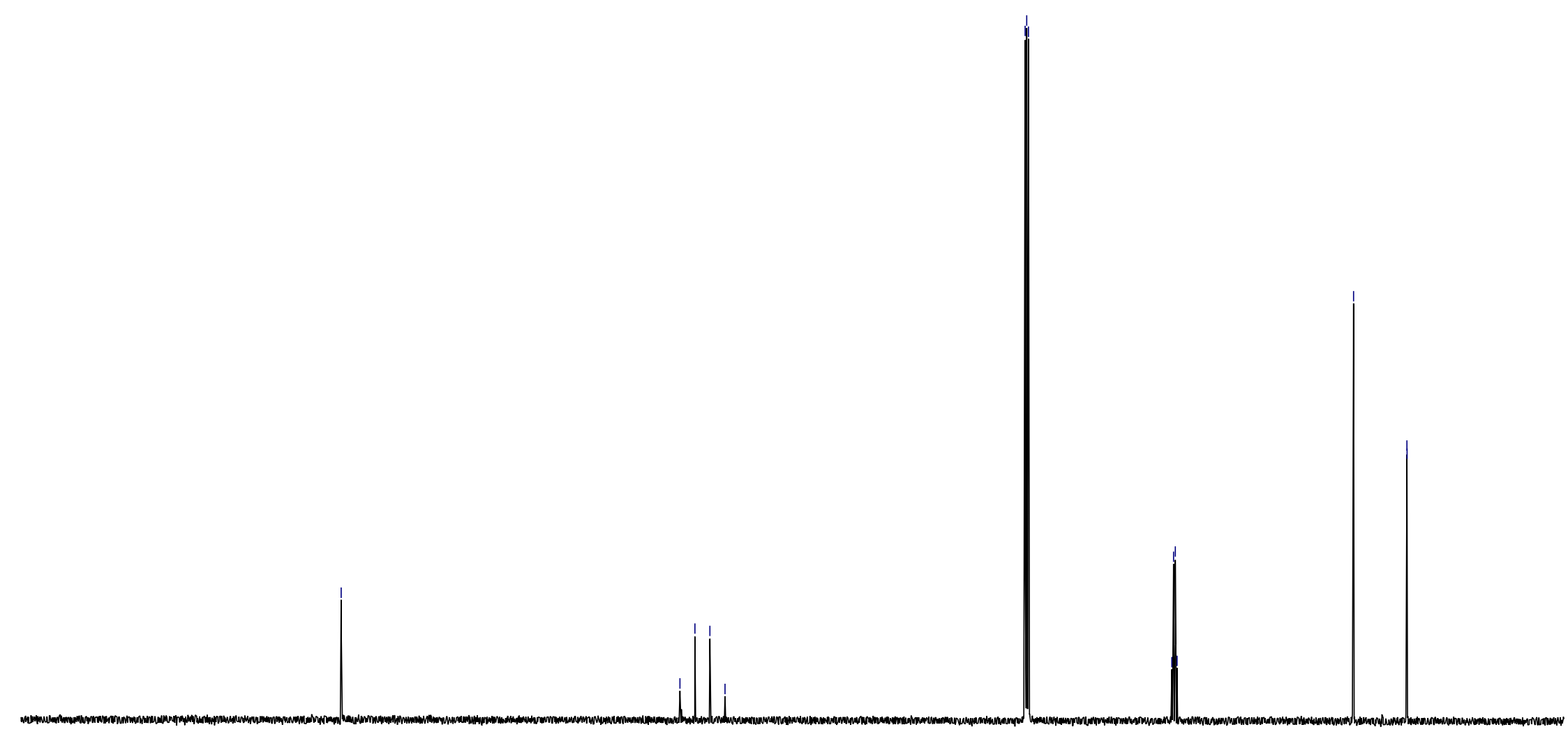


$O={ }_{\mathrm{H}} \mathrm{CF}_{3}$

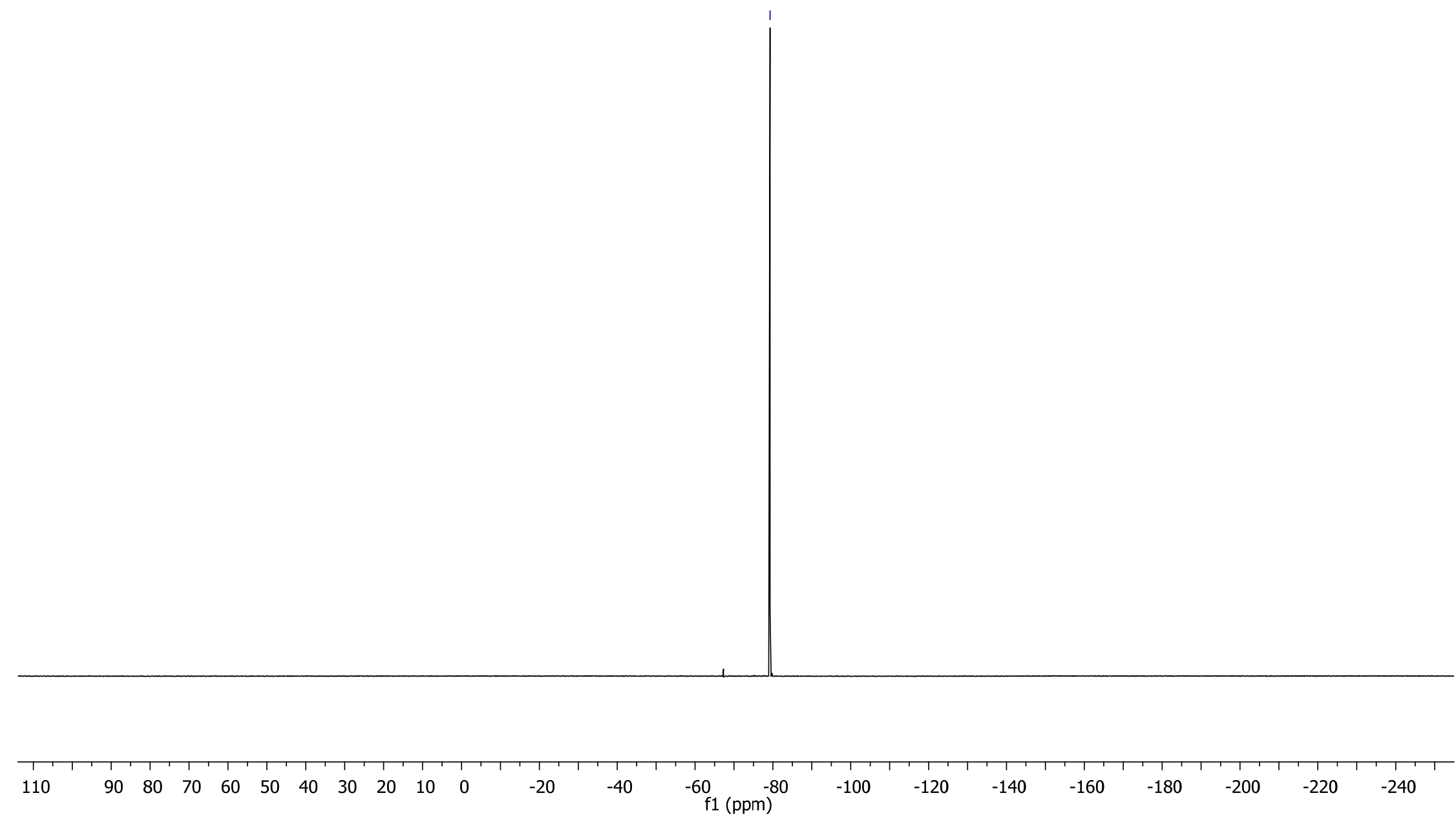




\section{Compound 23a}

R1069478

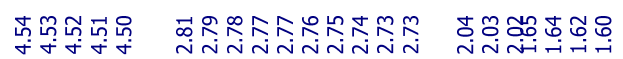

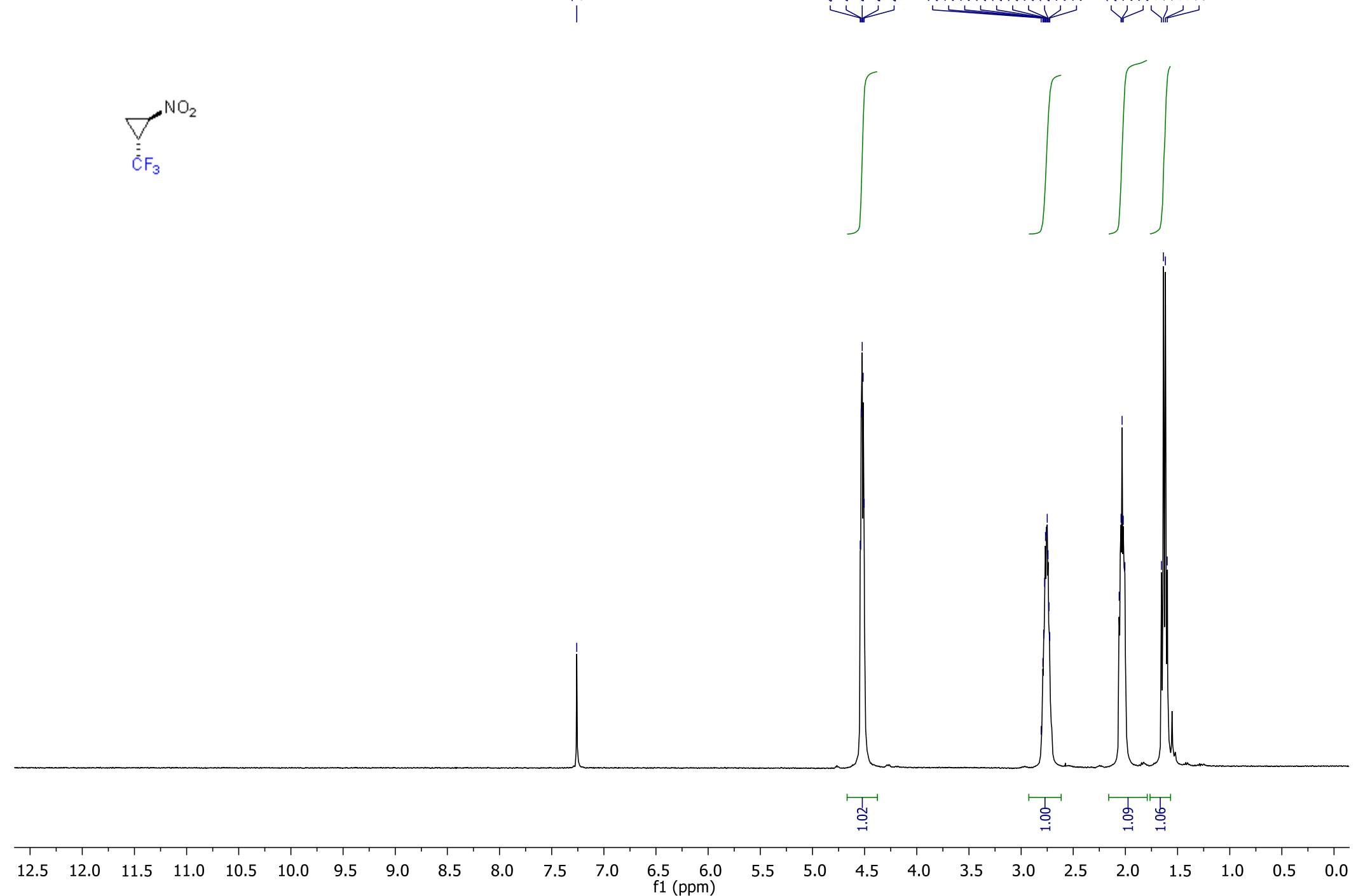




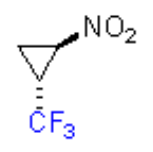

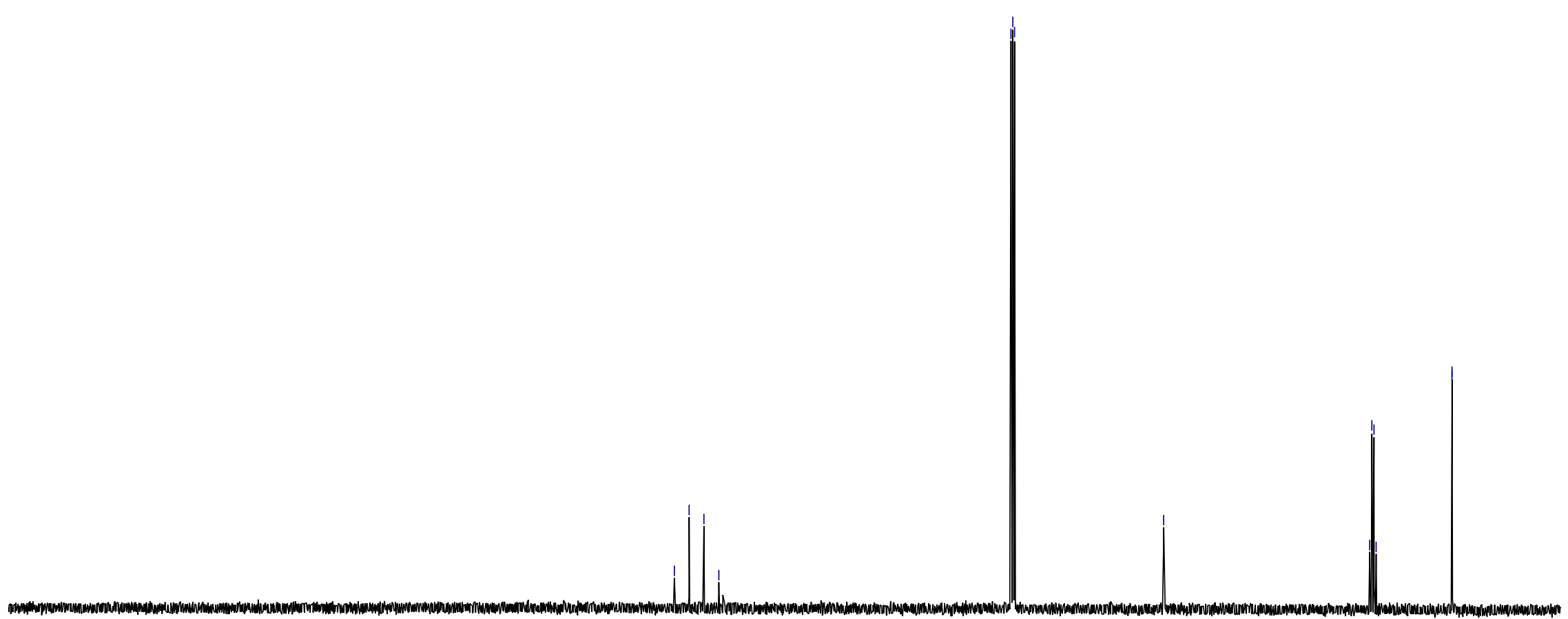


R1069478_F19 $\{\mathrm{H}\}$
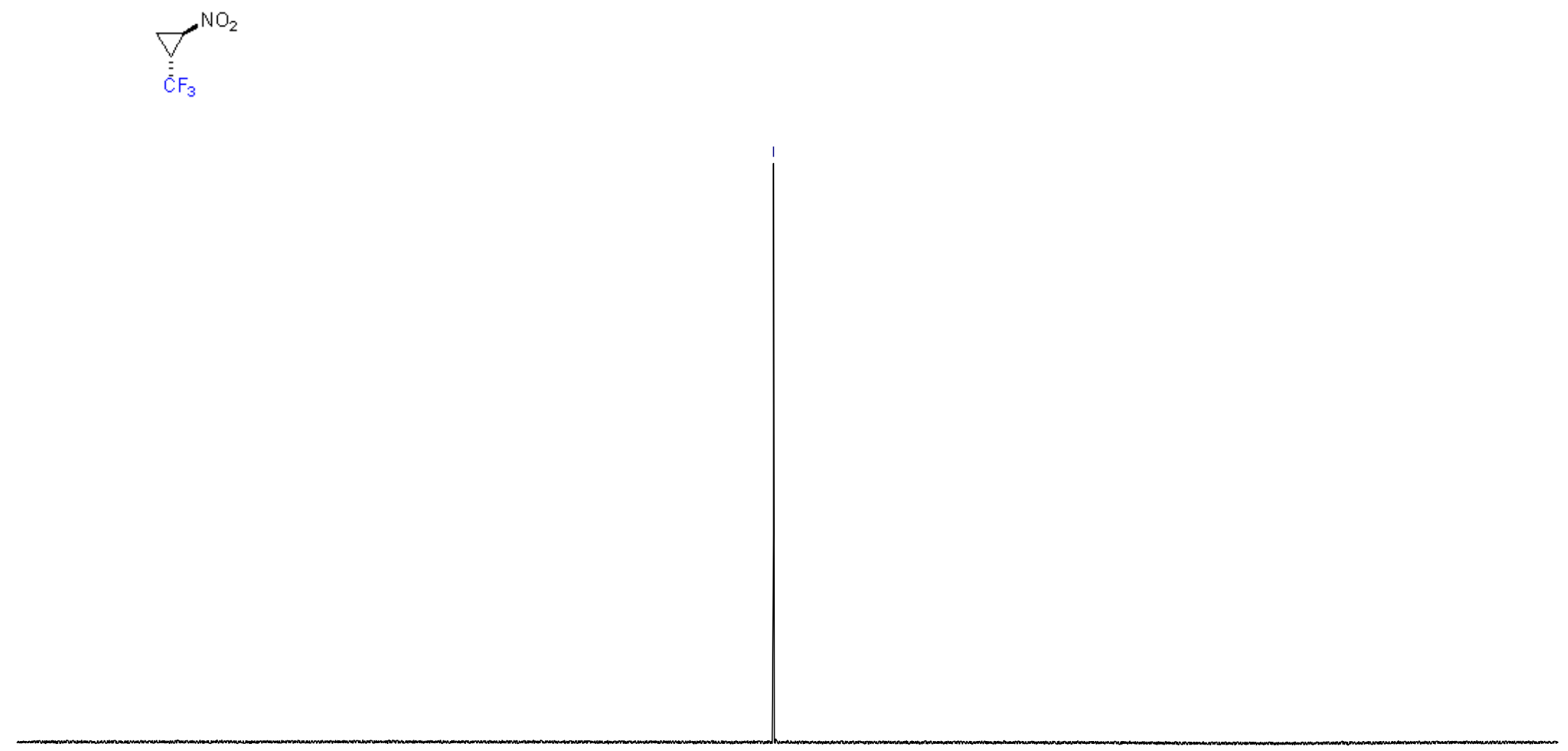


\section{Compound 24a}

R590303

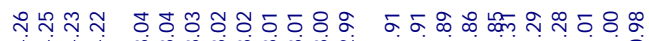

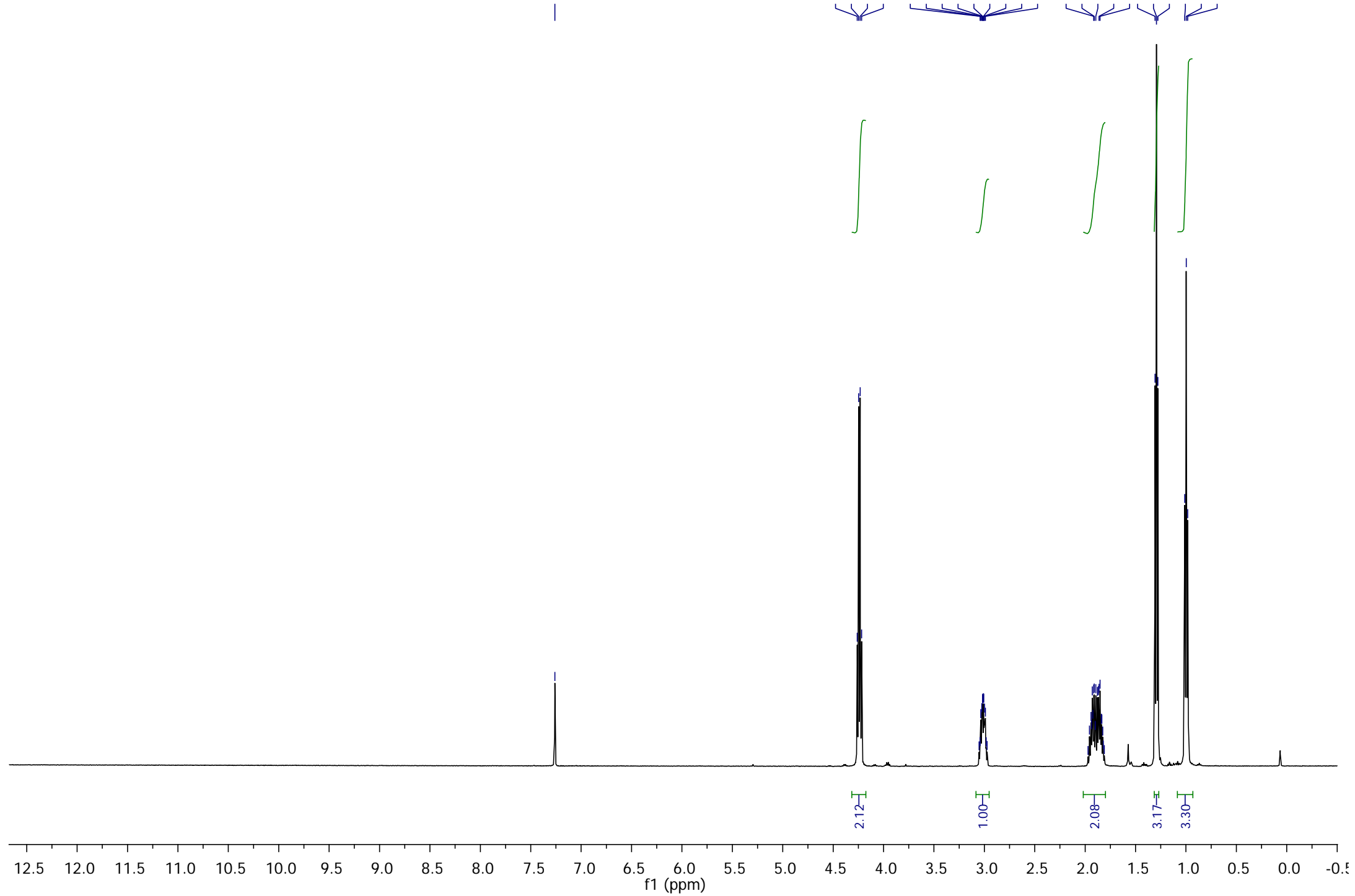




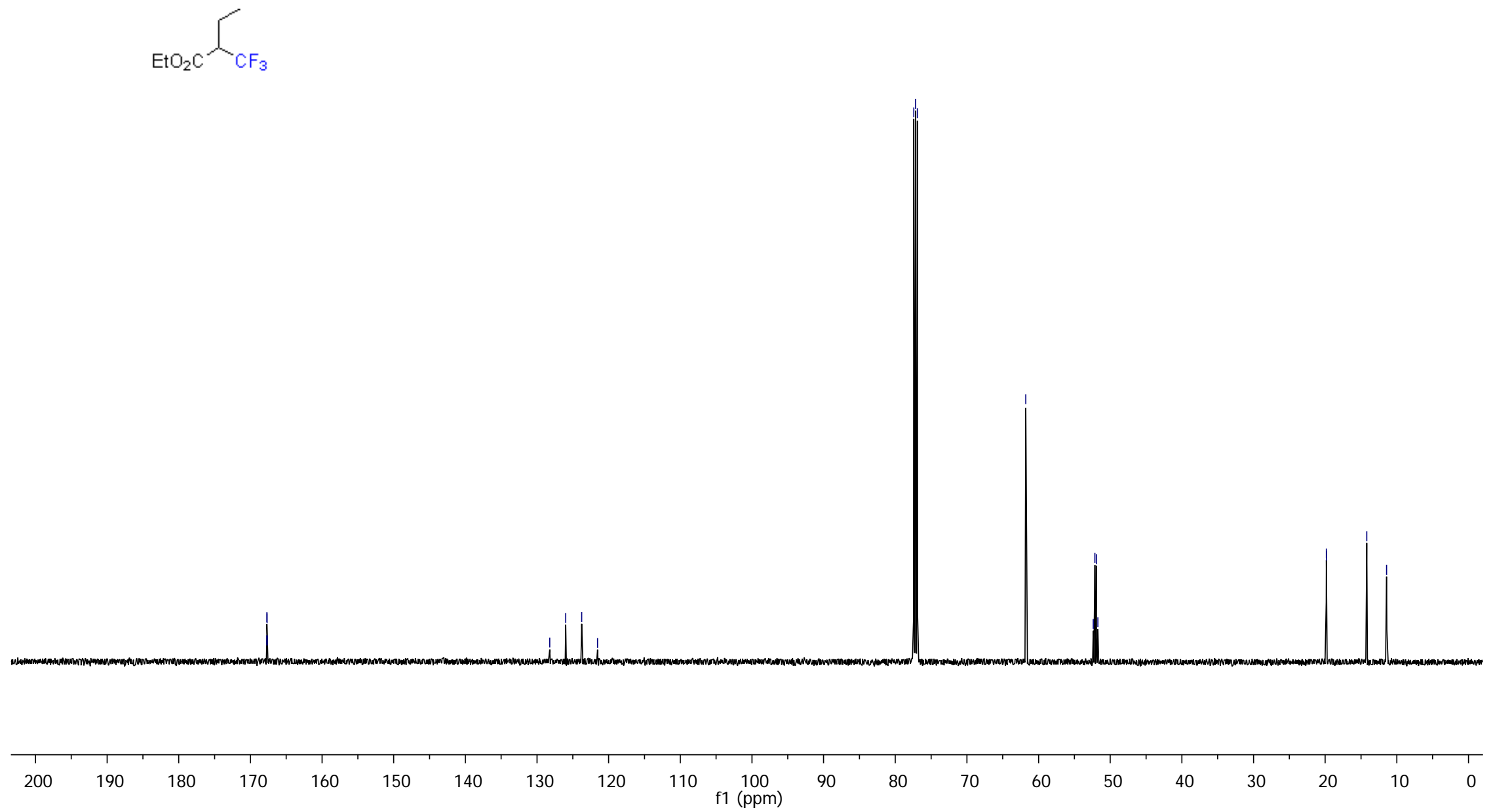


R590303_F19

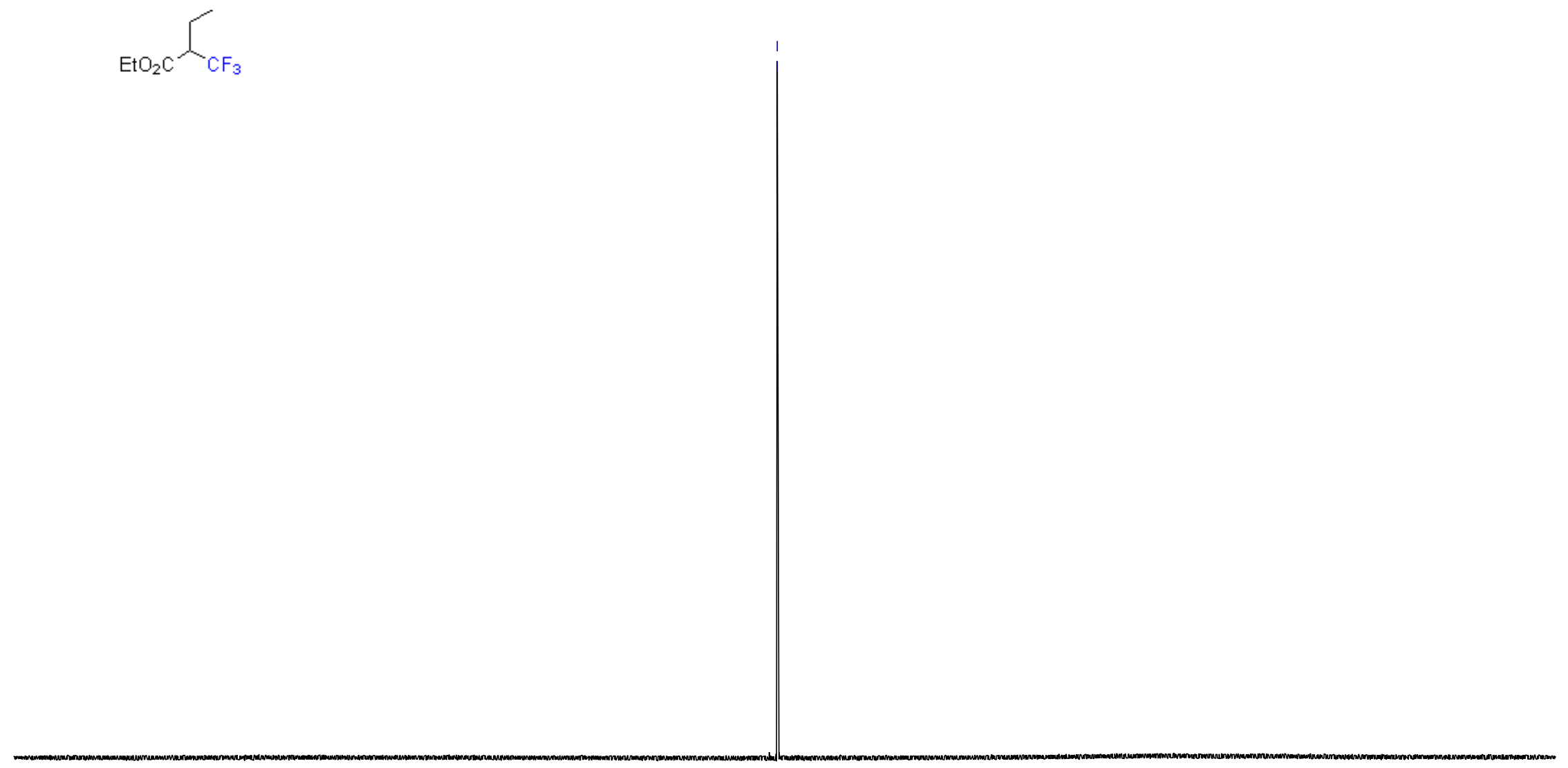

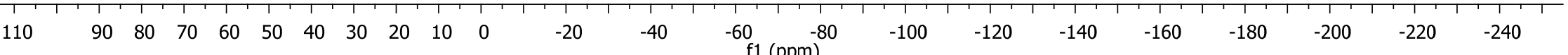




\section{Compound 25a}

R656779

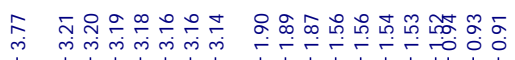<smiles>[M]C(C)CC(C(C)=O)C(F)(F)F</smiles>

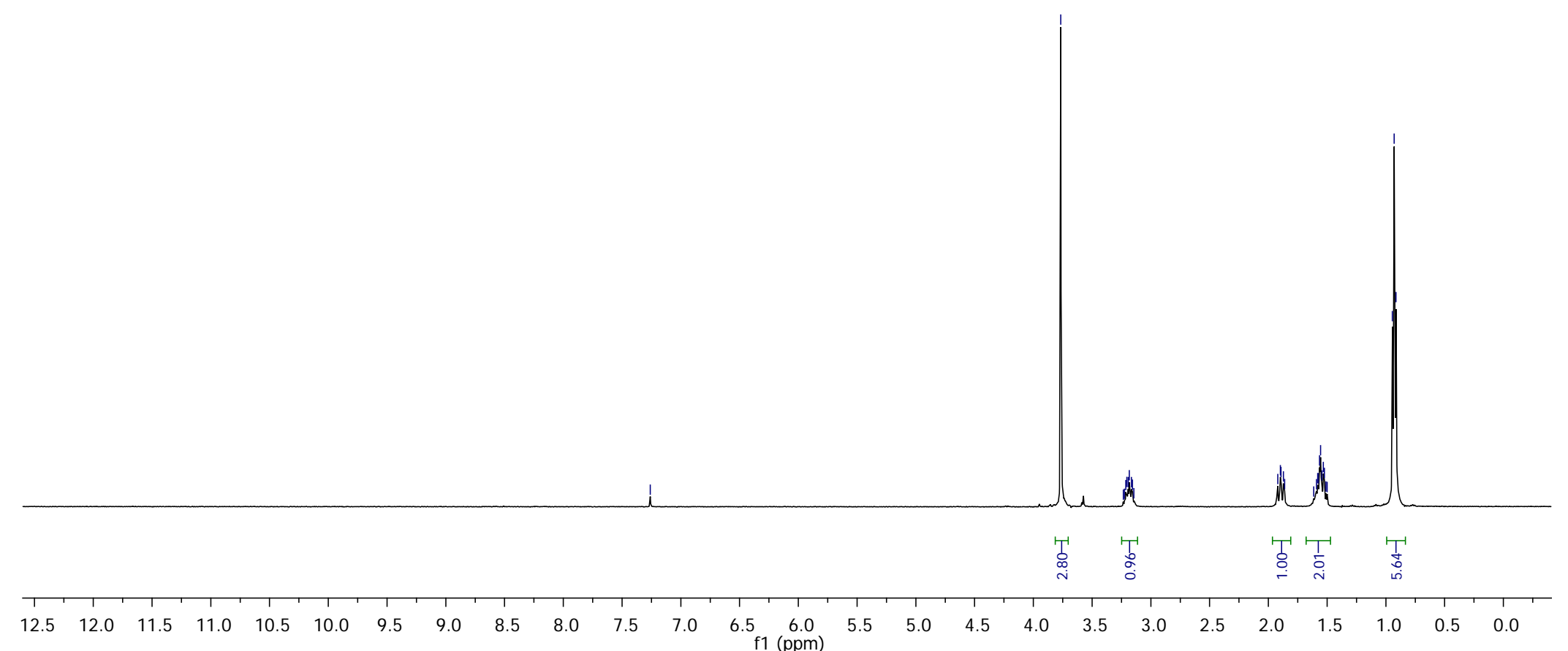


R656779_C1

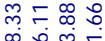

웛워

१1।

$\int_{\mathrm{MeO}_{2} \mathrm{C}}^{\mathrm{Me}} \mathrm{C}_{\mathrm{Me}}$

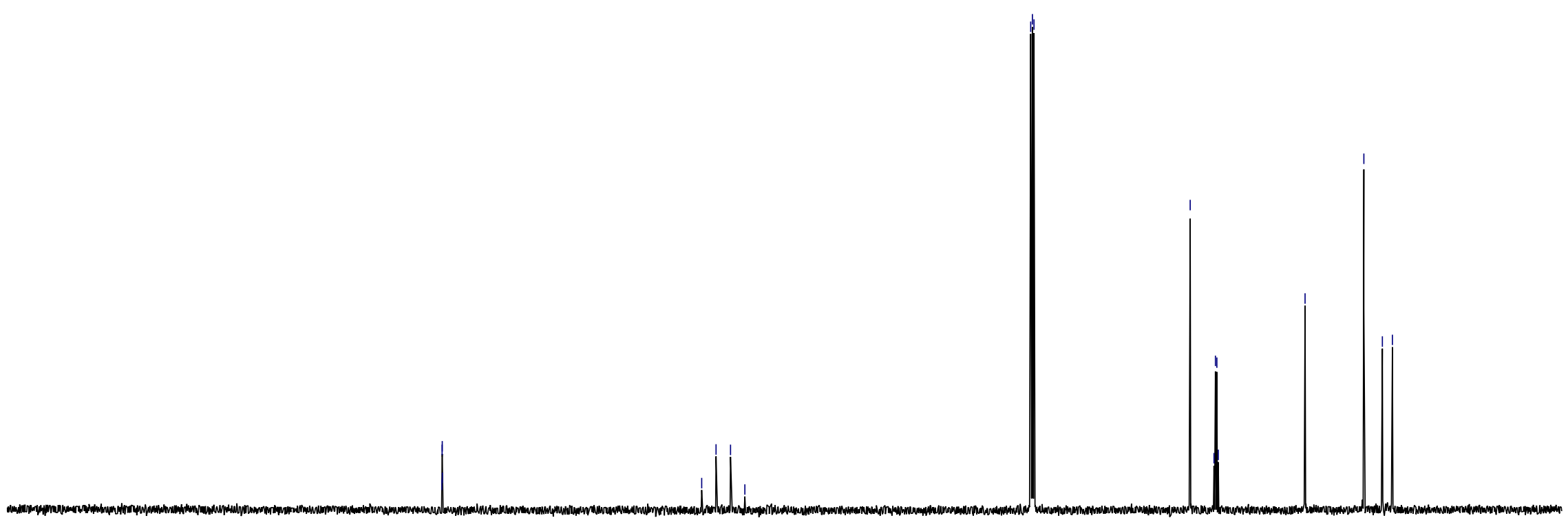

$\begin{array}{lllllllllllll}230 & 220 & 210 & 200 & 190 & 180 & 170 & 160 & 150 & 140 & 130 & 120 & 110\end{array}$ f1 (ppm)

\section{平哭}

$\sqrt{i=0}$

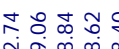

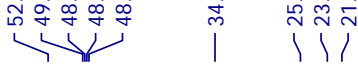

䑻 
R656779_F19

F19

${ }_{\mathrm{MeO}_{2} \mathrm{C}}^{\mathrm{Me}} \overbrace{\mathrm{CF}_{3}}^{\mathrm{Me}}$

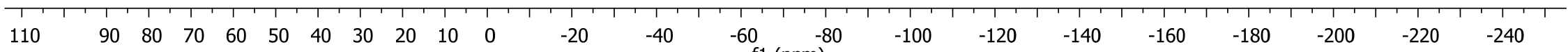




\section{Compound 26a}

R308073

$$
\mathrm{Br} \stackrel{\mathrm{Ce}}{\mathrm{Me}}_{\mathrm{CF}_{3}}
$$
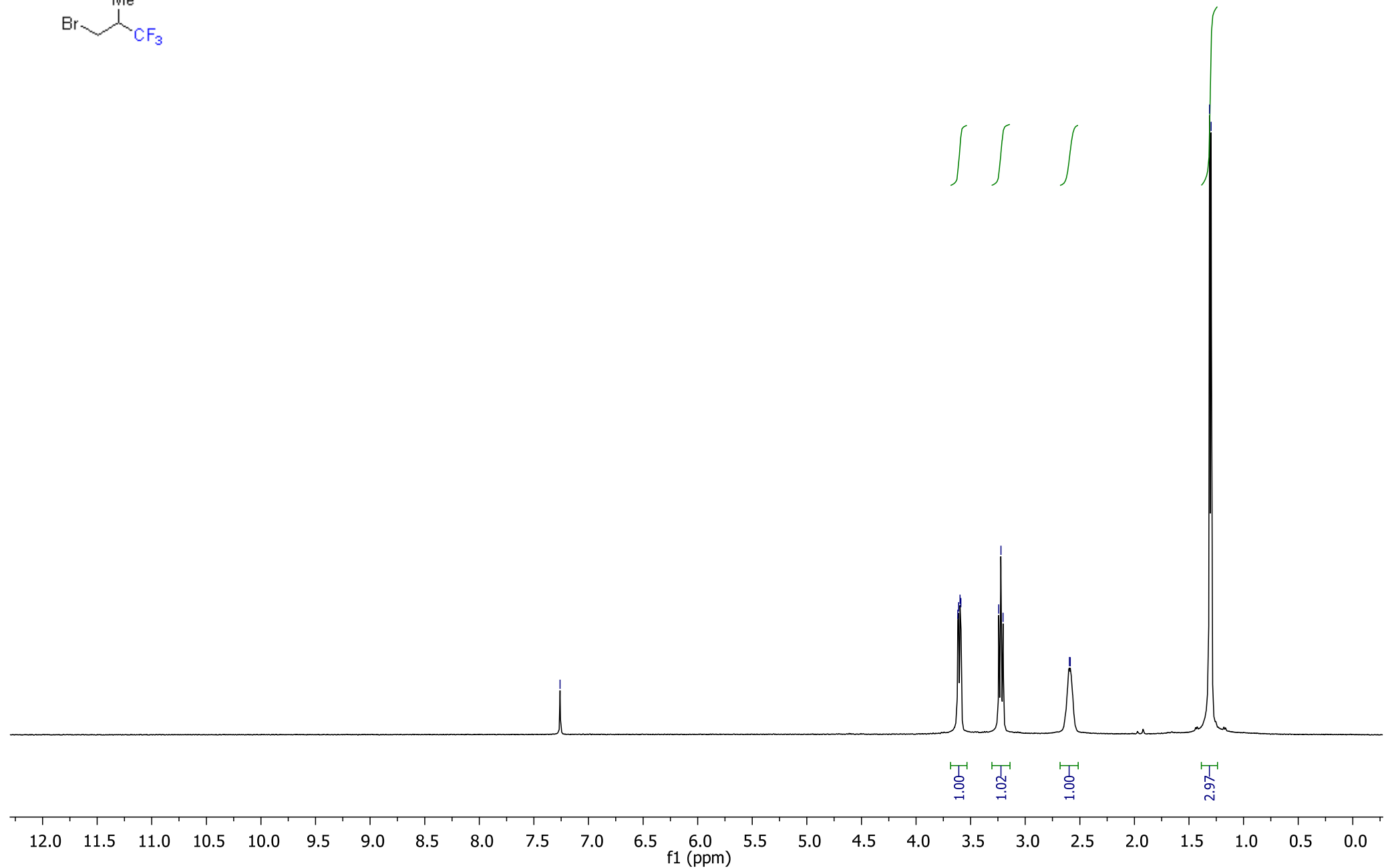
$\mathrm{Cr}_{\mathrm{CF}_{3}}^{\mathrm{Me}}$

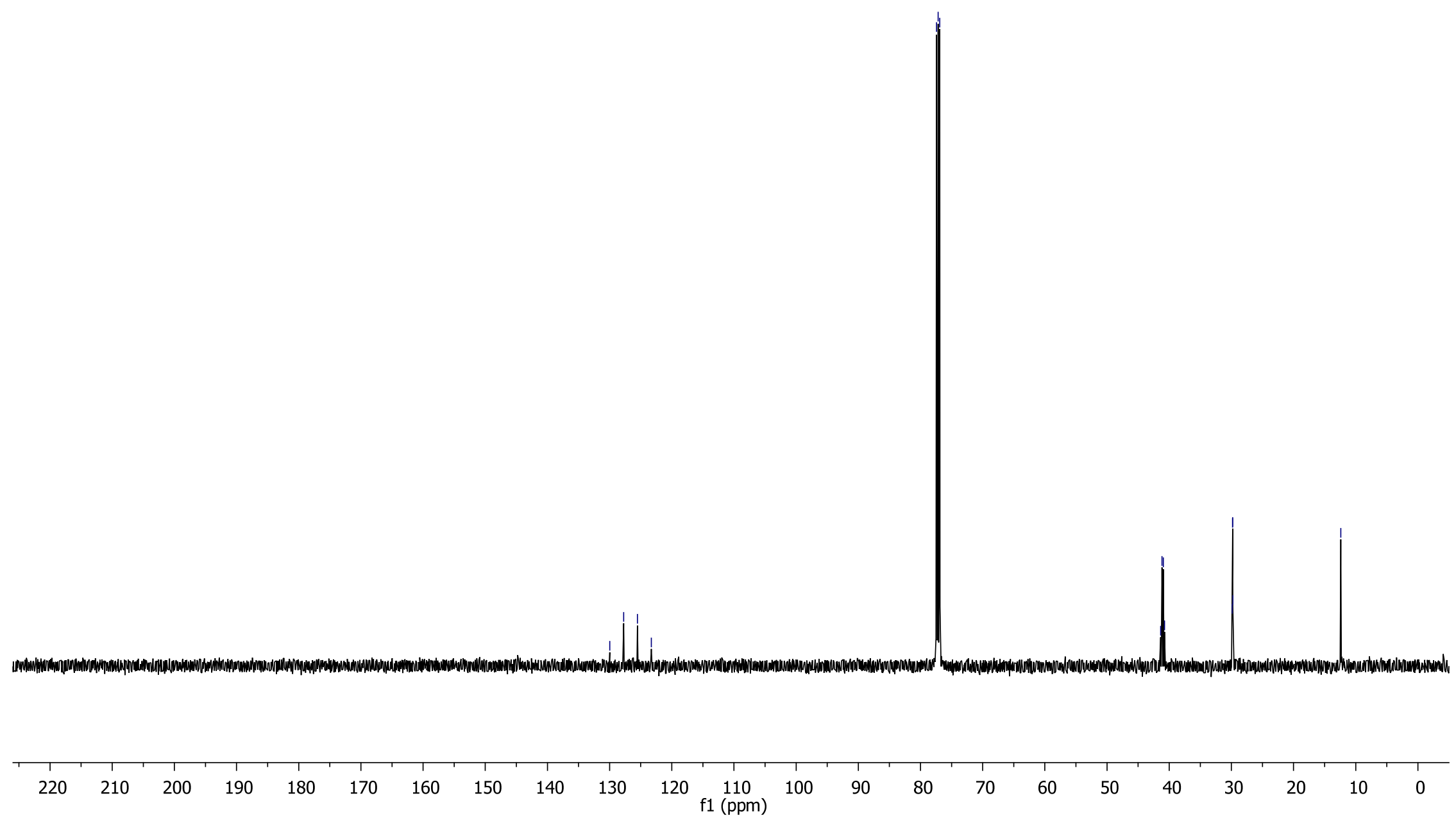




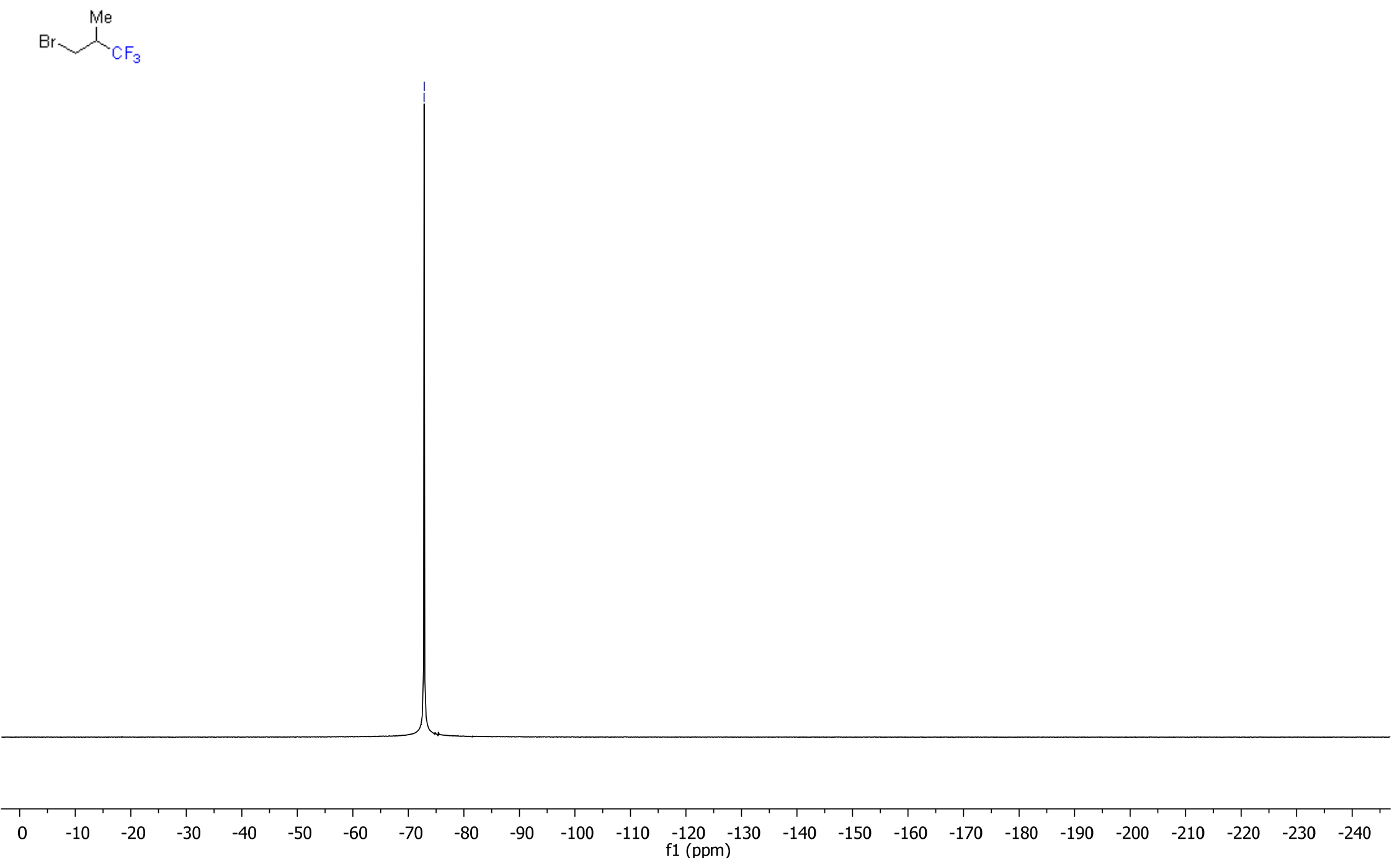




\section{Compound 27a}

R1366506

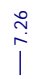

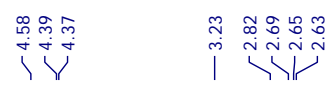

$\mathrm{Br} \longrightarrow \mathrm{CF}_{3}$
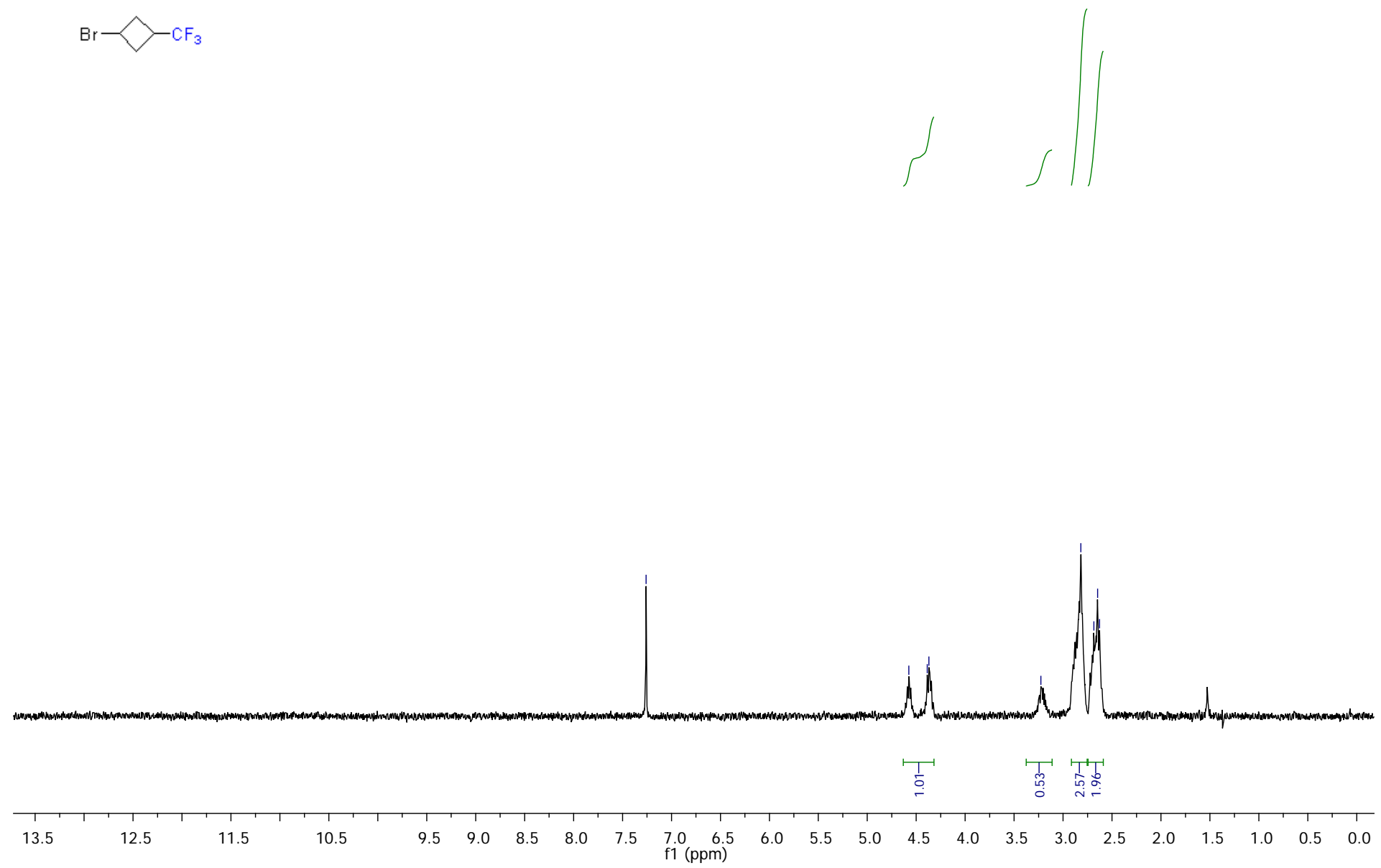

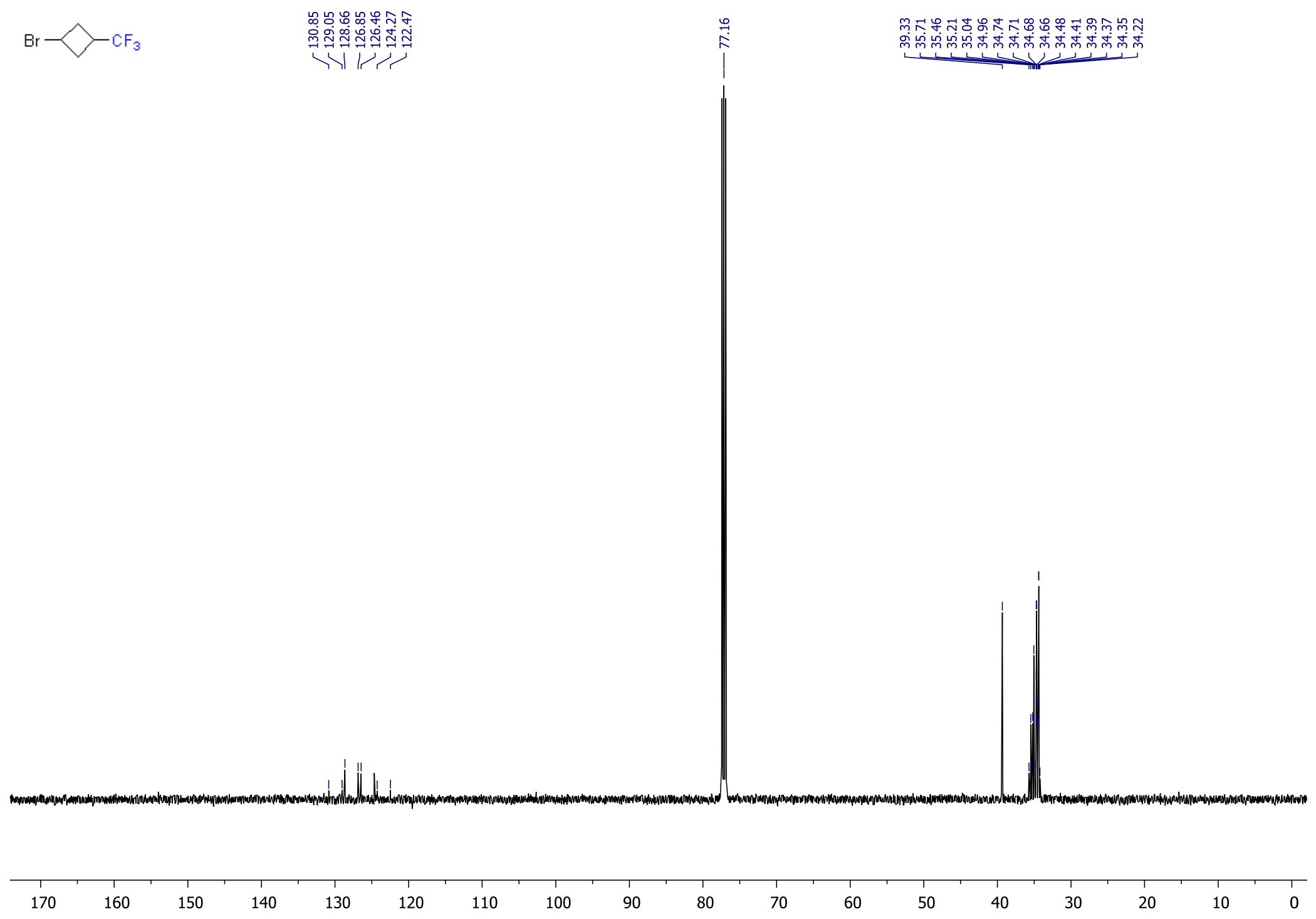
R1366506_F19 $\{\mathrm{H}\}$

$19 \mathrm{~F}-\{1 \mathrm{H}\}$

$\mathrm{Br} \prec \mathrm{CF}_{3}$

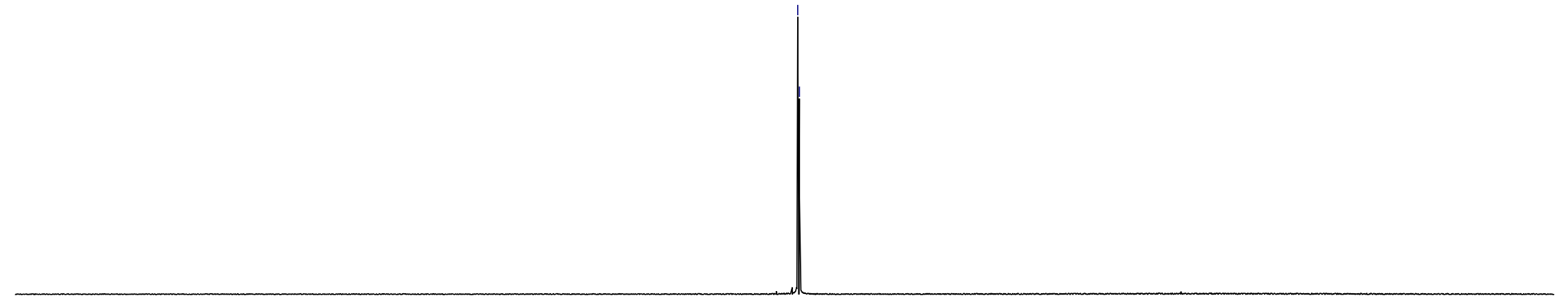

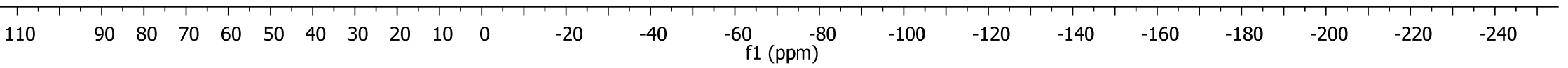




\section{Compound 28a}

R1199576

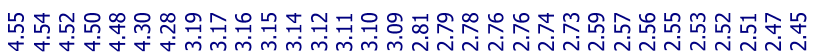

$\mathrm{Cl} \prec \mathrm{CF}_{3}$
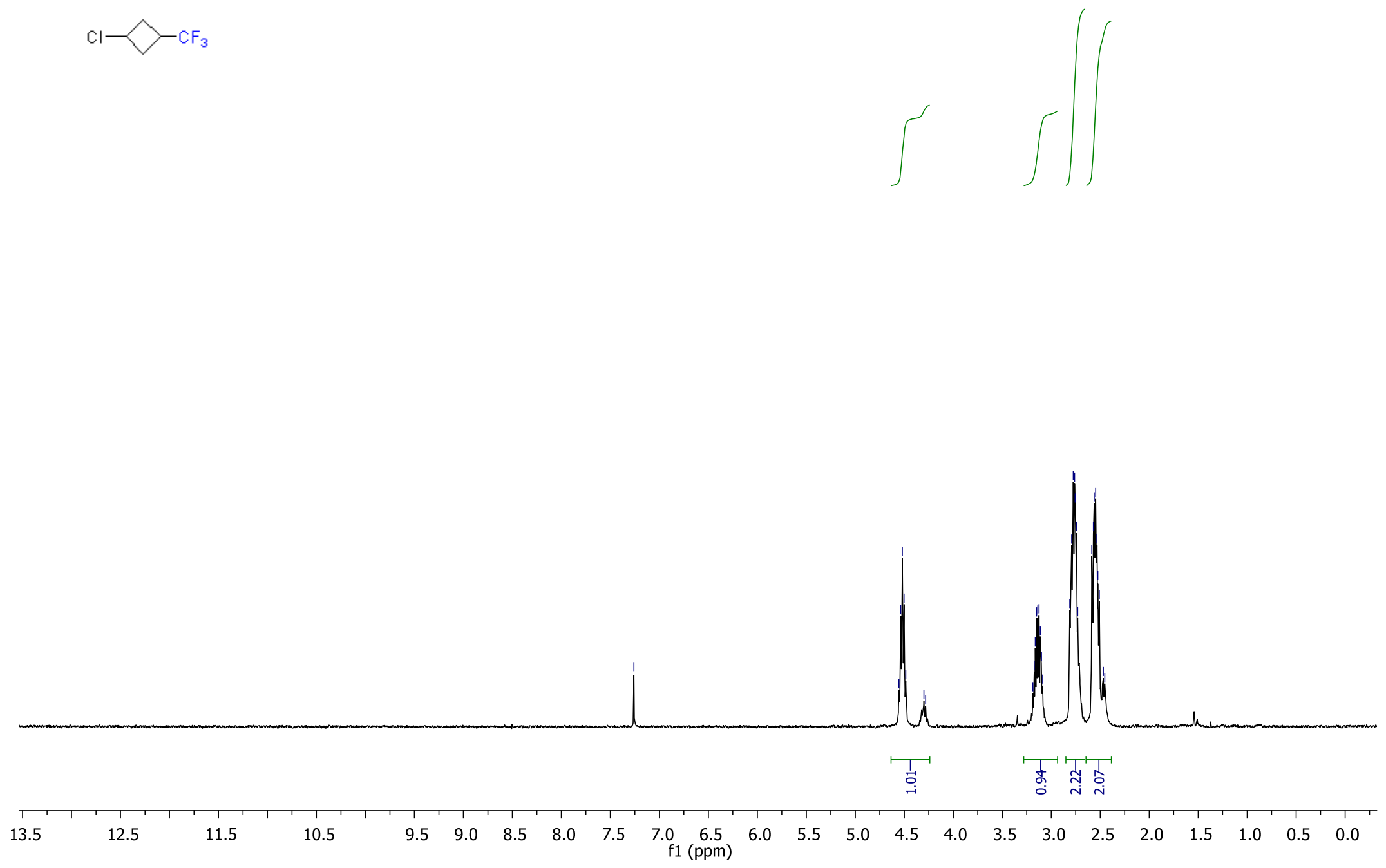
R1199576_C13

$\mathrm{Cl} \prec-\mathrm{CF}_{3}$
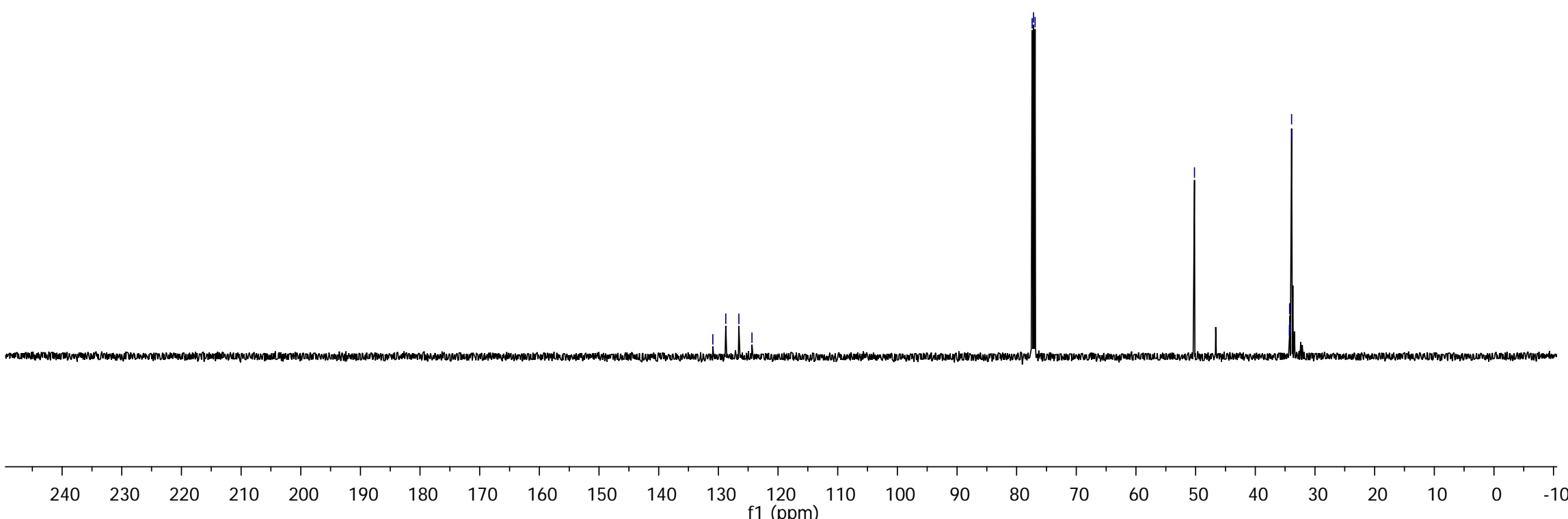
R1199576_F19 $\mathrm{H}\}$

$19 \mathrm{~F}\{1 \mathrm{H}\}$

$\mathrm{Cl} \longrightarrow \mathrm{CF}_{3}$

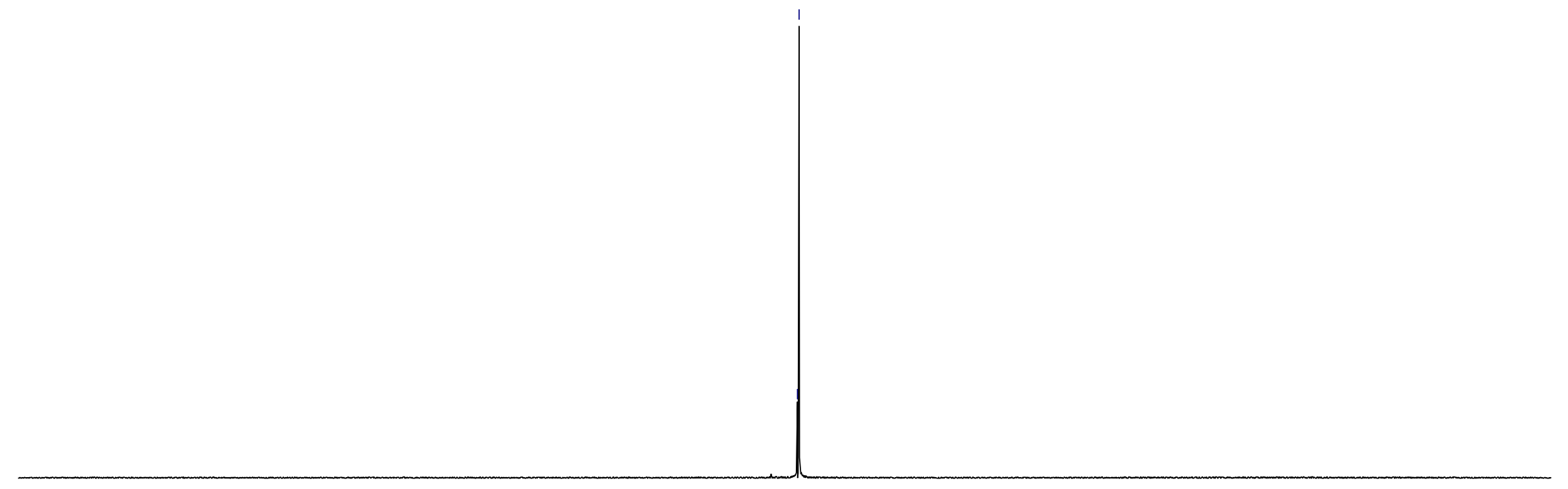




\section{Compound 29a}

mom-CF3az

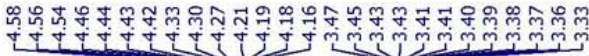

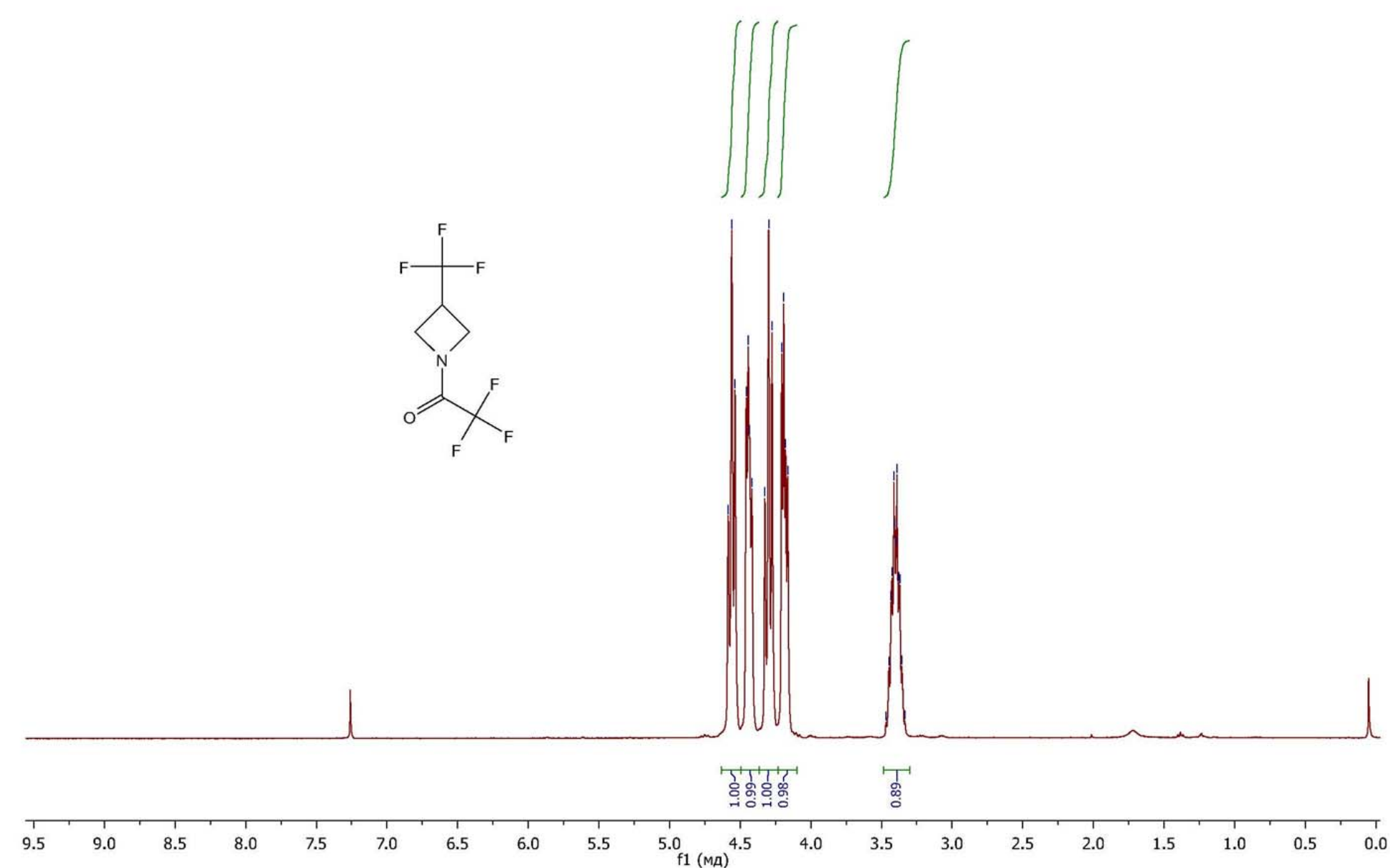




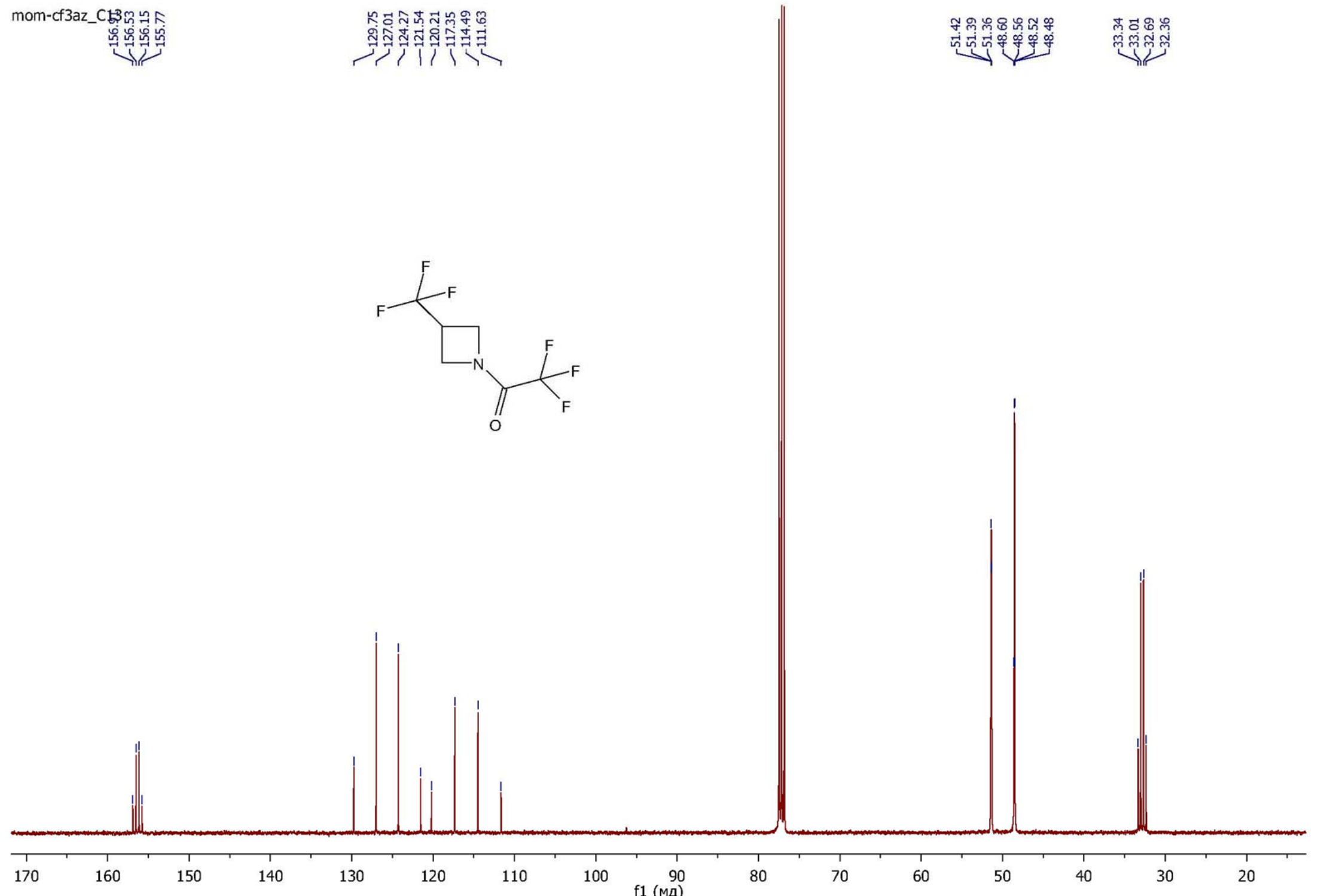


mom-CF3az-F19

$19 \mathrm{~F}$

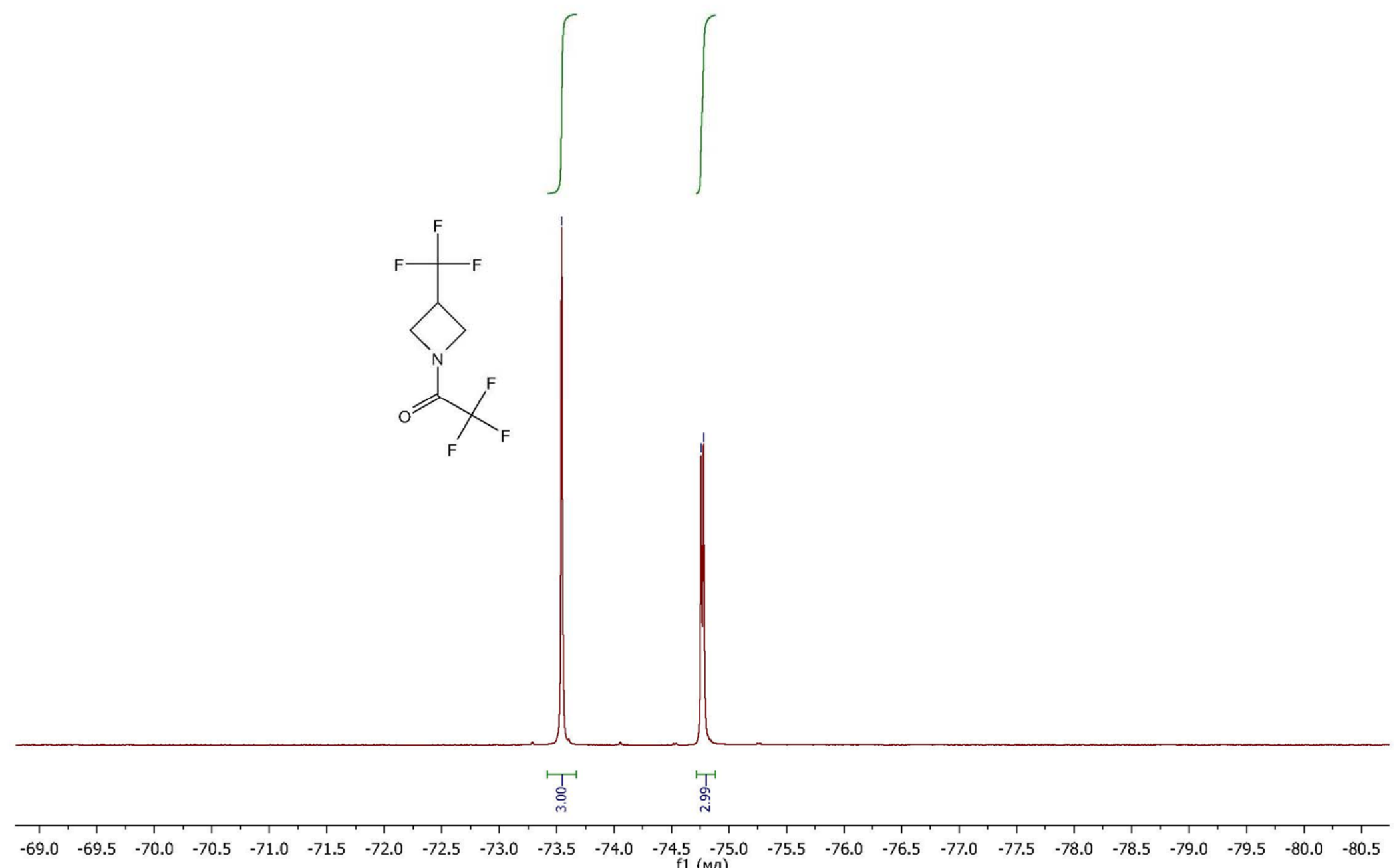




\section{Compound 30a}

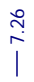

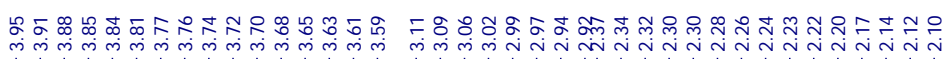
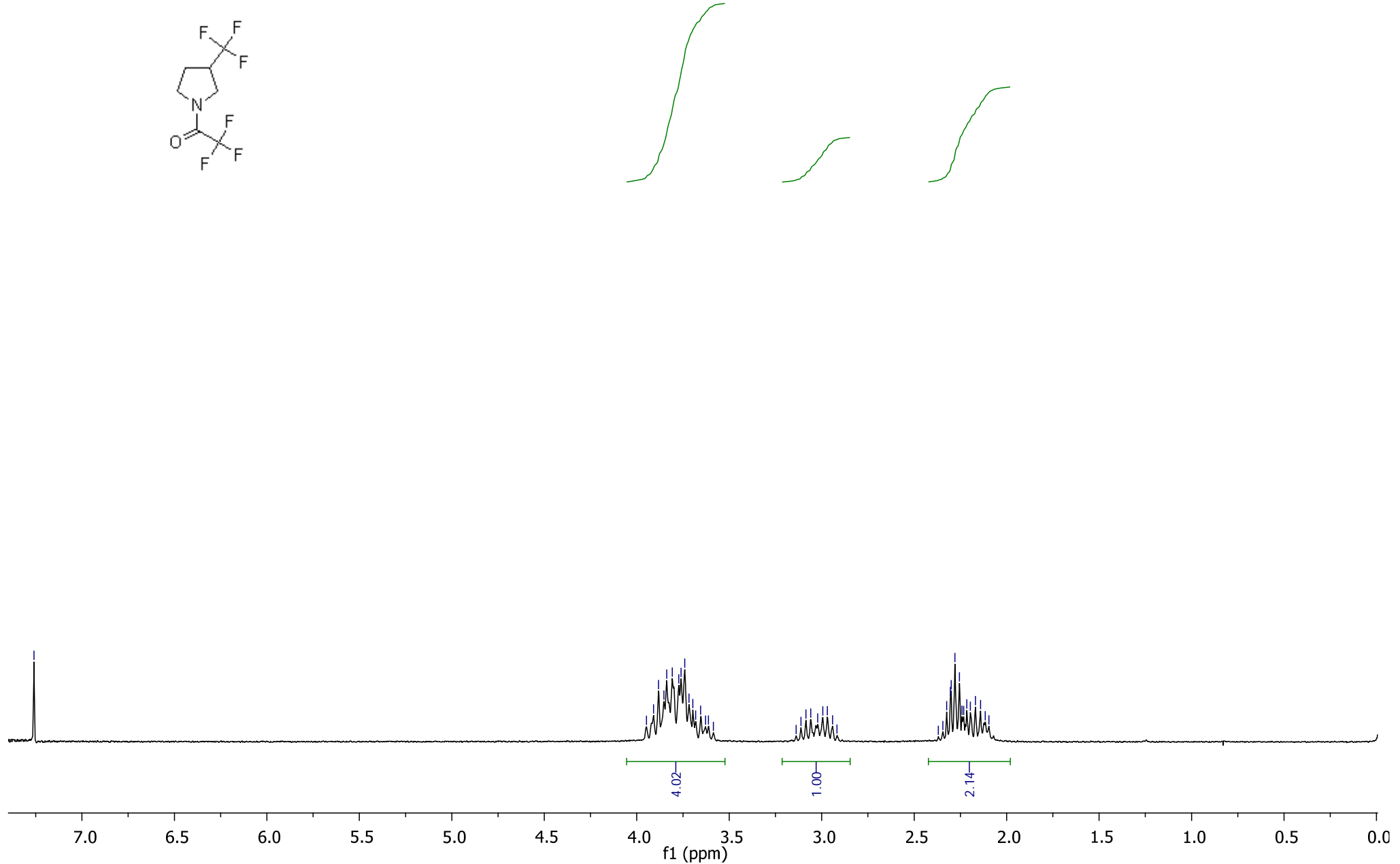


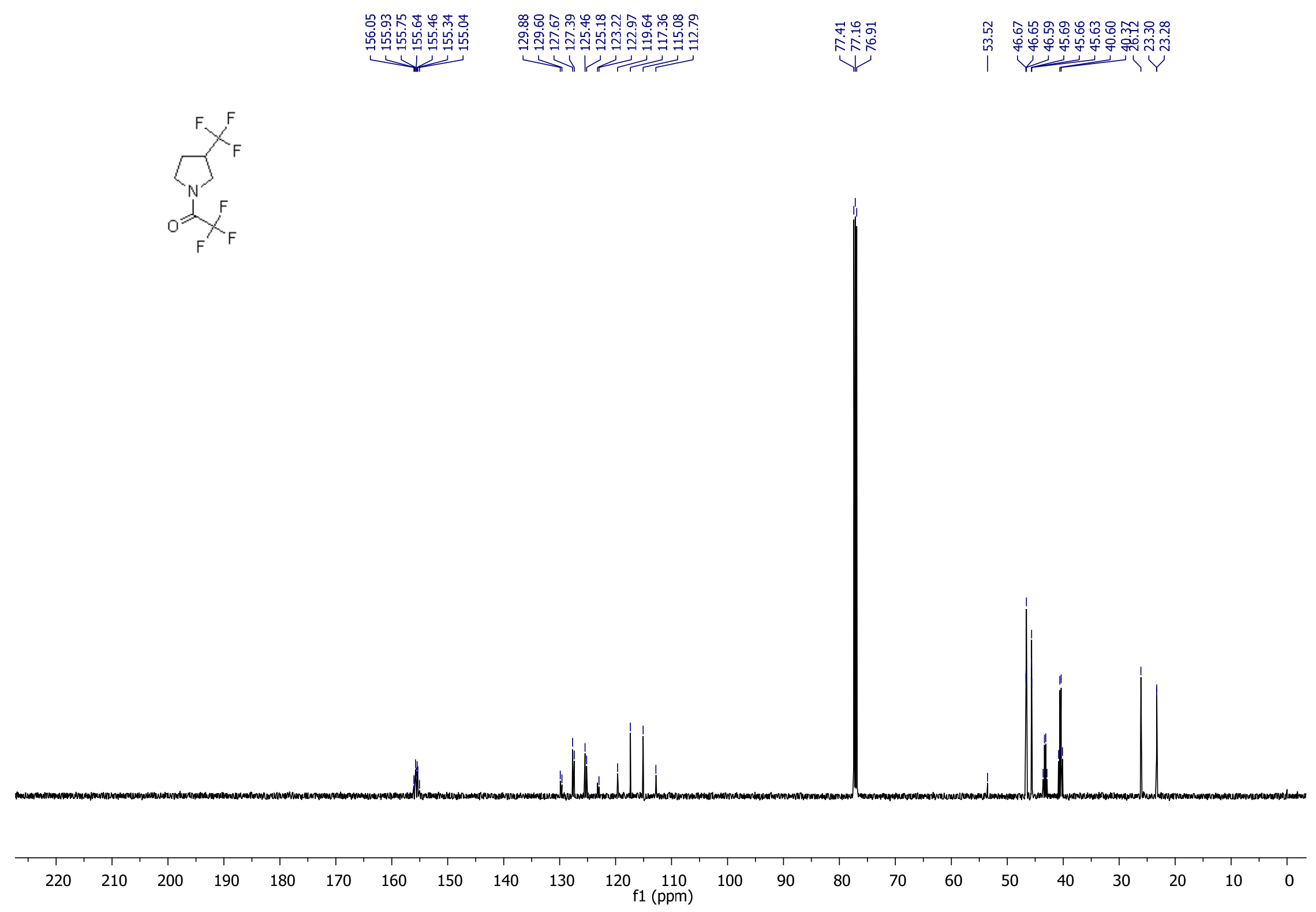




$$
\sum_{F_{F}}^{F_{F}^{F}}
$$

.

$-20$

$-40$

$-60$

-80
$f 1(p p m)$ 


\section{Compound 31a}

igrk-3596

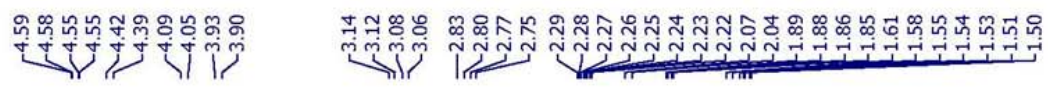
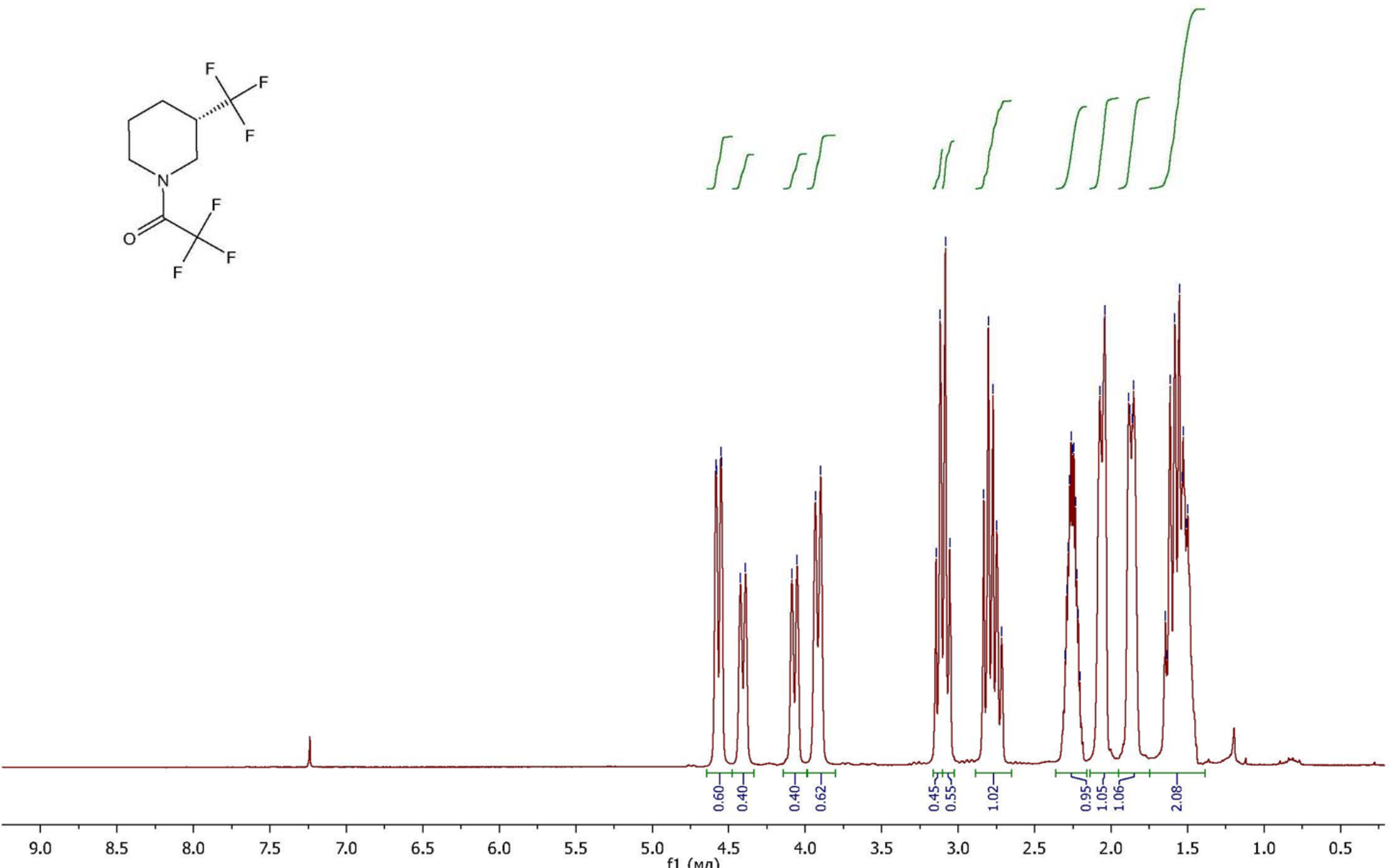


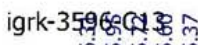

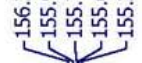

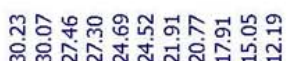

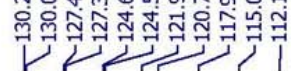

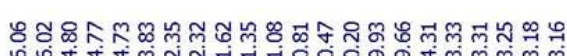

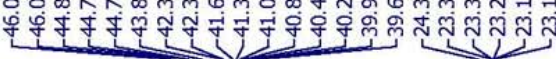<smiles>O=C(N1CCC[C@H](C(F)(F)F)C1)C(F)(F)F</smiles>

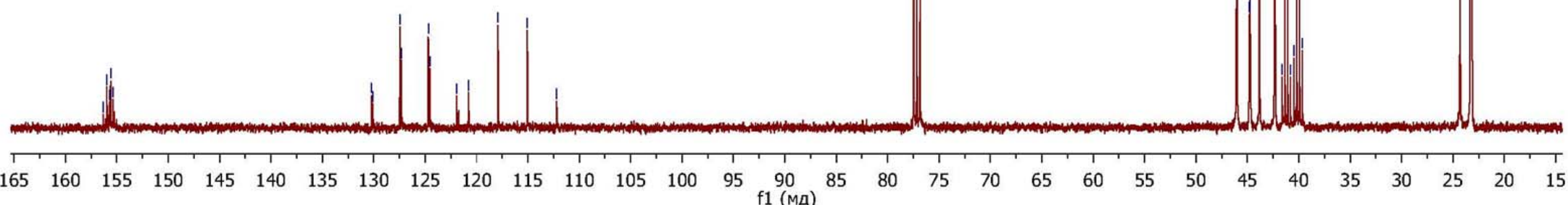




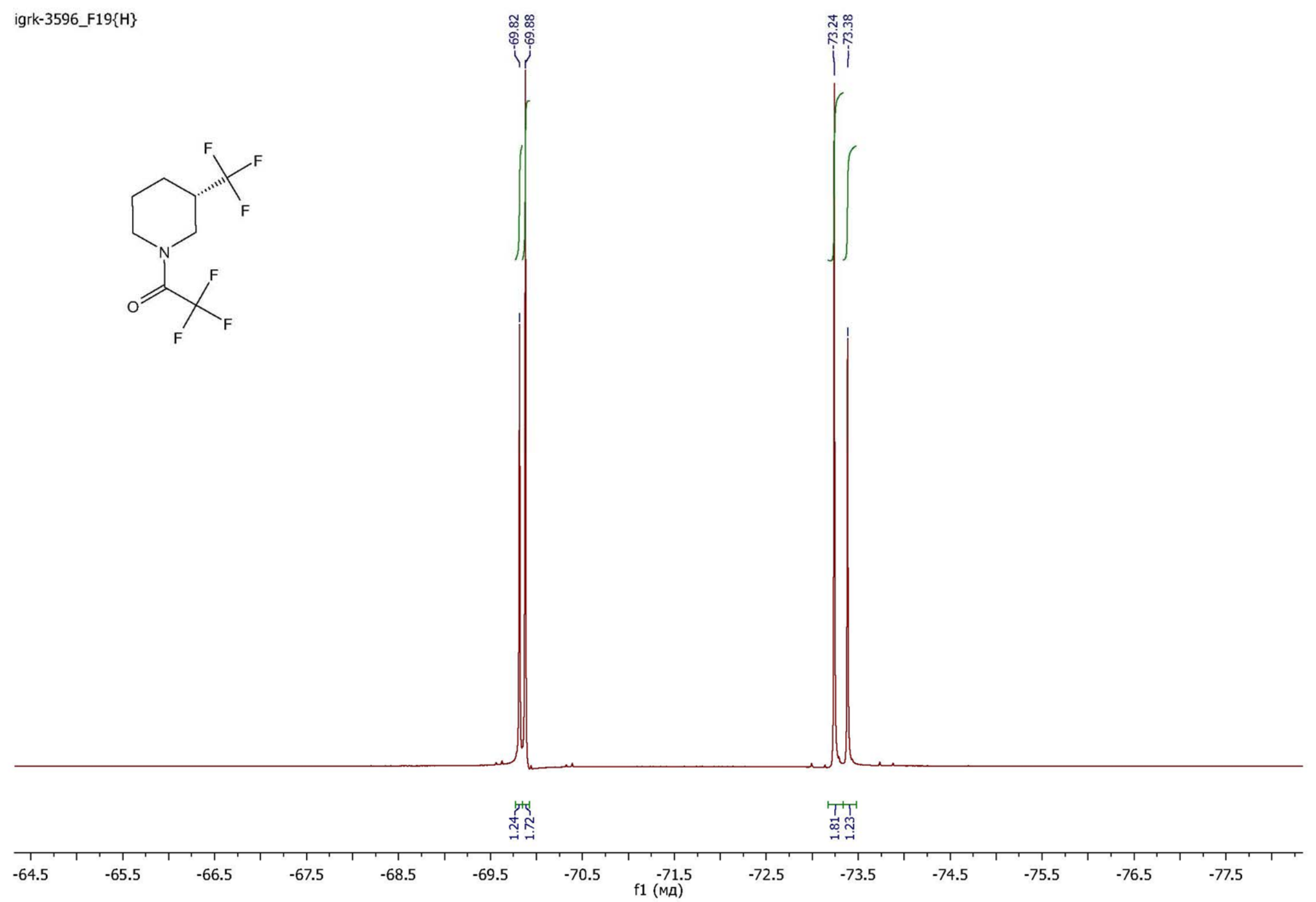




\section{Compound 32a}

igm 11020

$$
\nu_{\mathrm{CF}_{3}}^{\mathrm{CO}_{2} \mathrm{Et}}
$$
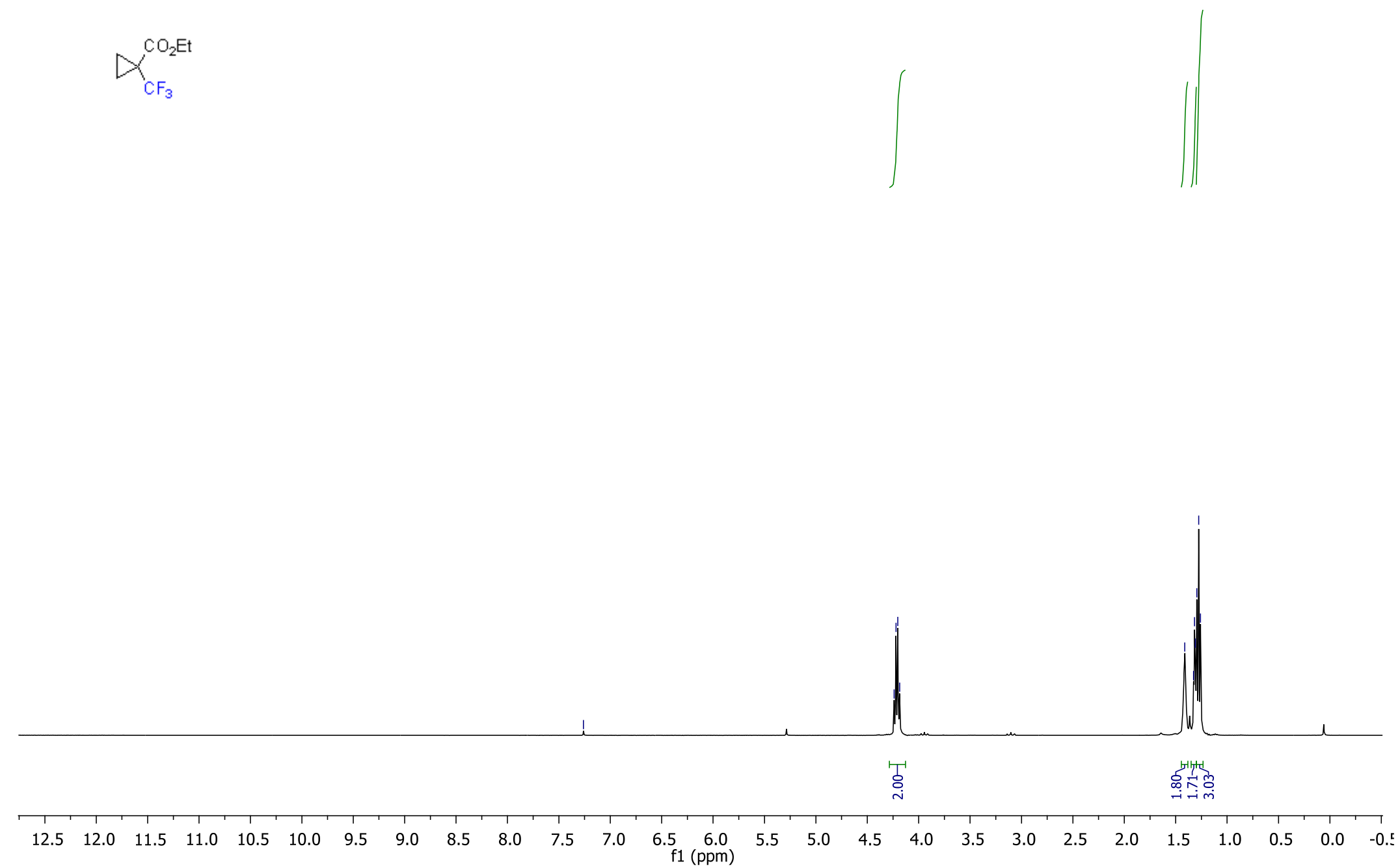
igm11020_C13

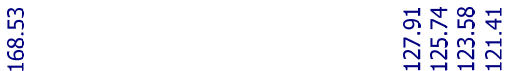

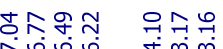

$D_{\mathrm{CF}_{3}}^{\mathrm{CO}_{2} \mathrm{Et}}$
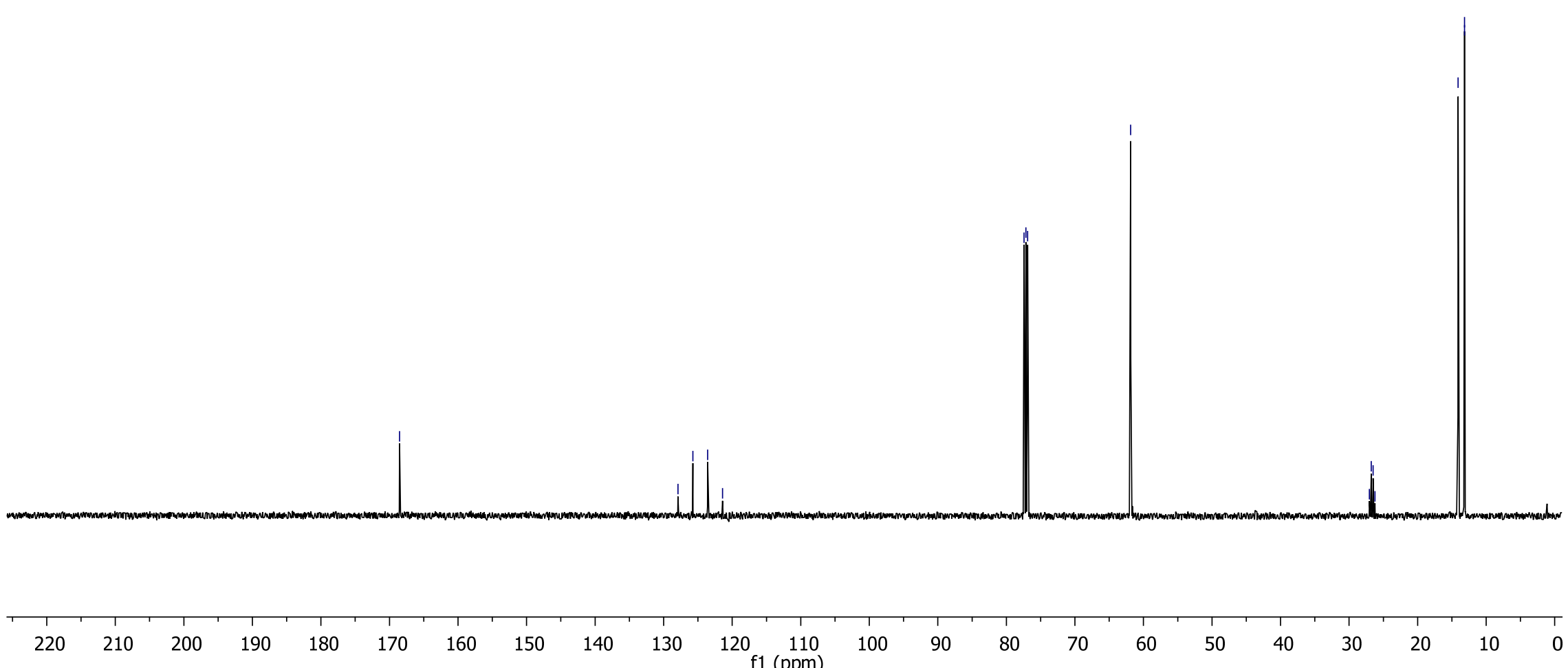
IGm11008_F19

$\aleph_{\mathrm{CF}_{3}}^{\mathrm{CO}_{2} \mathrm{Et}}$

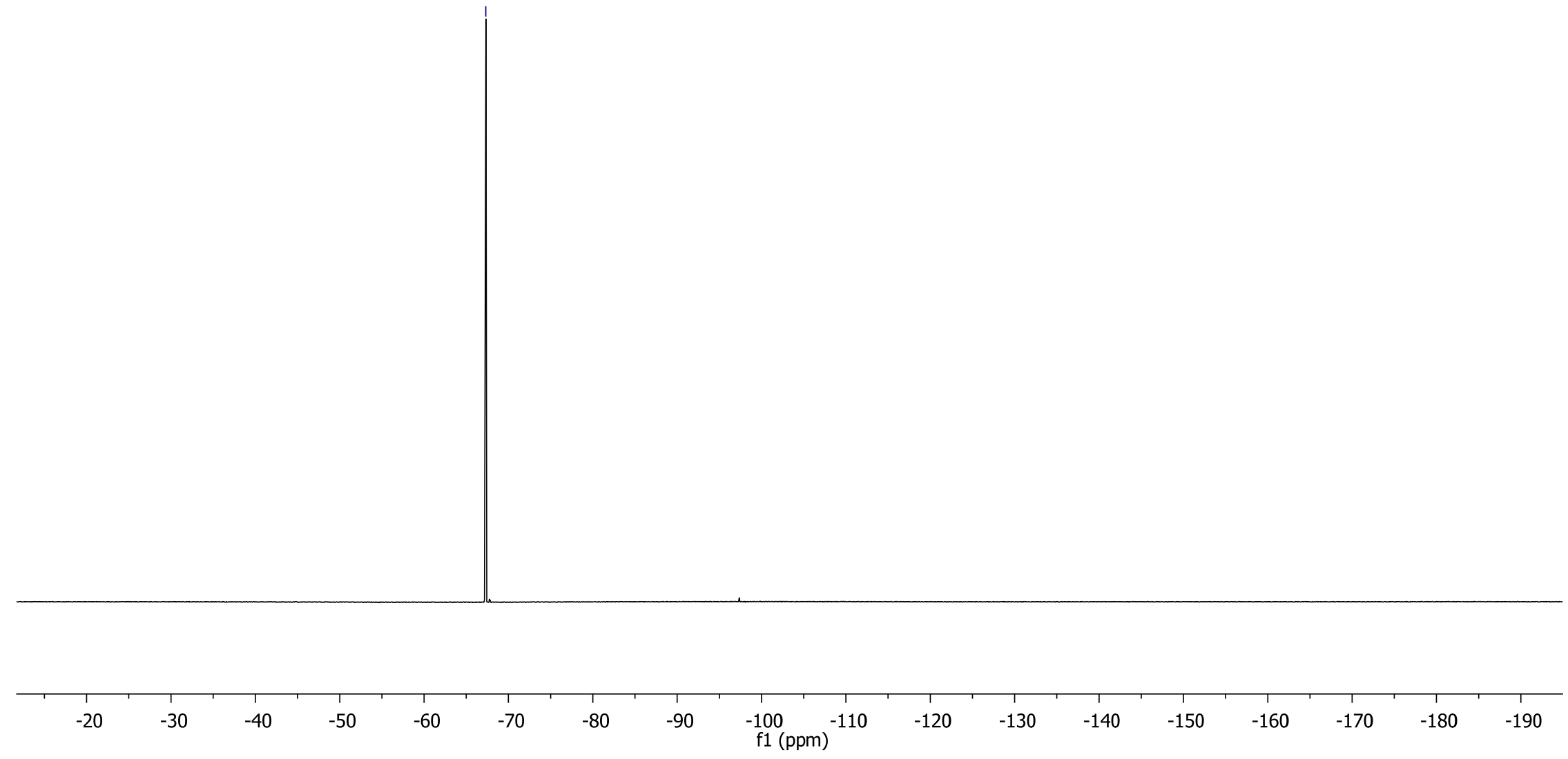




\section{Compound 33a}

R272415

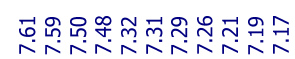

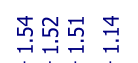<smiles>FC(F)(F)C1(c2ccccc2Br)CC1</smiles>
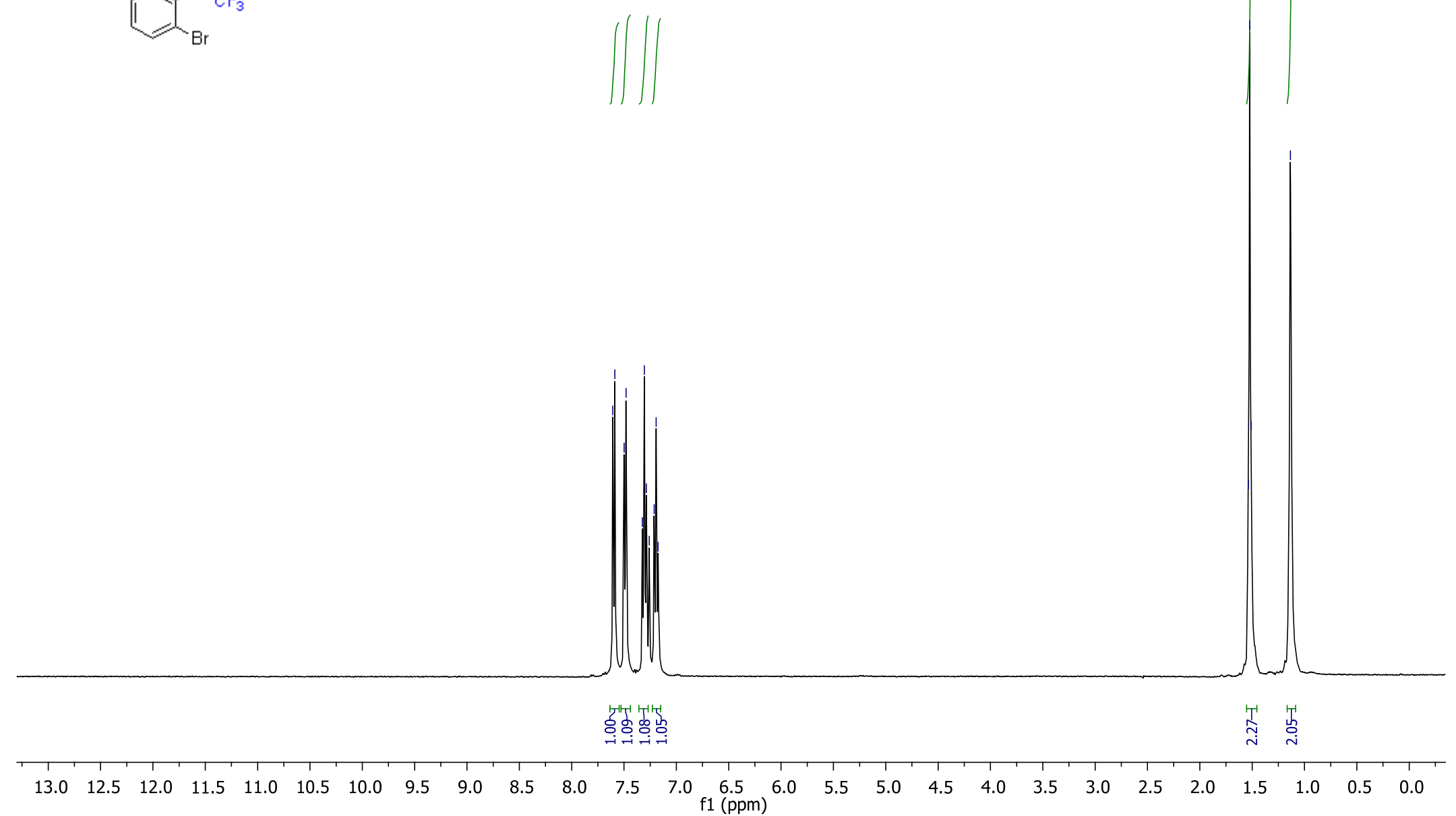


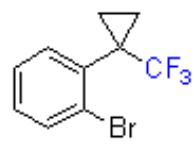

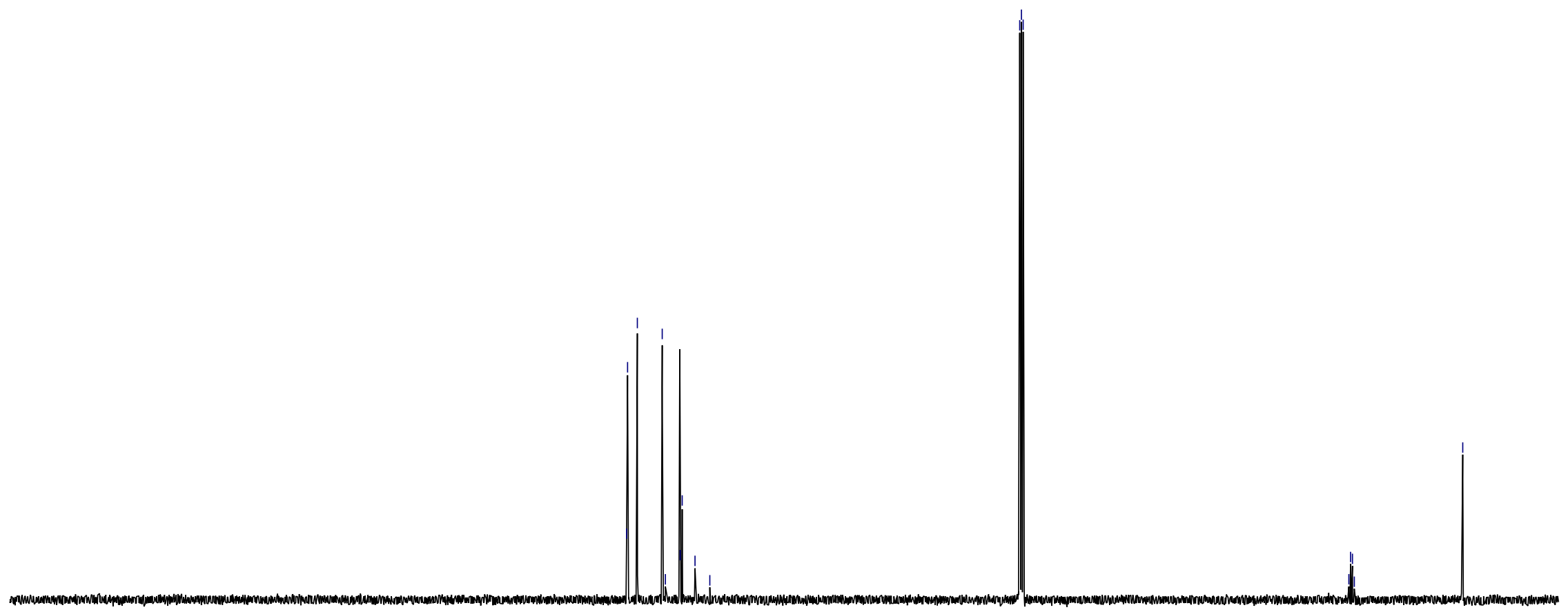



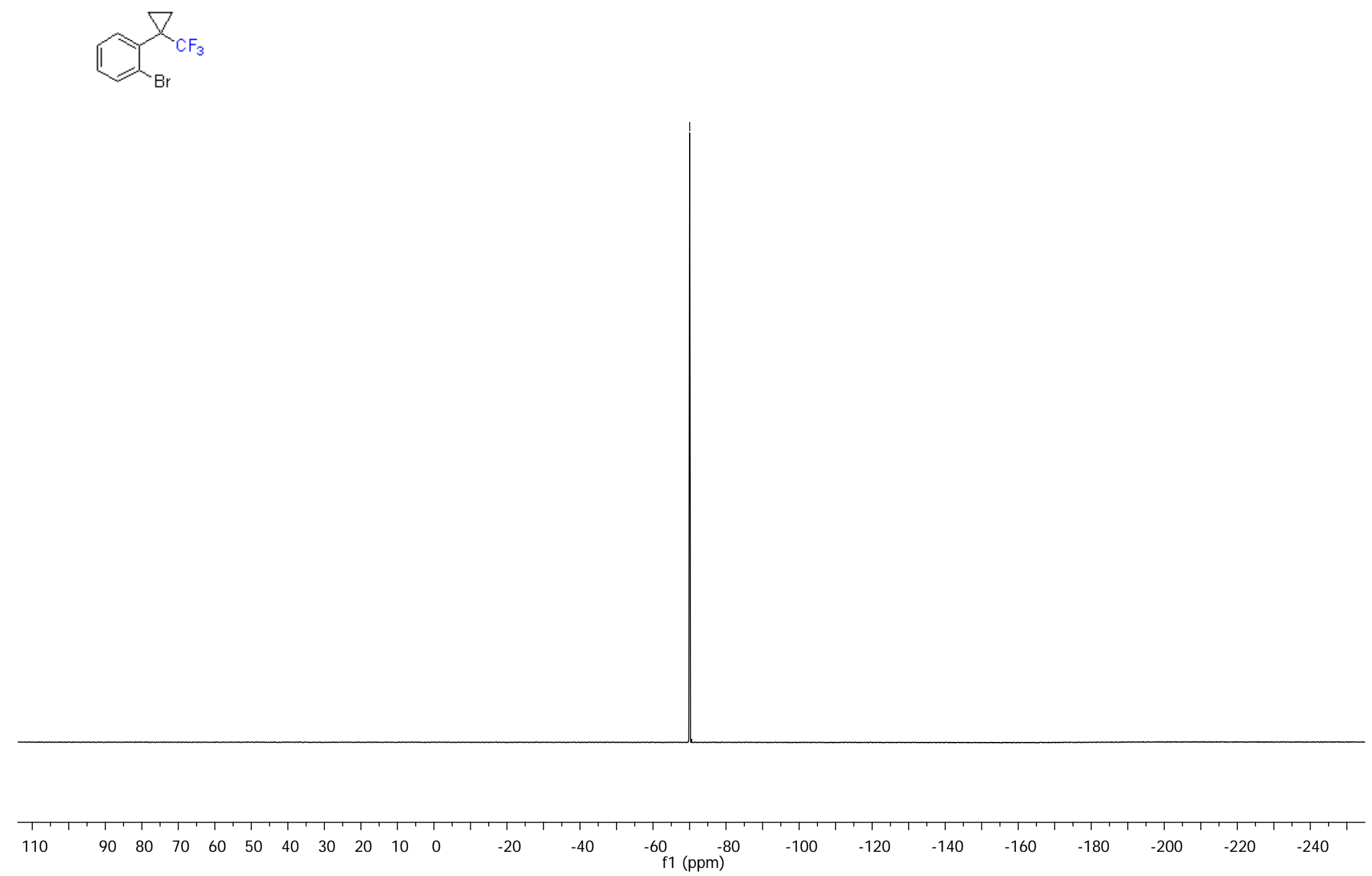


\section{Compound 34a}

R192041

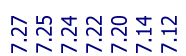

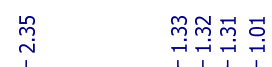

$\gamma_{\mathrm{CF}_{3}}$
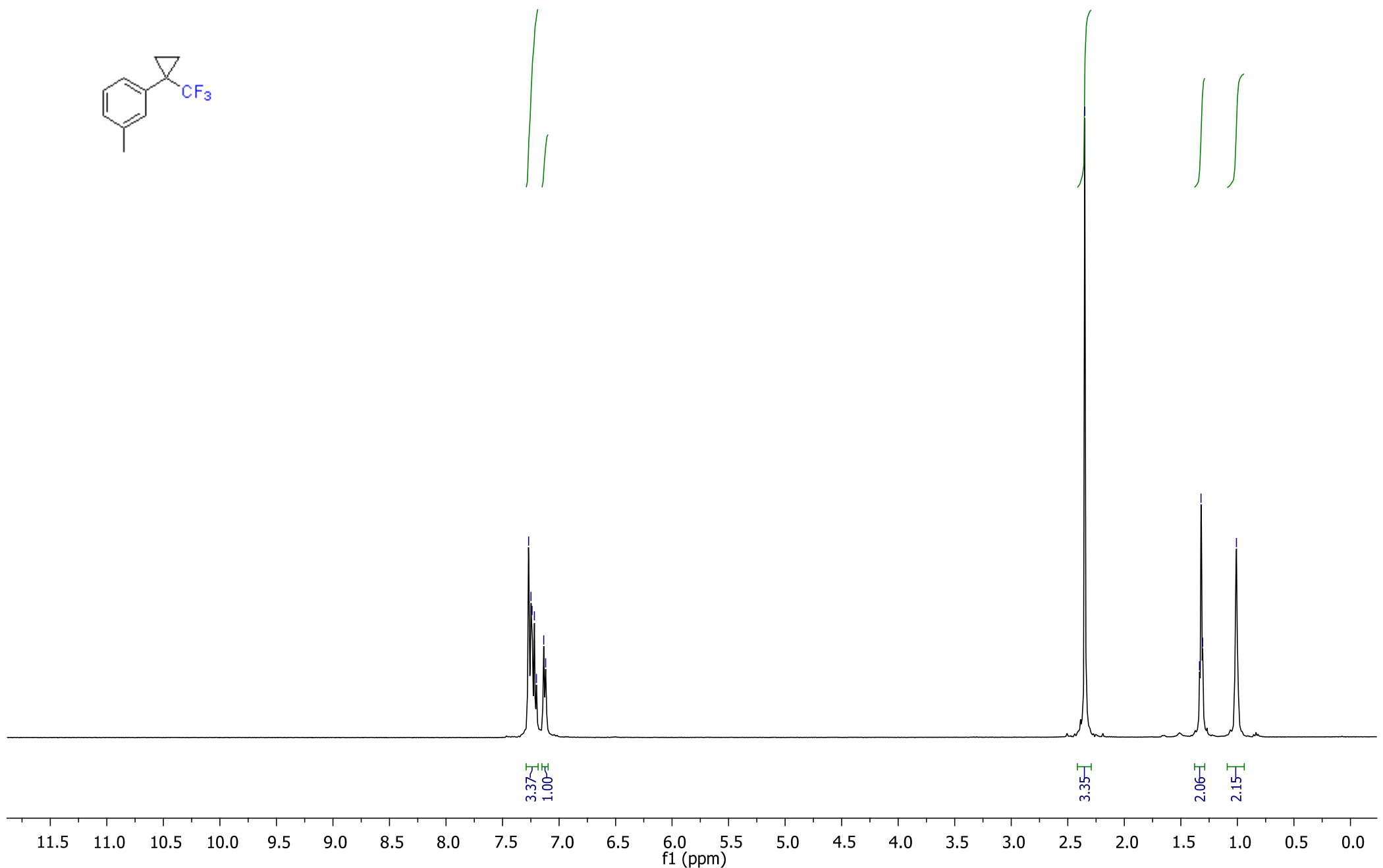

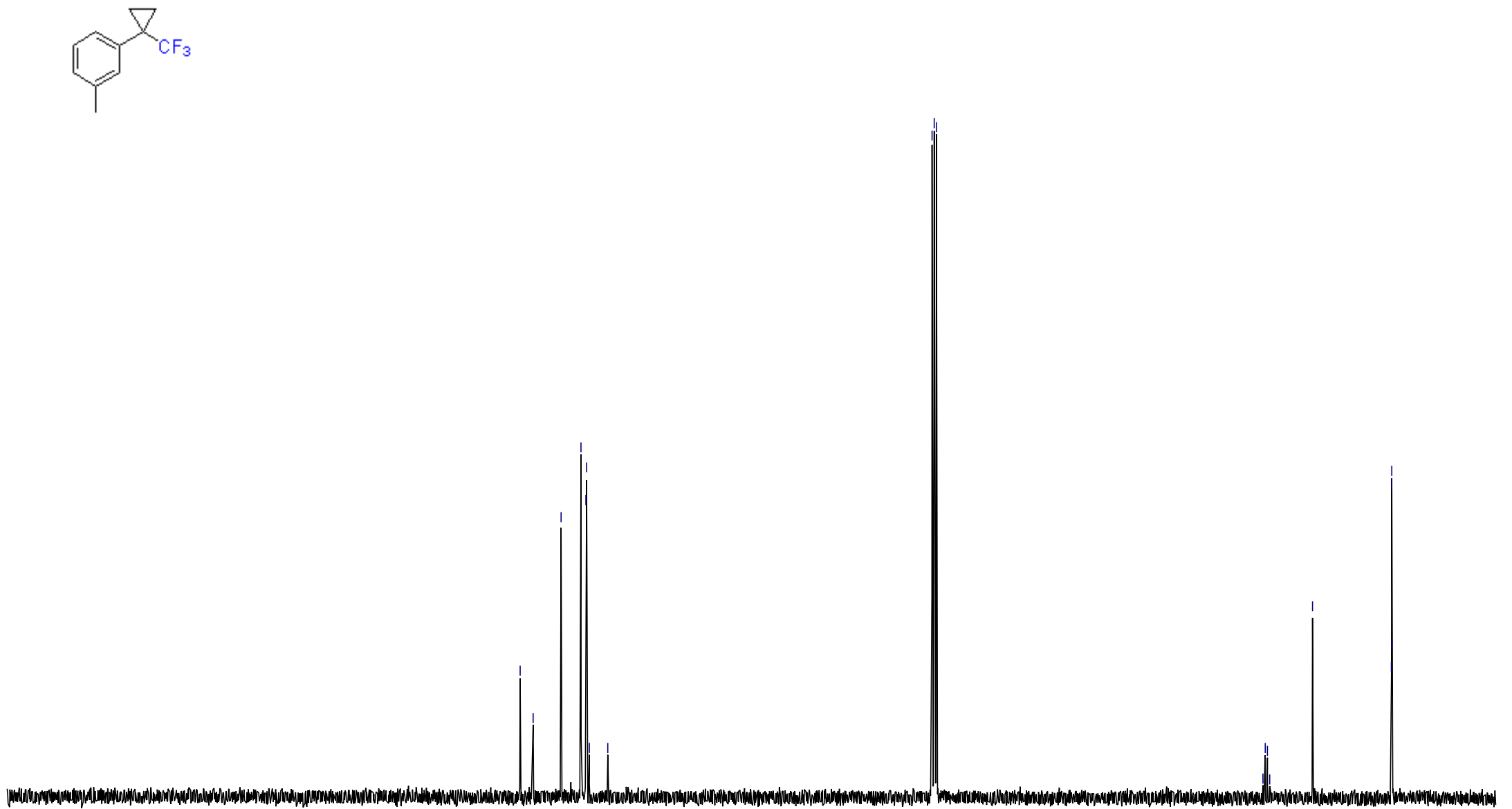
R192041_F19 $\{\mathrm{H}\}$ $19 \mathrm{~F}-\{1 \mathrm{H}\}$

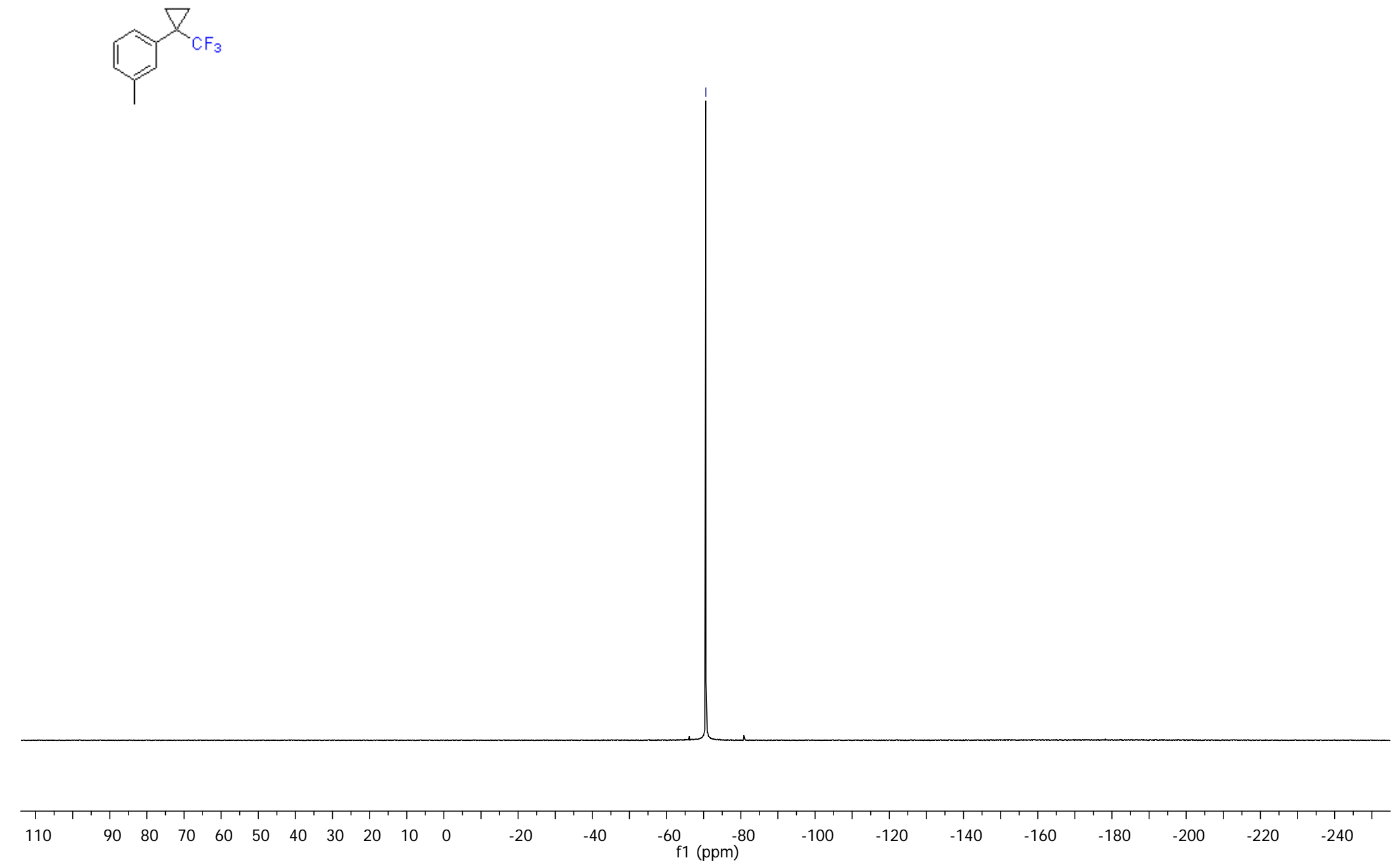




\section{Compound 35a}

am647

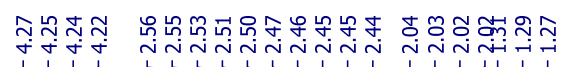

$\Delta \mathrm{CF}_{3}^{\mathrm{CO}_{2} \mathrm{Et}}$

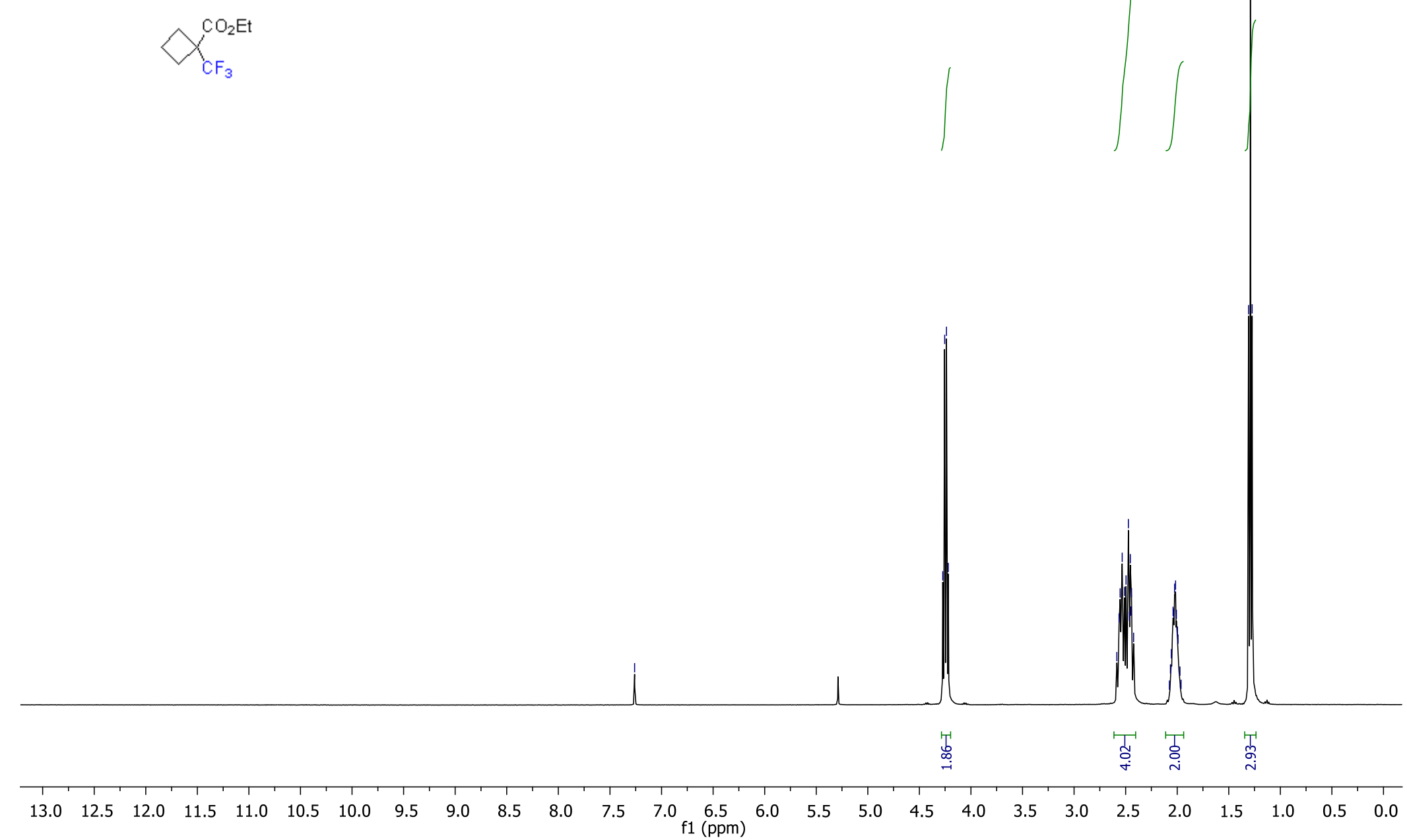


sb683_C13

$\Delta \mathrm{CF}_{\mathrm{F}_{3}}^{\mathrm{CO}_{2} \mathrm{Et}}$

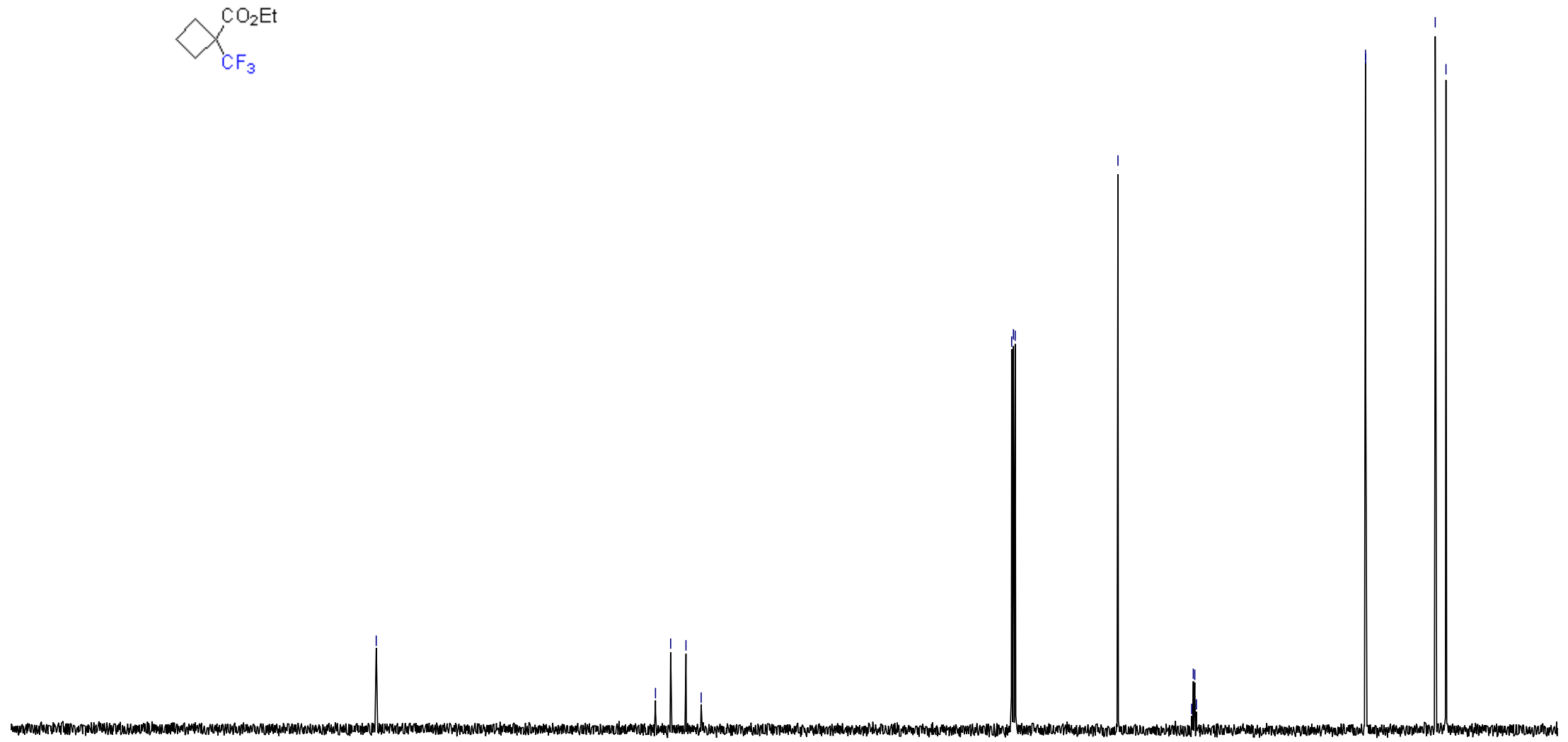


sb694_F19

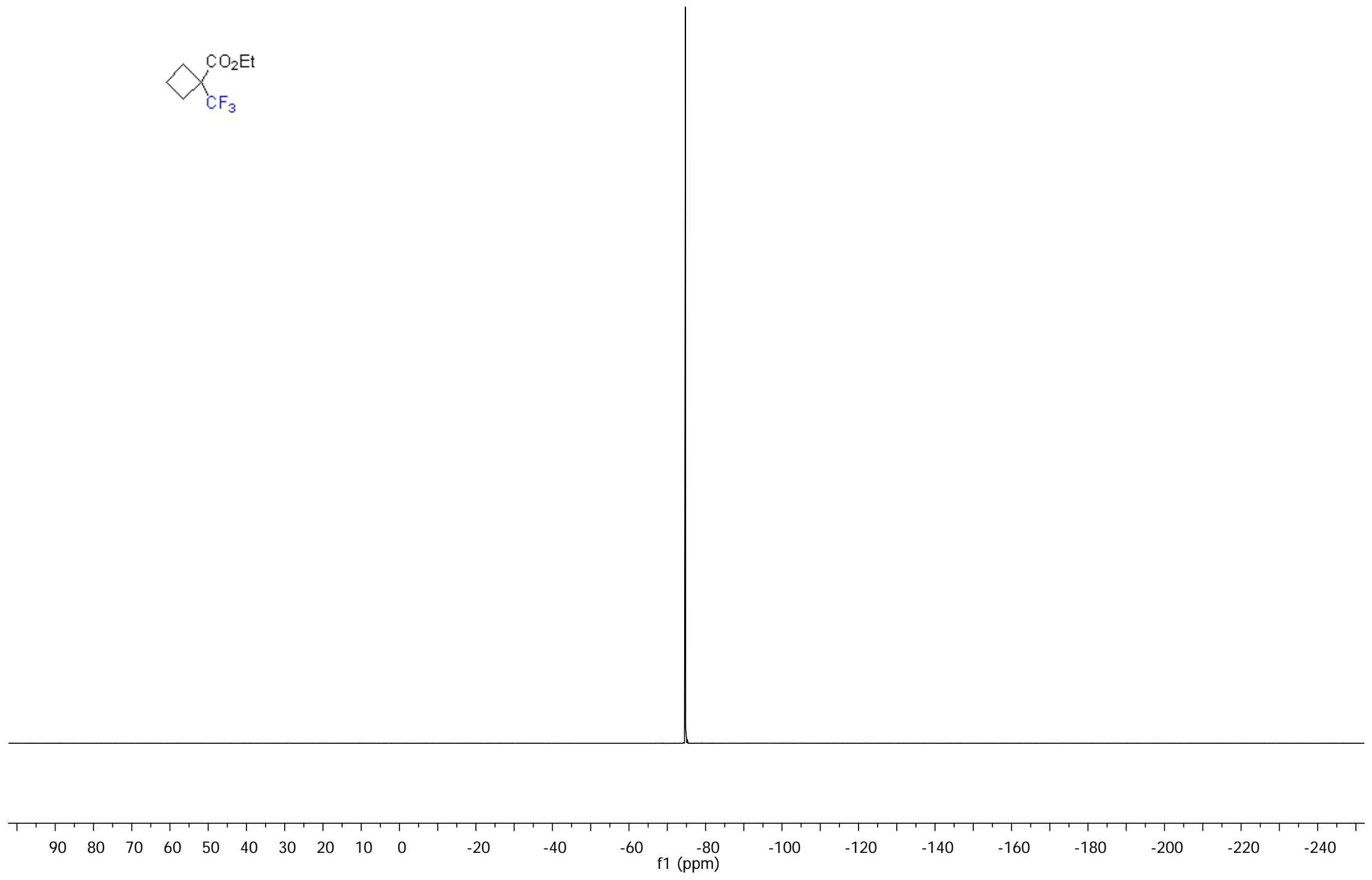




\section{Compound 36a}

igmn44<smiles>CCOC1(C)CCC2(CCCC2)C1</smiles>
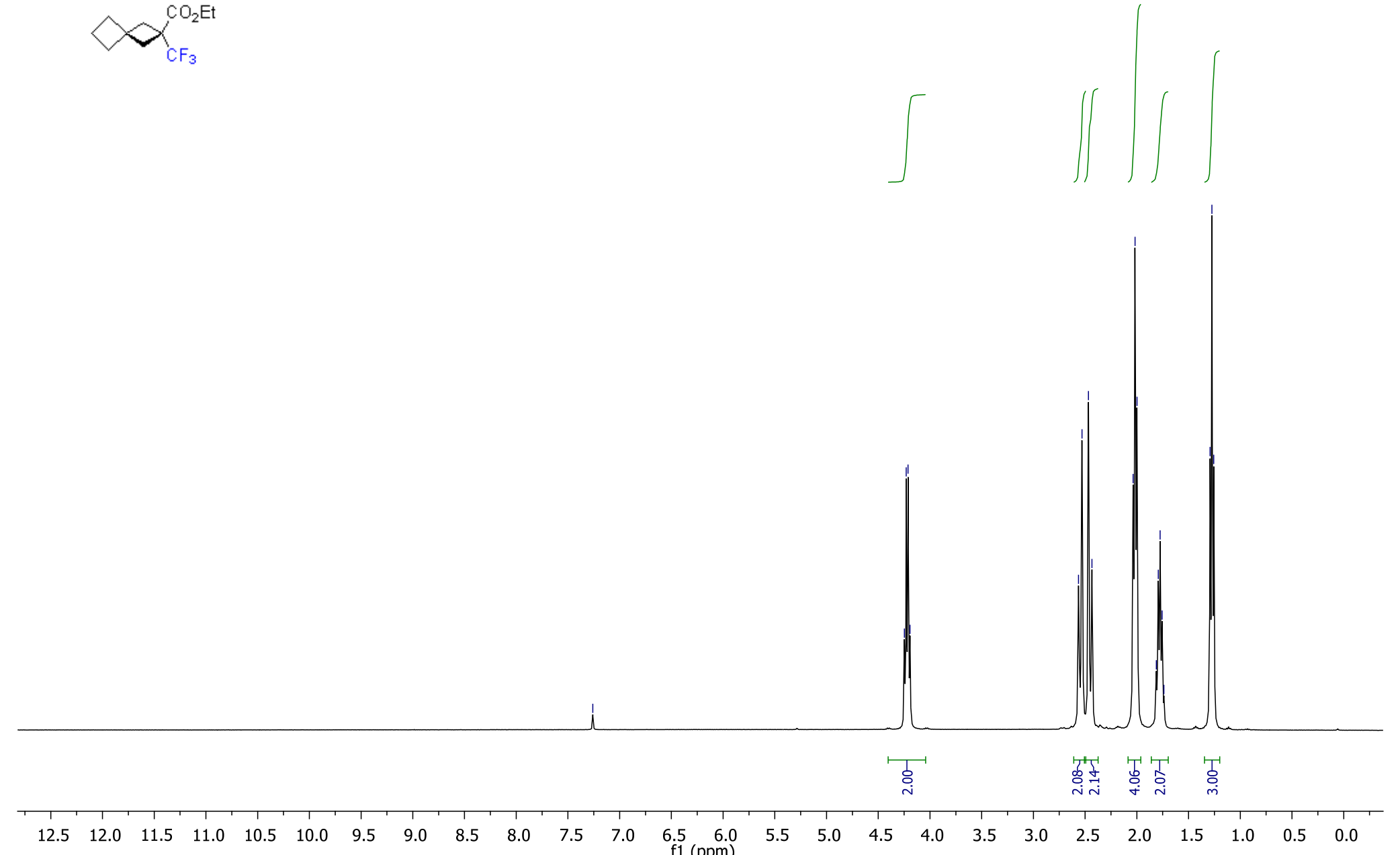
igmn44_C13

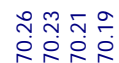

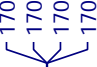

둥화요

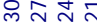

| | |

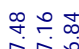

i

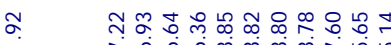

子掟

(1)

象

| 1

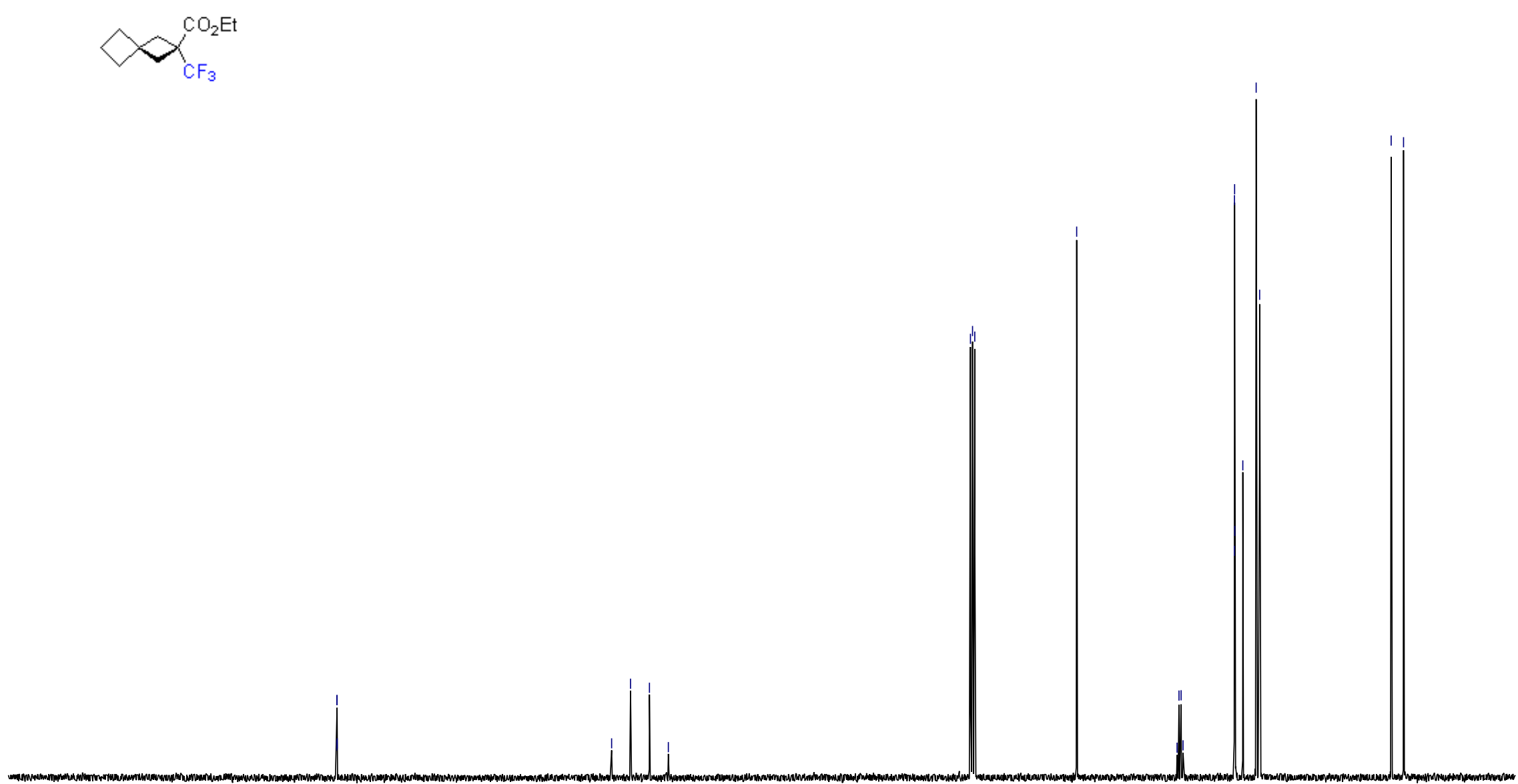

210 

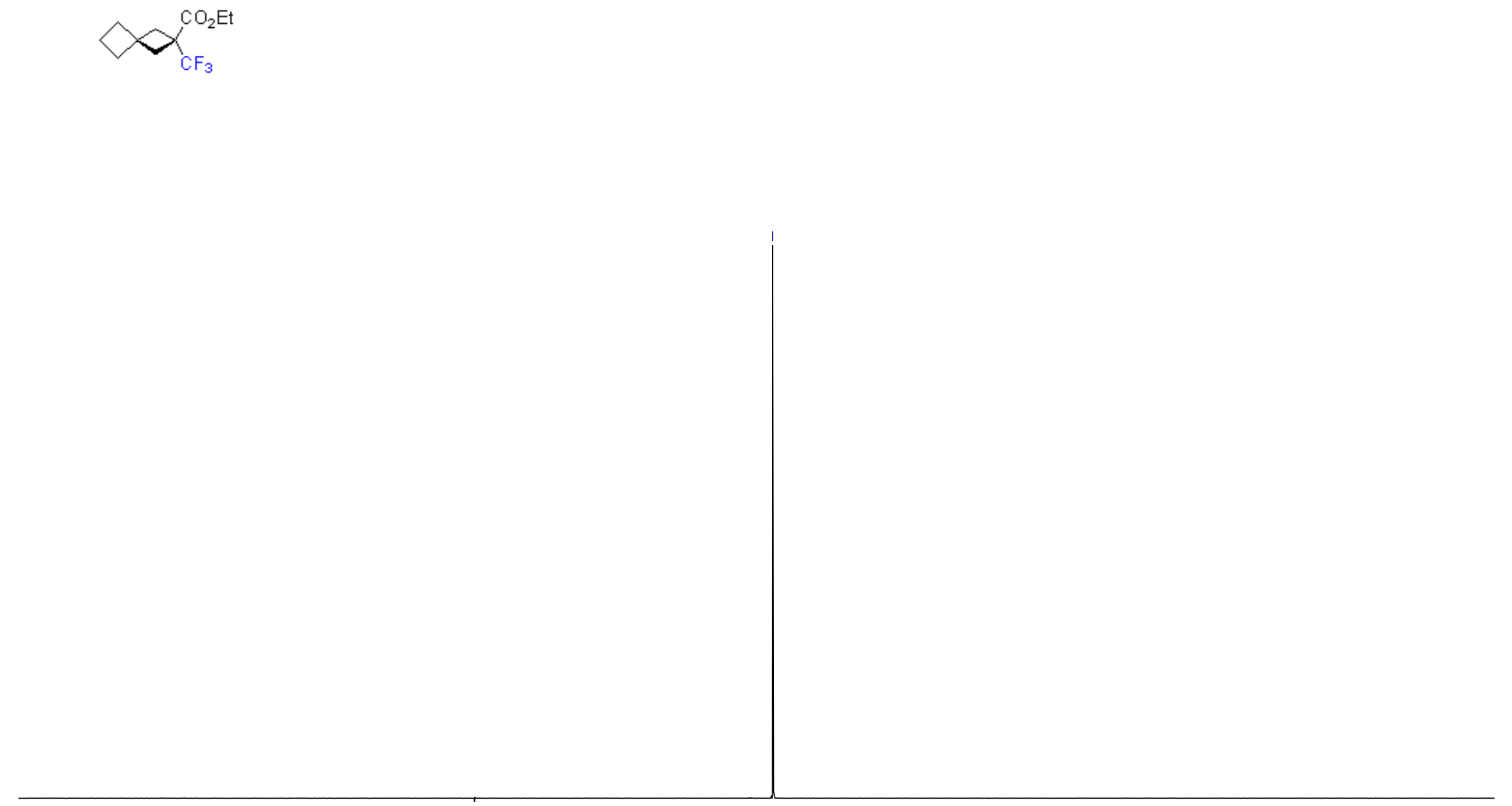

$\begin{array}{llllllllllllllllllllllll} & 90 & 80 & 70 & 60 & 50 & 40 & 30 & 20 & 10 & 0 & -20 & -40 & -60 & -80 & -100 & -120 & -140 & -160 & -180 & -200 & -220 & -240 & \end{array}$




\section{Compound 37a}

R1250036
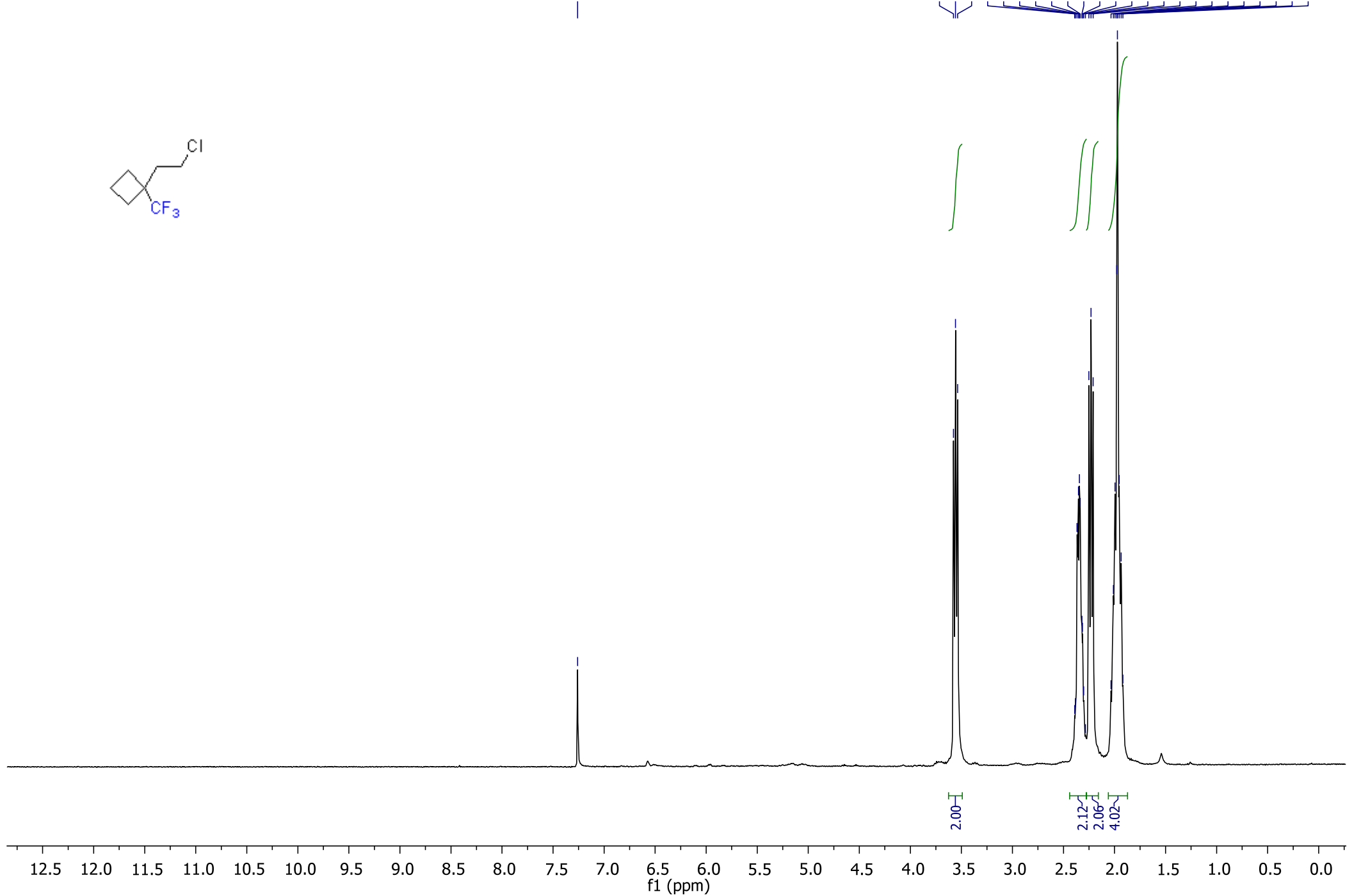


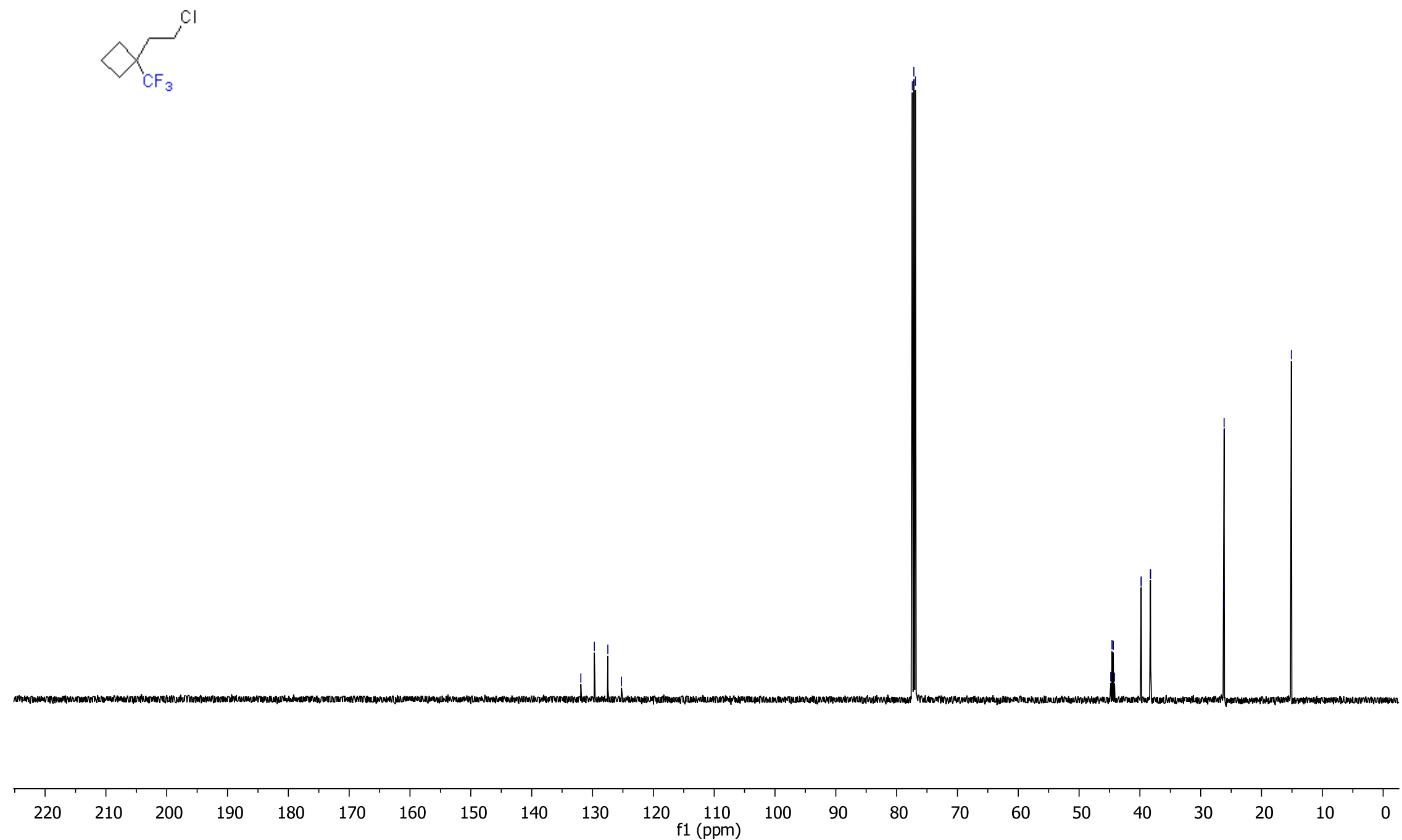


R1250036_F19 $\{\mathrm{H}\}$
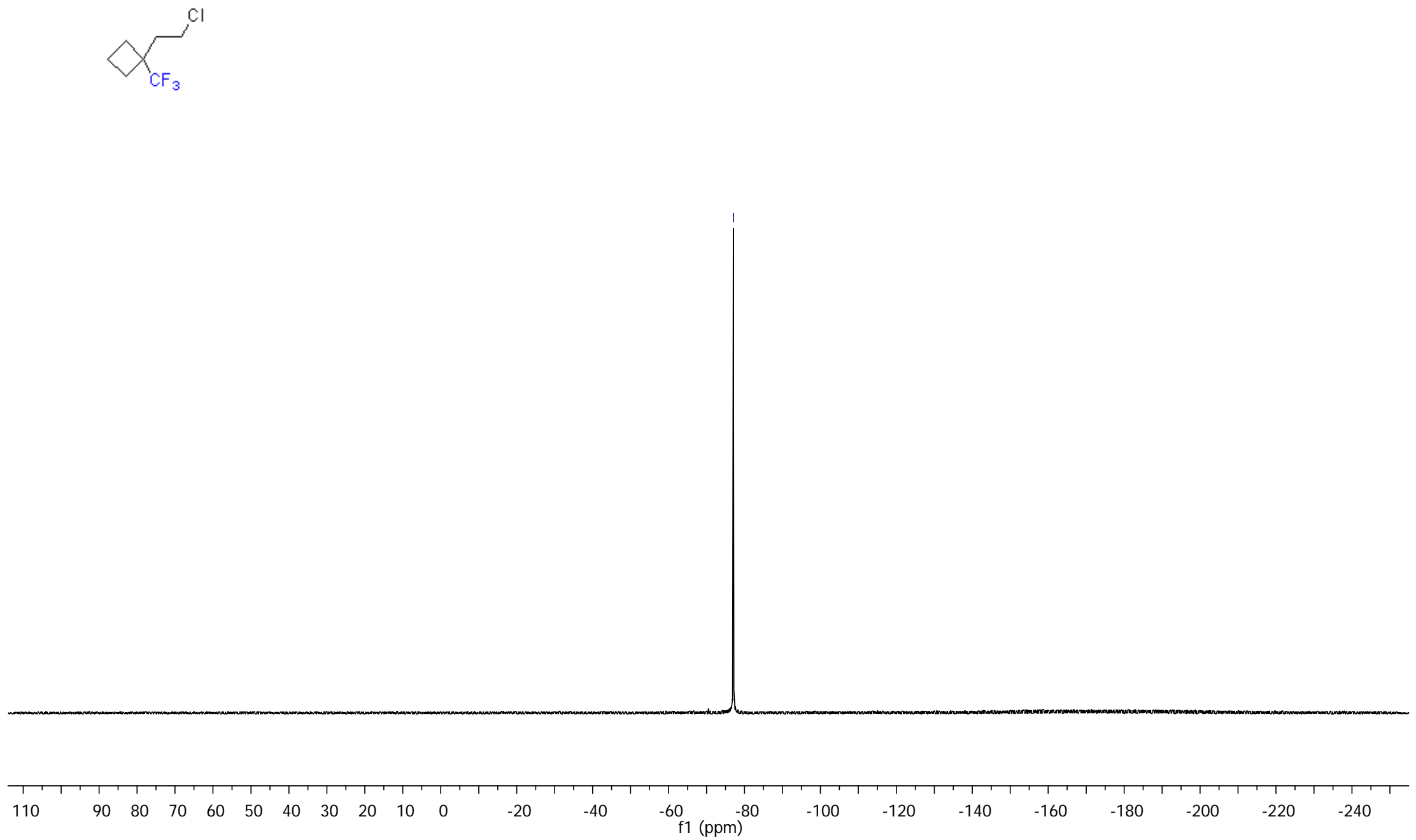


\section{Compound 38a}

igm11021

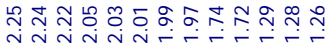

$\mathrm{C}_{\mathrm{CF}_{3}}^{\mathrm{CO}_{2} \mathrm{Et}}$
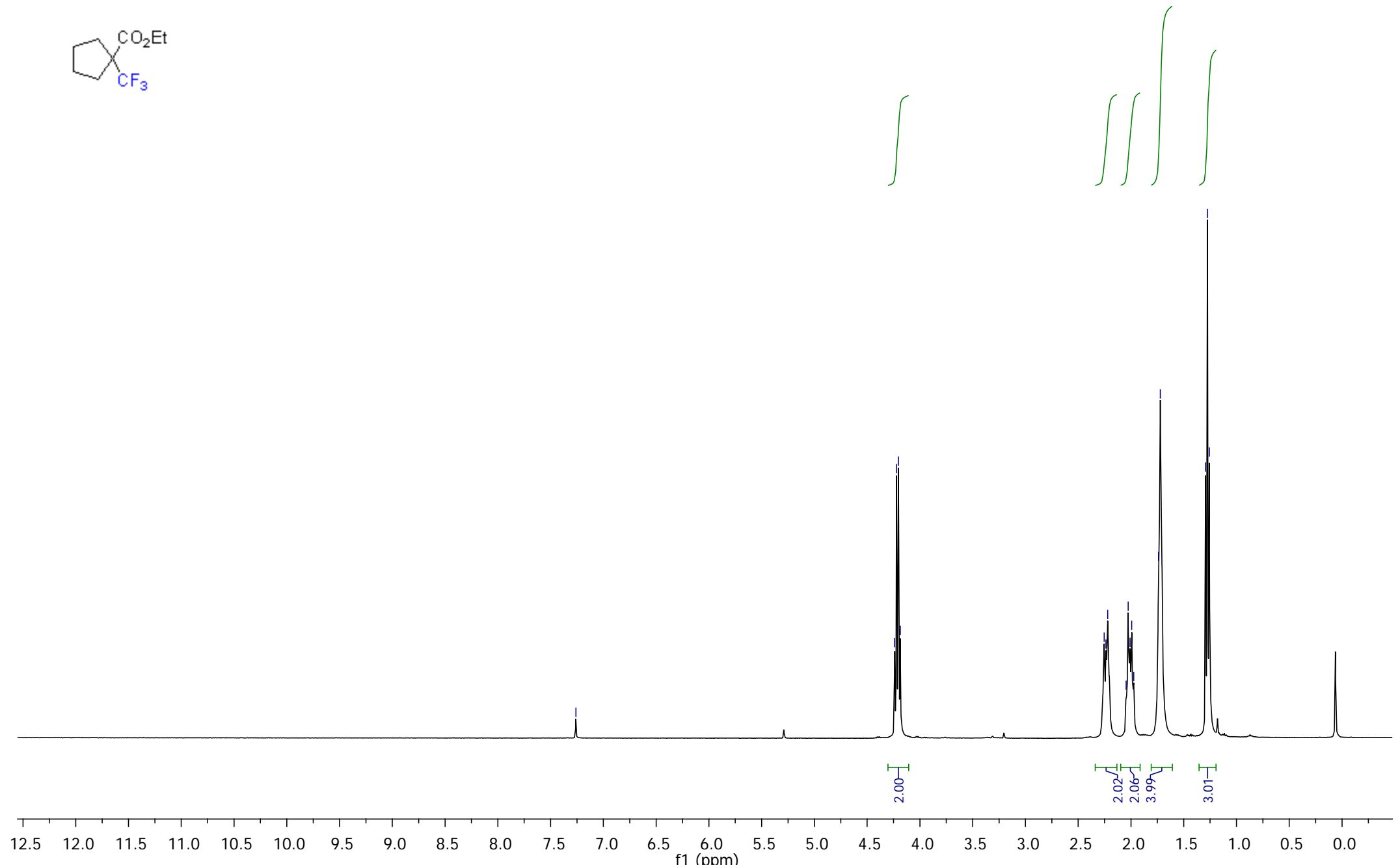
igm11021_C13

$\mathrm{C}_{\mathrm{CF}_{3}}^{\mathrm{CO}_{2} \mathrm{Et}}$

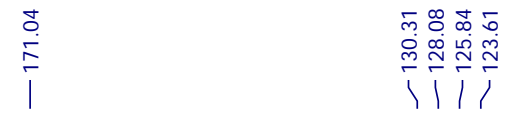

|

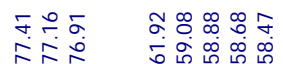

$\underbrace{R}$

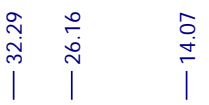

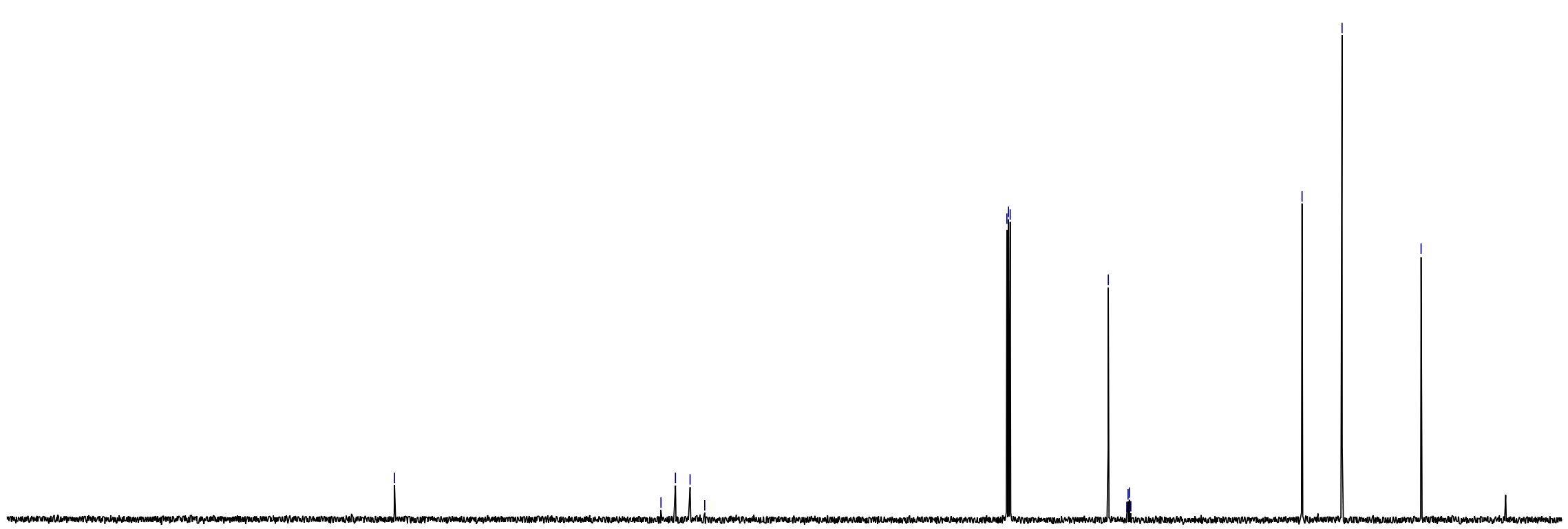

$\begin{array}{llllllllllllllllllllllllllllllllllllll}230 & 220 & 210 & 200 & 190 & 180 & 170 & 160 & 150 & 140 & 130 & 120 & 110 & 100 & 90 & 80 & 70 & 60 & 50 & 40 & 30 & 20 & 10 & 0\end{array}$ 


\section{Compound 39a}

tp11380

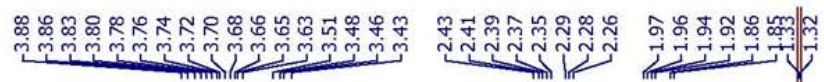
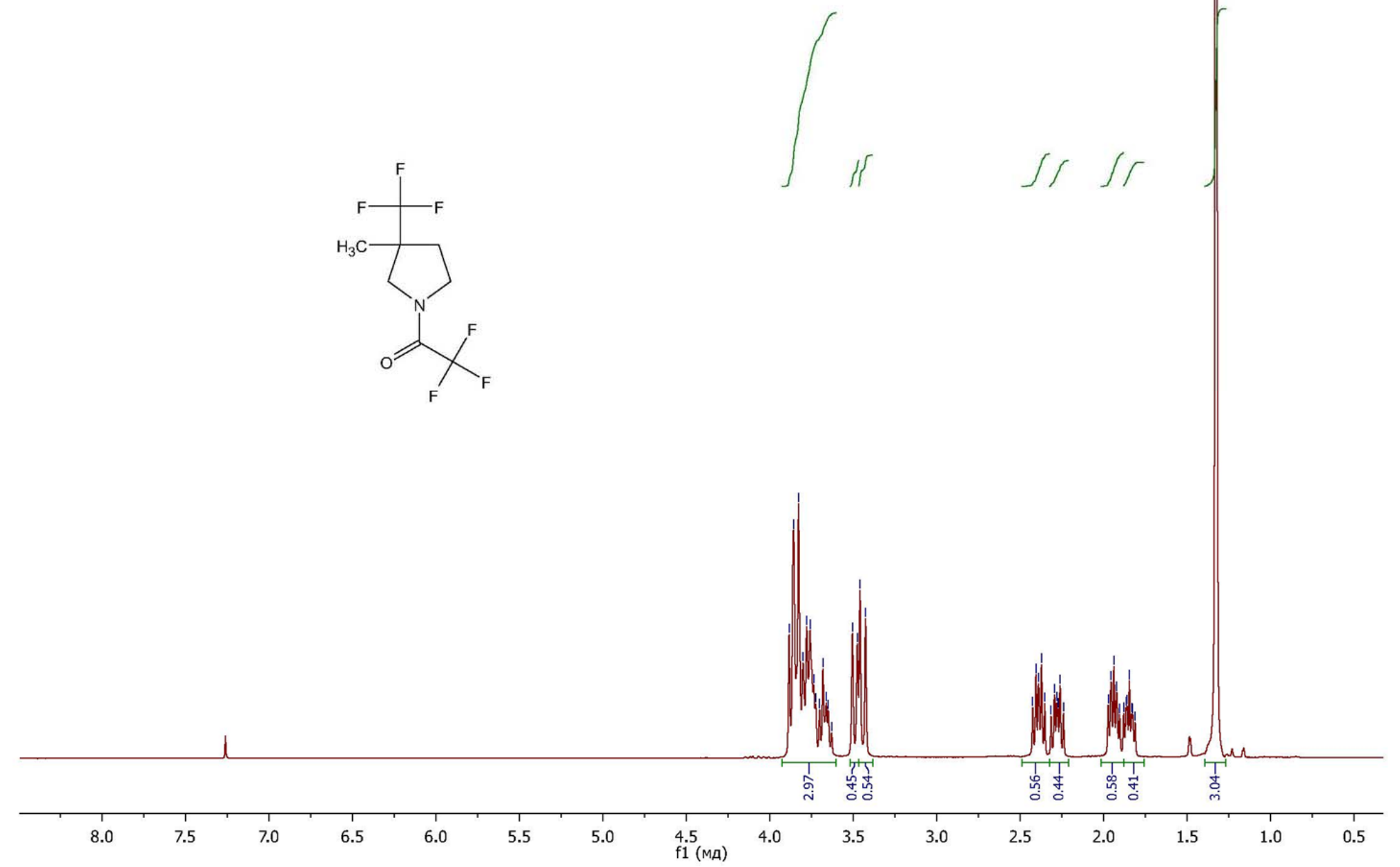
tp

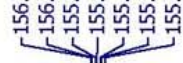

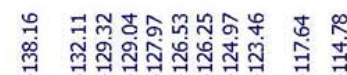

|

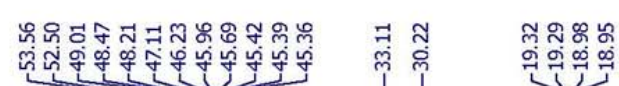

(1) पर
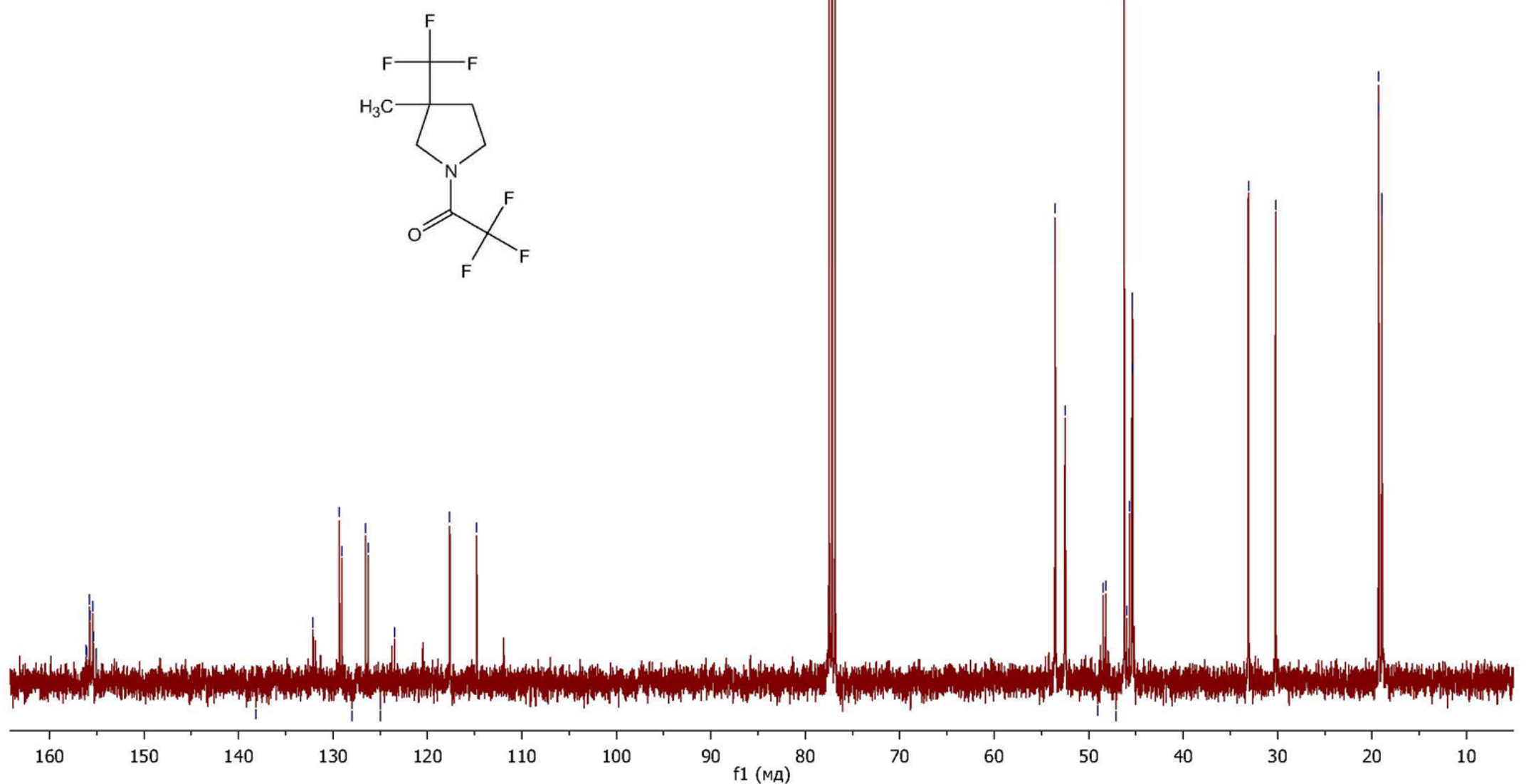


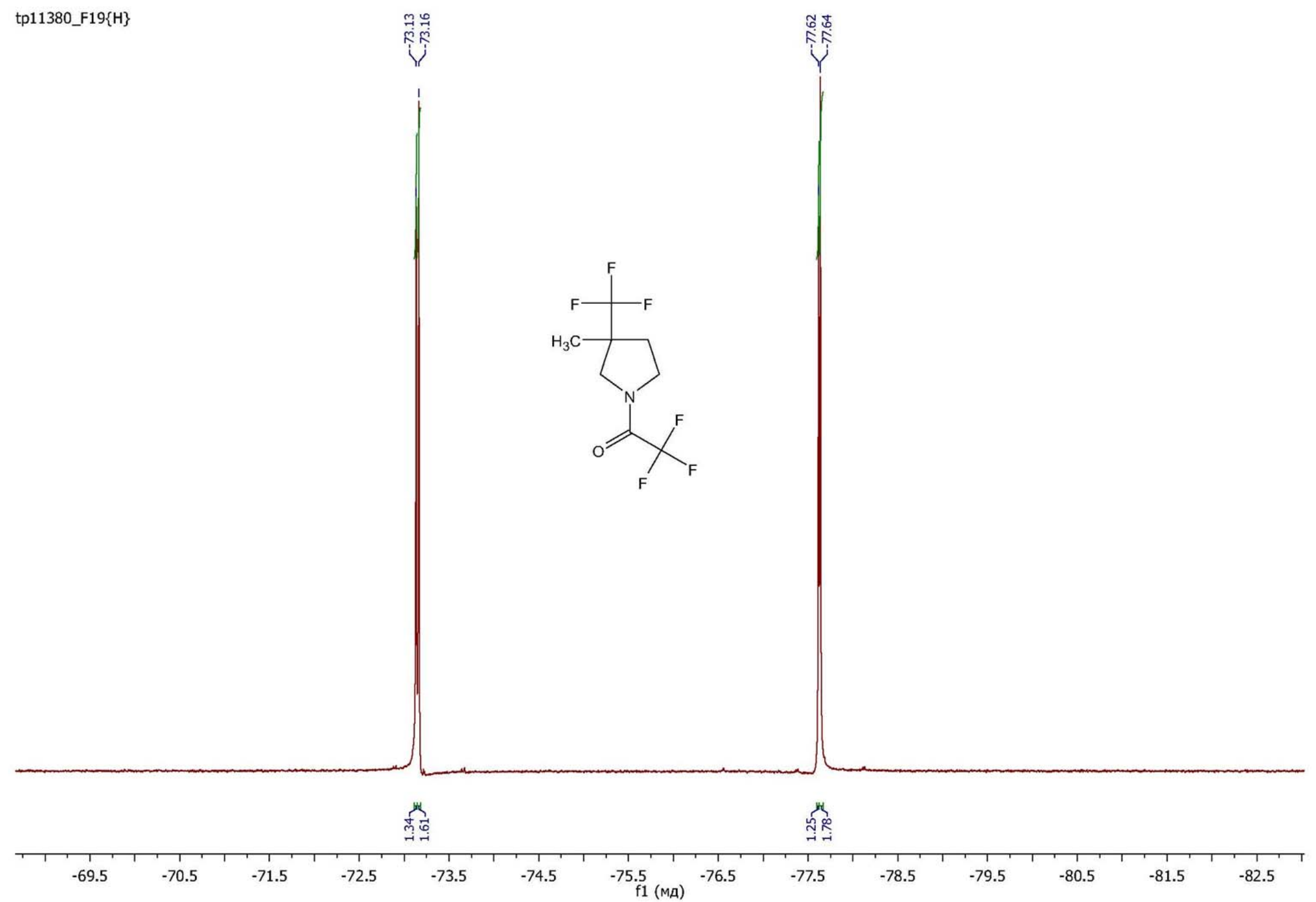




\section{Compound 40a}

tp11381

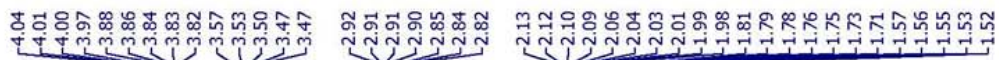
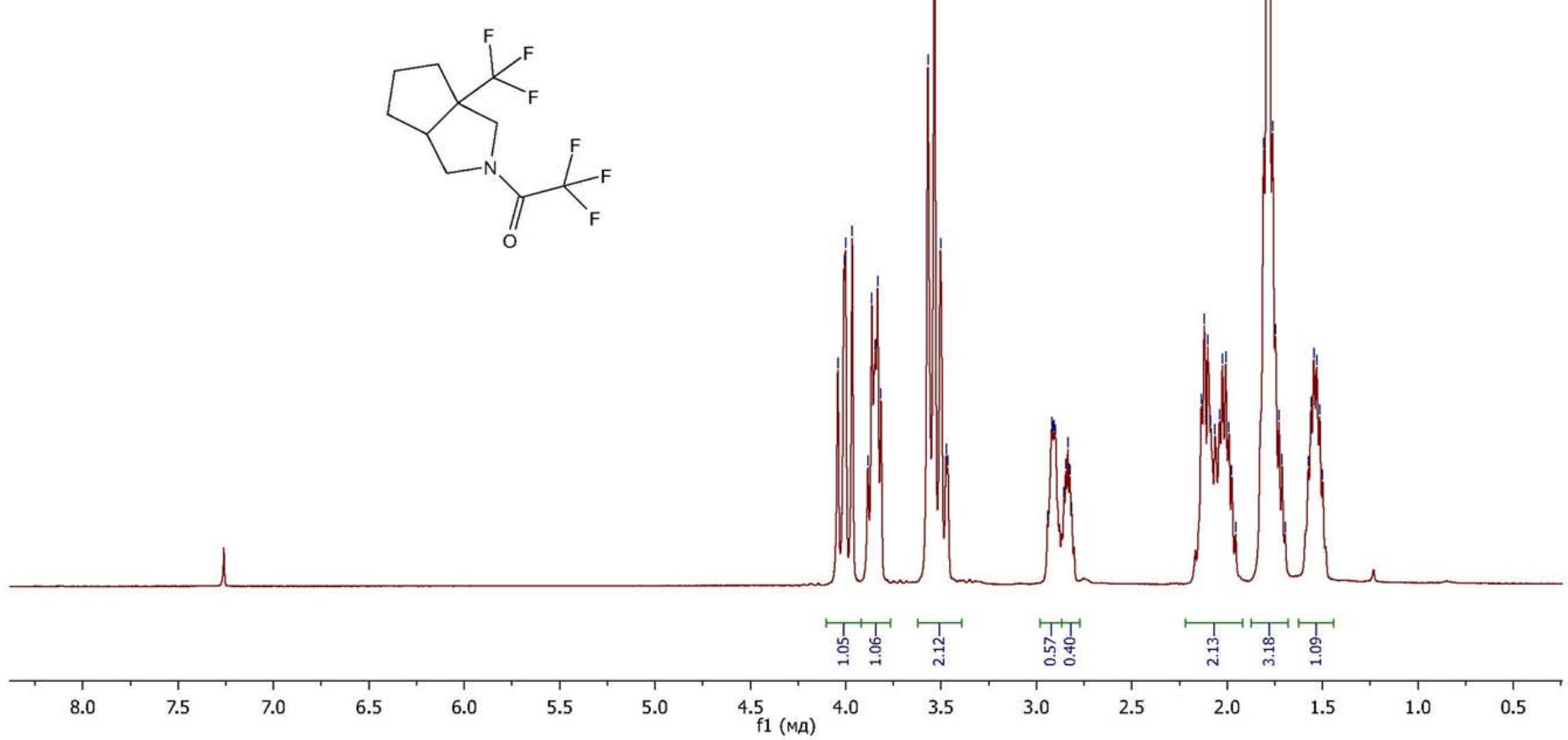
tp11381_C13 ชำํำ

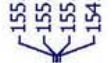

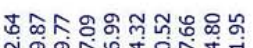

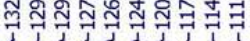

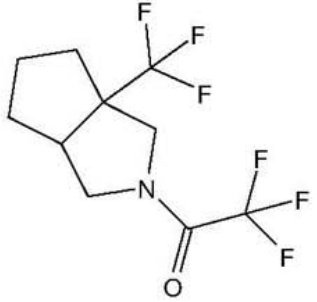

(n)

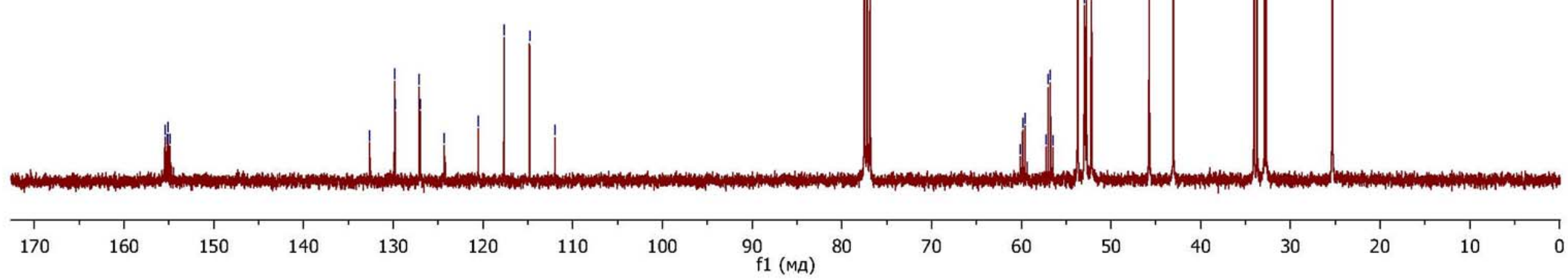

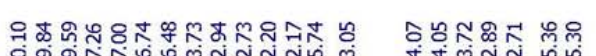

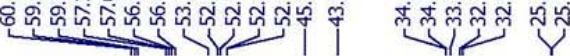




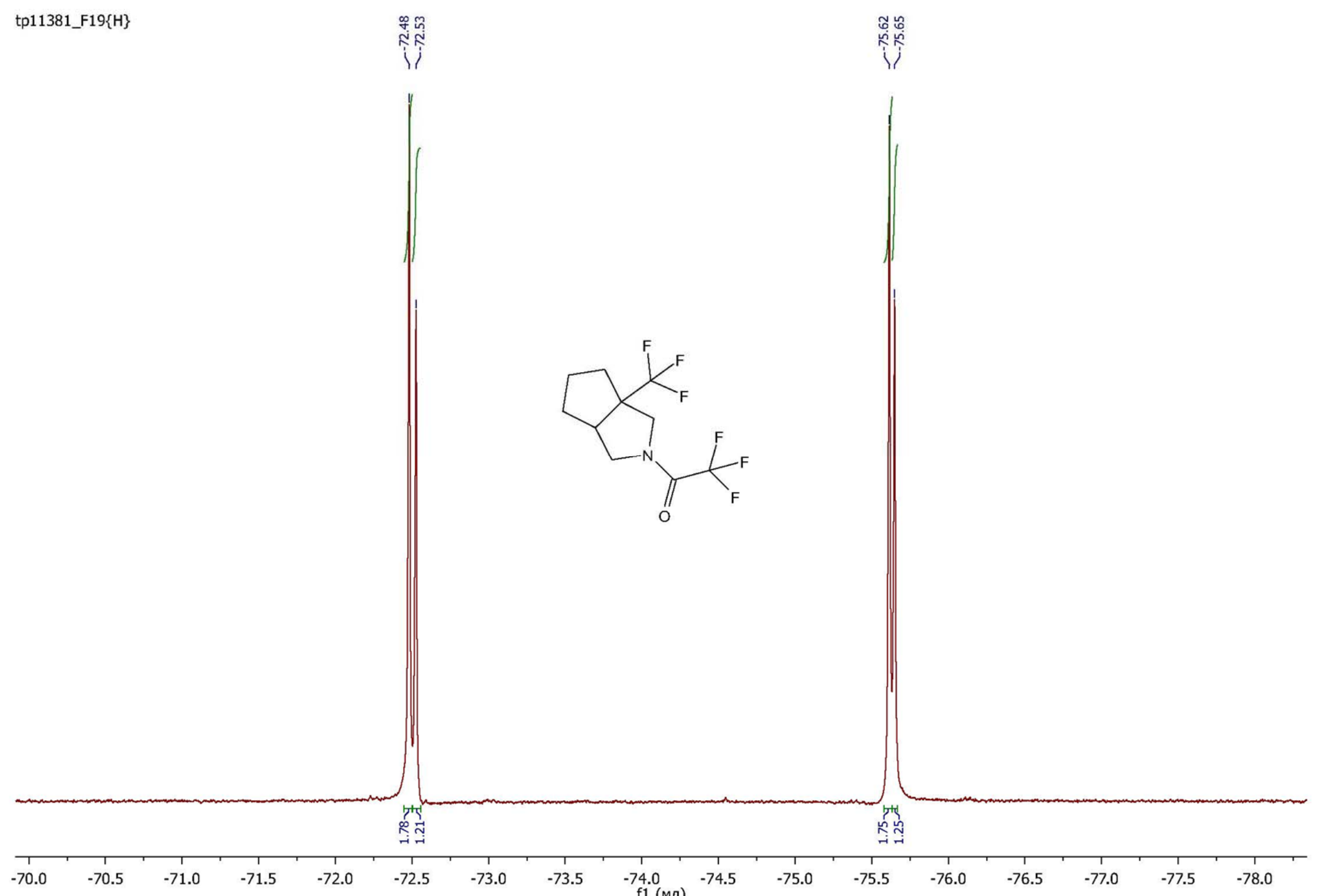




\section{Compound 41a}

R1306844

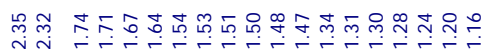

$\mathrm{X}_{\mathrm{CF}_{3}}^{\mathrm{CO}_{2} \mathrm{Et}}$
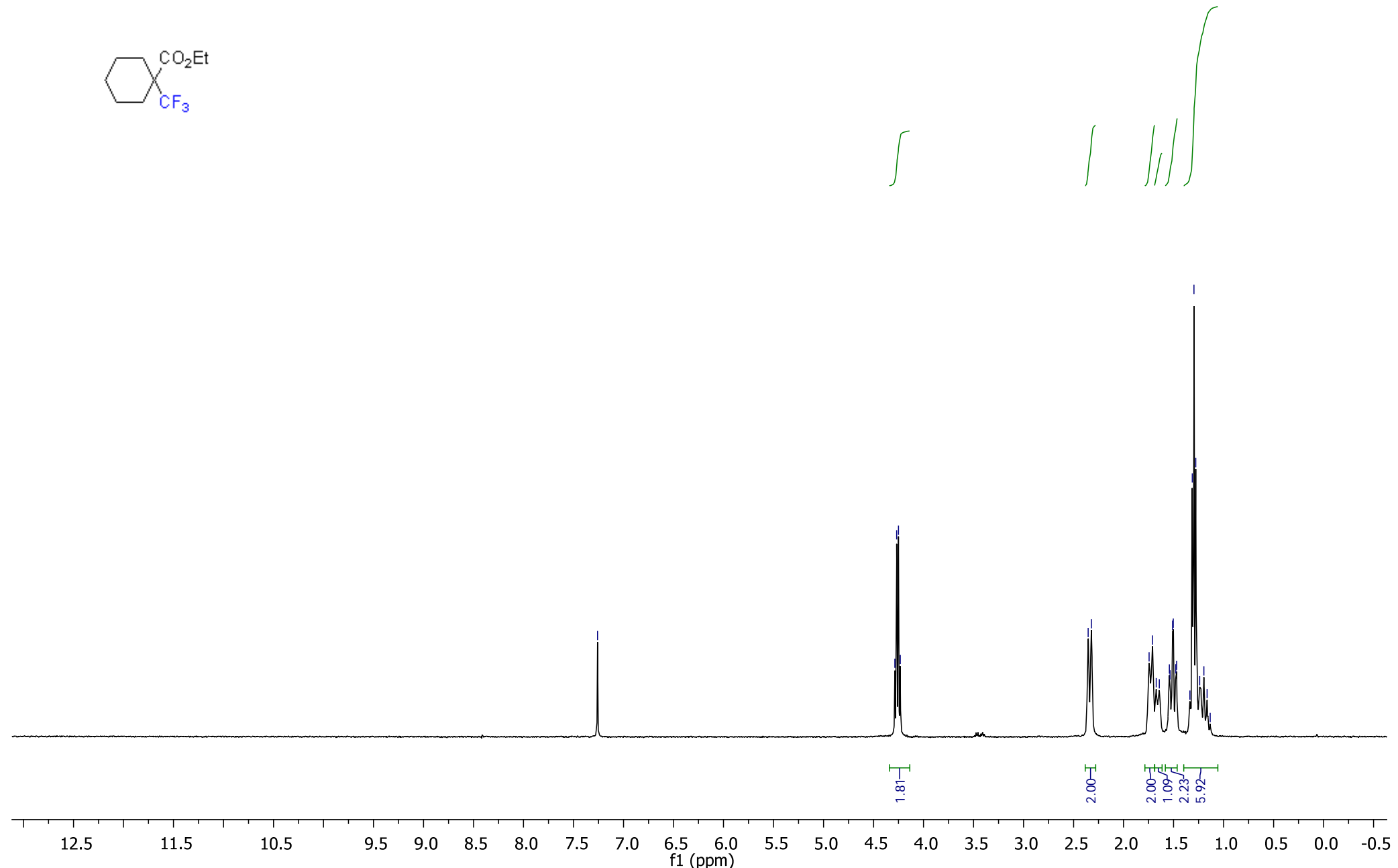
R1306844_C13

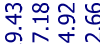

1111
굿음

is

\&

फे กํูกูก

I

กิ่

।र<smiles>CCOC1([13CH3])CCCCC1</smiles>

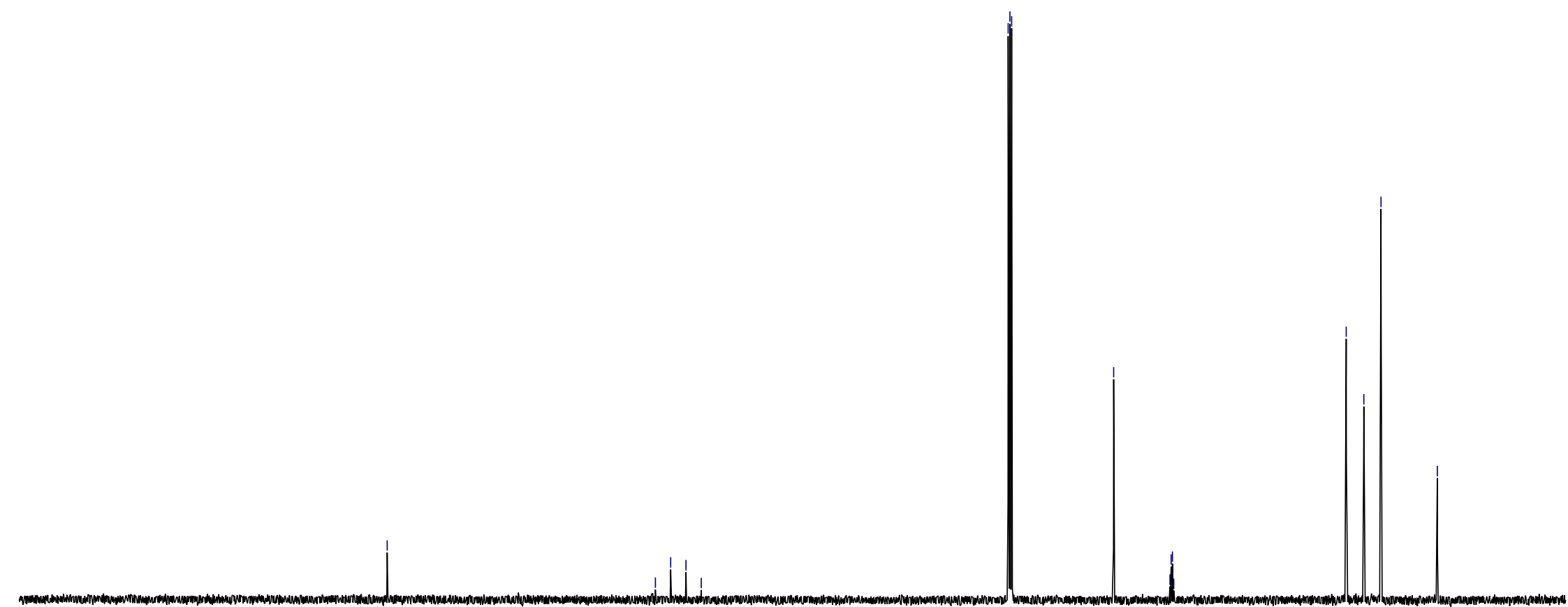


R1306844_F19 $\{1 \mathrm{H}\}$
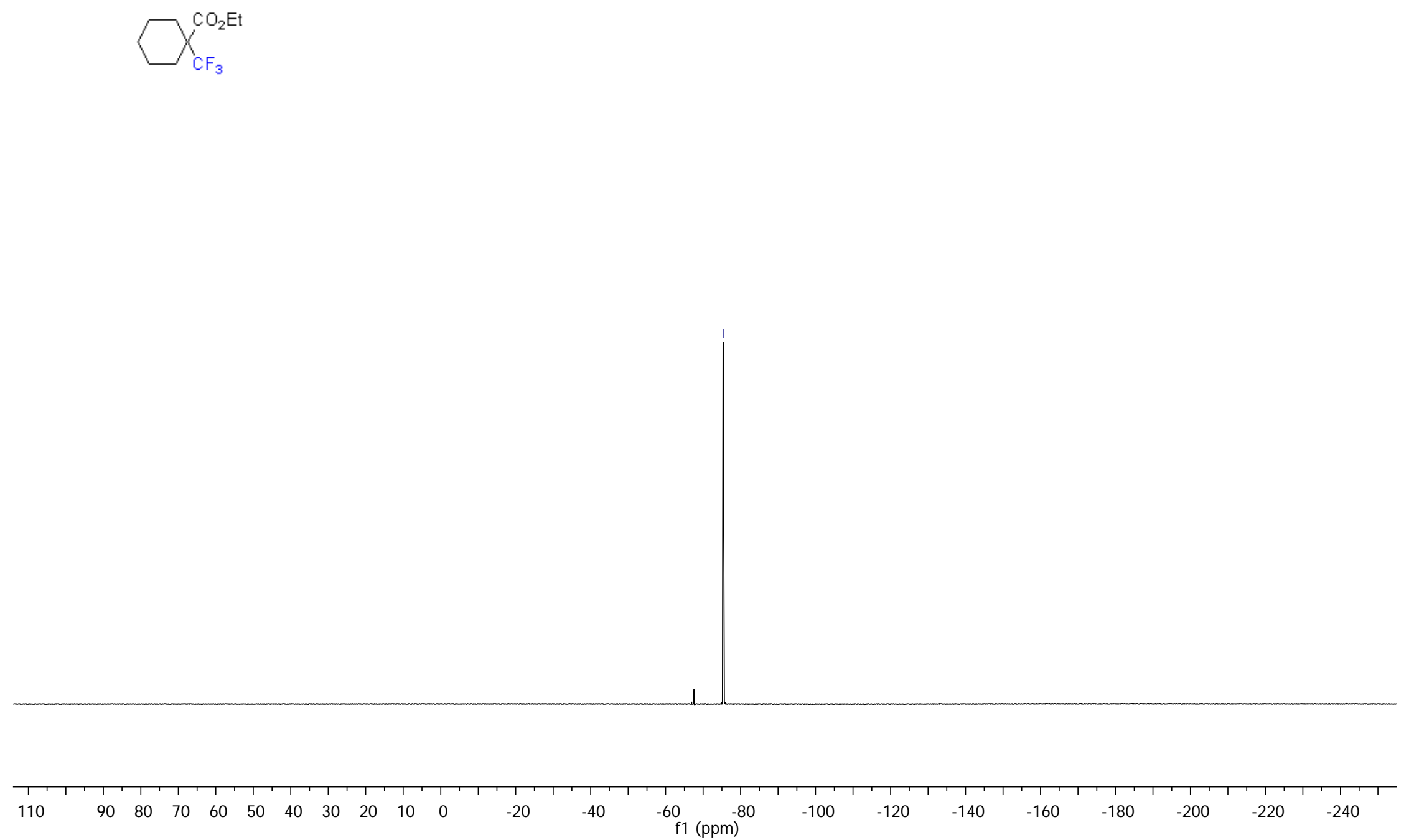


\section{Compound 42a}

igm11740<smiles>COC(=O)C12CCC(C(F)(F)F)(CC1)CC2</smiles>

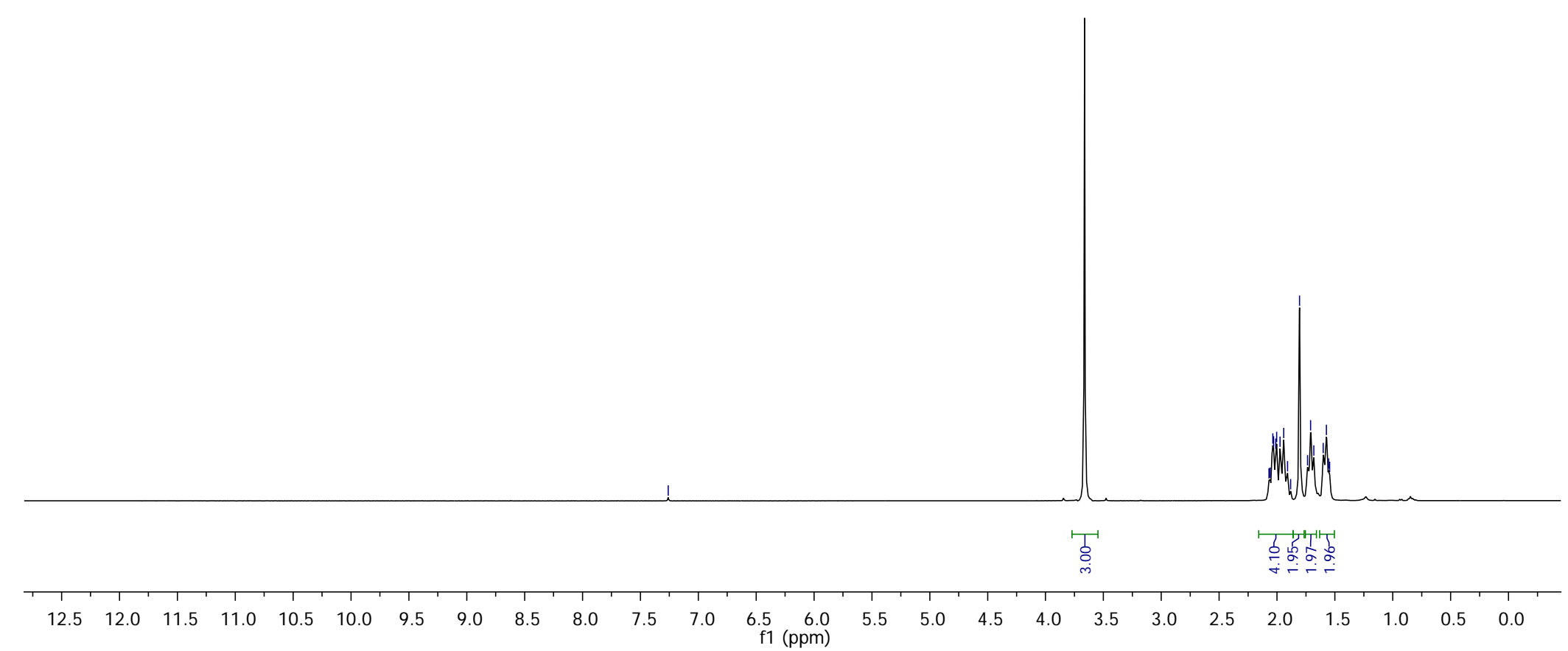


igm11740_C13

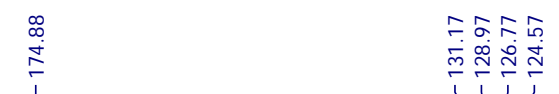

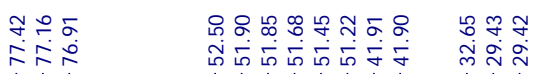

1111

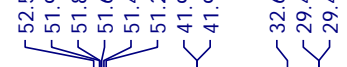

$\mathrm{MeO}_{2} \mathrm{C} \longrightarrow \mathrm{CF}_{3}$
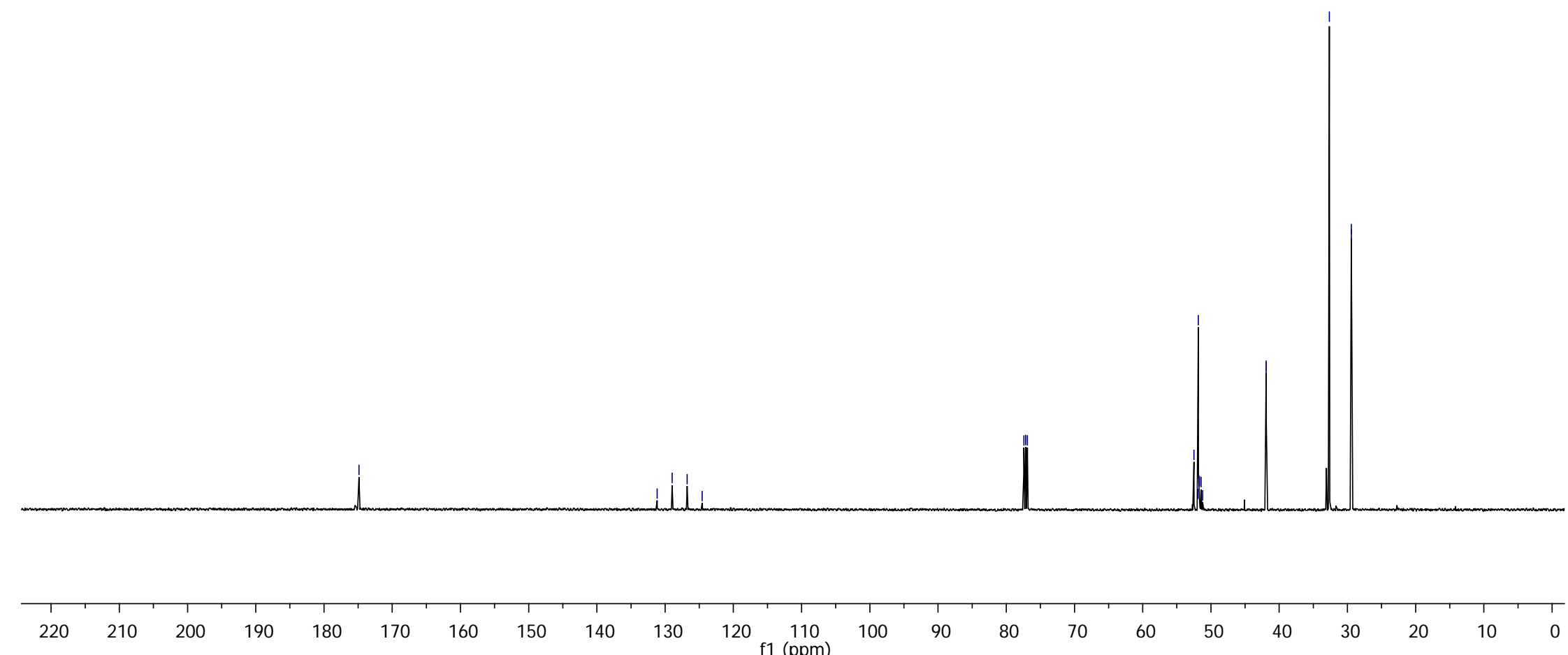
igm11740_F19 $\{\mathrm{H}\}$

$\mathrm{MeO}_{2} \mathrm{C} \longrightarrow \mathrm{CF}_{3}$

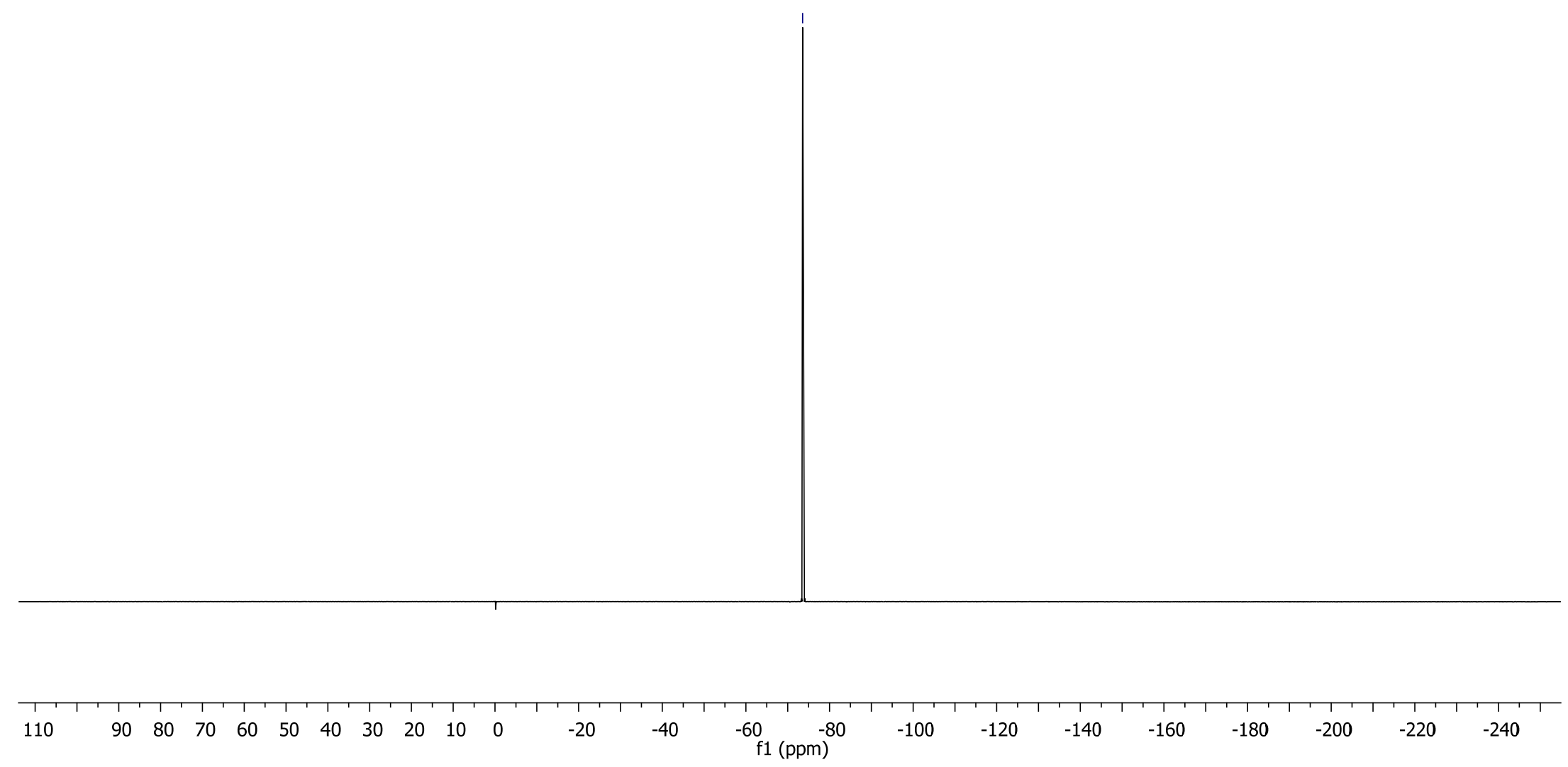




\section{Compound 43a}

R1240029

$\mathrm{HO}_{2} \mathrm{C} \longrightarrow \mathrm{CF}_{3}$

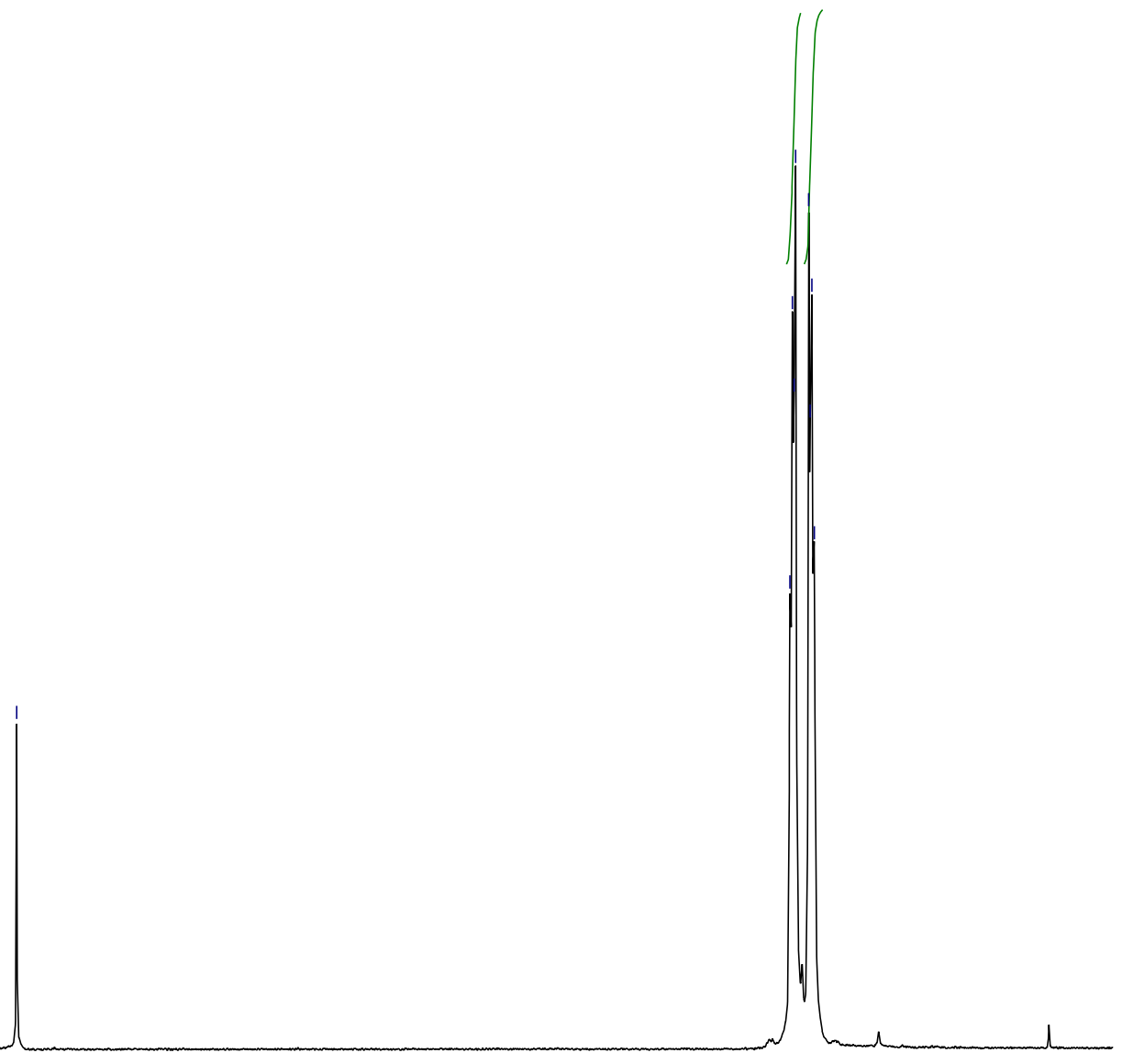


R1240029_C13<smiles>O=C(O)C1CCC(C(F)(F)F)CC1</smiles> 
R1240029_F19 $\{\mathrm{H}\}$

$19 F-\{1 \mathrm{H}\}$

$\mathrm{HO}_{2} \mathrm{C}-\mathrm{CF}_{3}$

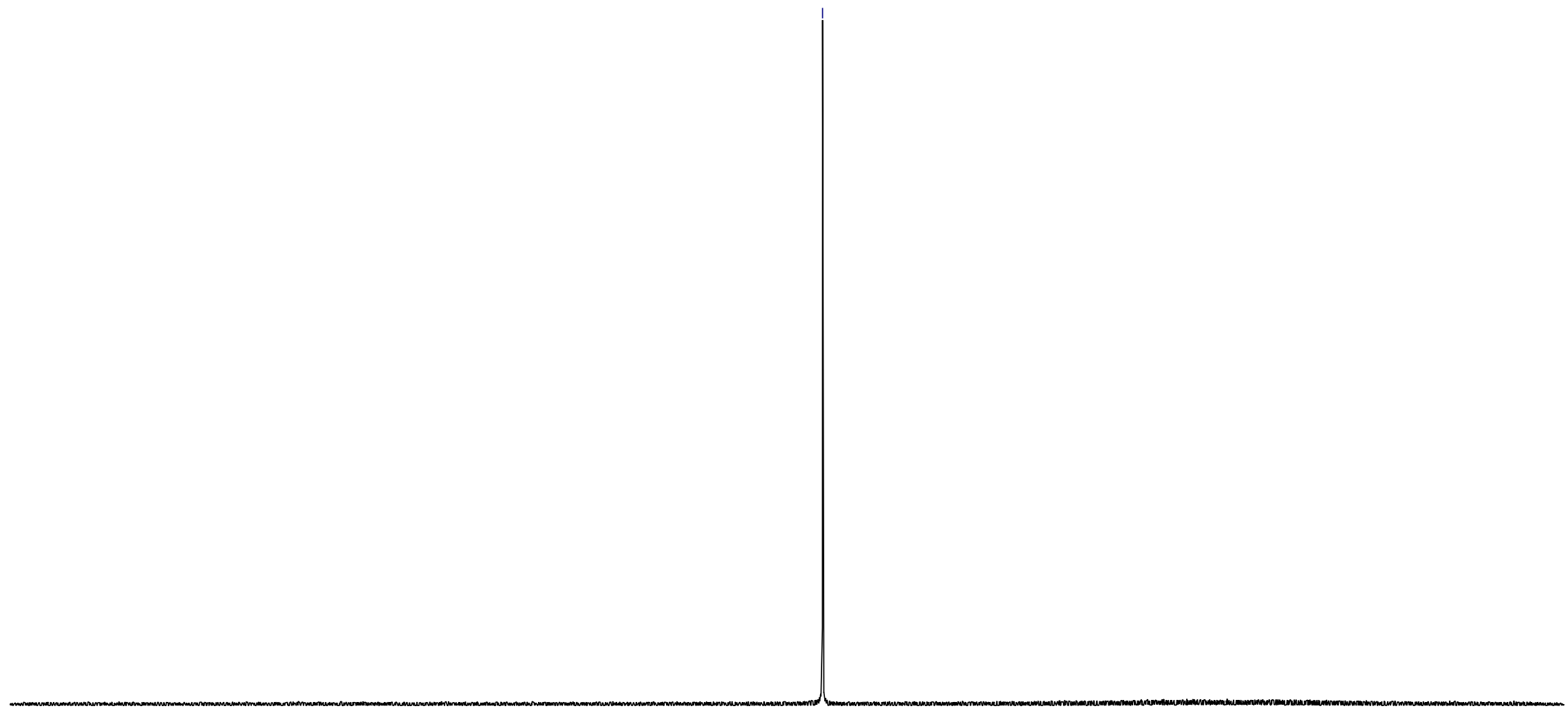




\section{Compound 2b}

R1278541

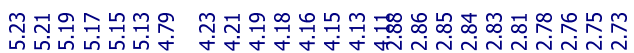

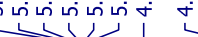

$\overbrace{\mathrm{H}}^{{ }_{\mathrm{N}}^{\mathrm{HCl}}}-\mathrm{CF}_{3}$
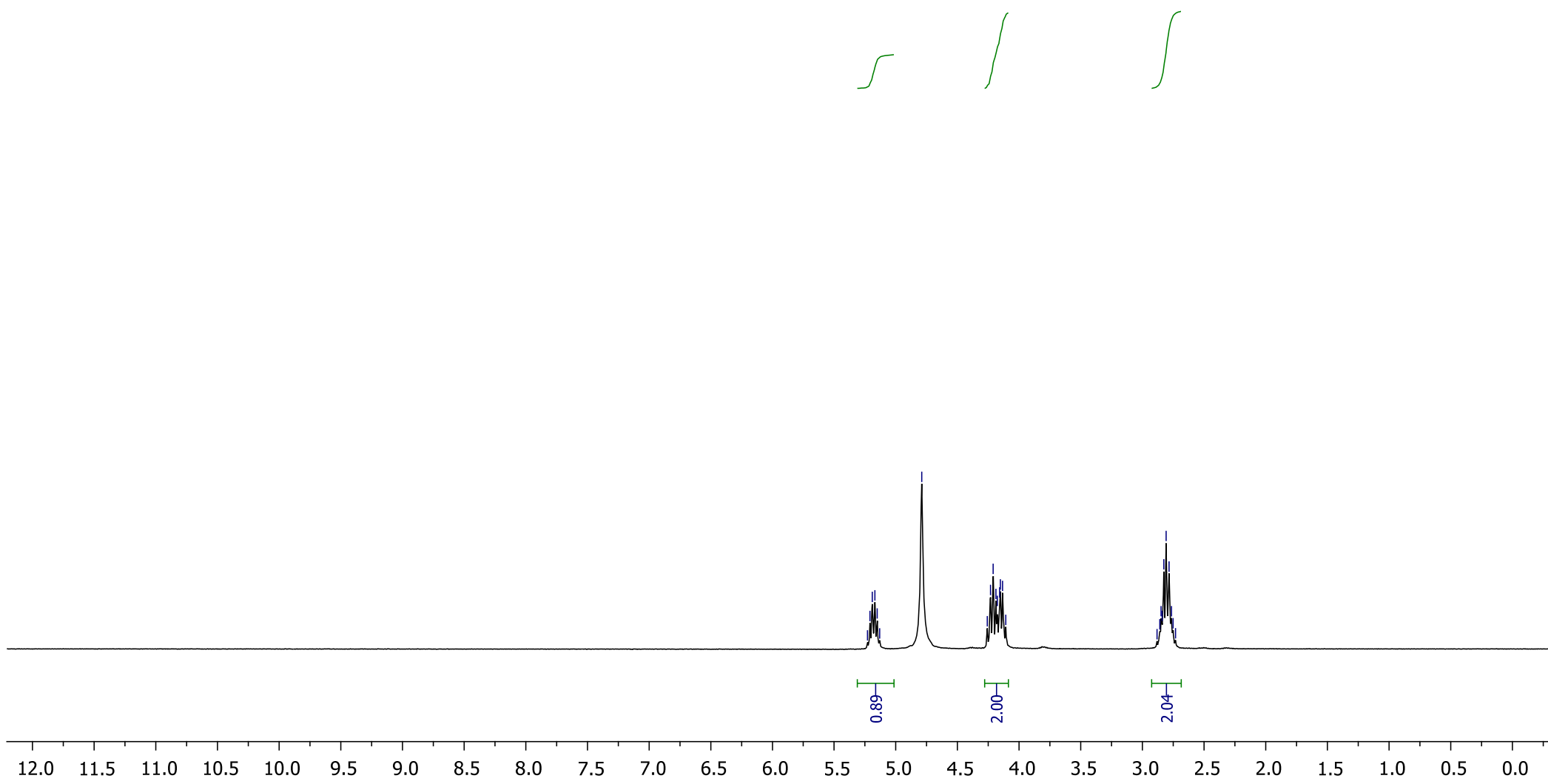
R1278541_C13

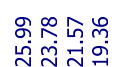

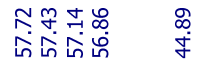

$\stackrel{8}{\stackrel{8}{\infty}}$

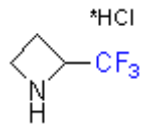

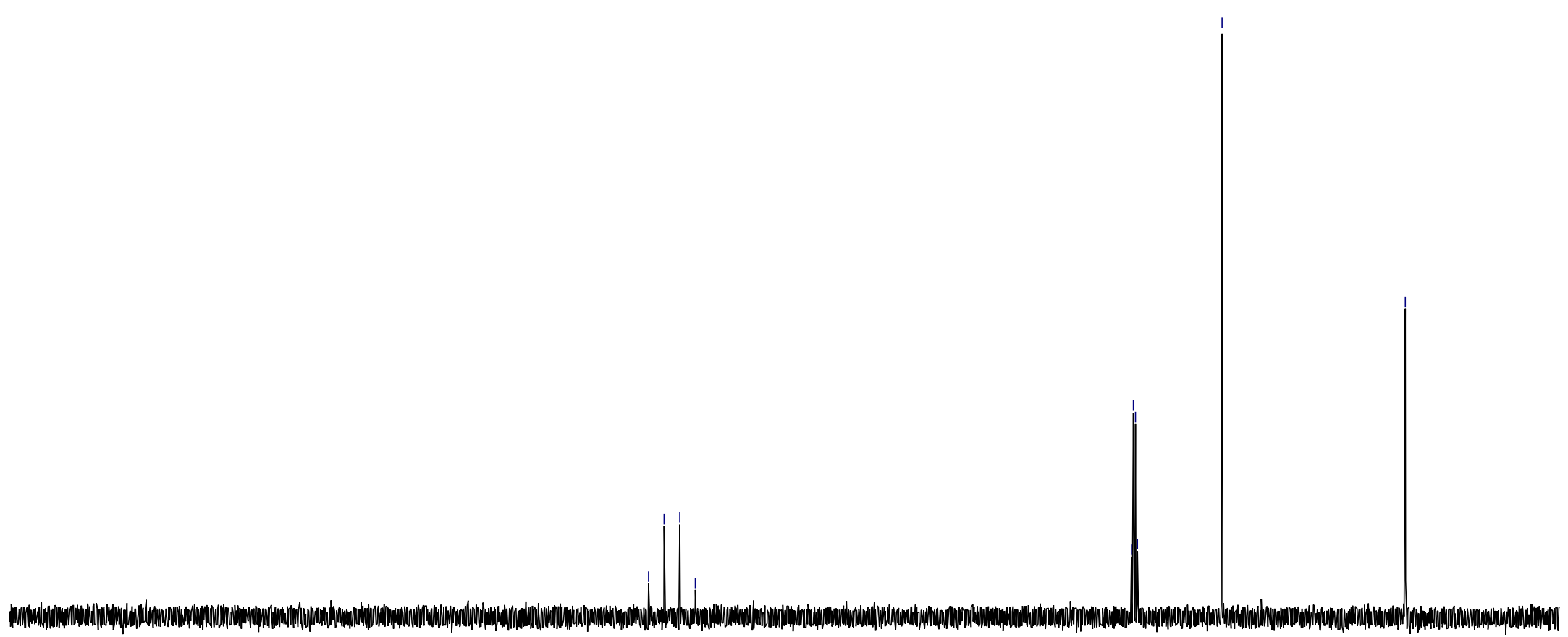

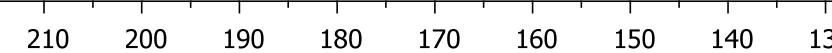

$120-110+100$

90

$80 \quad 70$

60

$50 \quad 40 \quad 30$

20 
R1278541_F19\{H\}

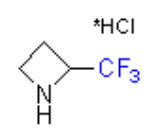

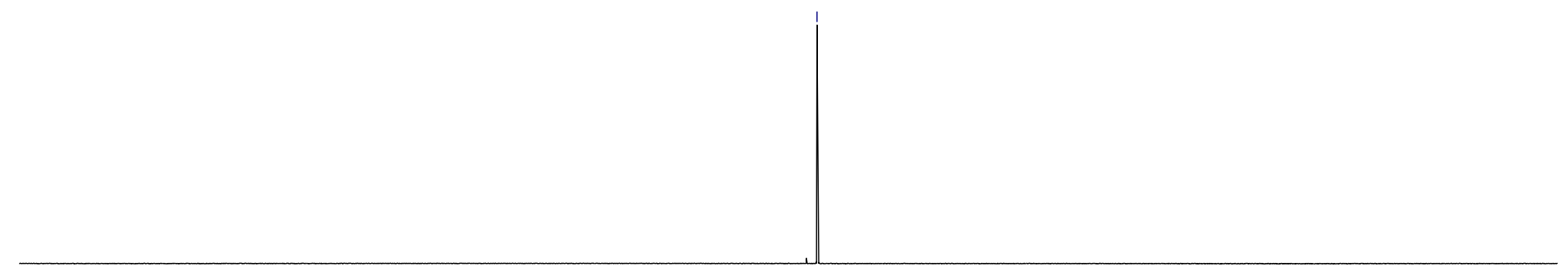

$110 \quad \begin{array}{lllllllllllllllllllllllllll} & 90 & 70 & 60 & 50 & 40 & 30 & 20 & 10 & 0 & -20 & -40 & -60 & -80 & -100 & -120 & -140 & -160 & -180 & -200 & -220 & -240\end{array}$ 


\section{Compound 6b}

R94925

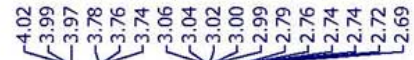

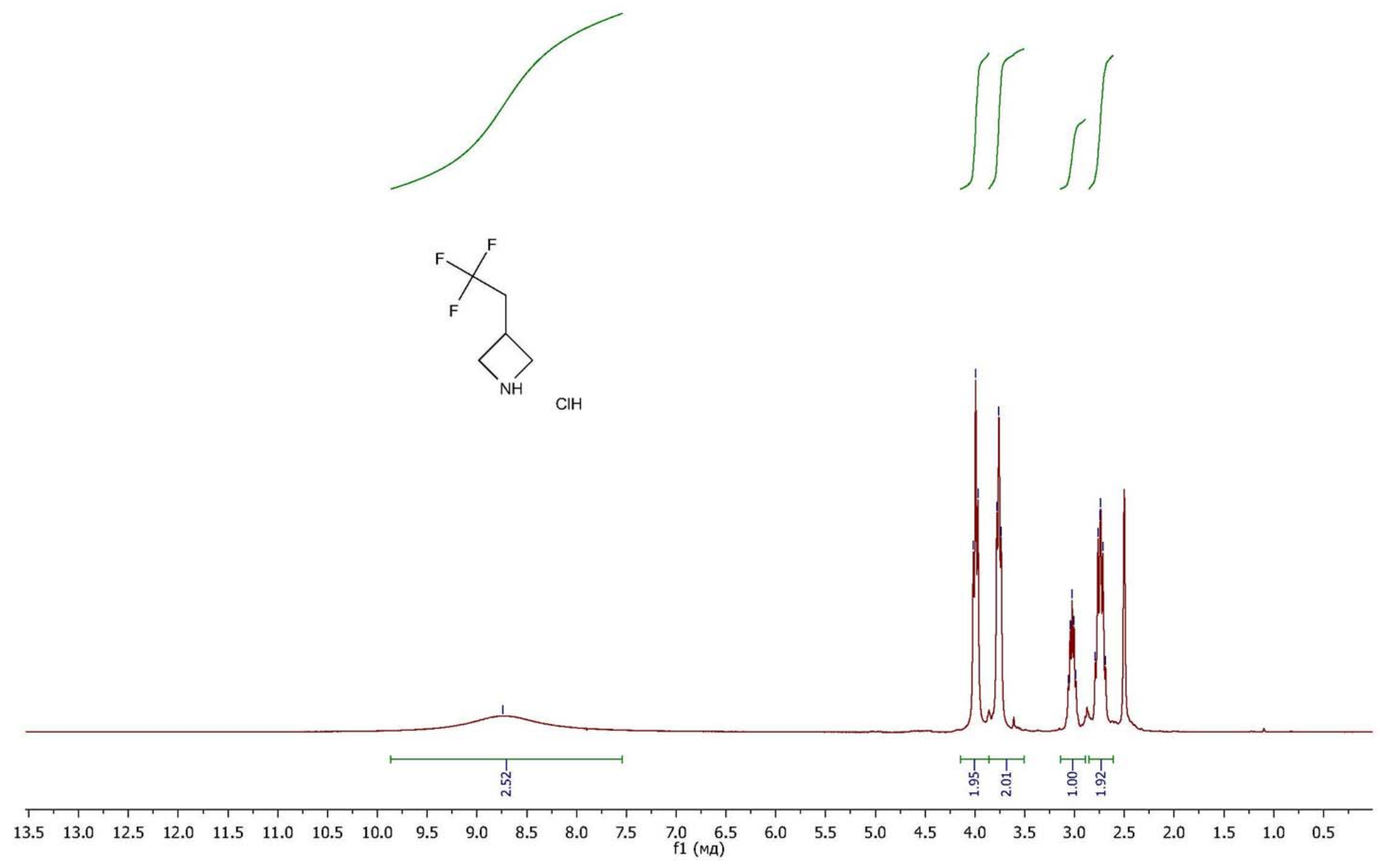



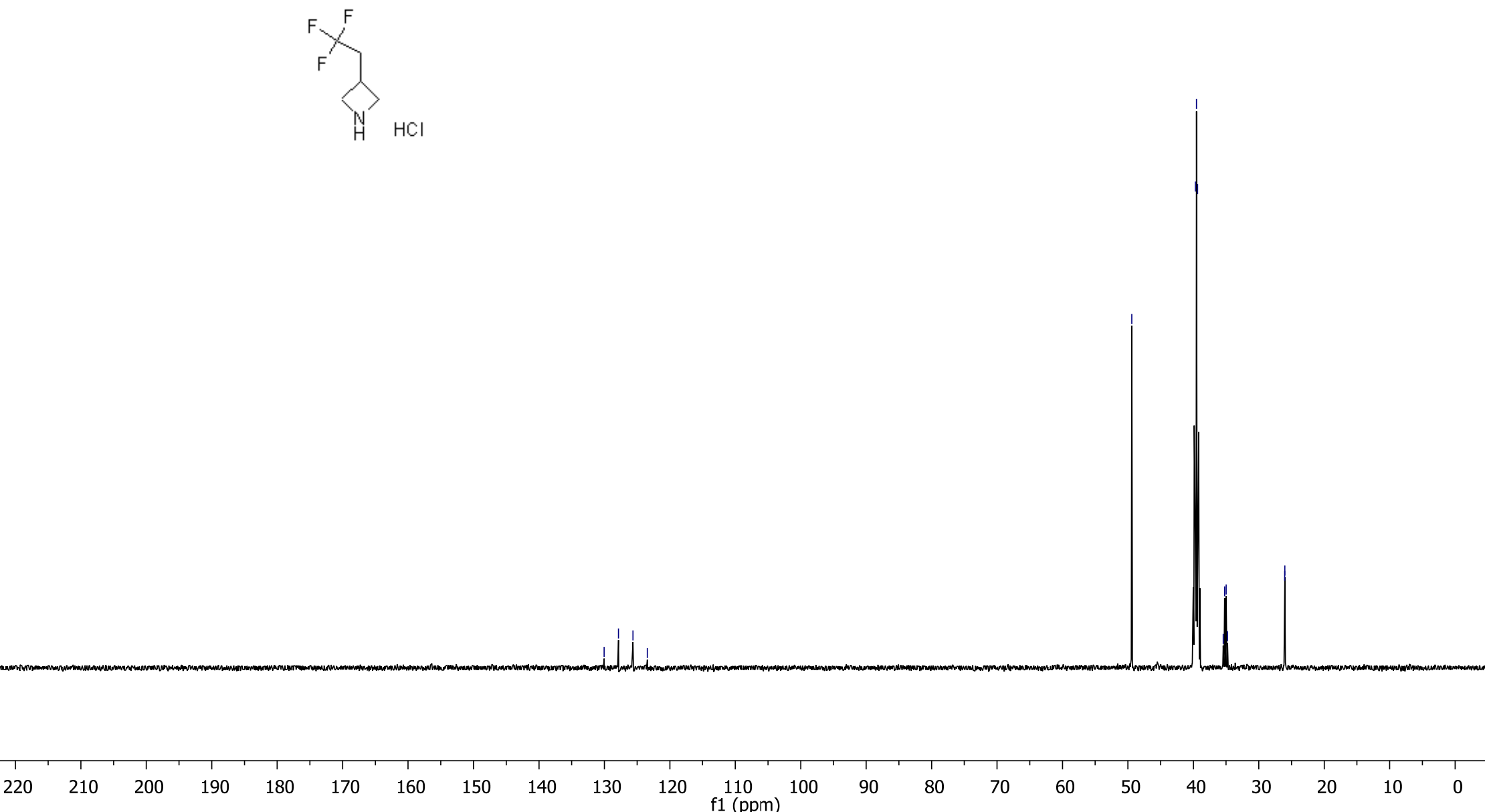
R94925_F19(H1)

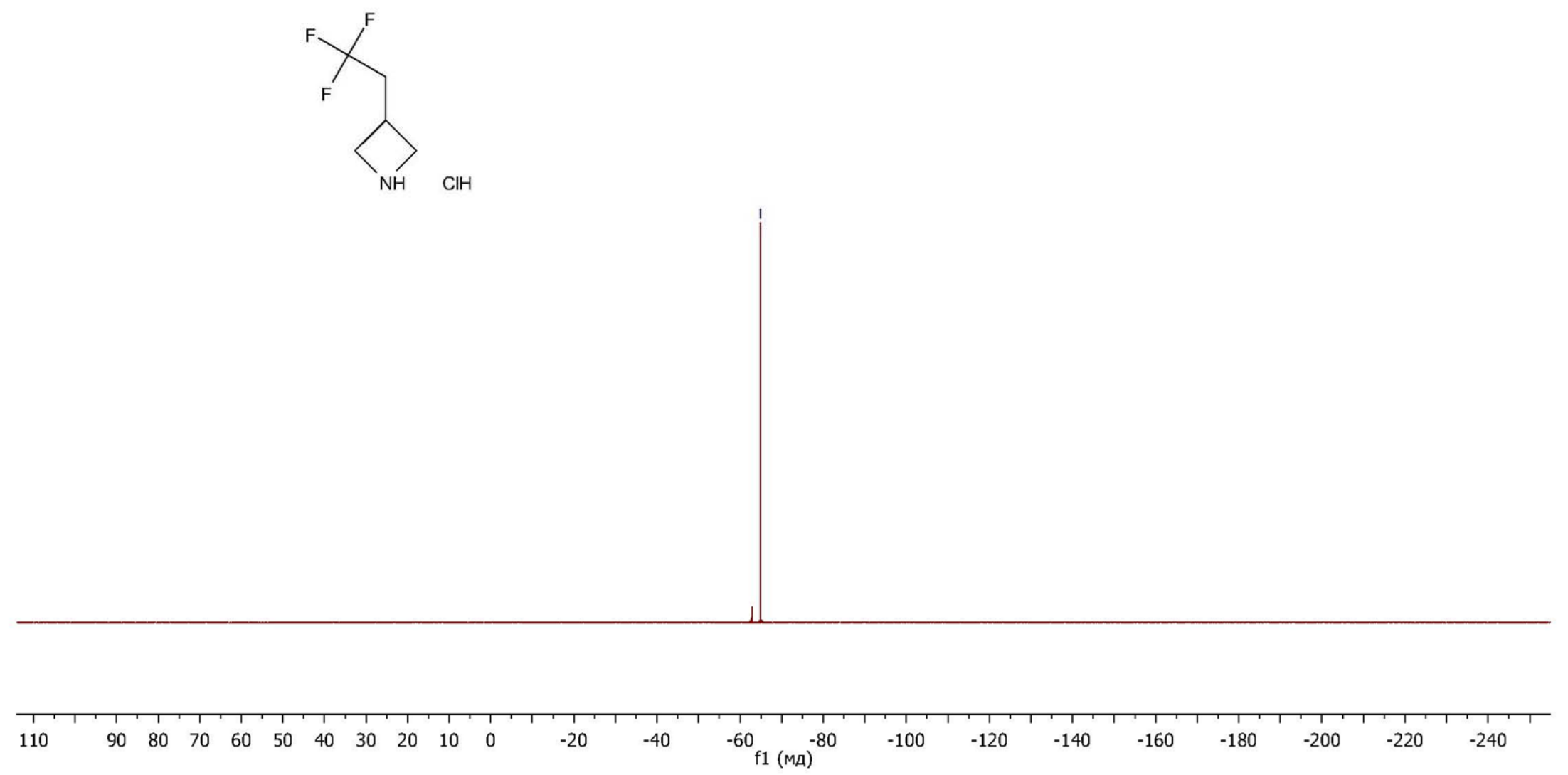




\section{Compound 7b}

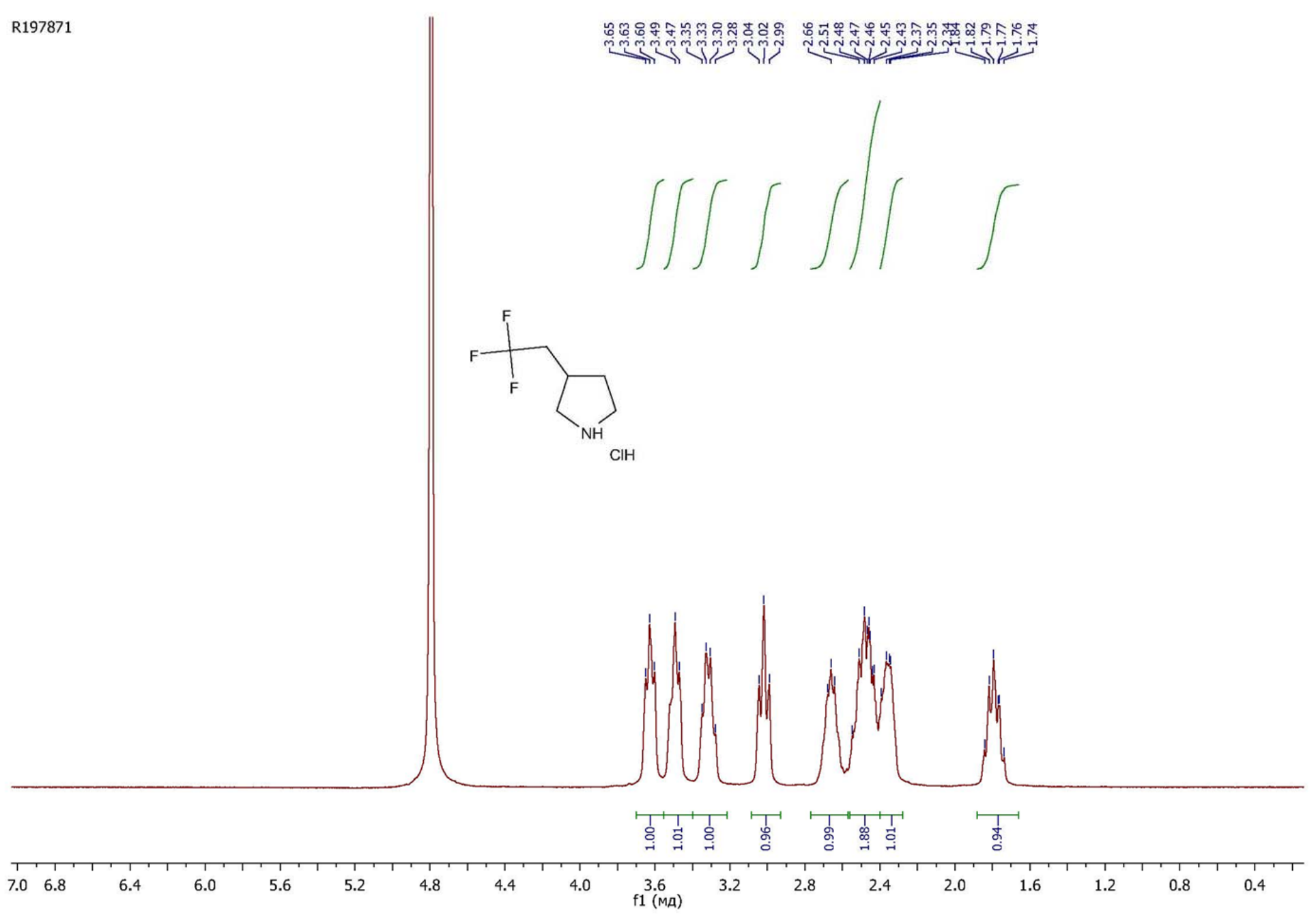




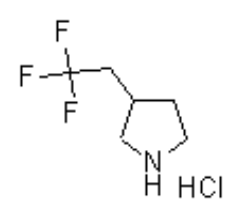

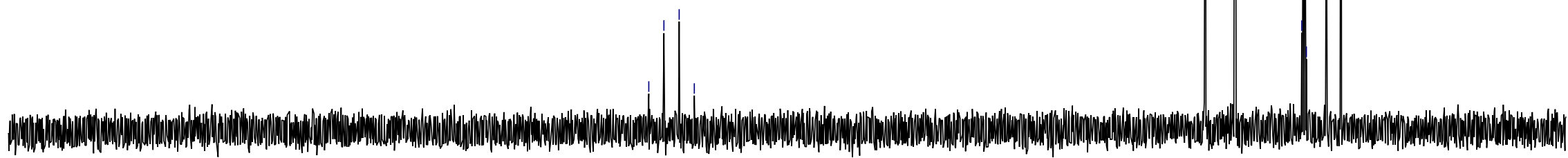


R197871_F19(H1)

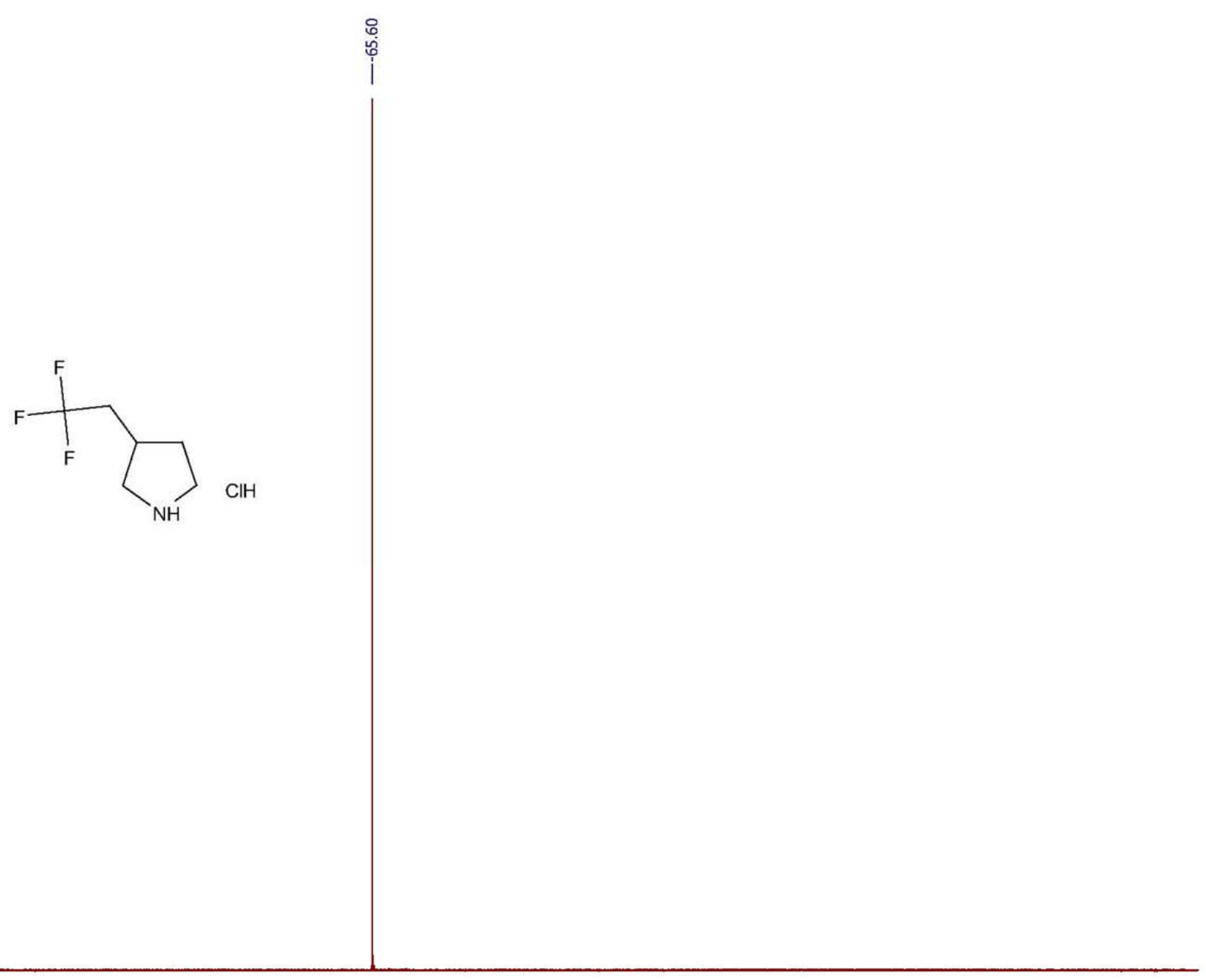

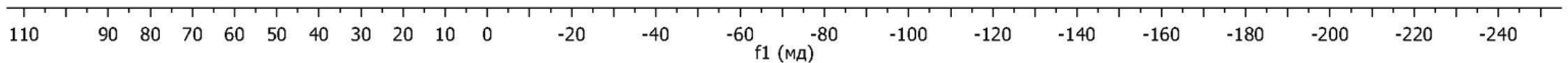




\section{Compound 8b}

R593113

लं

$\iint$
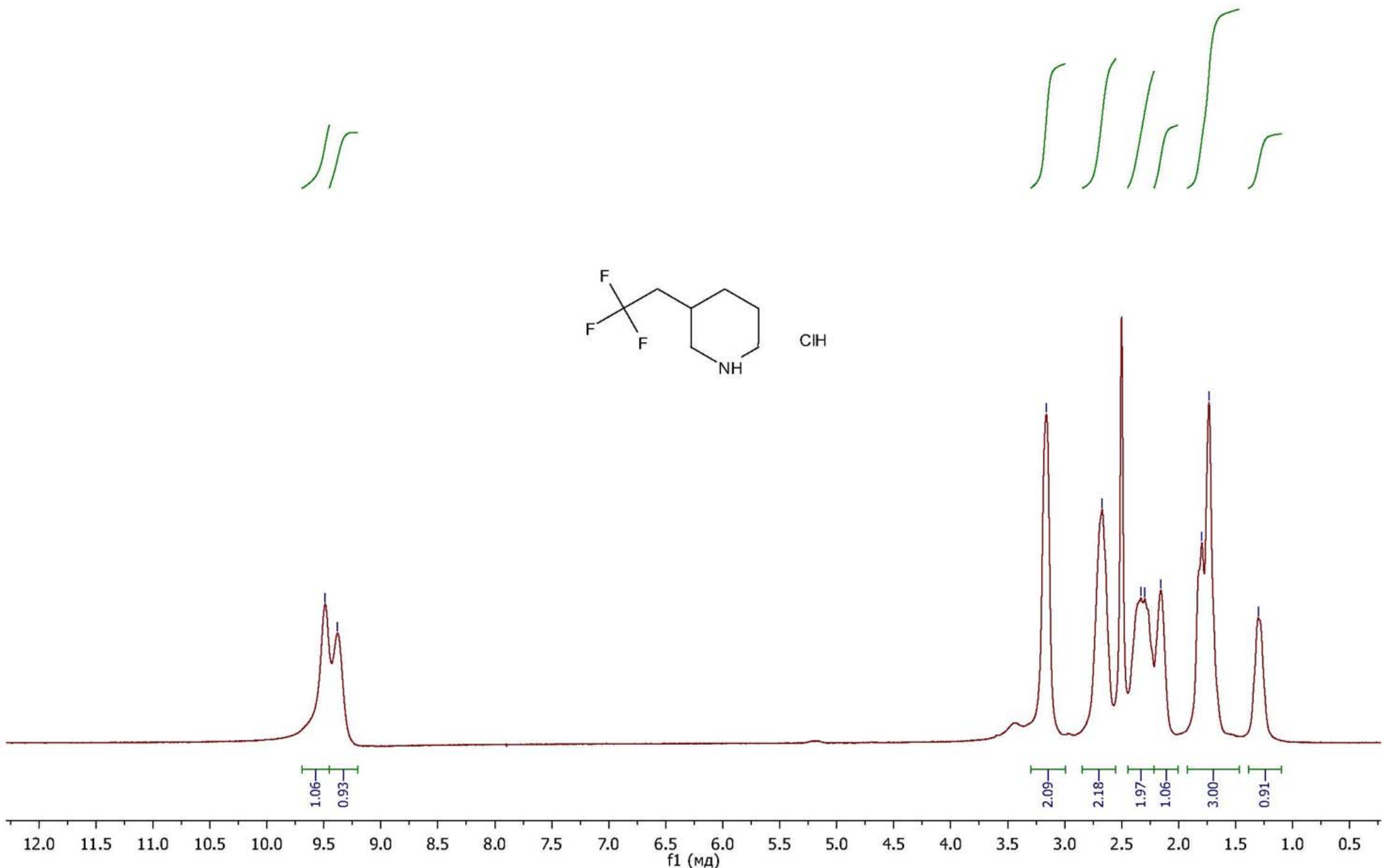
R593113_C13

유ㅇㅝㅜ응

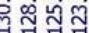

तार

$\mathrm{C}_{\mathrm{NH}}^{\mathrm{C}}$

$\mathrm{CIH}$
กิ

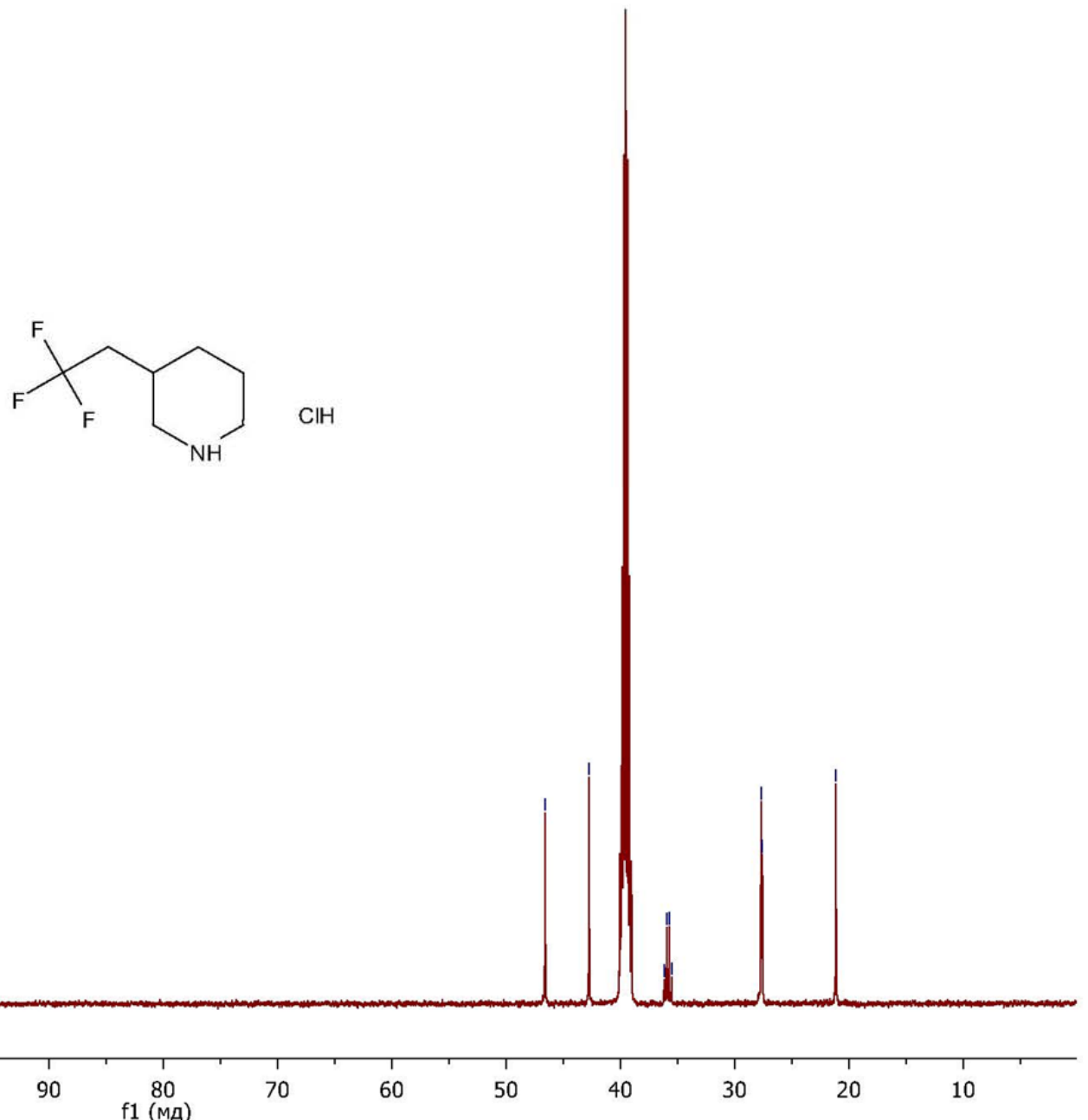


R593113_F19

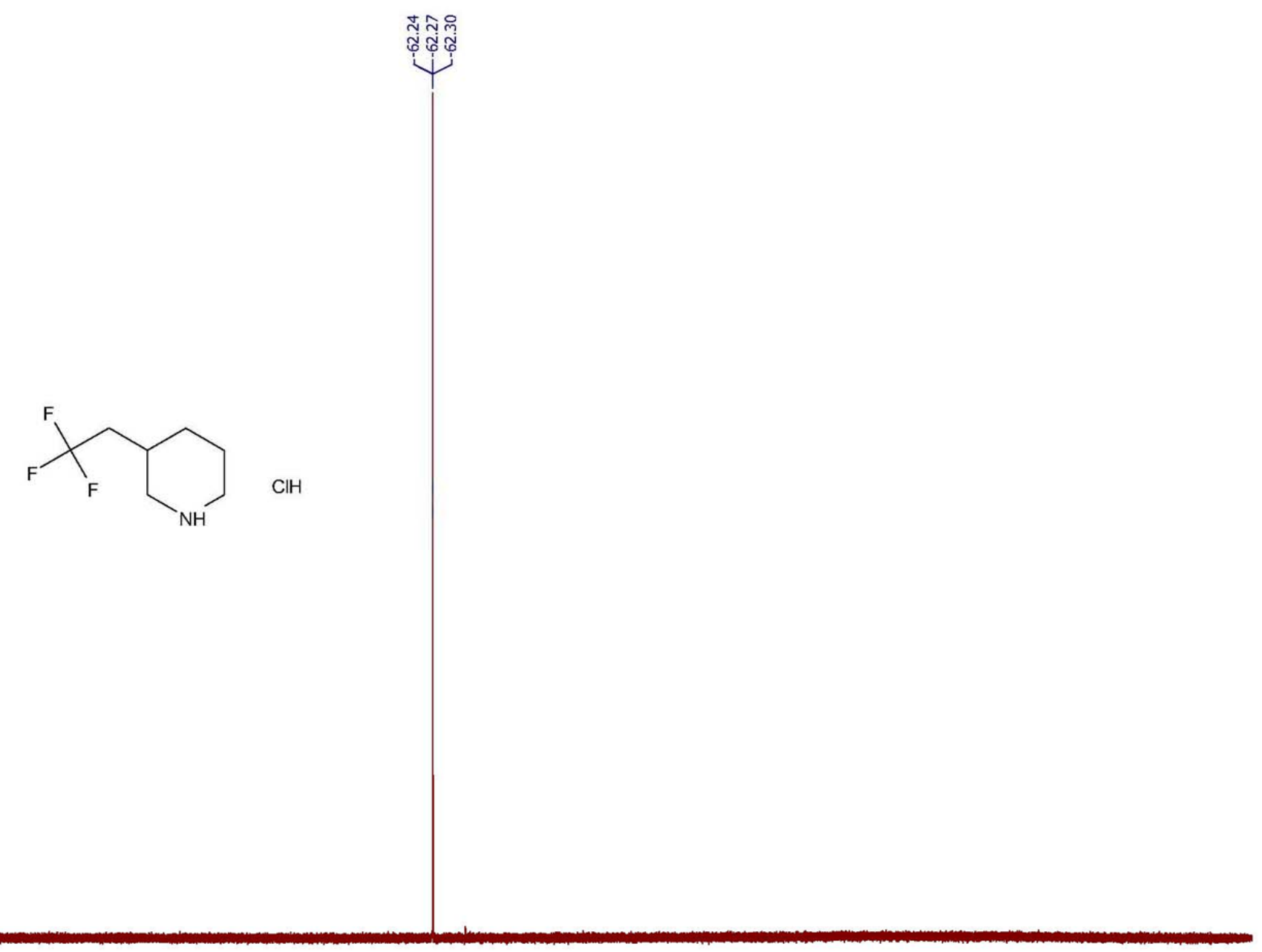

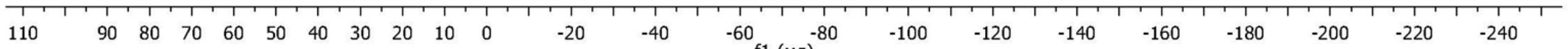




\section{Compound 9b}

R811702

के
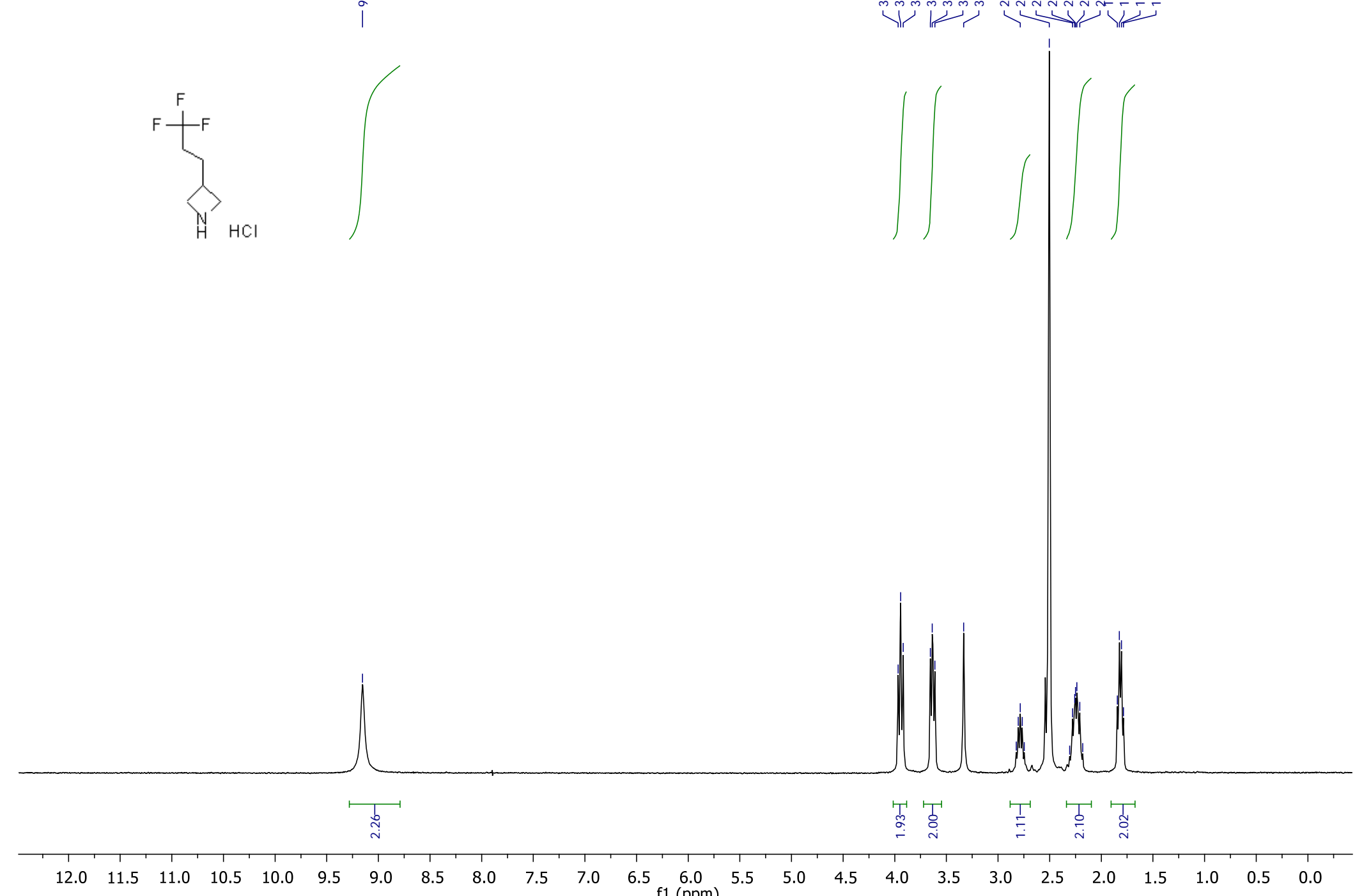
R811702_C13

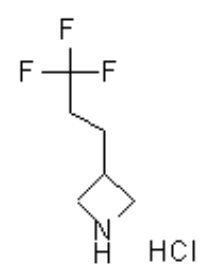

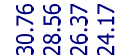

1111

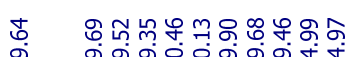

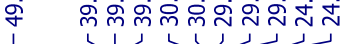

舟

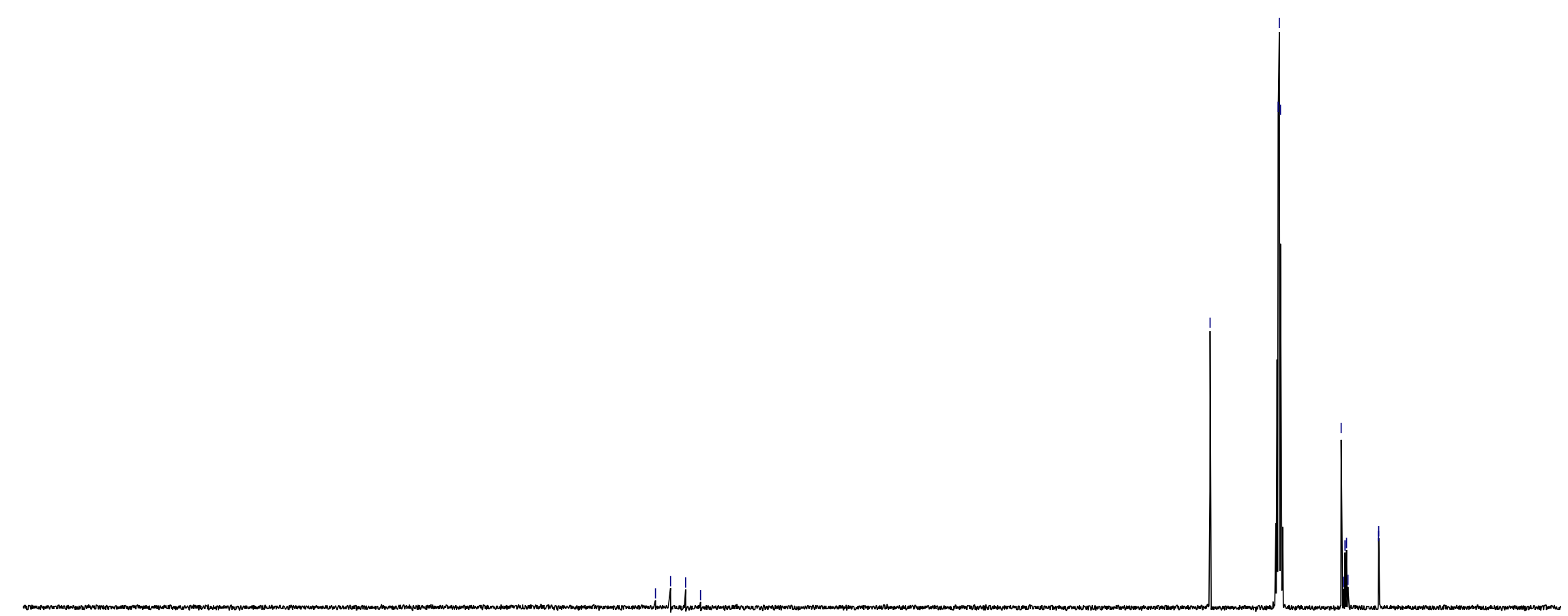

$\begin{array}{lllll} & & & & \\ 220 & 210 & 200 & 190 & 180\end{array}$

$180 \quad 170$

$160 \quad 150$

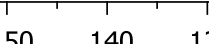

120

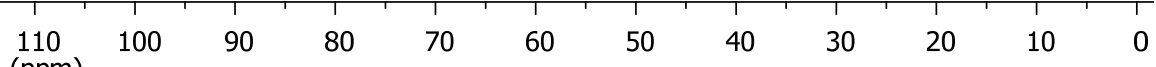


$\mathrm{R} 811702 \mathrm{~F} 19\{\mathrm{H}\}$ $19 F-\{1 \mathrm{H}\}$

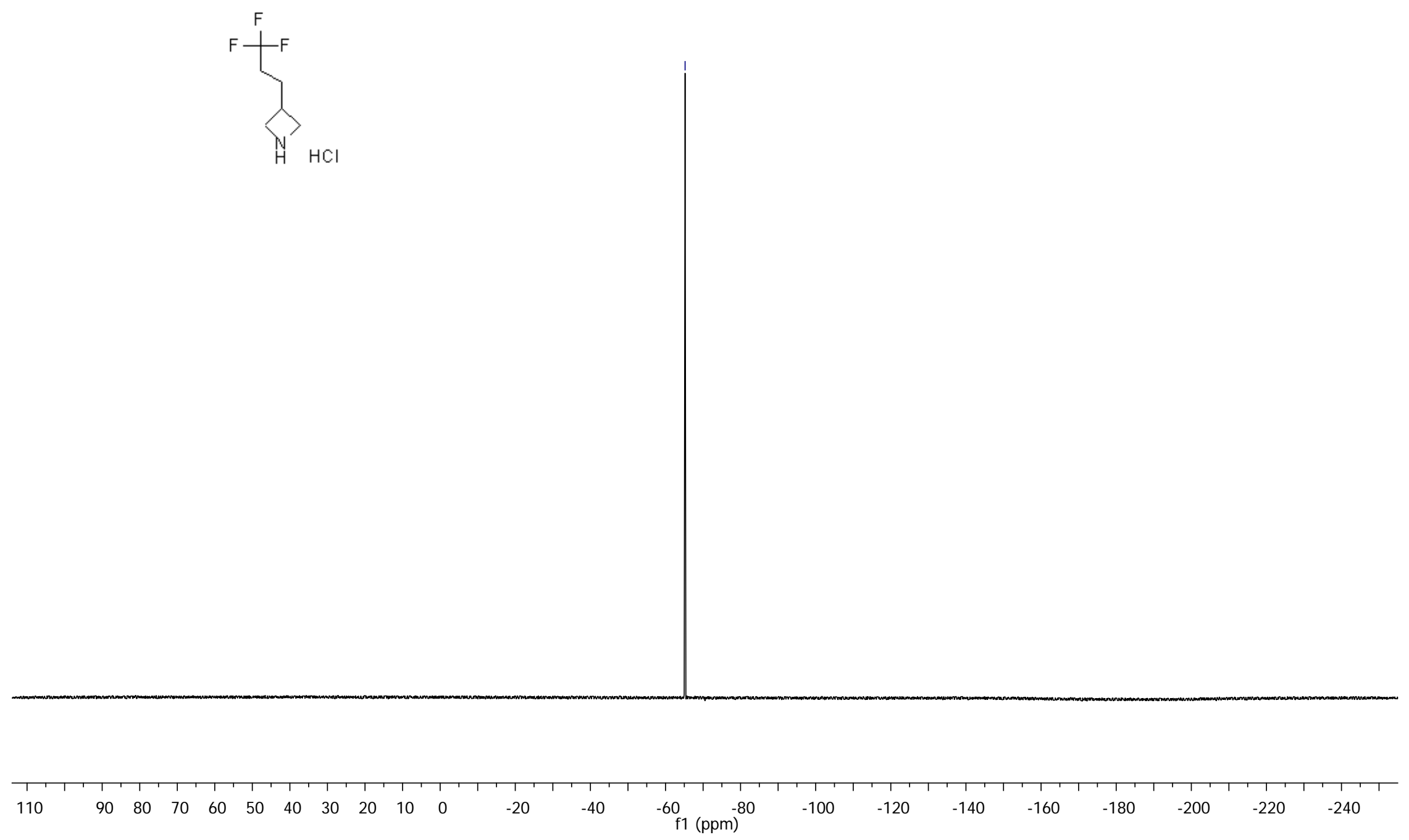




\section{Compound 10b}

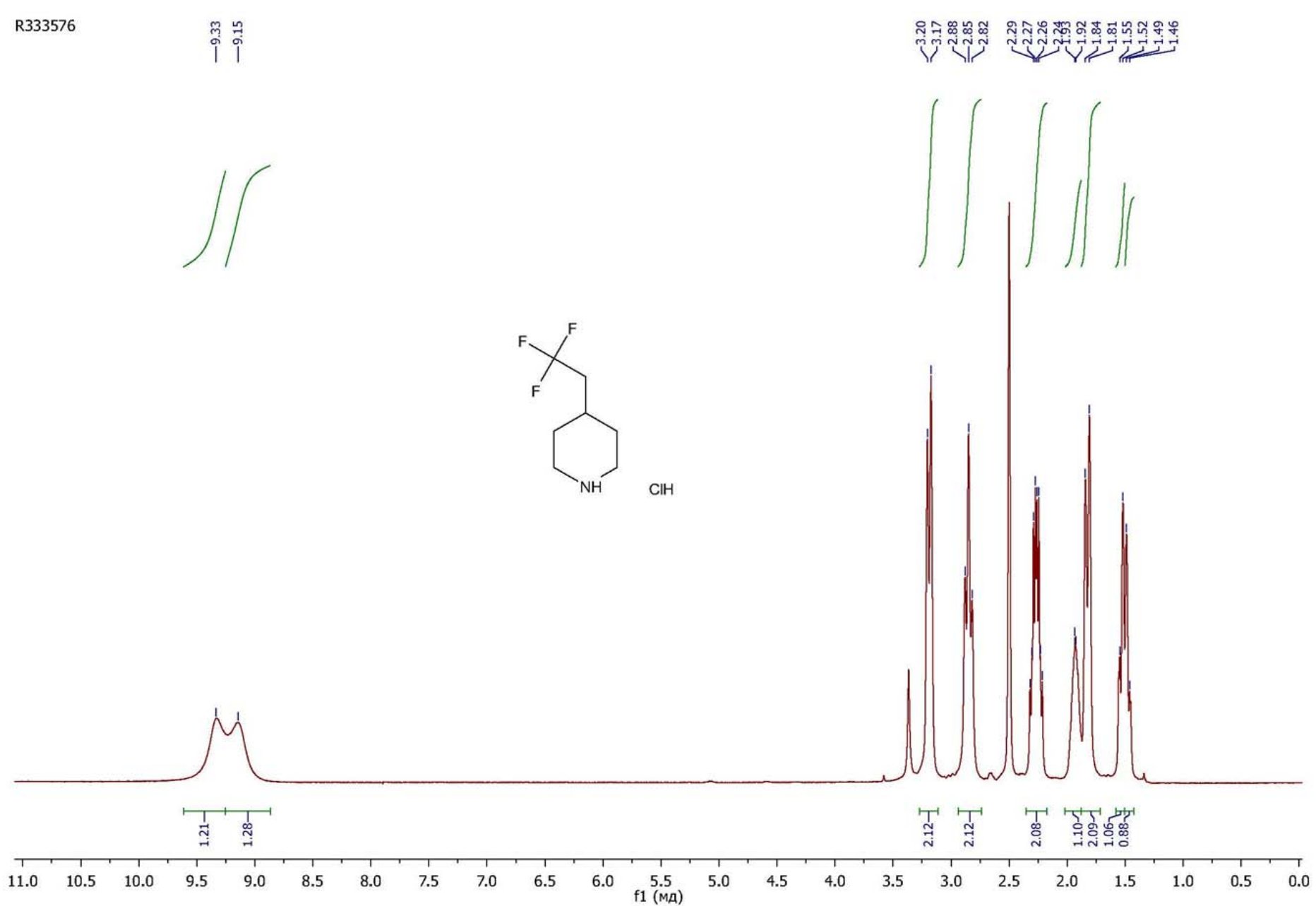


R333576_C13

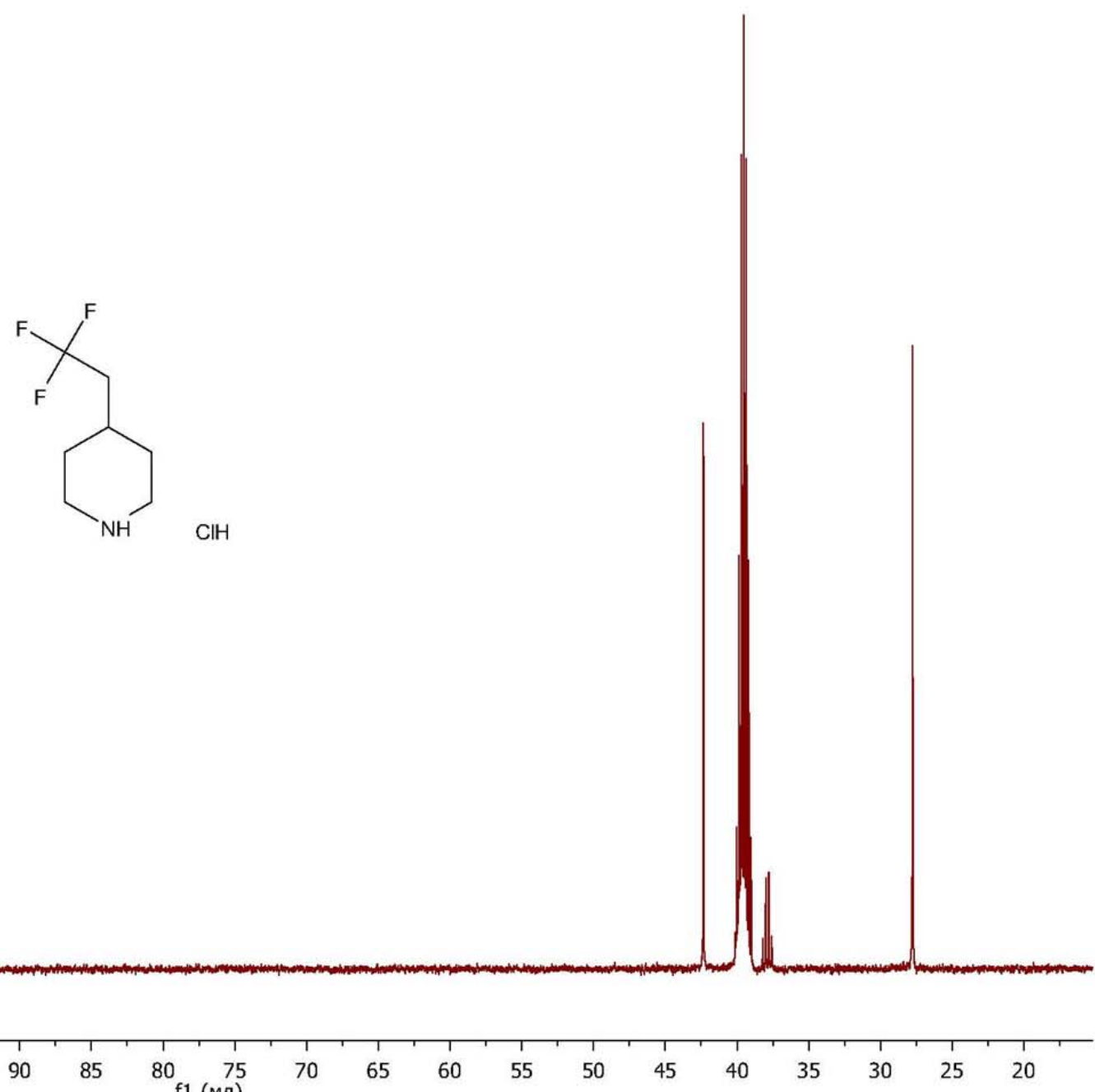


R333576_F19\{H\}
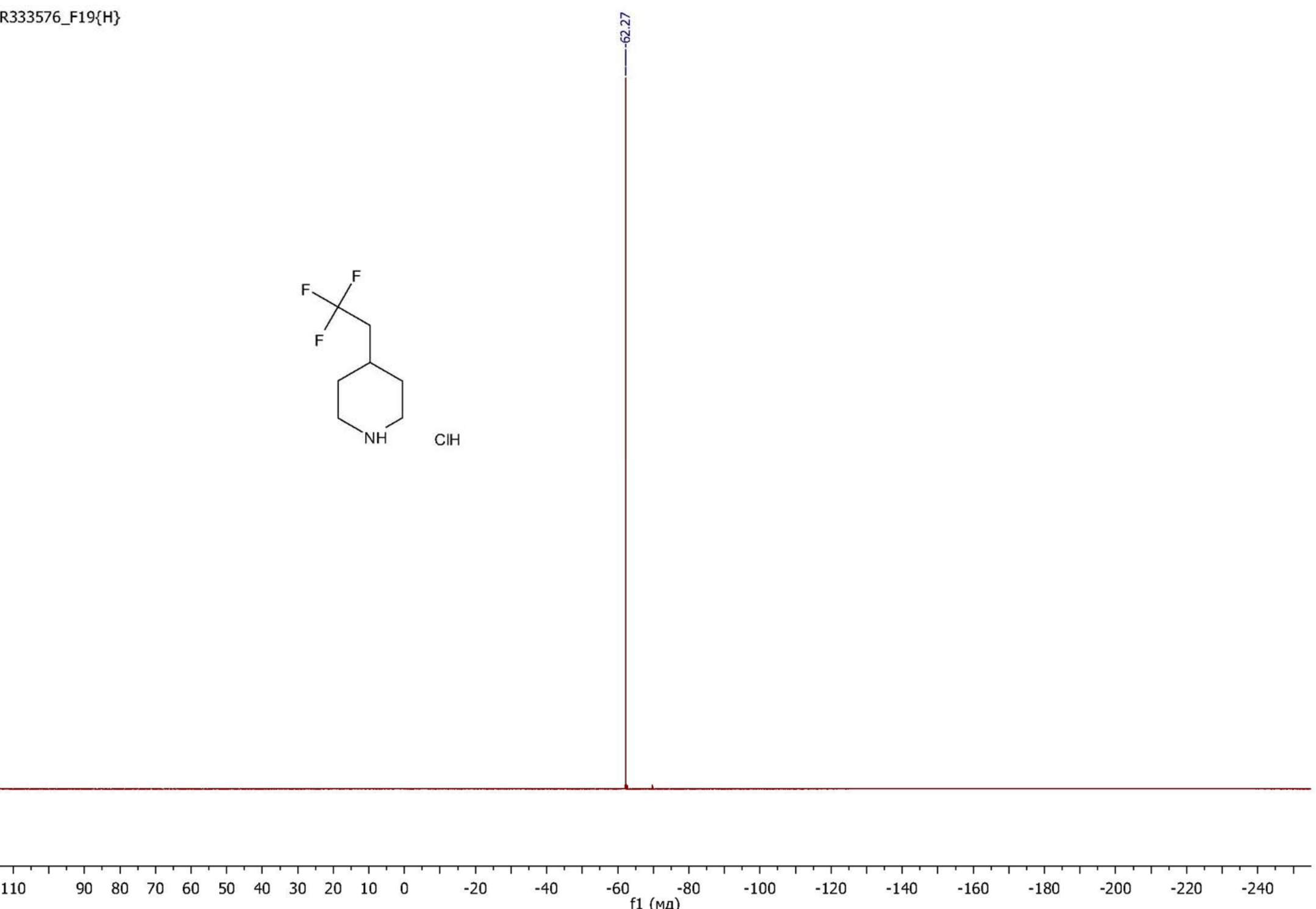


\section{Compound 11b}

R1066471

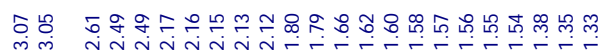

Y Y
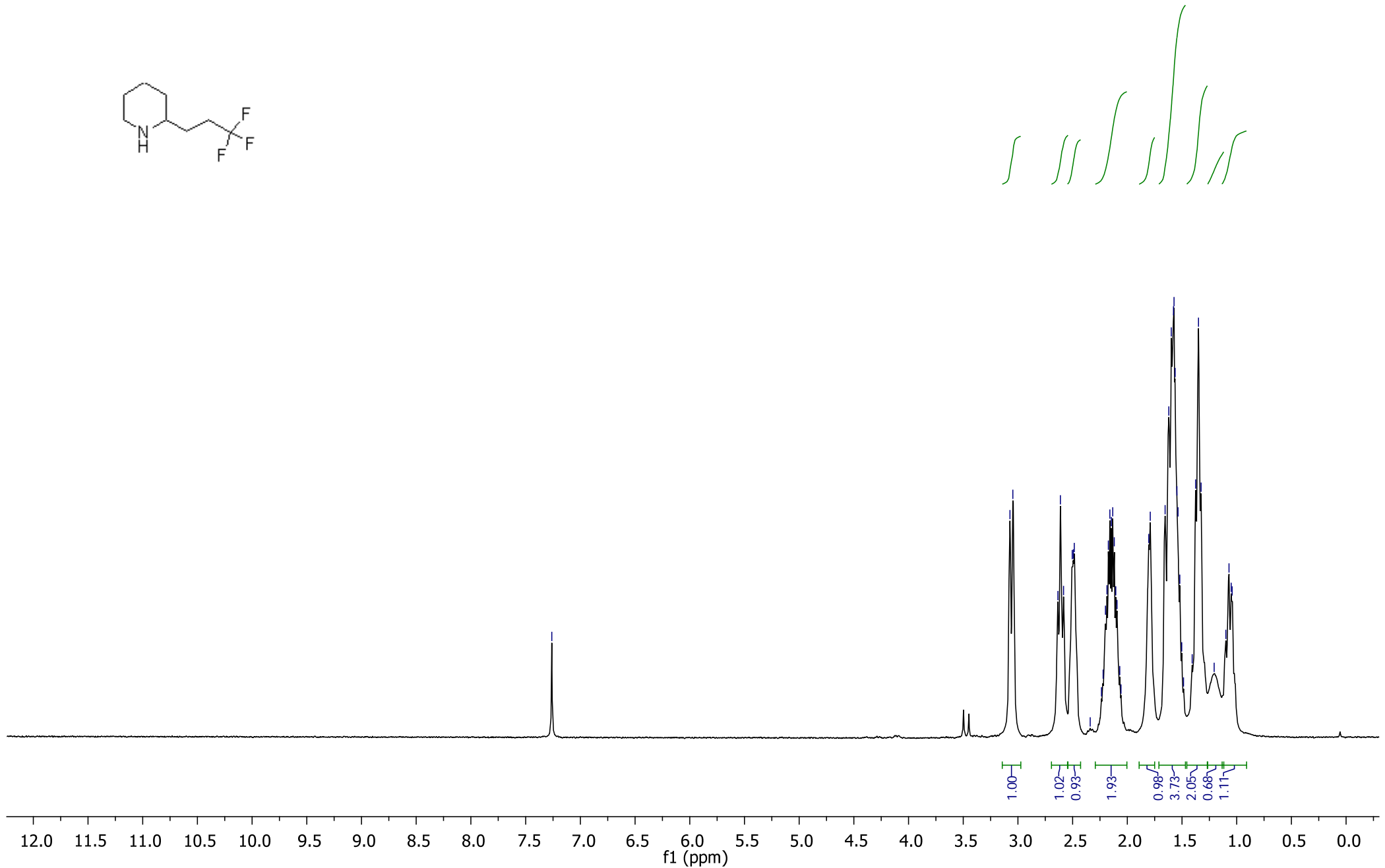
<smiles>FC(F)(F)CCC1CCCCN1</smiles>

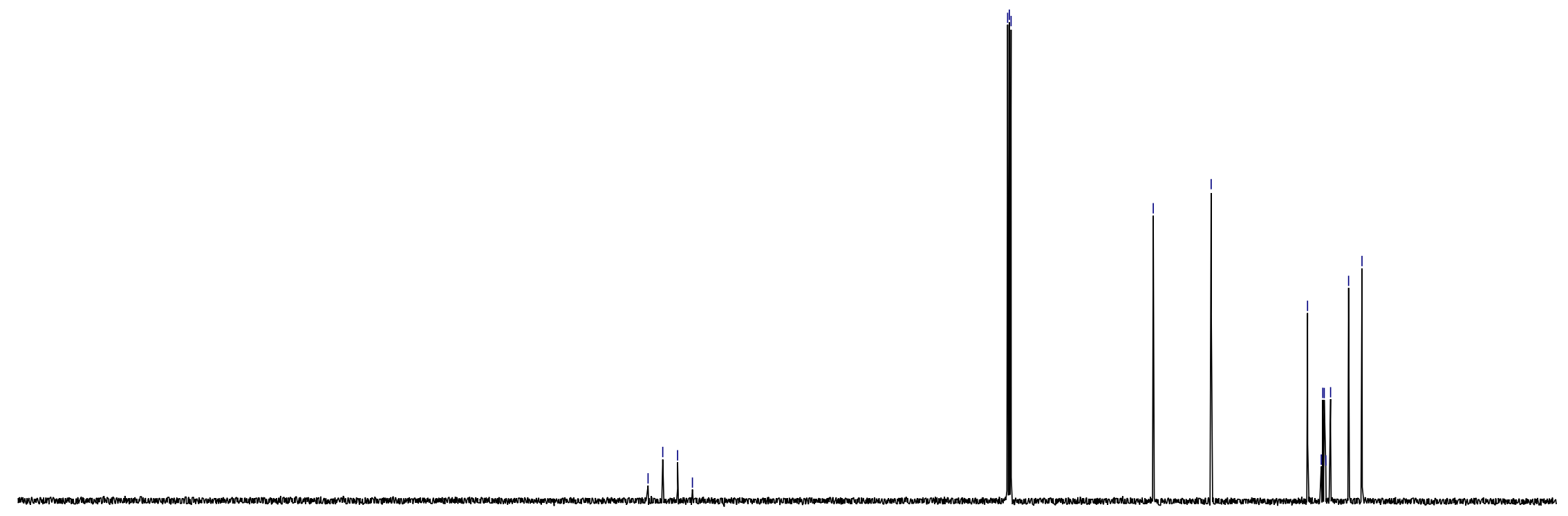


R1066471_F19 $\{\mathrm{H}\}$

$19 \mathrm{~F}-\{1 \mathrm{H}\}$
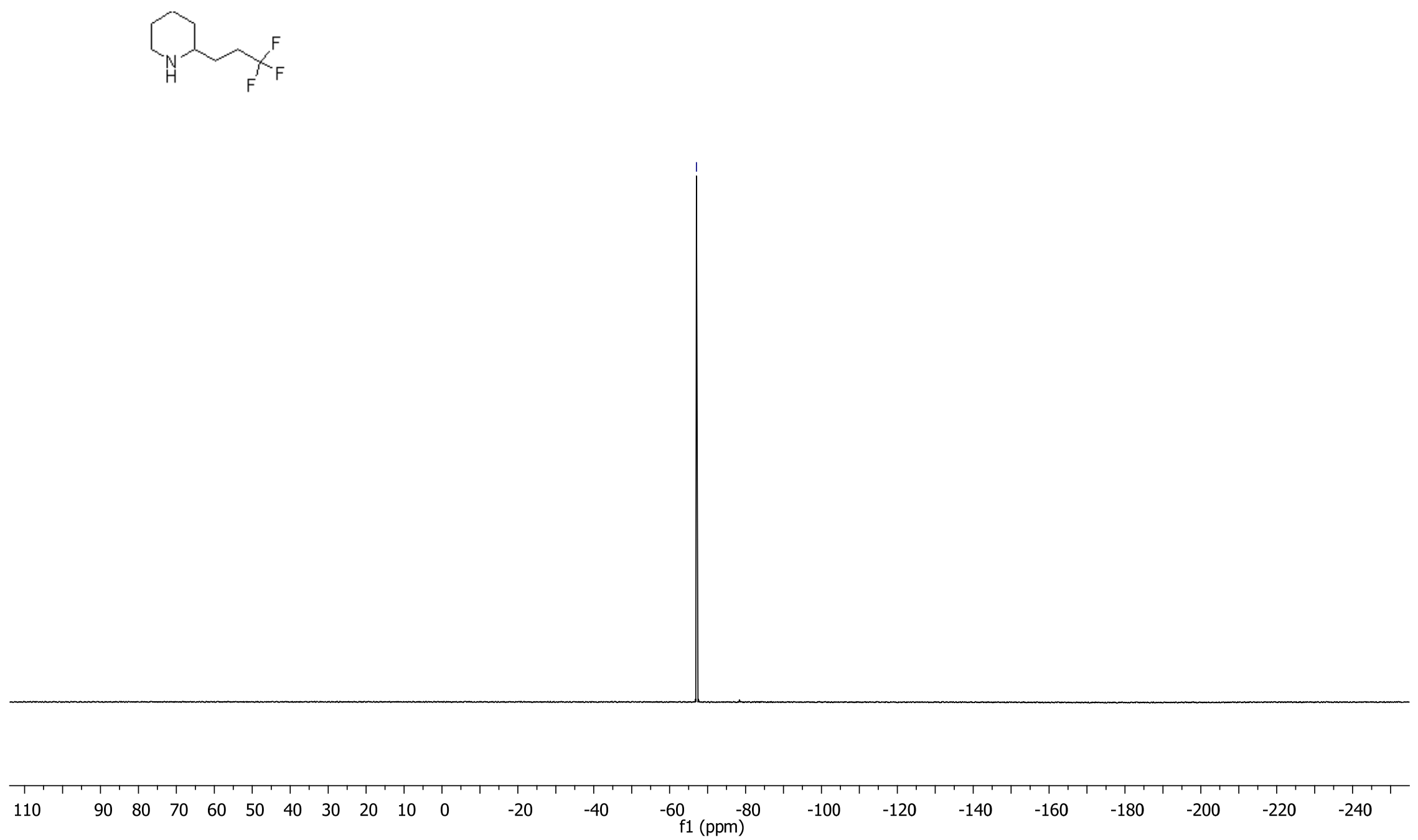


\section{Compound 21b}

R718525

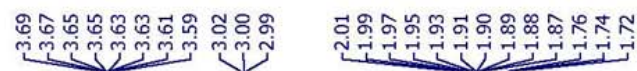

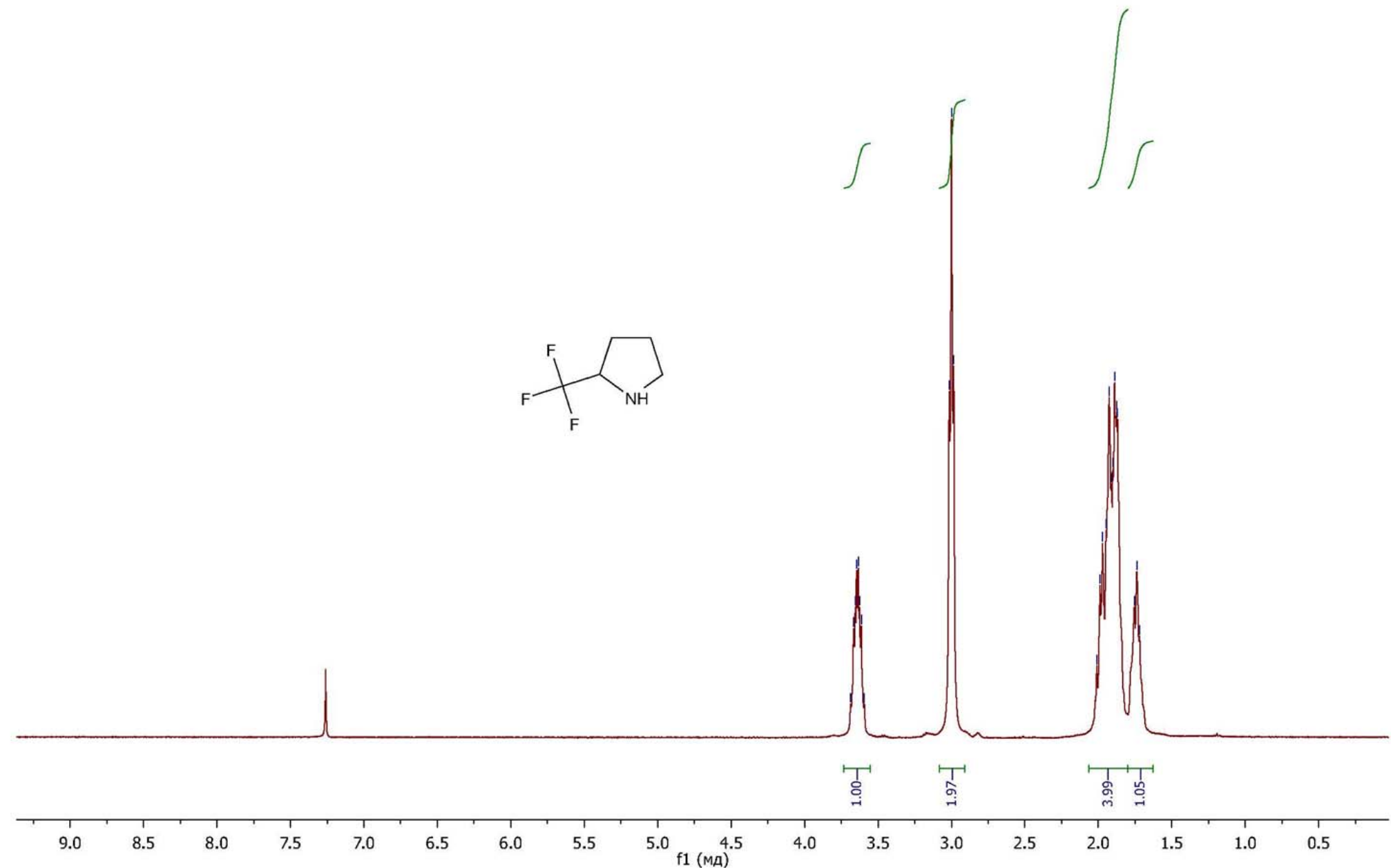


R718525_C13

$\mathrm{N}_{\mathrm{H}}^{\mathrm{F}}$

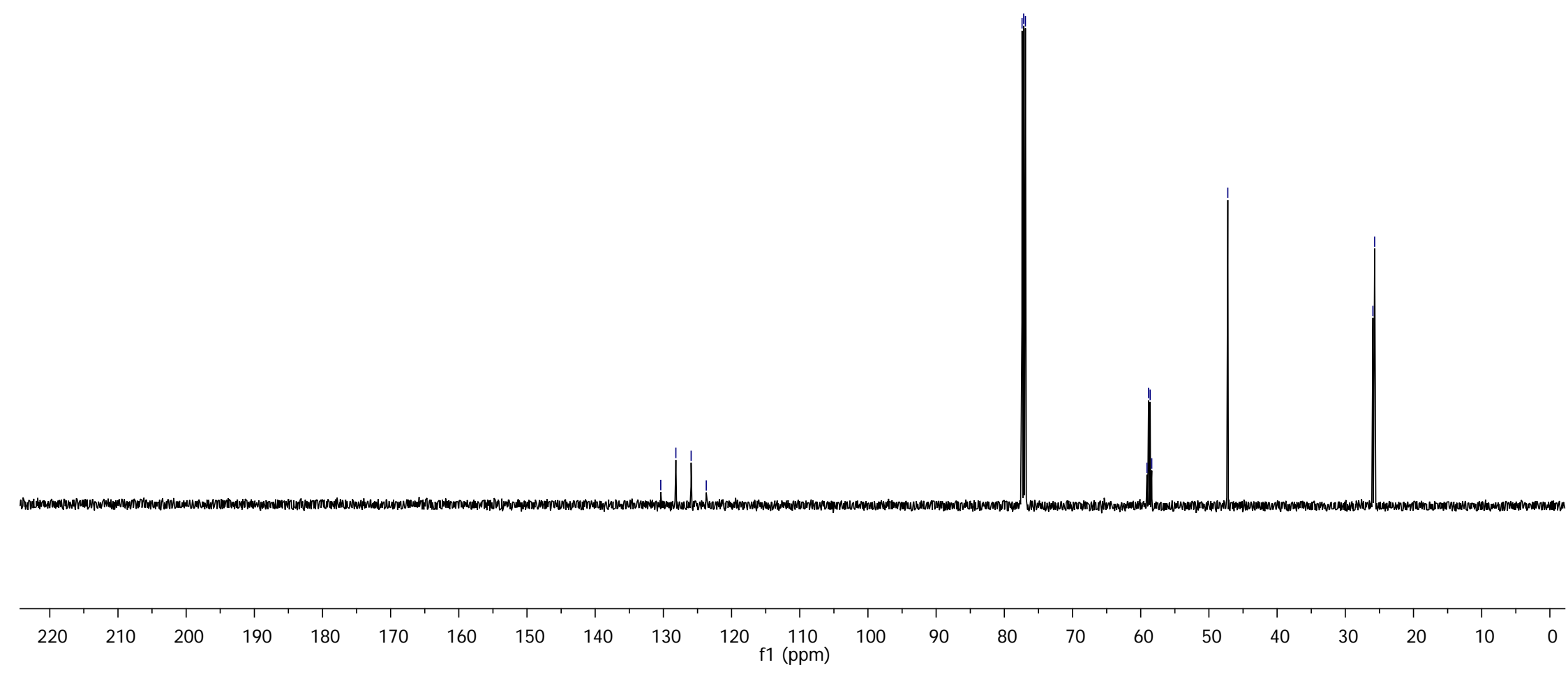




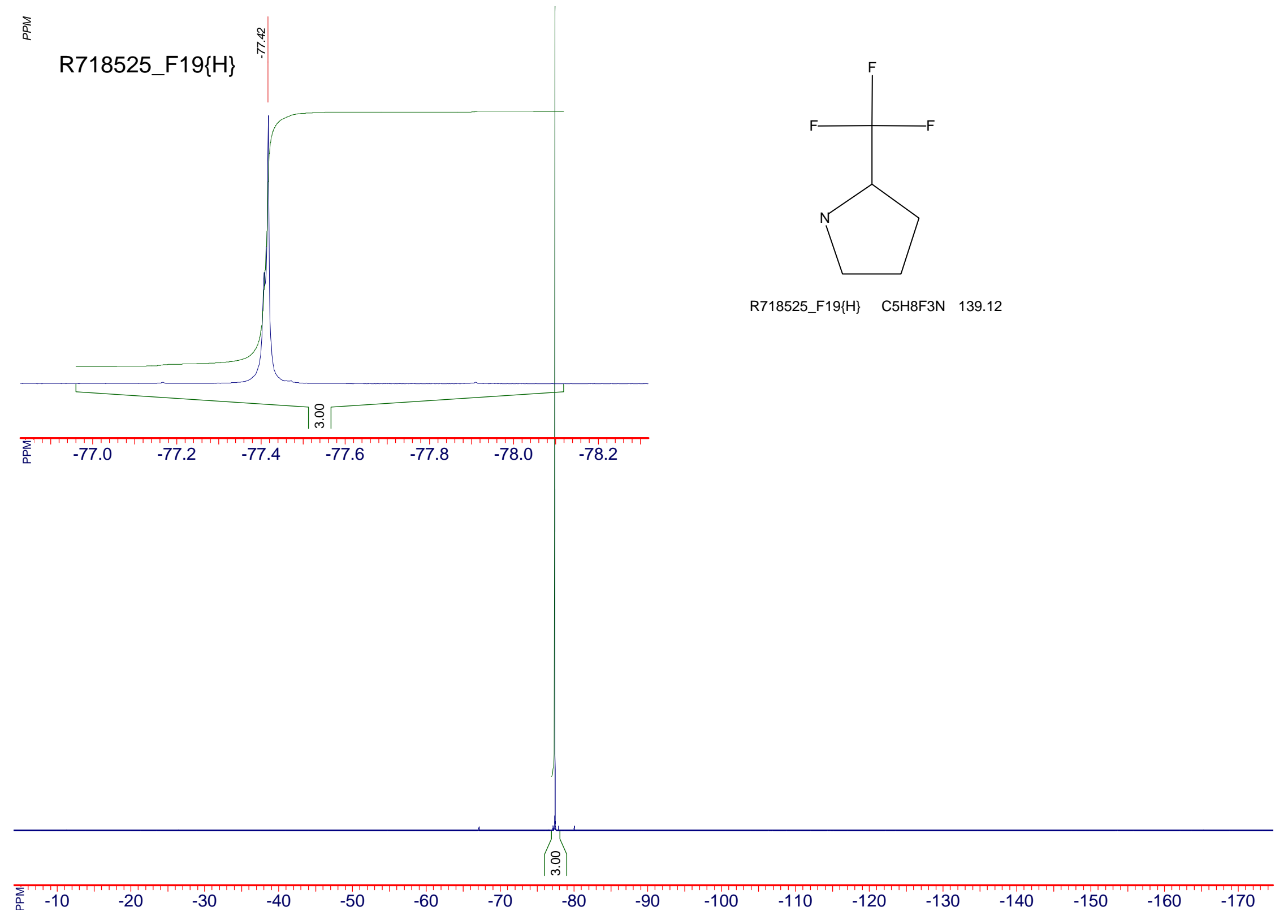


R718522_Mosher_F19
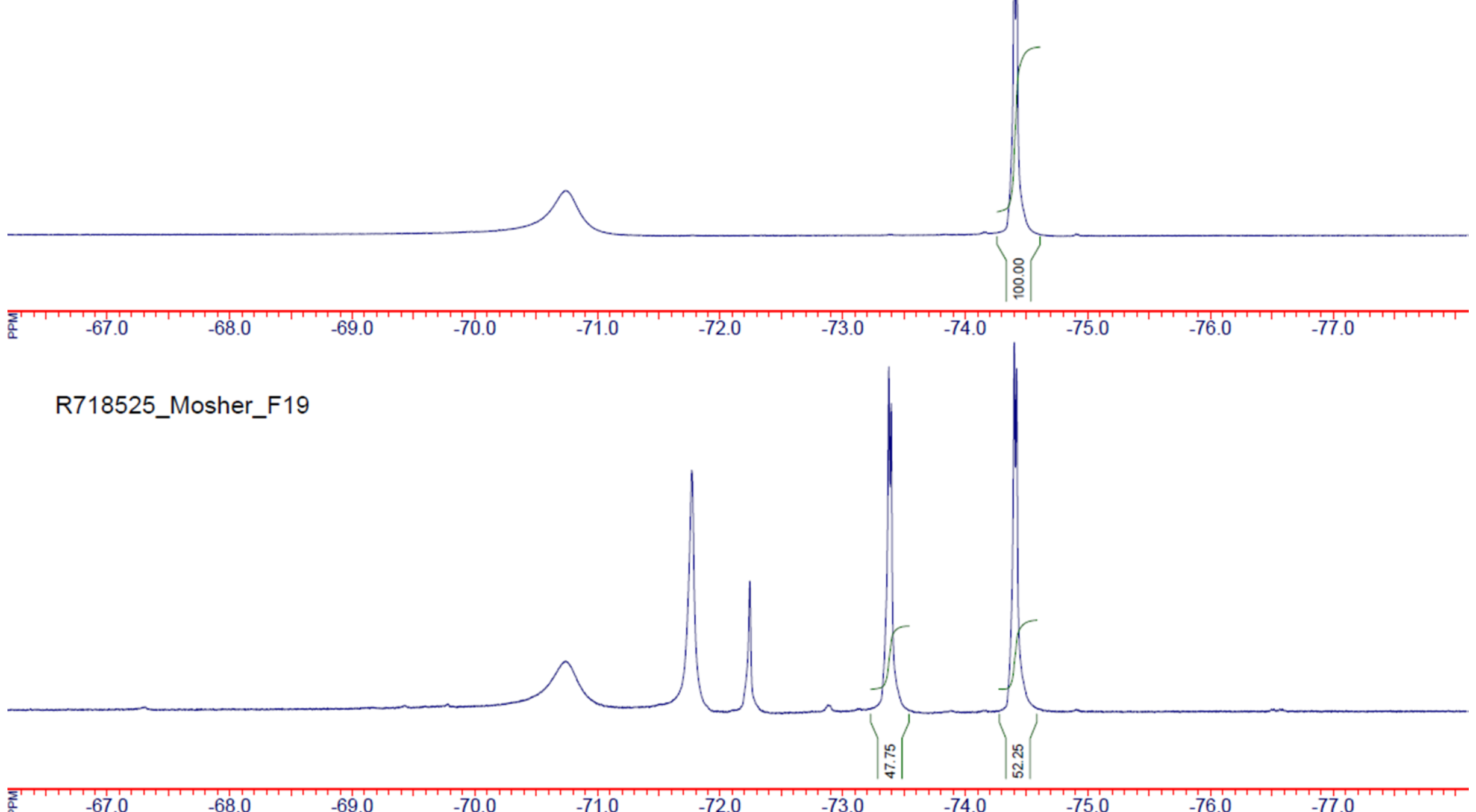


\section{Compound 29b}

R63689

रิ

fक्ष心

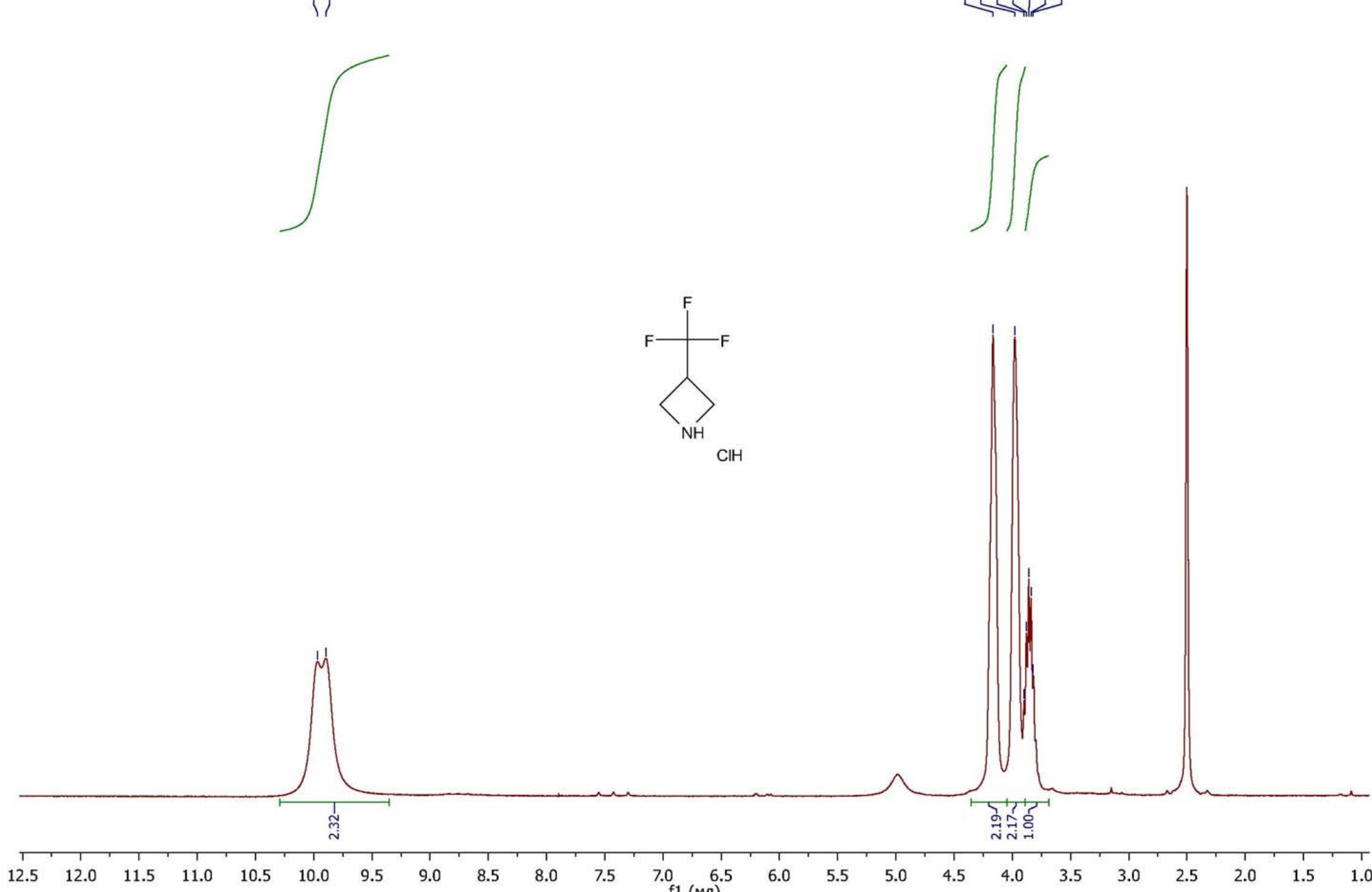


R636891 C13

$\infty \%$

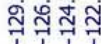

तार

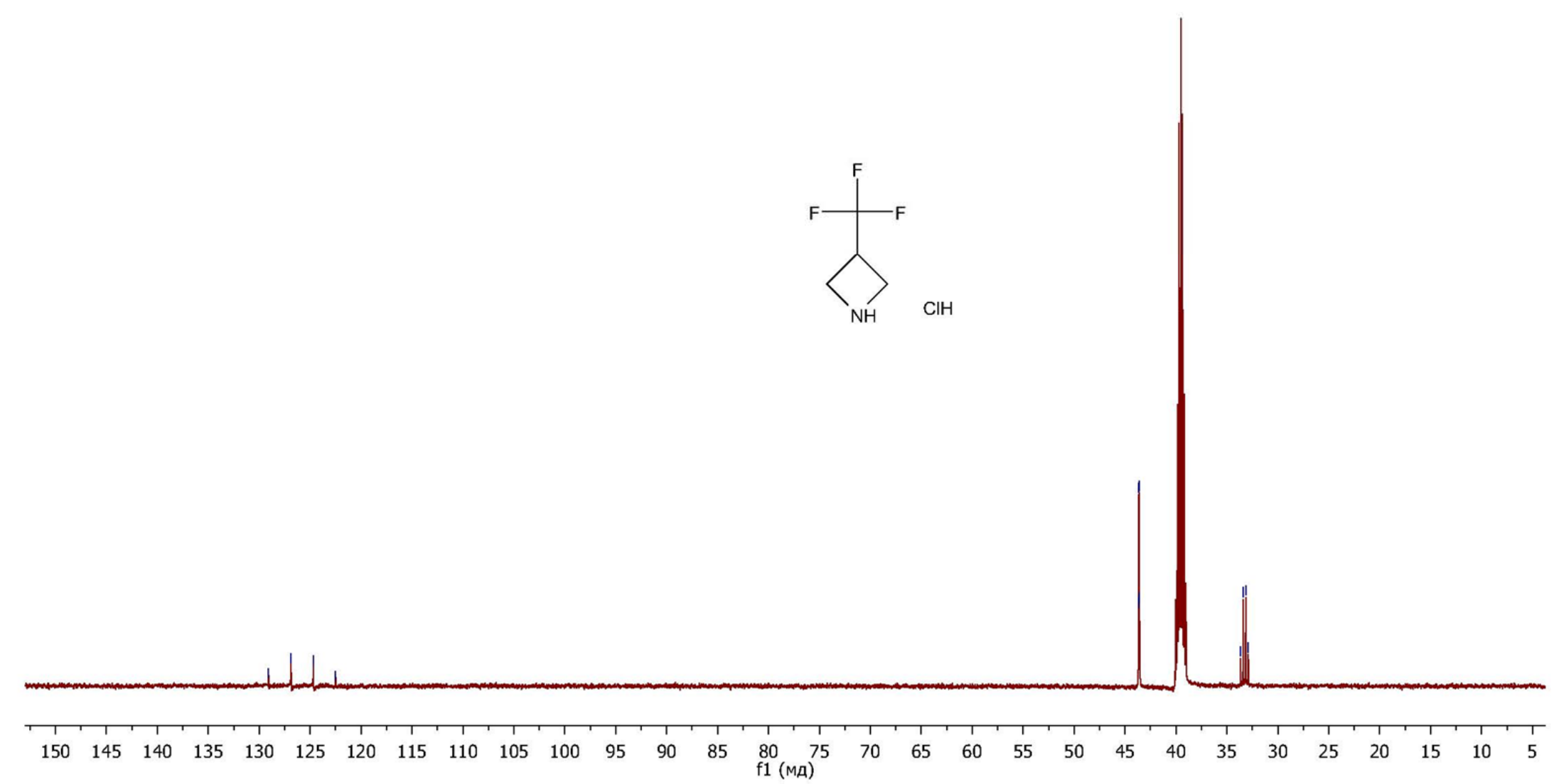



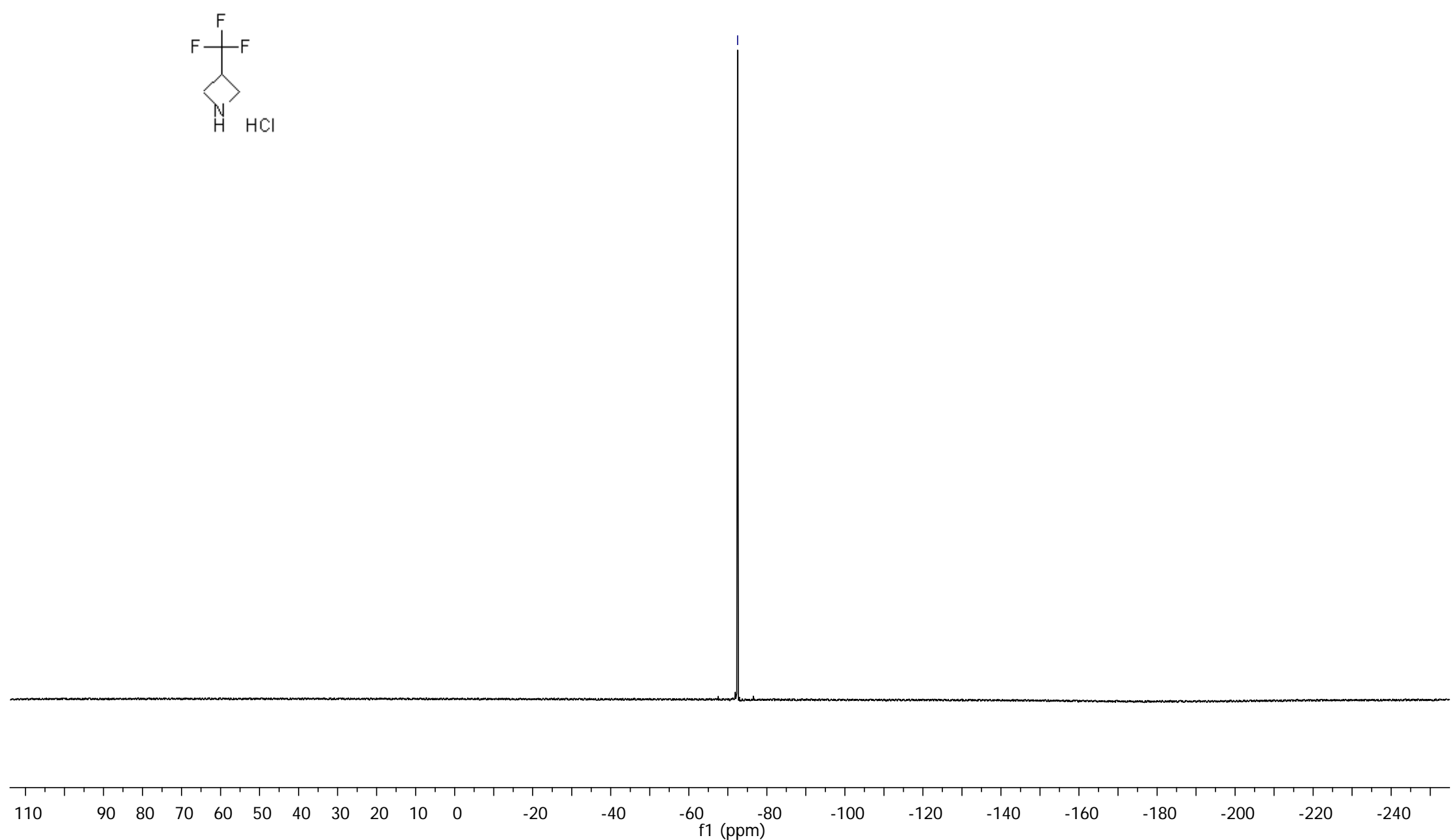


\section{Compound 30b}

R1471971

$$
\sum_{\substack{\mathrm{N} \\ \mathrm{H}}}^{\mathrm{CF}_{3}}
$$
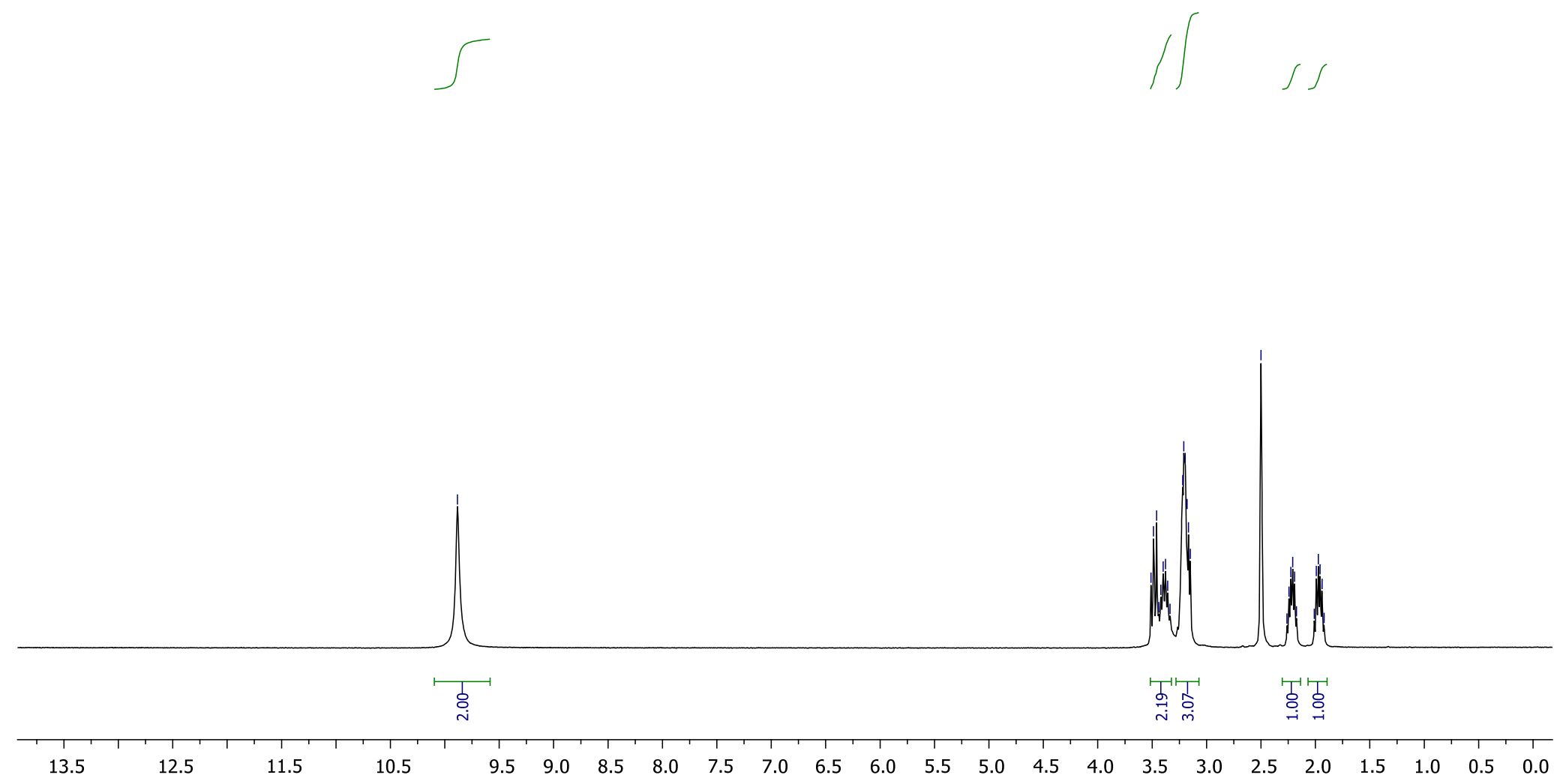


$$
\int_{\mathrm{H}}^{\mathrm{CF} \mathrm{F}_{3}}
$$

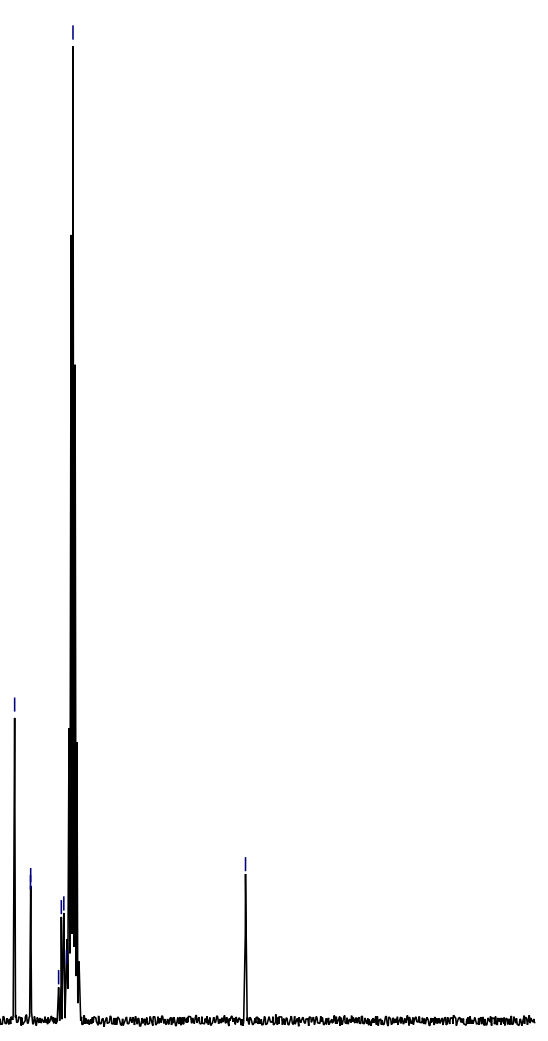


R115346_F19(H1)

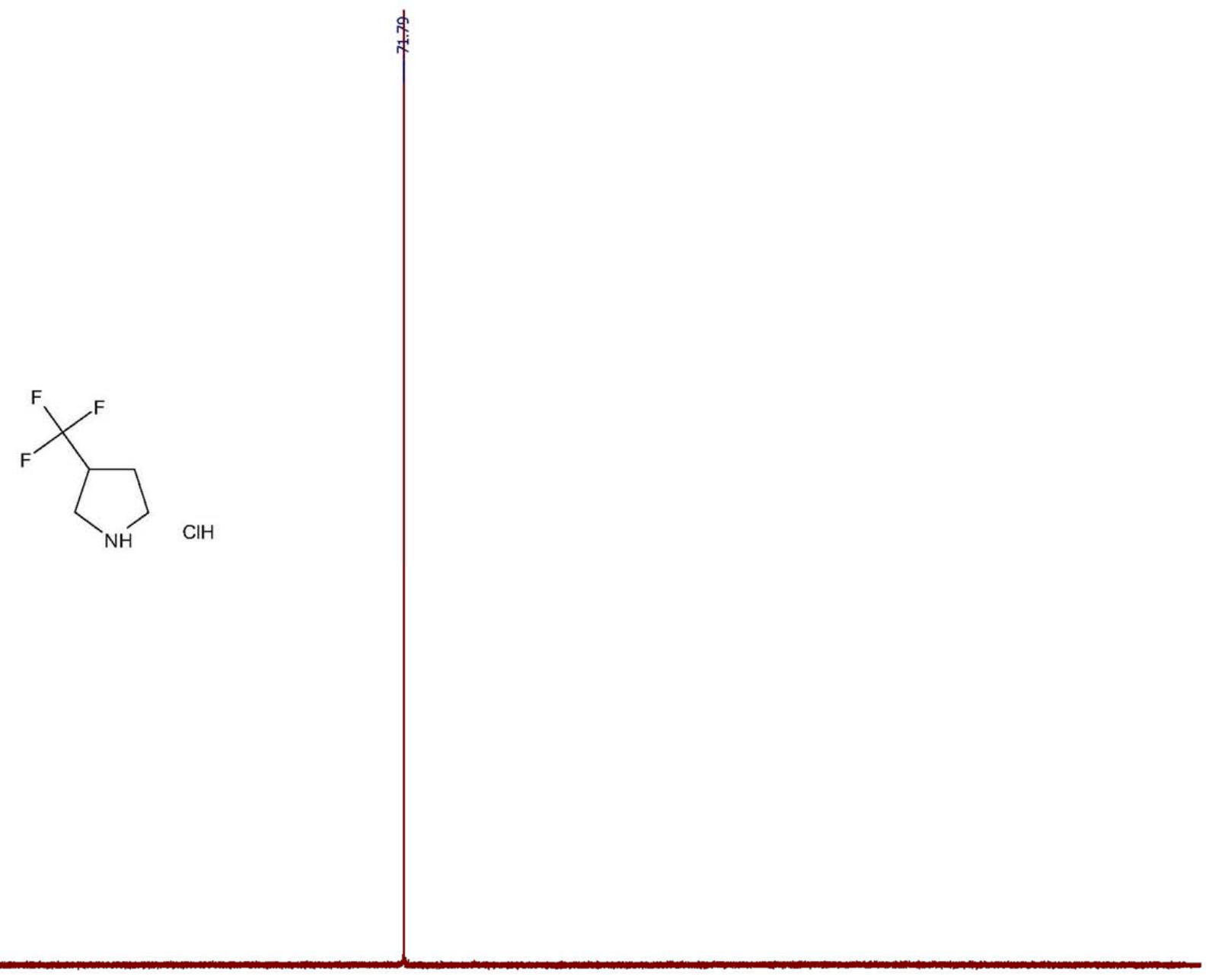

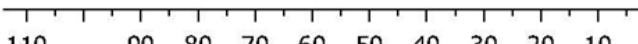

$\begin{array}{lllllllllllllll}90 & 80 & 70 & 60 & 50 & 40 & 30 & 20 & 10 & 0 & -20 & -40 & -60 & -80\end{array}$

$\begin{array}{llllllll}100 & -120 & -140 & -160 & -180 & -200 & -220 & -240\end{array}$

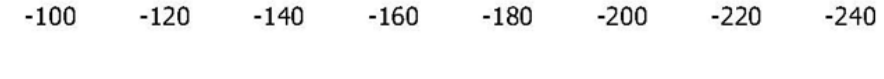




\section{Compound 31b}

R758881

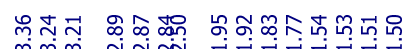

। 11 ।
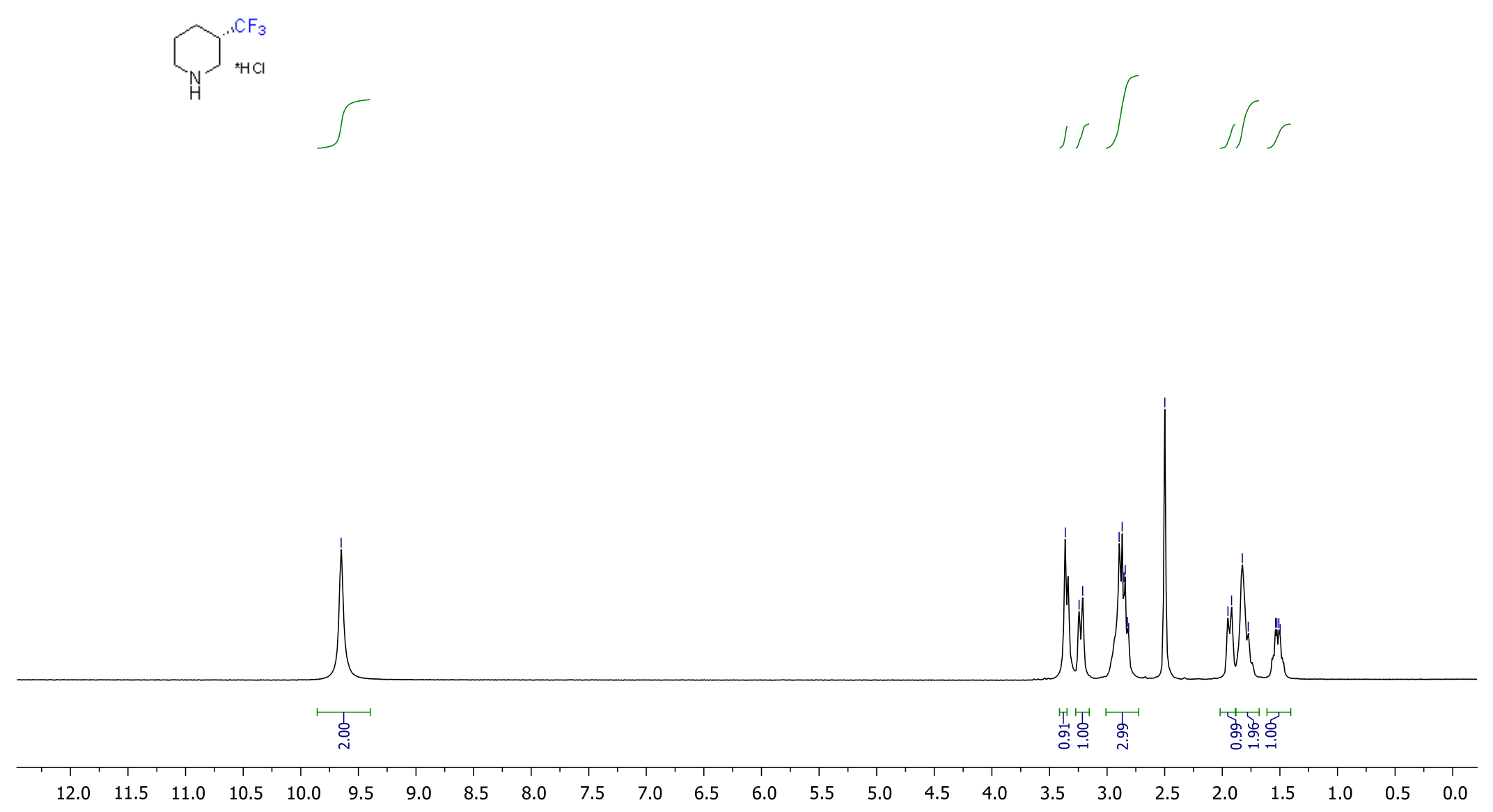
R758881_C13

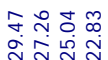

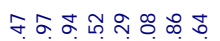

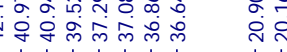

$\mathrm{C}_{\mathrm{H}}{ }^{. \mathrm{CF}_{3}}$

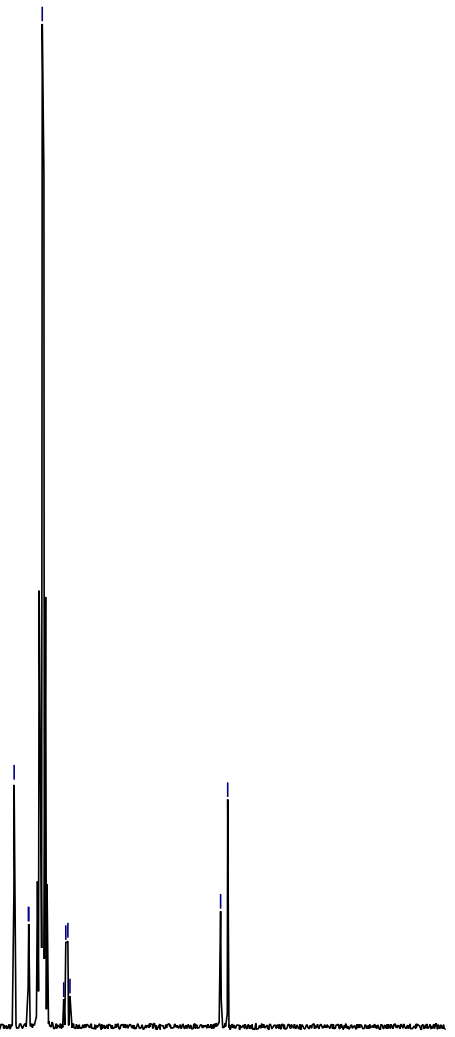

220

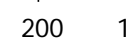


igrk3921_F19\{H\}
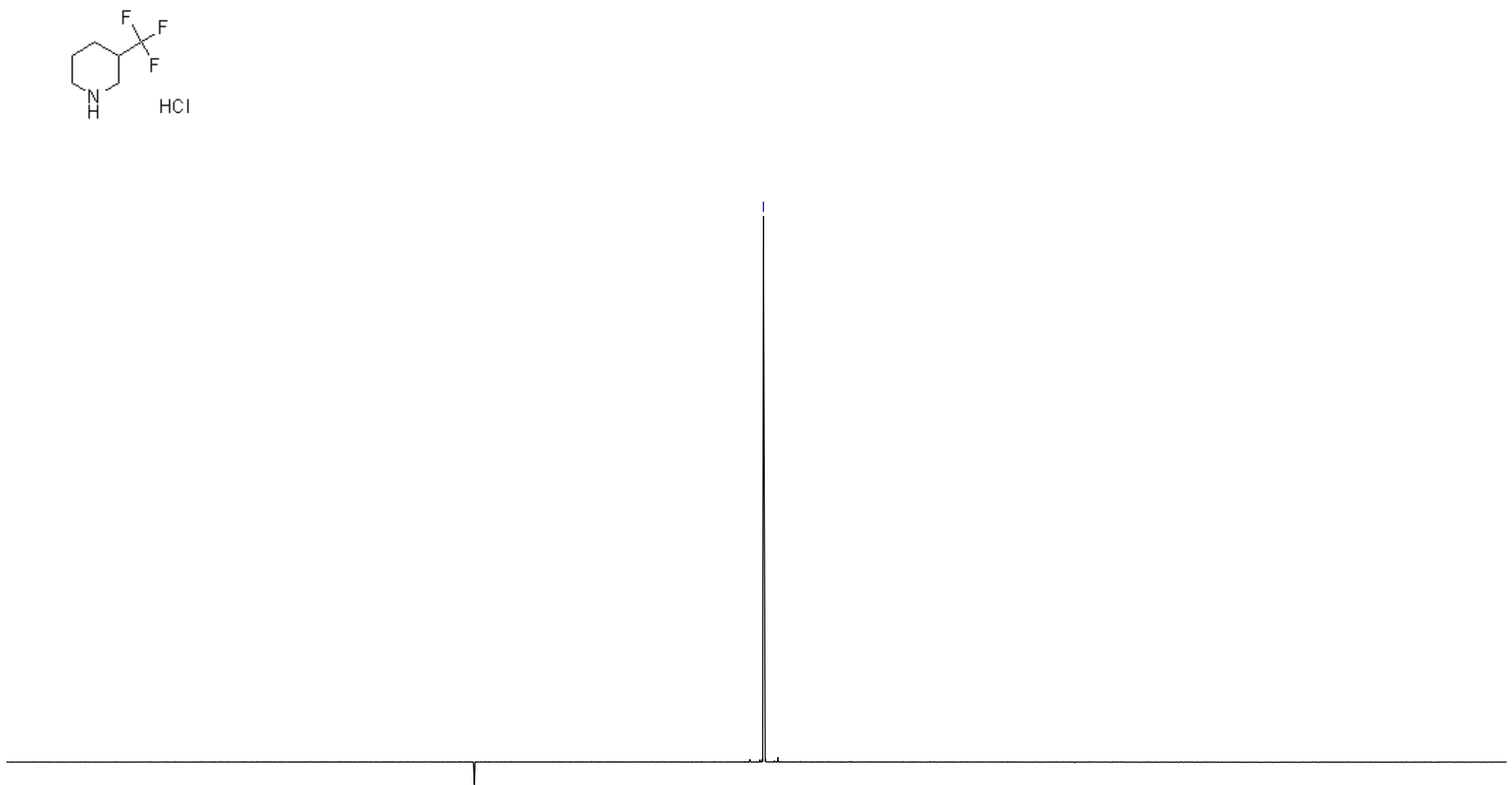

$\begin{array}{lllllllllllllllllllllllll}110 & 90 & 80 & 70 & 60 & 50 & 40 & 30 & 20 & 10 & 0 & -20 & -40 & -60 & -80 & -100 & -120 & -140 & -160 & -180 & -200 & -220 & -240\end{array}$




\section{Compound 39b}

R802983

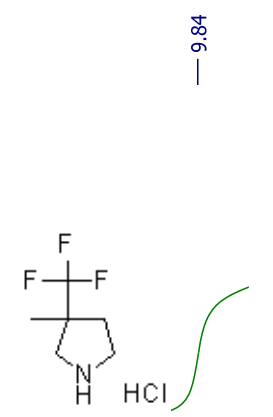

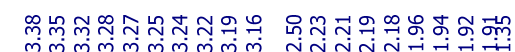

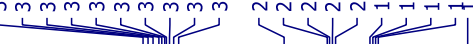
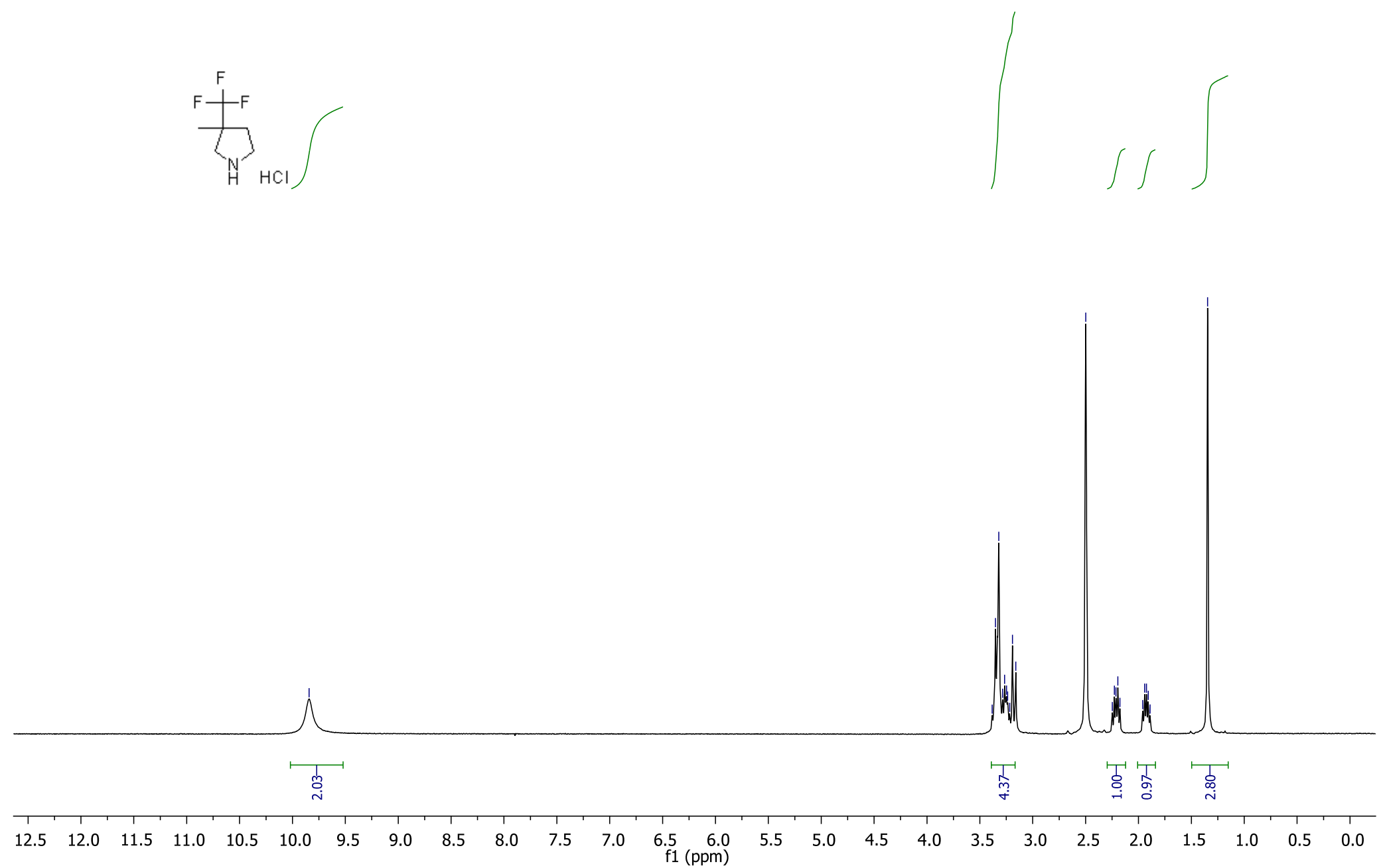
$\stackrel{\mathrm{F}+\mathrm{F}}{\mathrm{F}_{\mathrm{H}}}$

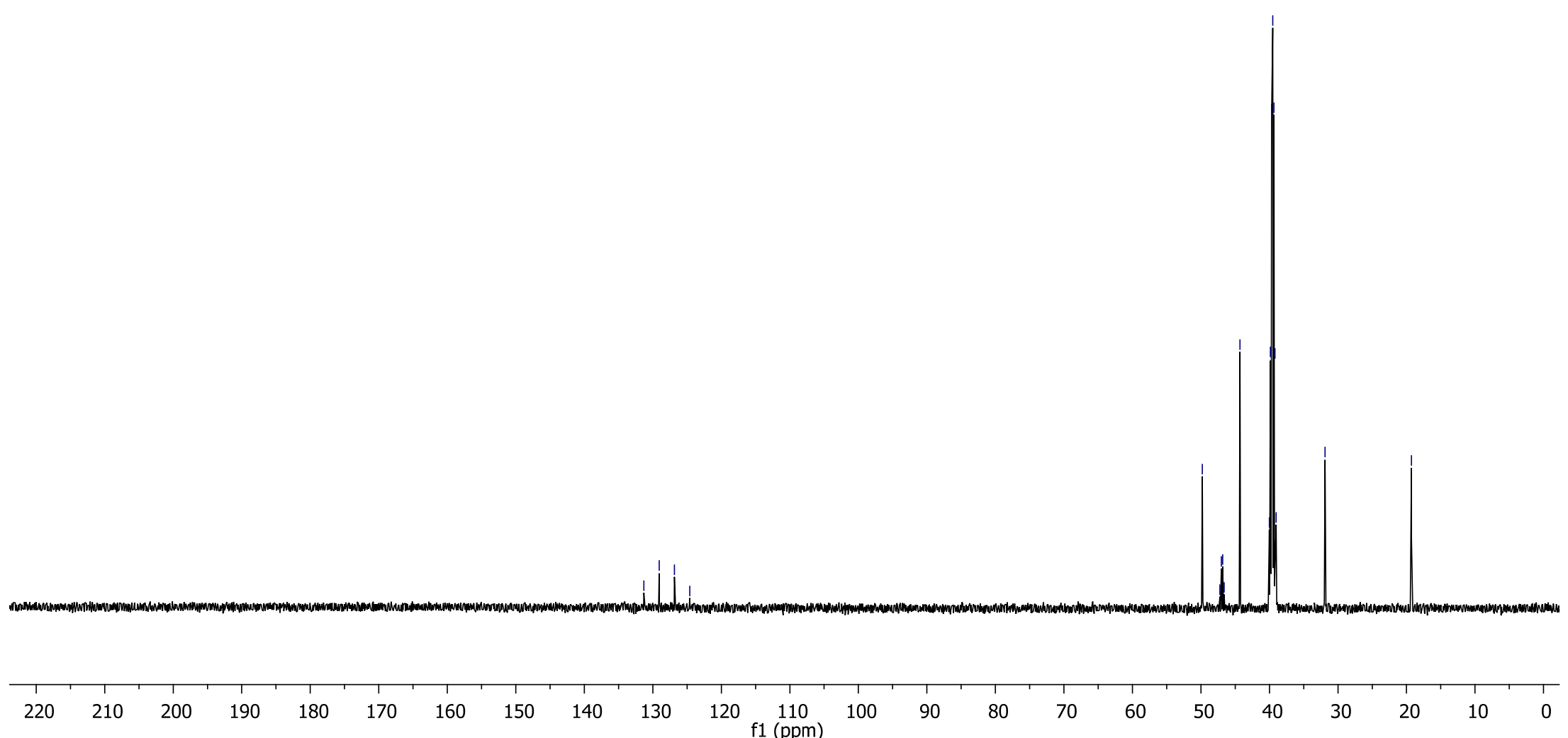




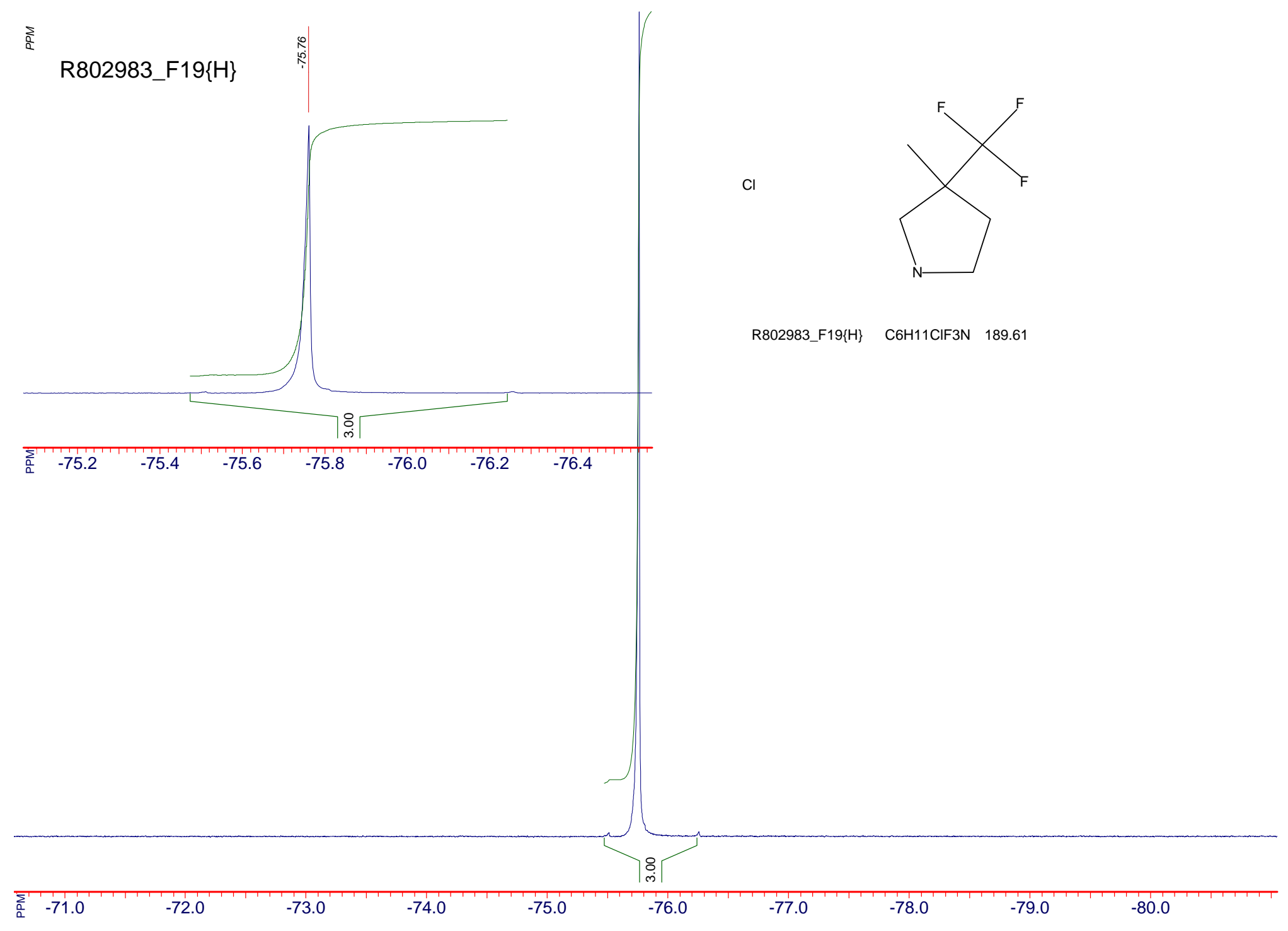




\section{Compound 40b}

$\sum_{\substack{\mathrm{N} \\ \mathrm{H}}} \mathrm{CF}_{\mathrm{HCl}}$

\&

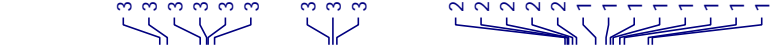
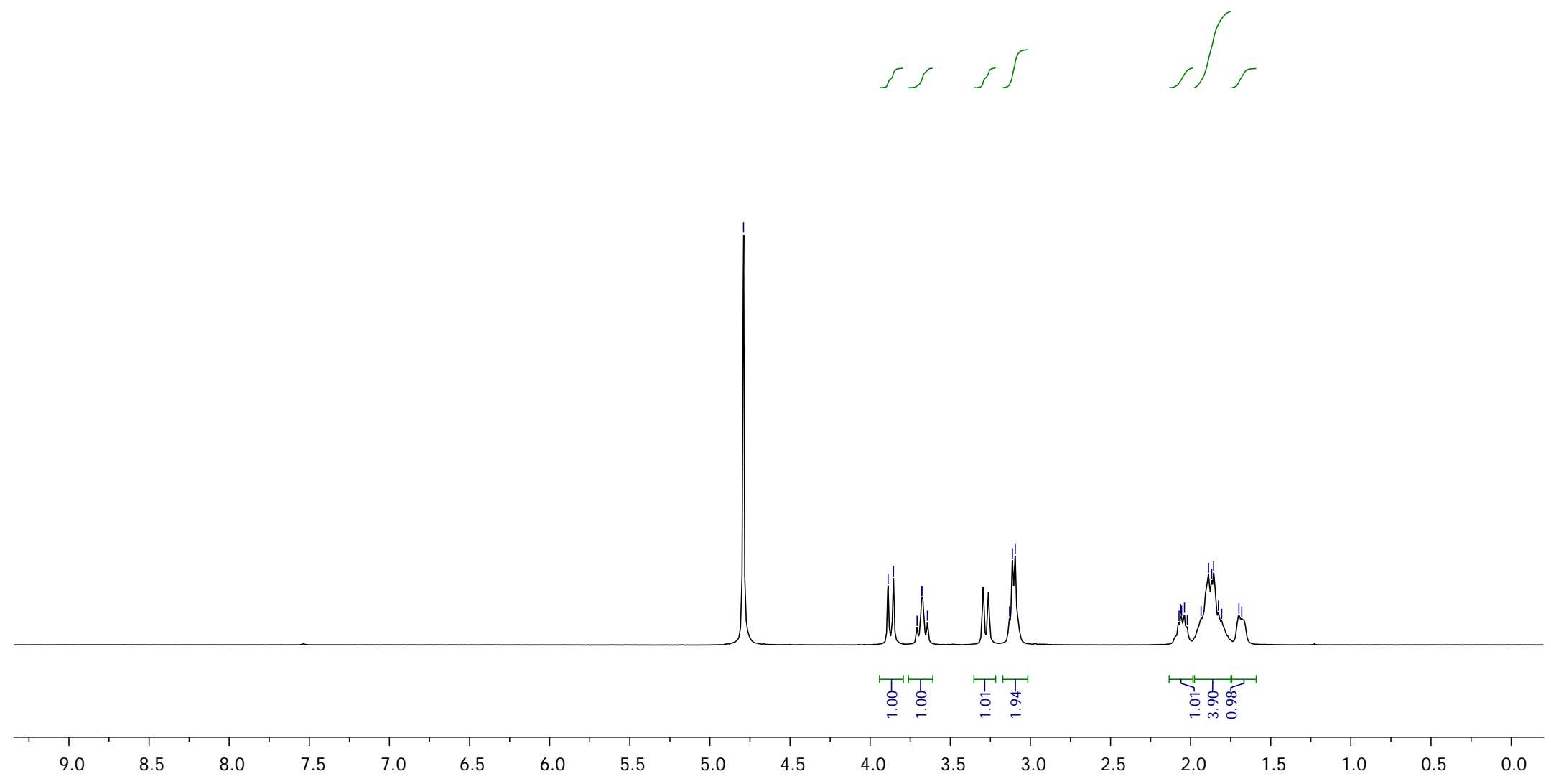


$\overbrace{\substack{N \\ \mathrm{CH}_{3}}}$

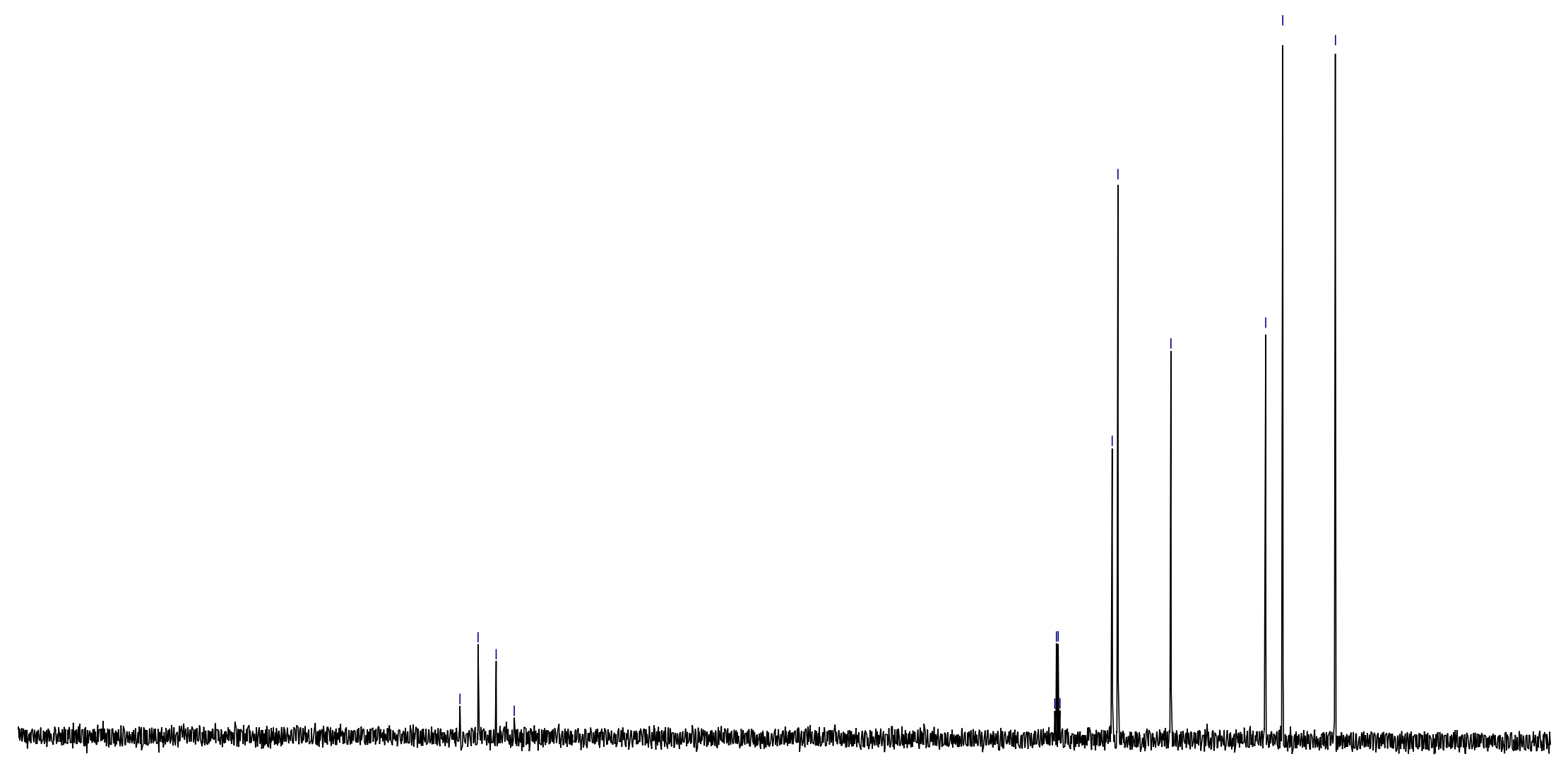

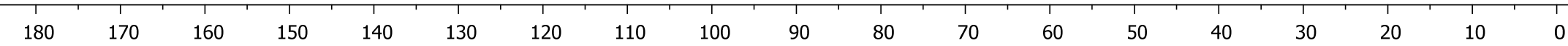


$\sum_{\substack{\mathrm{H} \\ \mathrm{H}}} \mathrm{CF}_{3}$

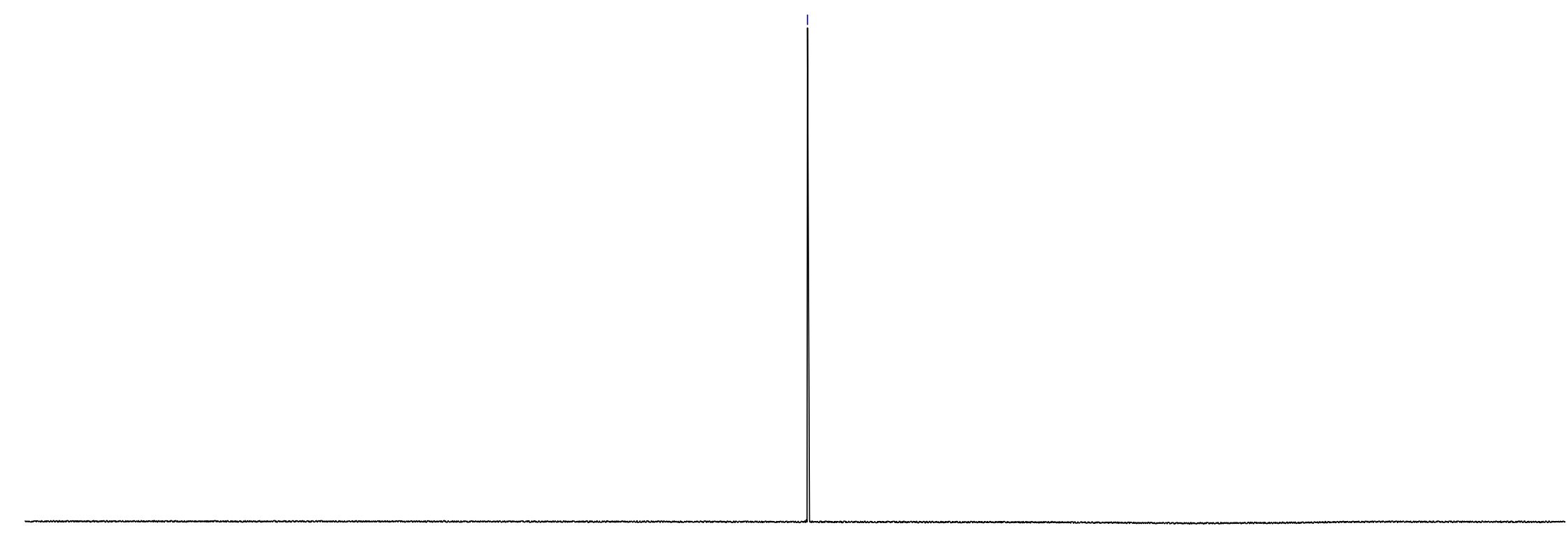

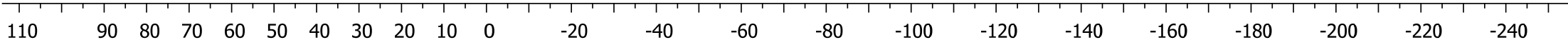




\section{Determination of the optical purity of compound (S)-21b}

Below, the chiral HPLC diagrams of $(S)$-21b (derived from $L$-Proline) and rac-21b (derived from rac-Proline) are given.
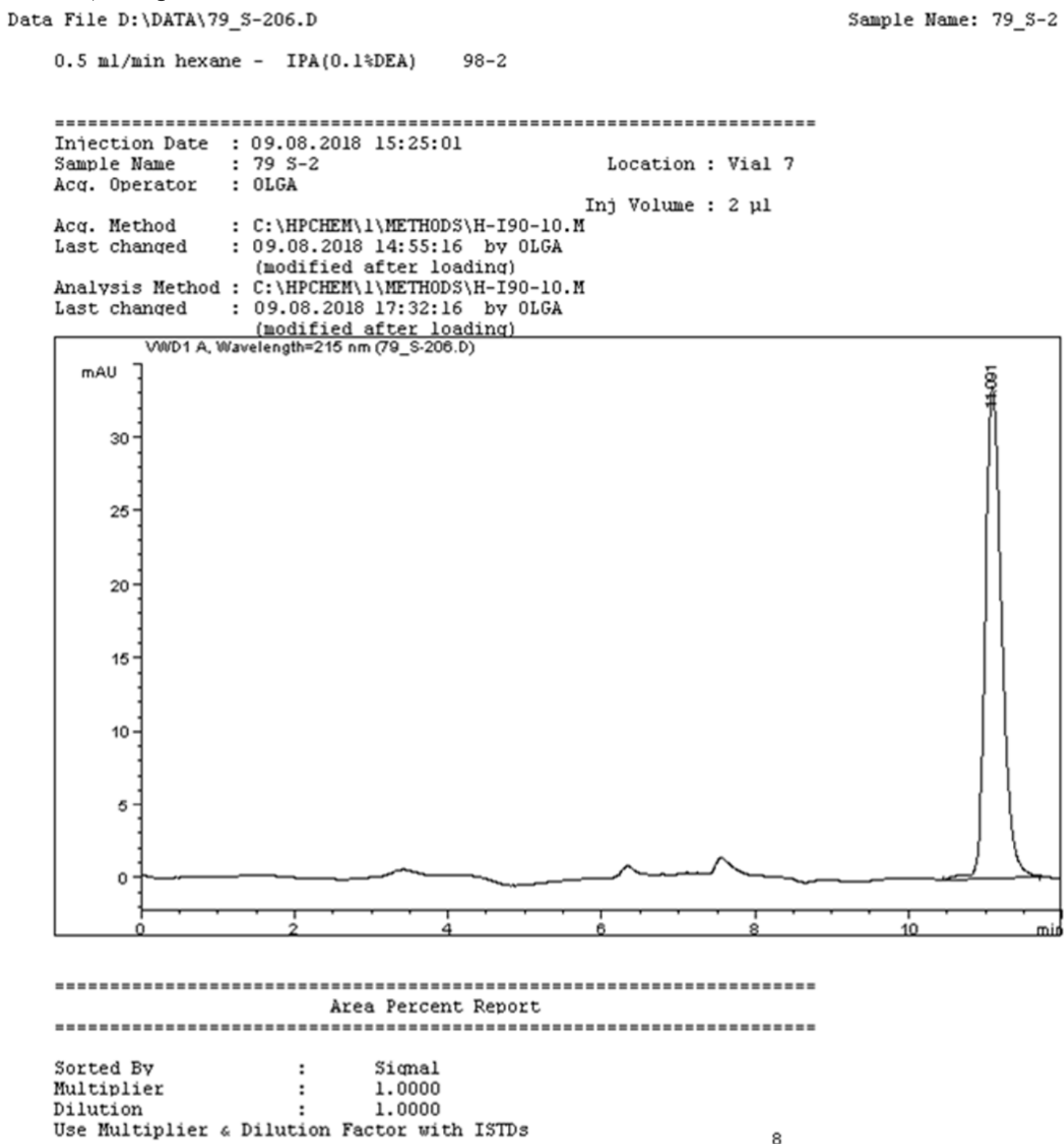

Use Multiplier \& Dilution Factor with ISTDs 8

Signal 1: VWD1 A, Wavelength=215 $\mathrm{nm}$

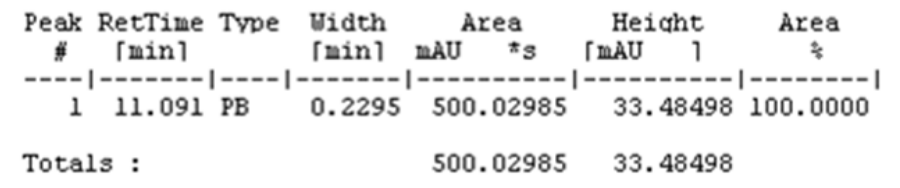

Results obtained with enhanced integrator!

** End of Report $t *$ * 

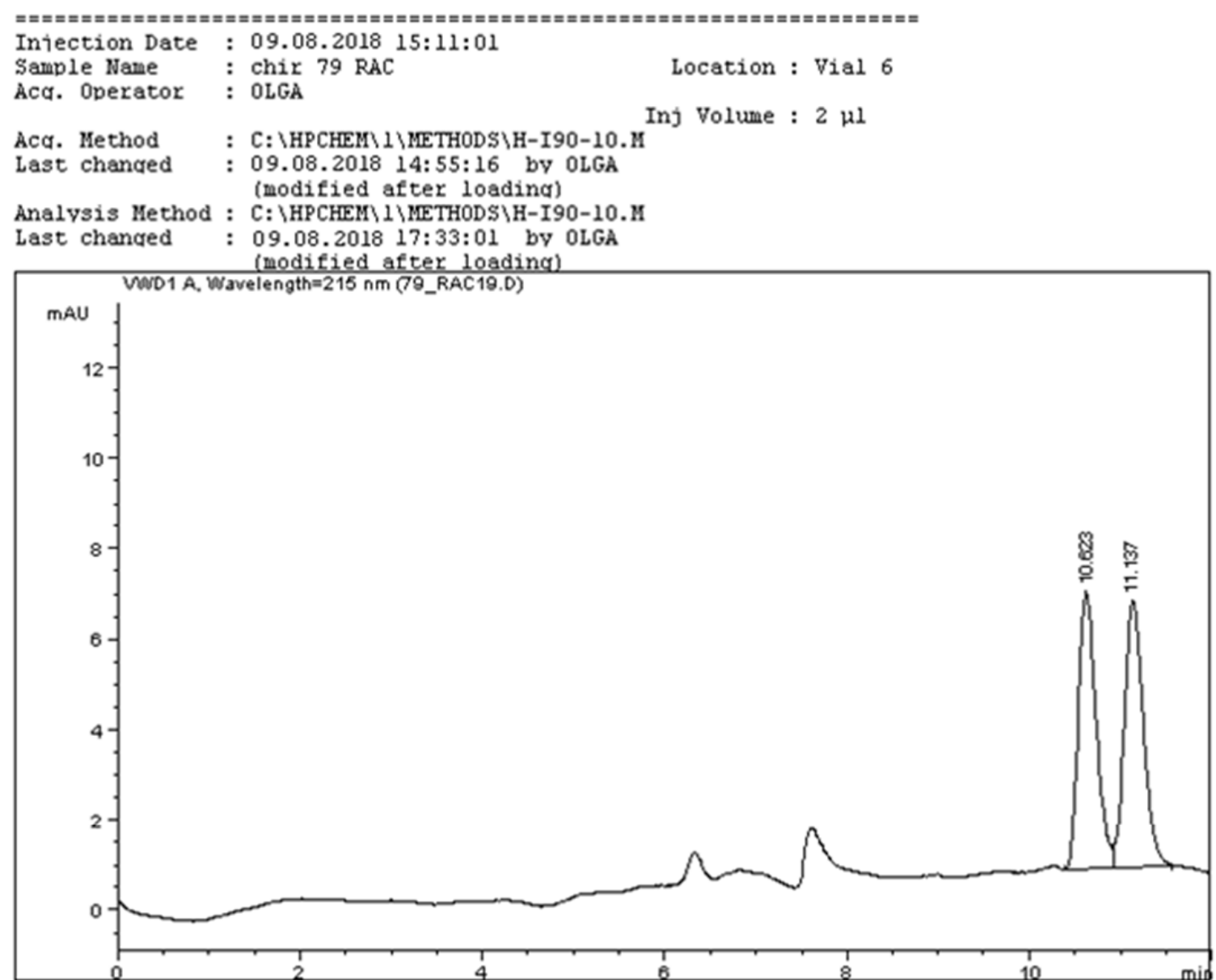

\section{Area Percent Report}

\begin{tabular}{|c|c|c|}
\hline Sorted By & : & Sicmal \\
\hline Multiplier & : & 1.0000 \\
\hline Dilution & : & 1.0000 \\
\hline
\end{tabular}

Use Multiplier \& Dilution Factor with ISTDs

Signal 1: VUD1 A, Wavelength=215 $\mathrm{nm}$

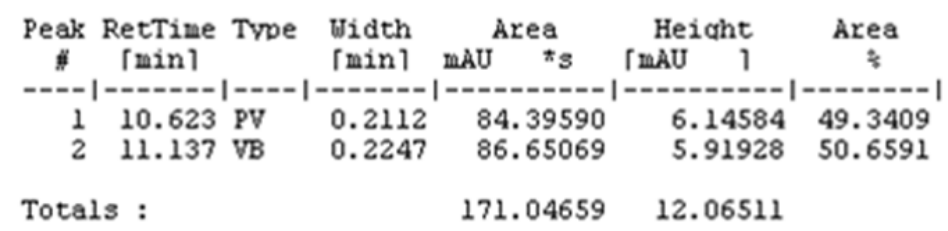

Results obtained with enhanced intecrator! 


\section{Crystallographic data (X-Ray)}

Crystals of compounds 12a, 22a, 43a, 29b and 39b suitable for X-Ray diffractional studies were obtained by a low evaporation of a solution of listed compounds in methanol. Diffraction data were collected at room temperature on a Bruker SMART APEX II diffractometer with graphitemonochromated Mo K $\alpha$ radiation $(\lambda=0.71073 \AA)$ operating in the $\omega$ scans mode. These data were integrated using the program SAINT while the absorption correction was carried out using the program SADABS.

The structures were solved by direct methods and refined by the full-matrix least-squares technique in the anisotropic approximation for non-hydrogen atoms using the Bruker SHELXTL program package. Crystallographic data for all structures in this paper have been deposited at Cambridge Crystallographic Data Centre. Copies of the data can be obtained, free of charge, on application to CCDC, 12 Union Road, Cambridge CB21EZ, UK, (fax: +44-(0)1223-336033 or e-mail: deposit@ccdc.cam.ac.uk).

Compound 12a (1889642)

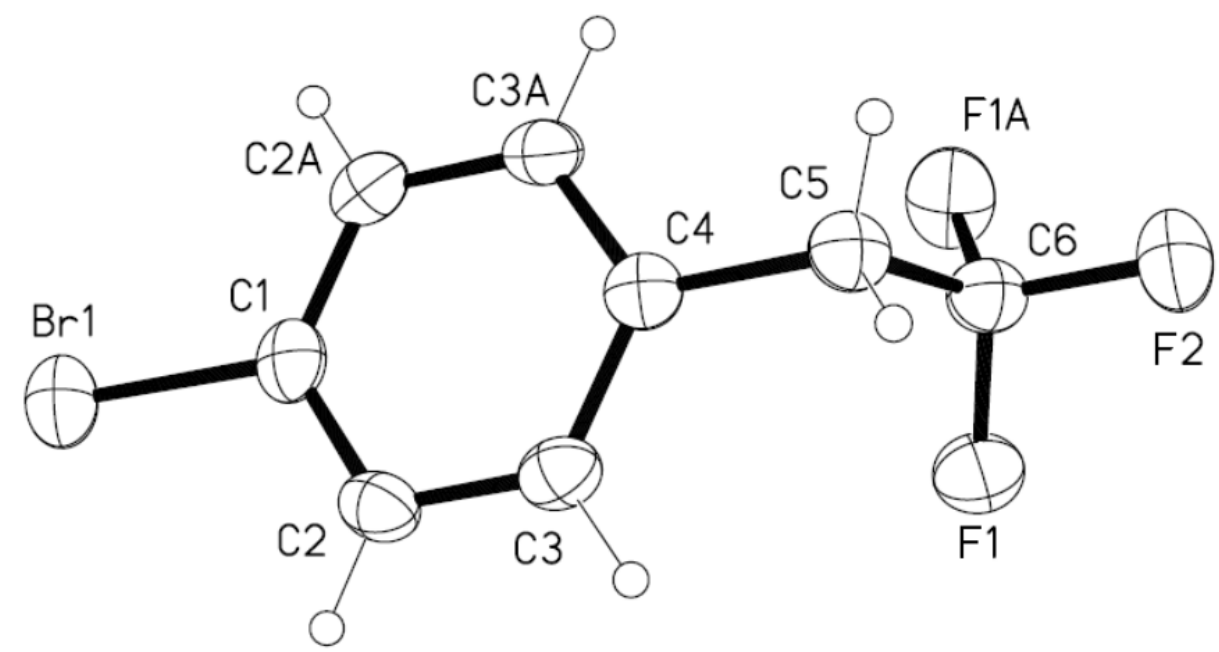

Figure S1. Structure of compound 12a according to X-ray diffraction data. Ellipsoids are shown at $30 \%$ probability level.

\section{Crystal data and structure refinement parameters:}

$\begin{array}{lc}\text { _name_xr98 } & \\ \text { _audit_creation_method } & \text { SHELXL-2016/4 } \\ \text { _-shelx_SHELXL_version_number } 2016 / 4 \\ \text { _-chemical_melting_point } & 333 \\ \text { _cheming } & \\ \text { _chemical_formula_moiety } & \mathrm{C}_{8} \mathrm{H}_{6} \mathrm{BrF}_{3} \\ \text { _chemical_formula_weight } & 239.04\end{array}$




\begin{tabular}{|c|c|}
\hline cell_length_a & $19.766(4)$ \\
\hline cell_length_b & $9.0401(15)$ \\
\hline cell_length_c & $4.6202(8)$ \\
\hline cell_angle_alpha & 90 \\
\hline cell_angle_beta & 90 \\
\hline cell_angle_gamma & 90 \\
\hline cell_volume & $825.6(3)$ \\
\hline cell_formula_units_Z & 4 \\
\hline _cell_measurement_tempera & ature $173(2)$ \\
\hline cell_measurement_reflns_u & used $\quad 686$ \\
\hline cell_measurement_theta_m & $\operatorname{nin} \quad 3.05$ \\
\hline cell_measurement_theta_m & $\operatorname{nax} \quad 22.85$ \\
\hline _exptl_crystal_description & 'block' \\
\hline _exptl_crystal_colour & 'colourless' \\
\hline exptl_crystal_density_diffrn & rn 1.923 \\
\hline _exptl_crystal_F_000 & 464 \\
\hline exptl_crystal_size_max & 0.330 \\
\hline exptl_crystal_size_mid & 0.200 \\
\hline exptl_crystal_size_min & 0.130 \\
\hline _exptl_absorpt_coefficient_r & $\mathrm{mu} \quad 4.966$ \\
\hline _shelx_estimated_absorpt_T & $\Gamma$ min 0.291 \\
\hline _shelx_estimated_absorpt_T & $\Gamma \_\max \quad 0.565$ \\
\hline _exptl_absorpt_correction_ty & type multi-scan \\
\hline _exptl_absorpt_correction_T & T_min 0.51 \\
\hline _exptl_absorpt_correction_T & $T_{-} \max 0.57$ \\
\hline _exptl_absorpt_process_deta & ails SADABS \\
\hline _diffrn_ambient_temperature & re $173(2)$ \\
\hline _diffrn_radiation_wavelengt & th $\quad 0.71073$ \\
\hline _diffrn_radiation_type & $\mathrm{MoK} \backslash \mathrm{a}$ \\
\hline _diffrn_radiation_source & 'sealed tube' \\
\hline _diffrn_radiation_monochrol & omator 'graphite' \\
\hline _diffrn_measurement_device & e_type 'CCD area detector' \\
\hline _diffrn_measurement_metho & od 'omega scans' \\
\hline _diffrn_reflns_number & 4919 \\
\hline _diffrn_reflns_av_unetI/netI & 0.0309 \\
\hline _diffrn_reflns_av_R_equival & lents 0.0431 \\
\hline _diffrn_reflns_limit_h_min & -24 \\
\hline _diffrn_reflns_limit_h_max & 23 \\
\hline _diffrn_reflns_limit_k_min & -11 \\
\hline _diffrn_reflns_limit_k_max & 10 \\
\hline _diffrn_reflns_limit_1_min & -5 \\
\hline diffrn_reflns_limit_1_max & 5 \\
\hline _diffrn_reflns_theta_min & 2.061 \\
\hline _diffrn_reflns_theta_max & 25.998 \\
\hline _diffrn_reflns_theta_full & 25.242 \\
\hline diffrn_measured_fraction_t & theta_max 1.000 \\
\hline
\end{tabular}


_diffrn_measured_fraction_theta_full 1.000

_diffrn_reflns_Laue_measured_fraction_max 1.000

_diffrn_reflns_Laue_measured_fraction_full 1.000

_diffrn_reflns_point_group_measured_fraction_max 1.000

_diffrn_reflns_point_group_measured_fraction_full 1.000

_reflns_number_total 866

_reflns_number_gt 713

_reflns_threshold_expression 'I $>2 \backslash \mathrm{s}(\mathrm{I})^{\prime}$

Compound 22a (1889643)

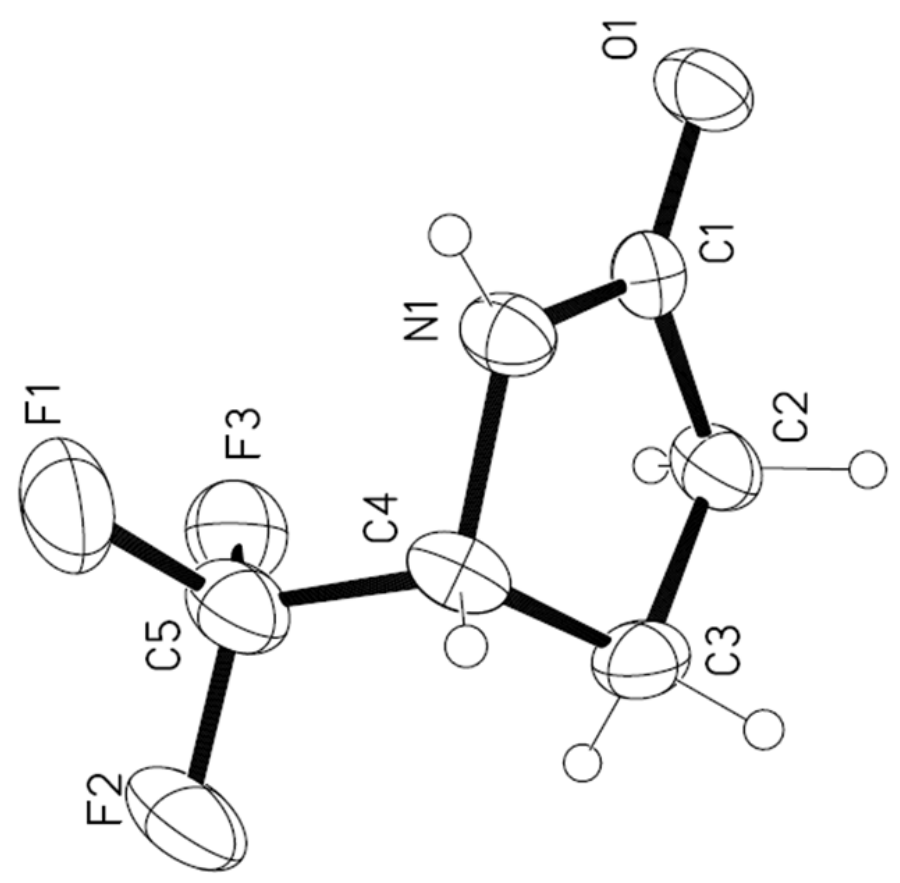

Figure S2. Structure of compound 22a according to X-ray diffraction data. Ellipsoids are shown at $30 \%$ probability level.

\section{Crystal data and structure refinement parameters:}

\begin{tabular}{|c|c|}
\hline _audit_creation_method & 'SHELXL-2016/4' \\
\hline _shelx_SHELXL_version_nu & ber '2016/4' \\
\hline chemical_formula_moiety & $\mathrm{C}_{5} \mathrm{H}_{6} \mathrm{~F}_{3} \mathrm{NO}$ \\
\hline chemical_formula_weight & 153.11 \\
\hline space_group_crystal_system & monoclinic \\
\hline space_group_IT_number & 5 \\
\hline _space_group_name_H-M_al & 'C 2' \\
\hline _space_group_name_Hall & 'C 2y' \\
\hline cell_length_a & $00(4)$ \\
\hline cell_length_b & $6(4)$ \\
\hline cell_length_c & $81(6)$ \\
\hline cell_angle_alpha & \\
\hline cell_angle_beta & $3.12(2)$ \\
\hline cell_angle_gamma & 90 \\
\hline
\end{tabular}


cell_volume

633.0(6)

_cell_formula_units_Z 4

_cell_measurement_temperature 123(2)

_cell_measurement_reflns_used 262

_cell_measurement theta_min 3.75

_cell_measurement_theta_max 22.27

_exptl_crystal_description 'plate'

_exptl_crystal_colour 'colourless'

_exptl_crystal_density_diffrn 1.607

_exptl_crystal_F_000 312

_exptl_crystal_size_max $\quad 0.500$

_exptl_crystal_size_mid $\quad 0.220$

_exptl_crystal_size_min 0.030

_exptl_absorpt_coefficient_mu 0.169

_shelx_estimated_absorpt_T_min 0.920

_shelx_estimated_absorpt_T_max 0.995

_exptl_absorpt_correction_type numerical

_exptl_absorpt_correction_T_min 0.60

_exptl_absorpt_correction_T_max 1.00

_exptl_absorpt_process_details SADABS

_diffrn_ambient_temperature 123(2)

_diffrn_radiation_wavelength 0.71073

_diffrn_radiation_type MoKla

_diffrn_radiation_source_sealed tube'

_diffrn_radiation_monochromator 'graphite'

_diffrn_measurement_device_type 'CCD area detector'

_diffrn_measurement_method_omega scans'

_diffrn_reflns_number 3297

_diffrn_reflns_av_unetI/netI 0.0396

_diffrn_reflns_av_R_equivalents 0.0318

_diffrn_reflns_limit_h_min -11

_diffrn_reflns_limit_h_max 11

_diffrn_reflns_limit_k_min -8

_diffrn_reflns_limit_k_max 8

_diffrn_reflns_limit_1_min -11

_diffrn_reflns_limit_1_max 12

diffrn_reflns_theta_min 2.113

_diffrn_reflns_theta_max 25.472

_diffrn_reflns_theta_full 25.242

_diffrn_measured_fraction_theta_max 1.000

_diffrn_measured_fraction_theta_full 1.000

_diffrn_reflns_Laue_measured_fraction_max 1.000

_diffrn_reflns_Laue_measured_fraction_full 1.000

_diffrn_reflns_point_group_measured_fraction_max 0.959

_diffrn_reflns_point_group_measured_fraction_full 0.963

_reflns_number_total 1133

_reflns_number_gt 923

_reflns_threshold_expression 'I $>2 \backslash \mathrm{s}(\mathrm{I})^{\prime}$

_reflns_Friedel_coverage $\quad 0.765$

_reflns_Friedel_fraction_max 0.911

_reflns_Friedel_fraction_full 0.919 


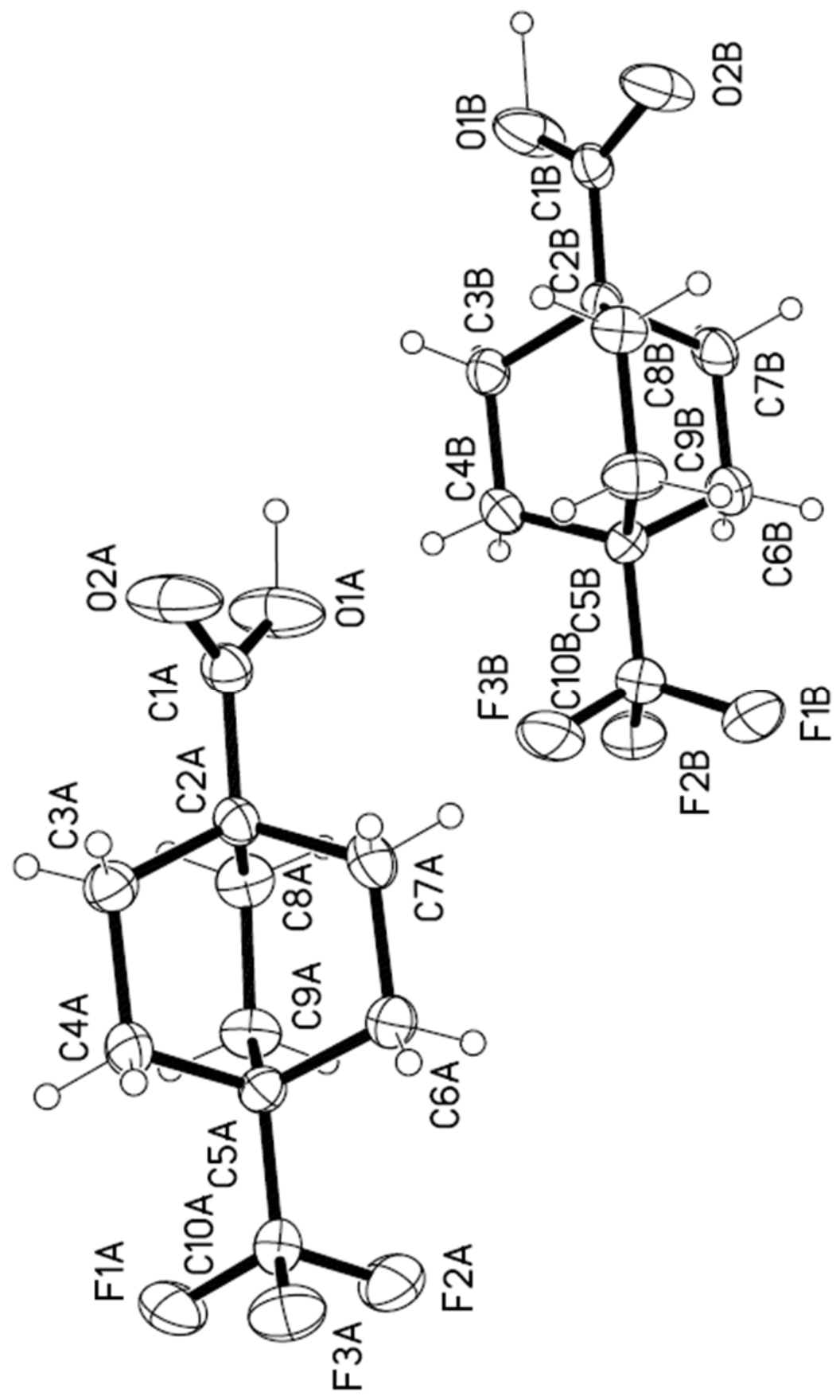

Figure S3. Structure of compound 43a according to X-ray diffraction data. Ellipsoids are shown at $30 \%$ probability level.

\section{Crystal data and structure refinement parameters:}

\begin{tabular}{|c|c|}
\hline audit_creation_method & SHELXL-2016/4' \\
\hline shelx_SHELXL_version_numb & ber $\quad 2016 / 4$ \\
\hline chemical_formula_moiety & $\mathrm{C}_{10} \mathrm{H}_{13} \mathrm{~F}_{3} \mathrm{O}_{2}$ \\
\hline chemical_formula_weight & 222.20 \\
\hline _space_group_crystal_system & triclinic \\
\hline space_group_IT_number & 2 \\
\hline space group name $\mathrm{H}-\mathrm{M}$ alt & 'P -1' \\
\hline
\end{tabular}


_space_group_name_Hall_'-P 1'

_space_group_symop_operation_xyz

_cell_length_a

$5.9984(18)$

_cell_length_b

$12.944(6)$

cell_length_c

$13.935(6)$

_cell_angle_alpha

$66.928(8)$

_cell_angle_beta

$80.968(10)$

_cell_angle_gamma

$83.515(9)$

_cell_volume

981.5(7)

cell_formula_units_Z

4

cell_measurement temperature

cell_measurement_reflns_used 331

_cell_measurement_theta_min 3.18

cell_measurement_theta_max 21.51

_exptl_crystal_description 'block'

_exptl_crystal_colour_colourless'

_exptl_crystal_density_diffrn 1.504

_exptl_crystal_F_000 464

_exptl_crystal_size_max 0.500

_exptl_crystal_size_mid 0.290

_exptl_crystal_size_min $\quad 0.200$

_exptl_absorpt_coefficient_mu 0.140

_shelx_estimated_absorpt_T_min 0.933

_shelx_estimated_absorpt_T_max 0.973

_exptl_absorpt_correction_type numerical

_exptl_absorpt_correction_T_min 0.68

_exptl_absorpt_correction_T_max 0.97

exptl absorpt process details SADABS

_diffrn_ambient_temperature 173(2)

_diffrn_radiation_wavelength 0.71073

_diffrn_radiation_type MoKla

_diffrn_radiation_source_sealed tube'

_diffrn_radiation_monochromator 'graphite'

_diffrn_measurement_device_type 'CCD area detector'

diffrn_measurement_method_omega scan'

diffrn_reflns_number $\quad 10748$

diffrn_reflns_av_unetI/netI 0.0834

_diffrn_reflns_av_R_equivalents 0.0555

_diffrn_reflns_limit_h_min -7

_diffrn_reflns_limit_h_max 7

_diffrn_reflns_limit_k_min -15

_diffrn_reflns_limit_k_max 14

_diffrn_reflns_limit_1_min -17

_diffrn_reflns_limit_1_max 17

_diffrn_reflns_theta_min 1.713

diffrn reflns theta max 25.998

_diffrn_reflns_theta_full 25.242

diffrn_measured_fraction_theta_max 0.999

diffrn measured fraction theta full 0.999

_diffrn_reflns_Laue_measured_fraction_max 0.999

_diffrn_reflns_Laue_measured_fraction_full 0.999

_diffrn_reflns_point_group_measured_fraction_max 0.999

_diffrn_reflns_point_group_measured_fraction_full 0.999 
_reflns_number_total

_reflns_number_gt

2002

_reflns_threshold_expression 'I $>2 \backslash \mathrm{s}(\mathrm{I}){ }^{\prime}$

Structure 29b*HCl (1850045)

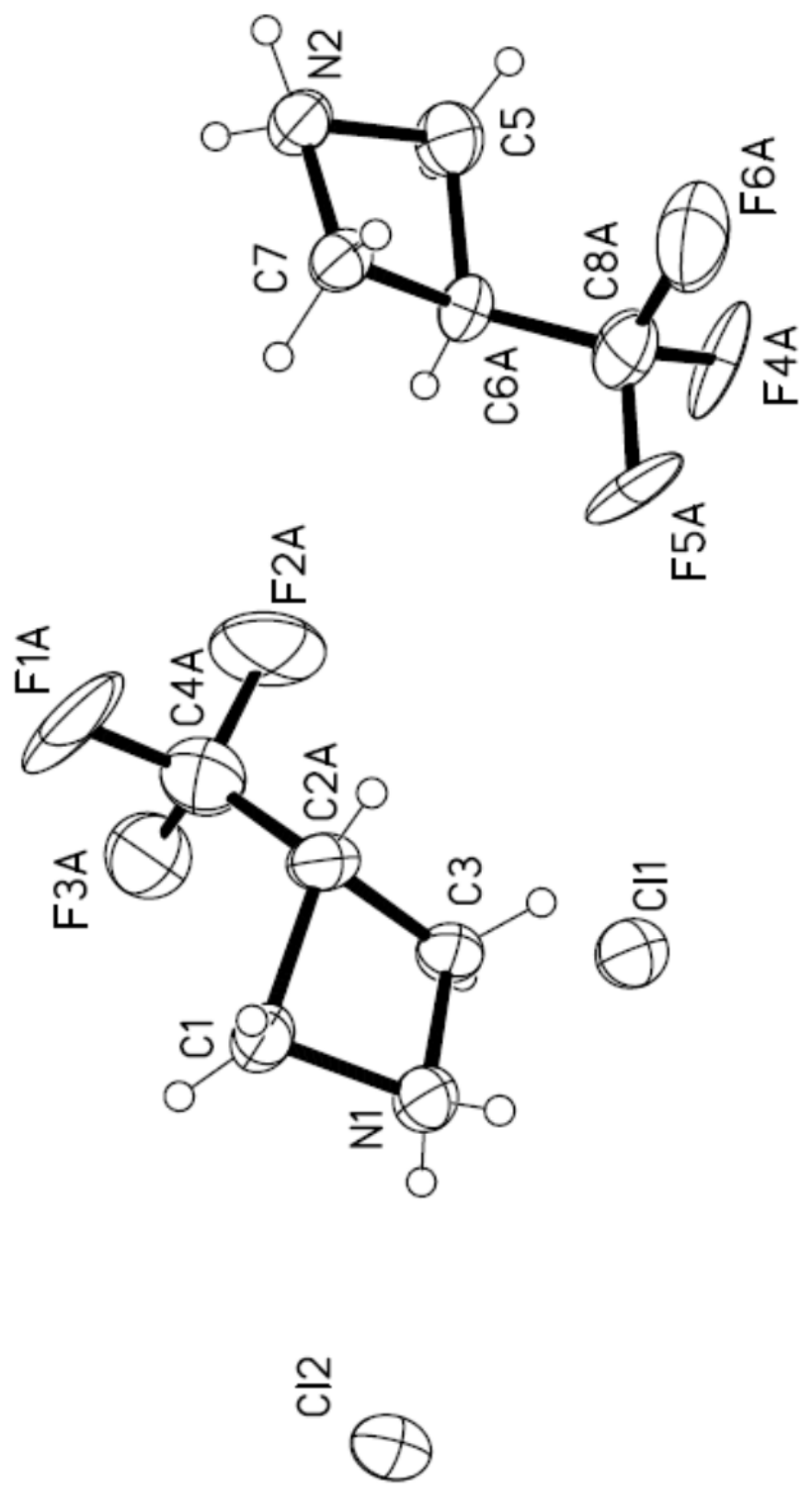

Figure S4. Structure of compound $29 \mathbf{b} * \mathbf{H C l}$ according to X-ray diffraction data. Ellipsoids are shown at $30 \%$ probability level.

\section{Crystal data and structure refinement parameters:}

_name_xr30

_audit_creation_method_SHELXL-2016/4'

_shelx_SHELXL_version_number '2016/4'

_chemical_formula_moiety $\quad \mathrm{C}_{4} \mathrm{H}_{7} \mathrm{~F}_{3} \mathrm{NCl}$

_chemical_formula_weight 161.56 


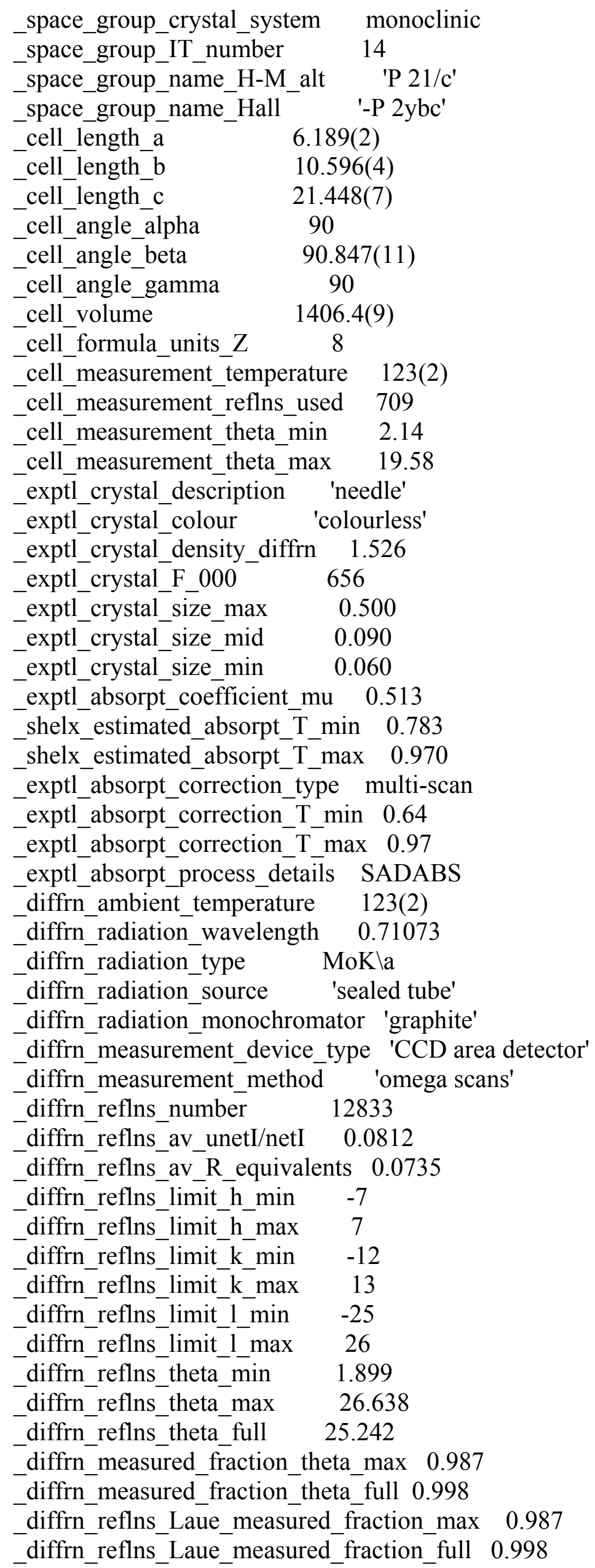


_diffrn_reflns_point_group_measured_fraction_max 0.987

_diffrn_reflns_point_group_measured_fraction_full 0.998

_reflns_number_total 2915

_reflns_number_gt 1820

_reflns_threshold_expression_I $>2 \backslash \mathrm{s}(\mathrm{I}){ }^{\prime}$

Compound 39b*HClO 4 (1850046)

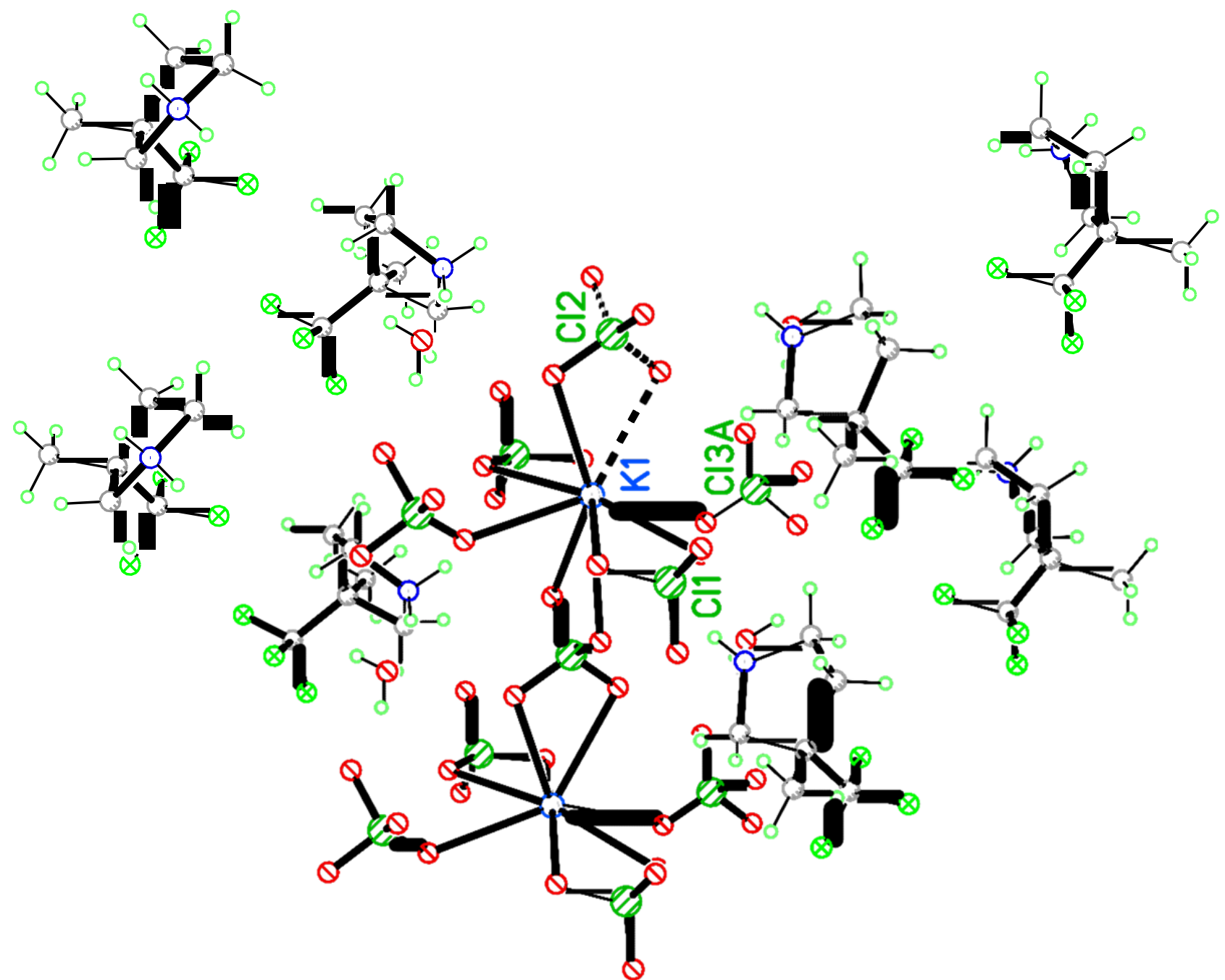

Figure S5. Structure of compound $39 \mathbf{b} * \mathbf{H C l O}_{4}$ according to X-ray diffraction data. Ellipsoids are shown at $30 \%$ probability level.

\section{Crystal data and structure refinement parameters:}

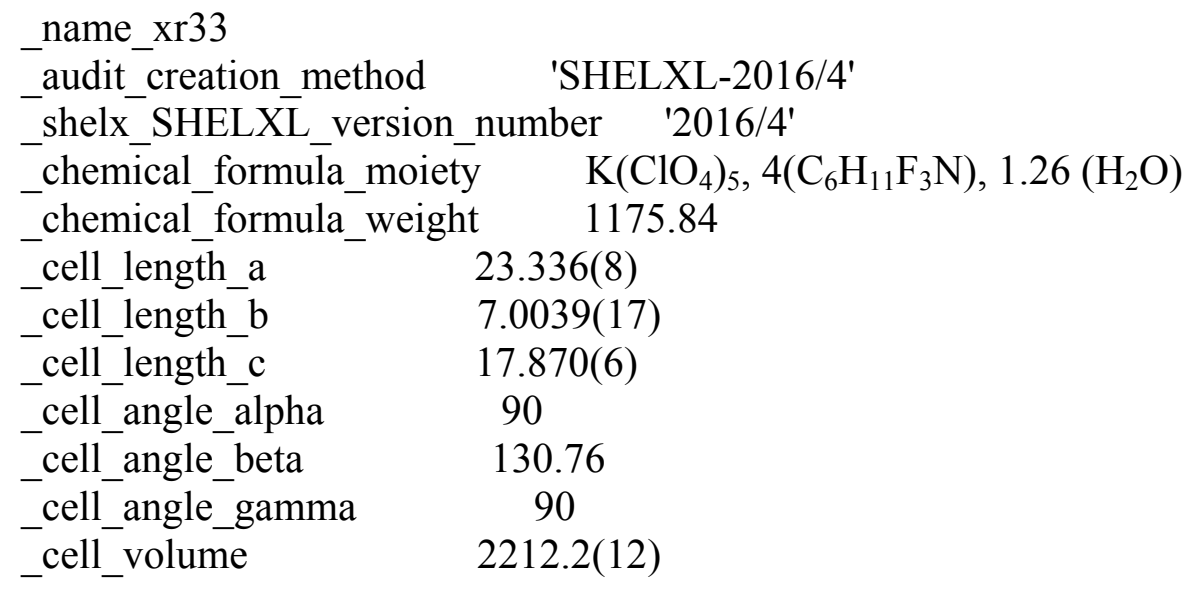


_cell_formula_units_Z

cell measurement temperature

_cell_measurement_reflns_used

_cell_measurement_theta_min

$123(2)$

cell_measurement_theta_max 19.23

566

_exptl_crystal_description needle

_exptl_crystal_colour colourless

_exptl_crystal_density_diffrn 1.765

_exptl_crystal_F_000 1201

_exptl_crystal_size_max $\quad 0.500$

_exptl_crystal_size_mid $\quad 0.100$

_exptl_crystal_size_min 0.060

_exptl_absorpt_coefficient_mu 0.554

_shelx_estimated_absorpt_T_min 0.769

_shelx_estimated_absorpt_T_max 0.967

_exptl_absorpt_correction_type numerical

_exptl_absorpt_correction_T_min 0.56

_exptl_absorpt_correction_T_max 0.97

_exptl_absorpt_process_details SADABS

_diffrn_ambient_temperature 123(2)

_diffrn_radiation_wavelength 0.71073

_diffrn_radiation_type MoKla

_diffrn_radiation_source_sealed tube'

_diffrn_radiation_monochromator 'graphite'

_diffrn_measurement_device_type 'CCD area detector'

_diffrn_measurement_method_phi and omega scans'

_diffrn_reflns_number 10320

_diffrn_reflns_av_unetI/netI 0.0892

_diffrn_reflns_av_R_equivalents 0.0695

_diffrn_reflns_limit_h_min $\quad-20$

_diffrn_reflns_limit_h_max 27

_diffrn_reflns_limit_k_min -8

_diffrn_reflns_limit_k_max 8

_diffrn_reflns_limit_1_min -21

_diffrn_reflns_limit_1_max 17

diffrn_reflns theta min $\quad 1.745$

_diffrn_reflns_theta_max 25.018

_diffrn_reflns theta_full 25.018

_diffrn_measured_fraction_theta_max 1.000

_diffrn_measured_fraction_theta_full 1.000

_diffrn_reflns_Laue_measured_fraction_max 1.000

_diffrn_reflns_Laue_measured_fraction_full 1.000

_diffrn_reflns_point_group_measured_fraction_max 0.982

_diffrn_reflns_point_group_measured_fraction_full 0.982

_reflns_number_total 3857

_reflns_number_gt 3086

_reflns_threshold_expression 'I $>2 \backslash \mathrm{s}(\mathrm{I})^{\prime}$

_reflns_Friedel_coverage 0.803

_reflns_Friedel_fraction_max 0.960

_reflns_Friedel_fraction_full 0.960 